Distribution Category: Physics-General (UC-34)

ANL-82-74

$$
\begin{aligned}
& 040-62-74 \\
& 0303005030
\end{aligned}
$$

ARGONNE NATIONAL LABORATORY

9700 South Cass Avenue

Argonne, Illinois 60439

PHYSICS DIVISION ANNUAL REVIEW

1 APRIL 1981-31 MARCH 1982

John P. Schiffer

Division Director

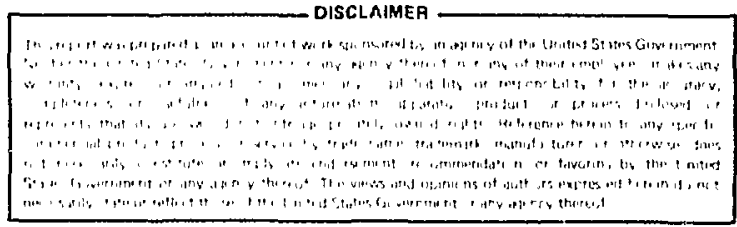

December 1982

Preceding Annual Reviews

$\begin{array}{ll}\text { ANL-79-40 } & 1978-1979 \\ \text { ANL-80-94 } & 1979-1980 \\ \text { ANL-81-79 } & 1980-1981\end{array}$


11

FOREWORD

The Physics Division Annual Review presents a broad but necessarily incomplete view of the research activity within the Division for the year ending in March 1982.

At the back of this report a complete list of publications along with the Divisional roster can be found. 
A. STUDY OF PION REACTION MECHANISMS 5

a. Study of the Pion Absorption Mechanism Through 5 the $(\pi, 2 p)$ and $(\pi, p n)$ Reactions

b. Study of the Pion Absorption Mechanism Through 6 the $\mathrm{A}(\pi . \mathrm{p}) \mathrm{X}$ Reaction at $\mathrm{T}_{\pi}=500 \mathrm{MeV}$

c. Inclusive Pion Scattering from ${ }^{4} \mathrm{He} \quad 8$

d. Surve" or Inclusive Pion Scattering Near the $\Delta_{33} \quad 10$ Reson... e

e. Inclusive Pion Charge Exchange Reactions 12

f. Inclusive Reaction Cross Sections for ${ }^{18,16} \mathrm{O}\left(\pi^{ \pm}, \pi^{0}\right) \quad 13$

g. Study of Pion Absorption in ${ }^{3} \mathrm{He}$ on and Above the 15 $(3,3)$ Resonance

h. Study of the $(\pi, \pi p)$ Reaction and Quasifree 16 Scattering in $4 \mathrm{He}$

i. Study of Low-Energy Pion Absorption in ${ }^{3} \mathrm{He} \quad 16$

j. Multiwire Proportional Chambers for LAS 17

B. NUCLEAR STRUCTURE STUDIES 18

a. Inelastic Pion scattering from ${ }^{10}{ }_{B}$ and ${ }^{11} B \quad 18$

b. Inelastic Pion Scattering from ${ }^{14} \mathrm{~N} \quad 20$

c. Excitation of High-Spin Particle-Hole States in ${ }^{28} \mathrm{Si} \quad 22$

d. Determination of Neutron Radil from Pion Scattering 23

e. Excitation of High-Spin Particle-Hole States in ${ }^{54} \mathrm{Fe} 23$

f. Study of the ${ }^{4} \mathrm{He}\left(\pi^{-}, \pi^{+}\right)$Reaction at Small Angles 24

g. Discrete States from Pion Double-Charge Exchange 24 on Heavy Nuclei

h. Dispersed Beam for LAS 25

i. Polarized Proton Scattering from ${ }^{26} \mathrm{Mg}$

j. Search for Mesonic Effects on Inelastic Proton 26 Scattering to the $10.24-\mathrm{MeV} 1^{+}$state in $48 \mathrm{Ca}$ 
C. TWO-NUCLEON PHYSICS WITH PIONS AND ELECTRONS 27

$\begin{array}{llr}\text { a. Measurement of the Angular Distribution of Tensor } & 27 \\ \text { Polarization in } \pi \text {-d Elastic Scattering } & \\ \text { b. Tensor Polarization in Electron-Deuteron Elastic } & 28 \\ \text { Scattering } & 29 \\ \text { c. Feasibility Study of Electron Scattering Experiments } & 29 \\ \text { with a Polarized Target in an Electron Storage Ring } & 30 \\ \text { d. Deuteron Tensor Polarimeter Development } & 31 \\ \text { D. NEUTRINO OSCILLATIONS } & 31 \\ \text { Neutrino Oscillations at LAMPF } & \end{array}$

II. HEAVY-ION RESEARCH AT THE TANDEM AND SUPERCONDUCTING 35 LINAC ACCELERATOR INTRODUCTION

A. HIGH ANGULAR MOMENTUM STATES IN NUCLEI

a. Transition from Collective to Aligned-Particle 37 Configuration at High Spin in 154Dy

b. High Spin Structure of ${ }^{153}$ Dy 39

c. Feeding of High-Spin Particle Yrast States in 40 151,152 Dy by Collective Cascades

d. Lifetime of Continuum States in ${ }^{154} \mathrm{Er} \quad 40$

e. The $\left(h_{11 / 2}\right)^{4}$ spectrum in the Four Valence 41

f. The $\left(\mathrm{h}_{11 / 2}\right)^{5}$ Spectrum in ${ }^{151} \mathrm{Tm}_{82}$

g. High Spin States in ${ }^{186} \mathrm{Hg} \quad 44$

h. Yrast Population Patterns in a Wide Range of Nuclei 45

B. FUSION CROSS SECTIONS 46

a. A Search for structure in the Fusion of ${ }^{16} 0+{ }^{24} \mathrm{Mg} \quad 46$

b. Influence of the Entrance Channel on the Fusion 48 Process

c. Prompt Compound Nuclear $K x$ Rays in Fusion Reactions 50 Induced by a Heavy Projectile

d. Fusion, Fission, and Deep Inelastic Reaction Cross 50 Sections for $32 \mathrm{~s}+112,116,120,124 \mathrm{Sn}$

e. Fusion of ${ }^{58} \mathrm{Ni}+114,116,118,120,122,124 \mathrm{Sn}$

C. REACTION MECHANISMS AND DISTRIBUTION OF REACTION STRENGTHS 55

a. Time-of-Flight Measurements of Evaporation Residues 55 Produced in $160+12 \mathrm{C}$ and $160+24 \mathrm{Mg}$ Reactions at $4 \leqslant E_{1 a b}(160) \leqslant 9.5 \mathrm{MeV} /$ Nucleon 
b. Observation of Final State Interaction After Heavy-Ion-Induced Fusion Reactions

c. Angular Momentum Dependence of Neutron Spectra in

d. Observation of Characteristic Gamma Rays from Quasielastic and Deep-Inelastic Fragments in the $58 \mathrm{Ni}+58 \mathrm{Ni}$ Reaction

D. RESONANT STRUCTURE IN HEAVY-ION REACTIONS

a. Search for Resonant Exchange in the Reaction $17 \mathrm{O}+48 \mathrm{Ca}$

b. Search for Massive Transfer to Molecular Resonance States in the Reaction $12 \mathrm{C}+16 \mathrm{O} \rightarrow 24 \mathrm{Mg}{ }^{*}+\alpha \rightarrow 12 \mathrm{C}$ $+{ }^{12} \mathrm{C}$ at $145 \mathrm{MeV}$

c. Elastic Scattering and Reactions of ${ }^{40} \mathrm{Ca}+{ }^{40} \mathrm{Ca}$

E. ACCELERATOR MASS SPECTROMETRY

a. Symposium on Accelerator Mass Spectrometry at the

b. Cross Section of the ${ }^{27} \mathrm{Al}(\mathrm{n}, 2 \mathrm{n}){ }^{26} \mathrm{Al}\left(\mathrm{T}_{1 / 2}=7.2\right.$ $\times 10^{5} \mathrm{yr}$ ) Reaction and Its Relevance to Displacement. Damage Studies

c. Measurement of the ${ }^{44}$ Ti Half-Life Via Tandem Accelerator Mass Spectrometry

F. SELECTED NUCLEAR SPECTROSCOPY AT THE TANDEM/LINAC

a. Laser Spectroscopy of Radioactive Atoms

b. Mass and Low-Lying States of ${ }^{106,109}$ In

c. The $\gamma$ Decay of States in ${ }^{47} \mathrm{Cr}$

G. EQUIPMENT DEVELOPMENT

a. The Split-Pole Magnetic Spectrograph in the Linac 69 Experimental Area

b. The $\gamma$-Ray Facility

c. A Plunger for Recoil Distance Lifetime Measurements

d. Superconducting Solenoid Lens Electron Spectrometer

e. Design Study of a BGO Anti-Compton Spectrometer

f. Nuclear Target Making and Development 
Page

1. WEAK INTERACTIONS

a. Parity Violation in the 5.1-MeV $J=2$ Doublet of ${ }^{10}{ }_{B} \quad 74$

b. The Beta Decay Rate of ${ }^{16} \mathrm{~N}\left(0^{-}, 120 \mathrm{keV}\right)$ : Meson 76 Exchange Currents and the Induced Pseudoscalar Coupling Constant

c. Beta Spectrum Measurements 79

d. Beta Decay Branching Ratios of ${ }^{16} \mathrm{~N}\left(2^{-}\right) \quad 79$

e. Beta Decay of ${ }^{16} \mathrm{C} \quad 80$

f. A Search for Axions from Nuclear Decays 80

2. REACTIONS USING LIGHT NUCLEI 81

a. Uncertainties in the Calculation of the Solar 81 Neutrino Flux

b. The ${ }^{7} \mathrm{Li}(\mathrm{d}, \mathrm{p})^{8} \mathrm{Li}$ Reaction Cross Section Near $0.78 \mathrm{MeV} 83$

c. The ${ }^{7} \mathrm{Li}(\mathrm{d}, \mathrm{p})^{8} \mathrm{Li}$ Reaction 85

d. The ${ }^{7} \mathrm{Be}(\mathrm{p}, \gamma)^{8} \mathrm{Be}$ Reaction 85

e. Study of Nonresonant Capture in the ${ }^{27} \mathrm{Al}(\mathrm{p}, \gamma)$ and 87 $19 \mathrm{~F}(\mathrm{p}, \gamma)$ Reactions at Low Energy

3. SEARCHES FOR EXOTIC PARTICLES

A Cryugenic Search for $+1 / 3$ e Stable Charges 88

IV. NEUTRON AND PHOTONUCLEAR PHYSICS 89

$\begin{array}{ll}\text { INTRODUCTION } & 89\end{array}$

A. NEUTRON RESEARCH 9

Measurement of the Electric Dipole Moment of the 90 Neutron

B. PHOTONUCLEAR PHYSICS

a. Photojisintegration of the Deuteron 92

b. Isospin Splitting of the Giant Dipole Resonance 94

V. THEORETICAL NUCLEAR PHYSICS 95 $\begin{array}{ll}\text { INTRODUCTION } & 95\end{array}$

A. HEAVY-ION DIRECT-REACTION THEORY 96
a. Ptolemy
96
b. Optical Potentials for Coupled-Channels Calculations
97
c. Sensitive Radius 
B. NUCLEAR SHELL THEORY AND NUCLEAR STRUCTURE 99

a. Seniority Isomers Near $Z=64 \quad 99$

b. High Spin States in ${ }^{94} \mathrm{Ru}$ and ${ }^{95} \mathrm{Rh} \quad 99$

c. Electron Scattering to $10^{-}$States in ${ }^{90} \mathrm{Zr} \quad 100$

d. Electron Scattering on ${ }^{6} \mathrm{Li} \quad 101$

e. Effects of the $\Delta$ Resonance on M1 Properties of ${ }^{17} \mathrm{O} \quad 101$

f. Strong Ml Decays in ${ }^{12} \mathrm{C} \quad 101$

g. Distribution of Gamow-Teller Strength 102

h. Analysis of Inelastic Scattering of Pions 102

C. NUCLEAR FORCES AND SUBNUCLEON DEGREES OF FREEDOM 104

a Nuclear Saturation from Two-Nucleon Potentials 105

b. Three-Body Forces in Light Nuclei and Nuclear Matter 107

c. Isobar Degrees of Freedom and Three-Body Forces in 108 Nuclear and Neutron Matter

d. Nucleon-Nucleon Potentials with Isobars 109

e. Phenomenological Hamiltonian for Pions, Nucleons 110 and $\triangle$ Isobars

f. Relativistic Quantum Mechanics of Particles with 111 Direct Interactions

g. Strong Coupling Approximations to Chiral Bag Models 112

D. INTERMEDIATE ENERGY PHYSICS 114

a. A Microscopic Study of $\Delta$-Nucleus Potentials 114

b. A Study of the Inclusive Reaction ${ }^{4} \mathrm{He}(\pi, \mathrm{p}) \quad 114$

c. Scattering and Absorption of Pion by the $A=3$ Nuclei 115

d. Intermediate Energy Electron Scattering 115

e. Spin and Isospin Effects in Elastic p- ${ }^{4} \mathrm{He} \quad 116$ Scattering Near $1 \mathrm{GeV}$

f. Elastic and Inclusive Proton-Nucleus Scattering 116 Above $500 \mathrm{MeV}$

E. MICROSCOPIC CALCULATIONS OF HIGH-ENERGY COLLISIONS OF 118 HEAVY IONS

a. Classical-Equations-of-Motion Calculation of 118 Multiplicities for High-Energy Collisions of $20 \mathrm{Ne}+20 \mathrm{Ne}$

b. Interaction and Collective Effects in Classical- $\quad 118$ Equations-of-Motion Calculations

c. Improvements and Extensions of the CEOM Calculations 119

d. Theory of High-Energy Heavy-Ion Collisions 119 
F. OTHER THEORETICAL PHYSICS 120

a. Aharonov-Bohm Effect 120

b. Coupled Channel Formalism for Rearrangement Reactions 120

c. Applicability of the Potential Model of Colored 121 Quarks for Nucleon Interactions

d. Model Independent Analysis of Experimental Baryon 121 Magnetic Moments

VI. THE SUPERCONDUCTING LINAC 123

INTRODUCTION 123

A. PROTOTYPE HEAVY-ION SUPERCONDUCTING LINAC 124

1. STATUS OF THE BOOSTER 124

2. NEAR-TERM PLANS 124

B. INVESTIGATIONS OF SUPERCONDUCTING LINAC TECHNOLOGY 125

1. RECENT ACCOMPLISHMENTS 125

a. Superconducting Resonators 125

b. Time-of-Flight Technology 126

c. Superconducting Analyzing Magnet 128

2. NEAR-TERM PLANS 129

$\begin{array}{lr}\text { C. THE ATLAS PROJECT } & 130\end{array}$

VII. ACCELERATOR OPERATIONS 137

$\begin{array}{ll}\text { INTRODUCTION } & 137\end{array}$

A. OPERATION OF THE TANDEM-LINAC ACCELERATOR 138

1. OPERATION OF THE ACCELERATOR 138

2. STATUS OF THE SUPERCONDUCTING LINAC 141

3. UPGRADING OF THE LINAC 142

a. Resonator Fabrication 142

b. Computer Control System 142

c. Liquid-Nitrogen Distribution 142

d. Slow-Tuner Pressure Controller 143

e. Liquid-Helium Control System 143

f. Heavy-Ion Beam Switch 143

8. Energy Measurement System 144

4. UPGRADING OF ThE TANDEM 144

a. Stripping Foils 144 
b. Terminal-Control System 145

c. Charging System 145

d. On-Line Ion Source 145

e. Beam-Line and Accelerator Control 145

5. ION-SOURCE DEVELOPMENT 145

a. Ion-Source Test Facility 146

b. New Design for the Inverted Sputter Source 146

c. West Injector for the Tandem 146

d. The Aarhus and SNICS Sources 146

6. NEAR-TERM PLANS 147

a. Accelerator Operation 147

b. Booster Improvements 147

c. Tandem Improvements 147

d. Ion-Source System 147

7. ASSISTANCE TO OUTSIDE USERS OF THE TANDEM-LINAC 148 ACCELERATOR

a. Experiments Involving Outside Users 148

b. Outside Users and Institutional Affiliations 150

c. Summaries of Major User Programs 152

B. OPERATION OF THE DYNAMITRON FACILITY 155

1 OPERATIONAL EXPERIENCE 155

2. UNIVERSITY USE OF THE DYNAMITRON 157

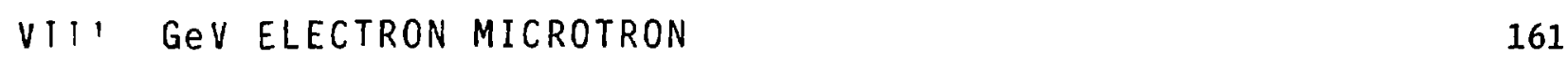

$\begin{array}{ll}\text { INTRODUCTION } & 161\end{array}$

a. Status of Microtron Sector Magnet Studies 164

b. Future Plans 164

ATOMIC AND MOLECULAR PHYSICS RESEARCH 167

$\begin{array}{ll}\text { INTRODUCTION } & 167\end{array}$

1X. DISSOCIATION AND OTHER INTERACTIONS OF ENERGETIC MOLECULAR IONS IN SOLID AND GASEOUS TARGETS 
a. Molecular-Ion Stopping Power Measurements 170

b. Channeling of Molecular-Ion Beams $\quad 170$

c. Computer Simulations of Foil-Induced Dissociations 173

d. Electron Spectroscopy of Fast Molecular Ions 173

e. Equipment Development 173

f . Contribution of Field-Ionized Rydberg Atoms to 174 Convoy Electron Spectra

$\begin{array}{lll}X . & \text { ELECTRON SPECTROSCOPY WITH FAST ATOMIC AND } & 179\end{array}$ MOLECULAR-ION BEAMS

$\begin{array}{ll}\text { INTRODUCTION } & 179\end{array}$

Auger Emission from Fragments Arising from Coulomb- 180 Exploding Molecular-Ion Beams

XI. BEAM-FOIL RESEARCH AND COLLISION UYNAMICS OF HEAVY 185 IONS

$\begin{array}{ll}\text { INTRODUCTION } & 185\end{array}$

a. Coherent Excitation of Hydrogen by a Thin Carbon Foil 186

b. Molecular Effects on Lyman- $\alpha$ Emission in Beam-Foil 186 Spectroscopy

c. Orientation and Alignment Parameters of Beam-Foil- 188 Excited HeI

d. Quantum-Beat Studies of the ${ }^{3}$ He Hyperfine Structure 190

e. Lamb Shift and Fine Structure of $n=2$ in Helium- 190 like Chlorine, Sulfur, and Silicon

f. Position Sensitive Detector for UV Spectroscopy 192

g. Doubly-Excited States in Lithium 192

h. The Doubly-Excited Singlet States in Helium 193

i. Spectra of High Spin States in Light Elements 193

j. Measurement of the Transition Probability of 105 Singlet-Triplet Intercombination Lines in Neon

k. Periodic Wavelength Dispersion in a Scanning UV 196 Monochromator

XII. PHOTOIONIZATION-PHOTOELECTRON RESEARCH 197 $\begin{array}{ll}\text { INTRODUCTION } & 197\end{array}$

a. Photoelectron Spectra of Open-Shell Atoms, with 200 Emphasis on Tellurium 
b. Photoelectron Spectra of Lanthanide Trihalides

Page

c. Photoionization Mass Spectrometry of $\mathrm{NH}_{2} \mathrm{OH}$ : Heats

d, Photoionization Mass Spectrometric Study of $\mathrm{CH}_{3} \mathrm{SH}$, $\mathrm{CD}_{3} \mathrm{SH}$, and $\mathrm{CH}_{3} \mathrm{SD}$

XIII. HIGH-RESOLUTION LASER-rf SPECTROSCOPY WITH ATOMIC AND MOLECULAR BEAMS

INTRODUCTION

a. Hfs of the Molecular Ground State $\left(\mathrm{x}^{2} \Sigma\right)$ of $\mathrm{CaCl}$

b. New Method for Resolution of Unresolved hfs Components in Optical Spectra: Hfs of the Excited $\mathrm{B}^{2} \Sigma$ state of $\mathrm{CaCl}$

c. Hfs of the Excited $A^{2} \pi$ state of $\mathrm{CaCl}$

d. Spin-Rotational and Hyperfine Structure of the $\mathrm{v}=1, \mathrm{X}^{2} \Sigma$ state of $\mathrm{CaBr}$

XIV. THEORETICAL ATOMIC PHYSICS

a. Radiative Transitions Between Quintet States of

b. Nonrelativistic Total Energy for the (1s $3 d)^{1} D$

c. Intershell Correlation Corrections to the Energy Levels of Few-Electron Ions

d. Correlation and Relativistic Effects in Spin-Orbit Splittings

e. Theoretical Photoionization Parameters for the

f. Photoionization of Group-IIB Elements 


\title{
NUCLEAR PHYSICS RESEARCH
}

\author{
INTRODUCTION
}

\begin{abstract}
The research program in nuclear physics in the Argonne Physics Division contributes to most of the major questions in this scientific discipline. The development of the superconducting rf linac technology has led to the ATLAS project now under construction, and is the major focus of the effort in heavy-ion research. The investigation of pion interactions in nuclei using the LAMPF facility helps delineate the dominant aspects of pion propagation and interactions in nuclei. Experiments on the weak interaction in nuclei and on other fundamental symmetries are being carried out with a number of tools. The theoretical effort in nuclear siructure, nuclear matter and dynamics, and reaction theory is closely coupled to many aspects of the experimental programs.
\end{abstract}

The Physics Division operates a superconducting linac booster: injected by a tandem accelerator, and a 4-MV Dynamitron. The linac is used almost entirely for nuclear research by Argonne staff and university users. Less than $25 \%$ of the Dynamitron is used for nuclear research. A small program in photonuclear research is carried out at the Chemistry Division's electroin linac, and occasionally elsewhere. The medium-energy program is centered at the Los Alamos Meson Physics Facility, with occasional experiments elsewhere.

\section{$\underline{\text { Highlights }}$}

In medium-energy pion physics, considerable progress has been made in understanding the propagation and absorption of pions (deltas) in the nuclear medium. Qualitatively new results were obtrined for the $\pi+2 \mathrm{~N} \rightarrow 2 \mathrm{~N}$ reaction.

An experiment to study neutrino oscillations is being planned at LAMPF with substantial involvement from Argonne.

A major effort is being devoted to the scientific and technical considerations involved in proposing to build a multi-GeV C.W. electron accelerator: GEM at Argonne.

In heavy-ion physics, the superconducting linac booster is being used with increasing scientific profit. A split-pole spectrograph has become operational, the large scattering chamber is better instrumented and a new beam line has been installed. Results on high-spin states and on various systematic effects in heavy-ion reactions are increasing. The outside user involvement is flourishing.

Construction of the ATLAS facility began in FY 1982 and all progress has been on schedule. 
The importance of the time component of the weak axial-vector current has been studied through the $\beta$ decay of $16 \mathrm{~N}$.

A precision measurement is under way of the ${ }^{7} \mathrm{Be}(\mathrm{p}, \gamma)$ cross section, one of the key components in the solar neutrino anomaly. Preliminary results indicate that some fraction of the anomaly may disappear with the new measurement.

In nuclear theory, during the past year, the coupled-channel code for treating heavy-ion inelastic scattering was completed and application to particular experiments began. Nuclear structure theory was applied to interpret decays of high-spin states and inelastic pion scattering. Results of particular interest were obtained in the nuclear force program where the inclusion of 3-body forces led to simultaneous inprovement in the binding of ${ }^{3} \mathrm{He}$ and ${ }^{4} \mathrm{He}$ and saturation $=\mathrm{f}$ nuclear matter. 


\title{
I. MEDIUM-ENERGY PHYSICS
}

\author{
INTRODUCTION
}

The purpose of the medium-energy physics program is to explore the nature of nuclear matter by studying the fundamental particle reactions which mediate the interactions between the nuclear constituents. These basic reactions and the manner in which they are modified in the interior of nuclei can reveal important information on the character of nuclear forces. They can also serve as unique probes of nuclear structure because of the special nuclear degrees of freedom which they excite. Pion reactions receive major emphasis because the pion is generally accepted to be the basic quantum of the nuclear force. In simple nuclel such as the deuteron and the helium nucleus these reactions provide a benchmark for testing computational terhniques for handling the basic particle coupling in a multi-body nuclear system. In heavy nuclei they provide a rigorous test of phenomenological models, such as the isobar-hole doorway theory, which have been developed for interactions with complex nuclei. Electromagnetic interactions are an important component of the program because of their coupling to pionic degrees of freedom in the nucleus and because of their promise in studying the short range properties of nuclear Forces. Weak interactions are also studied, their nature being probed in a search for neutrino oscillations.

Present research trends indicate a clear national need for a high-energy continuous beam electron accelerator to pursue electromagnetic studies of the nucleus. Such a facility will address many of the questions which lie at the interface of elementary particle reactions and nuclear physics. Argonne is paricipating in a national program to develop the technology necessary to construct such an accelerator by developing a conceptual design for a GeV C.W. microtron and carrying out the research and development necessary to establish its scientific feasibility. A major effort is devoted to a prototype study of the sector magnet required for multi-sided microtron designs.

A new aspect of the ANL medium-energy program has come into existence over the past year. The arrival of new staff plus a redirection of effort from the low-energy weak interactions program has provided the personnel for the involvement in a large collaboration searching for neutrino oscillations. Neutrino oscillations are expected in gauge theorles that go beyond unlfying fust the weak and electromagnetic fields. The collaboration has an approved experiment (E-645) at LAMPF and is now in the process of detalled design of the large, fine-grained detectors and shields necessary to carry out these seirches.

During 1980-81 the medium energy research in the Argonne Physics Division has been especially fruitful. Varlous pion beams of the Los Alamos Mescn Physics Facility (LAMPF) have been utilized in the Argonne program to execute a comprehensive study of the general features of pion- 
nucleus reactions and in a series of high resolution studies of topical problems in nuclear structure possible because of the unique properties of the pion. One of the major tasks is to explore the dominant features of pion absorption and scattering from nuclel. We have improved our understanding of pion absorption by studying the 2 nucleon coincidence spectra, explored the role of pions in electromagnetic processes and obtalned new information on the partition of total cross sections between inelastic scattering and absorption. Nuclear structure studies investigated the multipole decomposition of inelastic transitions in light nuclei and searched for the tetra-neutron. The studies of the two nucleon system provided additional unexpected results, when the polarization measurements were found to be in disagreement with all current theoretical calculations.

The competition between various reaction mechanisms involved in plon propagation through nuclear matter continues to be an e:citing area of study. While many of the experiments focus upon "inclusive" spectra of particles that result from absorption or scattering, increasing effort is being placed on coincidence experiments where specific reaction mechanisios can be selected. This program is greatly facilitated by the large solid angle, dynamic range, and versat1lity of a Large Acceptance Spectrometer (LAS) constructed for this serles of measurement;.

Studies of elastic and inelastic pion scattering are primarily directed toward understanding the relationship between pion nuclear reactions and nuclear structure. While high-resolution experiments continue to utilize the EPICS system, moderate resolution experiments are being performed with LAS. Nuclear structure studies involving highresolution scatterine, and high excitation energies are analyzed in terms of microscopic descriptions of states.

Knowledge of the behavior of few-nucleon systems and their interaction with pions is a prerequisite for understanding pion interactions in complex nuclei. Measurements of deuteron polarization from both pion and electron scattering provide a unique method for testing various models that attempt to describe the fundamental interactions. The analysis of the latest measurements of deuteron tensor polarization in $\pi-d$ scattering indicates the need for consideration of dibaryon resonances or other hitherto neglected mechanisms. An increasing fraction of the program is devoted to experiments using the electron beams of the MIT/Bates accelerator, and a novel concept involving an electron storage ring is being evaluated. 


\section{A. STUDY OF PION REACTION MECHANISMS}

Even though it is crucial to a description of nuclei, our understanding of pion propagation in complex nuclei is still quite incomplete. The details of the processes involved in the various absorption mechanisms are of Importance, as is the competition between scattering and absorption. It is found that direct $(\pi, p n)$ reactions on the are strongly suppressed relative to $\left(\pi^{+}, 2 \mathrm{p}\right)$ reactions. This is in accord with a description of pion absorption in which an intermediate $\Delta$-nucleon state of well-defined angular momentum and parity is formed prior to the decay into two nucleons. Inclusive reactions involving both inelastic scattering and charge exchange demonstrate the intimate relationship between the reactive components of inelastic scattering and absorption. Further progress toward disentangling quasi-free scattering from multistep inelastic scattering, establishing the Iimits of validity of the impulse approximation and the isobar-hole model, and elucidating nuclear structure effects upon the macroscopic reaction cross saction has been made.

\section{a. Study of the Pion Absorption Mechanism Through the $(\pi, 2 p)$ and $(\pi, p n)$}

Reactions

D. Ashery, R. J. Holt, H. E. Jackson, J. P. Schiffer, J. R. Specht,

K. E. Stephenson, R. D. McKeown, ${ }^{*}$ J. Ungar, ${ }^{*}$ R. E. Segel, ${ }^{\dagger}$ and

P. Zupranski ${ }^{\dagger}$

We have carried out a colncidence measurement of the $\mathrm{He}\left(\pi^{+}, 2 \mathrm{p}\right)$ and $\left(\pi^{-}, \mathrm{pn}\right)$ reactions on ${ }^{3} \mathrm{Be}$ and ${ }^{4} \mathrm{Be}$ at $165 \mathrm{MeV}$. The ratio of the cross sections for these reactions is a sensitive test of the reaction mechanism. By using isospin Clebsch-Gordan coefficlents for calculating this ratio and assuming $\Delta$ dominance of the process, the result is $\sigma\left(\pi^{-}, \mathrm{pn}\right) / \sigma\left(\pi^{+}, 2 \mathrm{p}\right)=0.13$ (for ${ }^{3} \mathrm{He}$ ) and 0.08 (for ${ }^{4} \mathrm{He}$ ). However, these results will be different if the reaction mechanism includes formation of a $\Delta N$ intermediate state with a well-defined angular momentum and parity before the $\Delta N+N N$ transition. In particular, if this intermediate state will have an even relative angular momentum ( $L_{\Delta N}$ $=0,2)$, absorption on a $T=1, J=0$ nucleon pair will be forbidden by angular momentum and parity conservation. This condition will lead to a strong suppression of the $(\pi, \mathrm{pn})$ reaction in hellum. The measurements were done in the $\mathrm{P}^{3}$ channel at LAMP by detecting protons with the LAS and the coincident

\footnotetext{
${ }^{*}$ California Institute of Technology, Pasadena, California.

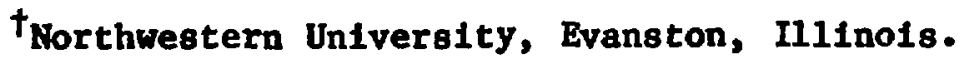


protons or neutrons with two $15-$ by $15-$ by $35-\mathrm{cm}$ plastic scintillators. The scintillators were positioned so that one of them vas at the angle where the direct $(\pi, 2 \mathrm{~N})$ nucleon-nucleon angular correlation is peaked and the second at the tail of the correlation. The experimental data allow for the separation of the direct $(\pi, 2 \mathrm{~N})$ absorption from the process where the pion energy is shared with more nucleons. Analysis of the data, shown in Fig. I-1, Indicates that the direct $(\pi, p n)$ reaction is strongly suppressed by about an order of magnitude with respect to the predictions mentioned above.

b. Study of the P1on Absorption Mechan1sm Through the A( $\pi, P) X$ Reaction at $T_{-}=500 \mathrm{MeV}$

D. Ashery, R. J. Holt, H. E. Jackson, R. D. McKeown, * J. P. Schiffer, R. E. Segel, ${ }^{\dagger}$ K. E. Stephenson, and J. R. Specht

We have measured Inclusive proton spectra from $(\pi, p)$ reactions on targets of ${ }^{3} \mathrm{He},{ }^{4} \mathrm{He}, \mathrm{C}, \mathrm{NI}$ and $\mathrm{Ta}$ at $81 \mathrm{x}$ angles for a plon kinetic energy of $T_{\pi}=500 \mathrm{MeV}$. The Large-Acceptance Spectrometer (LAS) was used to detect the protons. The spectrometer was originally designed to detect pions up to $\sim 500 \mathrm{MeV} / \mathrm{c}$, but relatively minor modifications allowed detection of protons up to $\sim 1400 \mathrm{MeV} / \mathrm{c}$. This work complements the results of an earlier experiment, Exp. 350 , In which $(\pi, p)$ cross sections were measured in the region of the $\Delta$ resonance. The results of Exp. 350 Imply that pion absorption proceeds through a relatively large number of nucleons. Without additional information off-resonance, however, it is difficult to disentangle unambiguously which features of the proton spectra originate from the pion absorption vertex and which from subsequent rescattering of the emerging protons. If the main features of the proton spectra in Exp. 350 are due to the absorption vertex, we expect qualitatively different results at $T_{\pi}=500 \mathrm{MeV}$, where the $\pi$ iv scattering cross section is roughly a factor of 10 smaller than at resonance. Analysis of the data 18 in progress.

\footnotetext{
*California Institute of Technology, Pasadena, California.

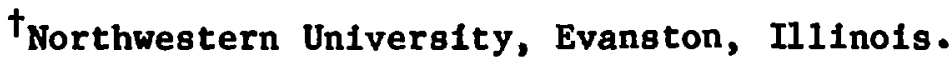




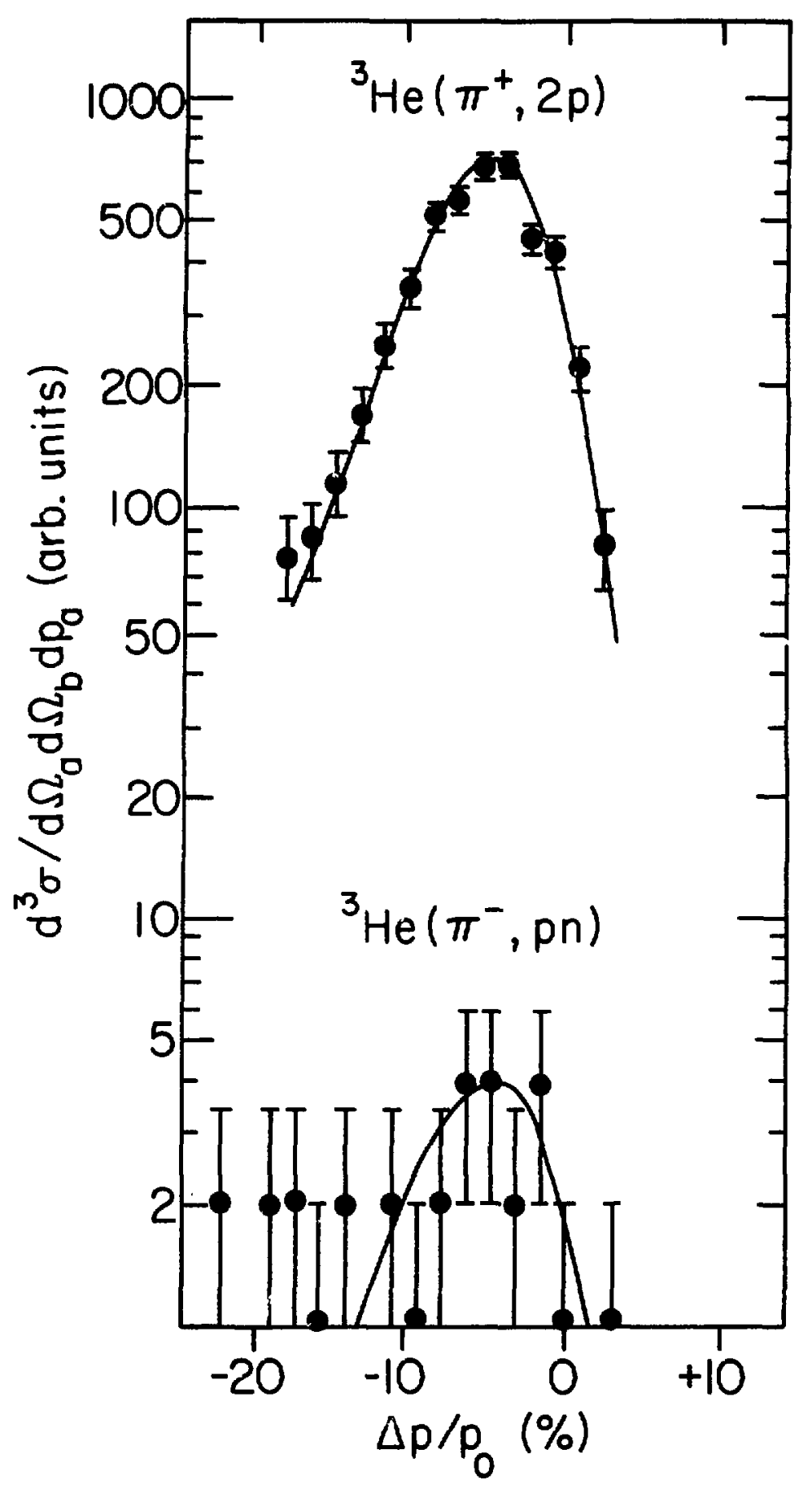

Fig. I-1. Momentum spectra of protons detected with the magnet at $55^{\circ}$ with $165-\mathrm{MeV}$ pions incident on a ${ }^{3} \mathrm{He}$ target. The upper curve was taken with $\pi^{+}$and protons in coincidence in a scintillation arm at $-100^{\circ}$; while the lower curve $1 s$ with $\pi^{-}$and neutrons in coincidence. The two sets of data are presented with the correct relative normalization. 
c. Inclusive Pion Scatcering from ${ }^{4} \mathrm{He}$

E. P. Colton, * D. F. Geesaman, R. J. Holt, H. E. Jackson, S. Levenson, ${ }^{\dagger}$

J. P. Schlffer, J. R. Specht, K. E. Stephenson, B. Zeldman, R. E.

Segel, P. Gram, and C. Goulding

We have measisred inclusive pion scattering from ${ }^{4} \mathrm{He}$ at incident $\pi^{+}$ energles of 100,160 and $220 \mathrm{MeV} .1$ The experiment was performed using the LAS spectrometer at ios Alamos and a liquid ${ }^{4} \mathrm{He}$ target. Pion-energy spectra were accumulated at 5 angles from 30 to $146^{\circ}$. The results at the forward angles and the results at $100 \mathrm{MeV}$ are somewhat different trom those observed on ${ }^{2} \mathrm{C}$, ${ }^{58} \mathrm{Ni}$, and ${ }^{208} \mathrm{~Pb}$ as a consequence of the tight binding of the ${ }^{4}$ He nucleus. Based on elastic scattering and total cross sections from the literature, our results, 1llustrated In FIg. I-2, Imply that the rue absorption cross section Is a particularly large fraction of the total reaction cross section $(70 \pm$ 20\%) at $100 \mathrm{MeV}$ while it 18 a relatively small part of the total cross section at 160 and $220 \mathrm{MeV}(\sim 20-30 \%)$. The elastic cross section remains a constant fraction of the toral cross section over the same energy range. These results illustrate the importance of competition between the reactive components, Inelastic scattering and absorption, of the pion-nucleus interaction.

\footnotetext{
*Accelerator Research Factlities Division, ANL.

thesis student, University of Chicago, Chicago, Illinols.

\# Northwestern University, Evanston, Illinols.

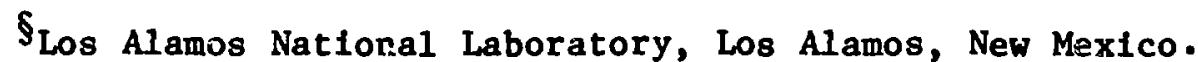

Il Florida A \& M University, Tallahassee, Florida.

$1_{S}$. M. Levenson, D. F. Geesaman, E. P. Culton, R. J. Holt, H. E. Jackson, J. P. Schiffer, J. R. Specht, K. E. Stephenson, B. Zeldman, R. E. Segel, P. A. M. Gram, and C. A. Goulding, Phys. Rev. Lett. 47, 479 (1981).
} 


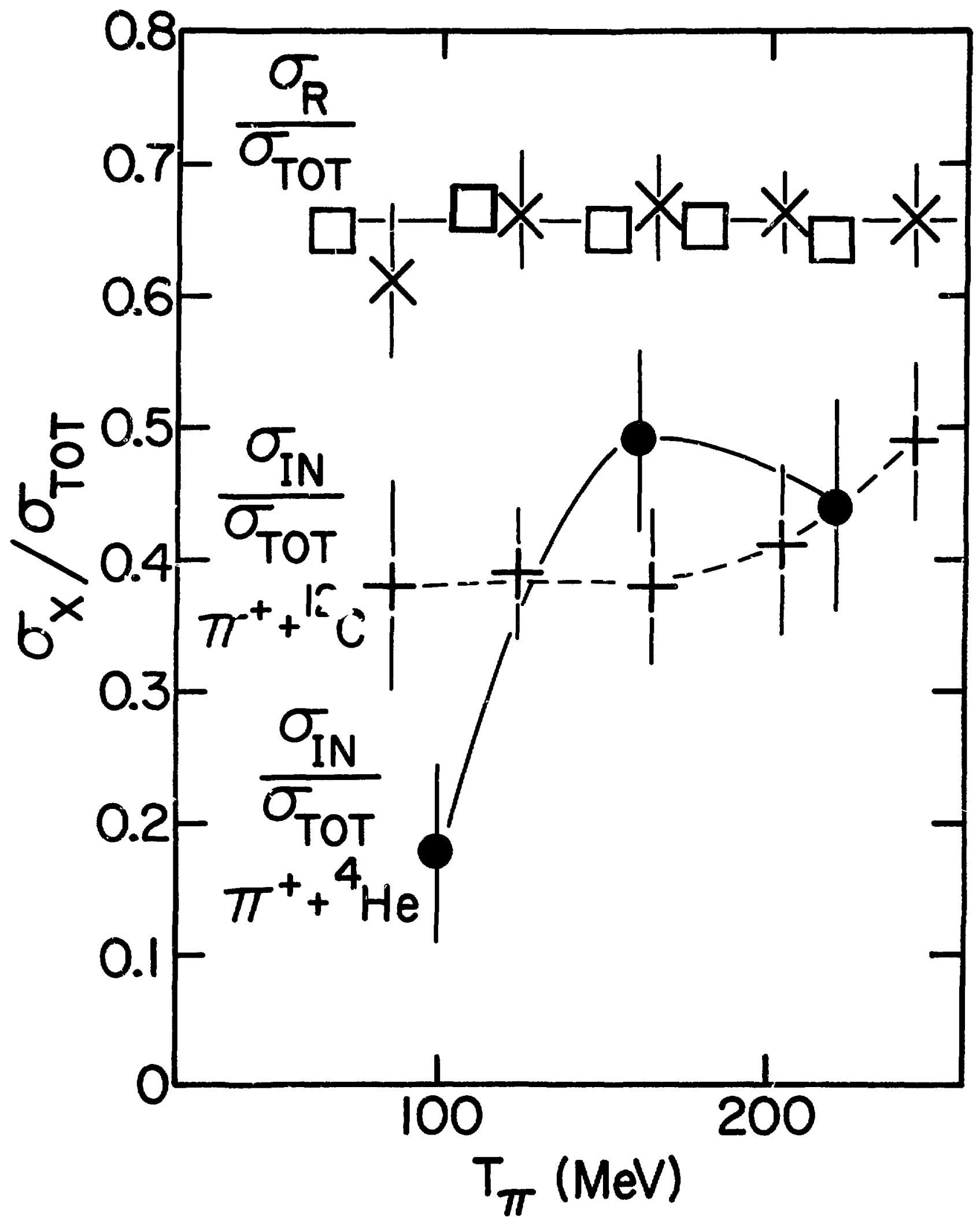

F1g. I-2. The ratio of $\sigma_{I N}$ and $\sigma_{R}$ to $\sigma_{T O T}$ for ${ }^{4} \mathrm{He}$ (circles and squares, respectively). Experimental $\pi^{-}$data and charge independence were used to obtain $\sigma_{R} / \sigma_{T O T}$. The same ratios for $\pi^{+}+12 \mathrm{C}$ are shown by the pluses and $x^{\prime} s$, respectively. The lines are drawn to guide the eye. 


\section{d. Survey of Inclusive P1on Scattering Near the $\Delta_{33}$ Resonance}

E. P. Colton, ${ }^{\star}$ D. F. Geesaman, R. J. Holt, H. E. Jackson, S. Levenson, ${ }^{\dagger}$

J. P. Schiffer, J. R. Specht, K. E. Stephenson, B. Zeldman, R. E. Segel, ${ }^{\ddagger}$

P. Gram, and C. Goulding

A comprehensive series of measurements of inclusive pion scattering from ${ }^{12} \mathrm{C},{ }^{58} \mathrm{NI}$, and ${ }^{208} \mathrm{~Pb}$ with the LAS spectrometer have been completed. PIon energy spectra were measured at 7 angles from $30^{\circ}$ to $146^{\circ}$ at $\pi^{+}$Incident energles of 100,160, and $220 \mathrm{MeV}$. Data were also obtained on an $\mathrm{H}_{2} \mathrm{O}$ target at $100 \mathrm{MeV}$, on ${ }^{12} \mathrm{C}$ at $300 \mathrm{MeV}$ and on $\pi^{-}$scattering from ${ }^{12} \mathrm{C}$ and ${ }^{208} \mathrm{~Pb}$ at $160 \mathrm{MeV}$.

The plon-energy spectra, shown for 220-MeV incldent energy in Fig. I-3, are dominated by a large peak at an energy near that corresponding to plons scattering from a free nucleon. This indicates the importance of the quasifree reaction mechanism even in the presence of strong pion absorption. Indeed, the spectra look rather similar for each target and the angular distributio.s follow the free $\pi$-nucleon angular distribution at back angles. At forward angles however, the significant yleld of low-energy plons cannot arise from the quasifree mechanism and yleld in the quasifree peak is lower than that expected from a free-nucleon-like angular distribution. These observations signal the Importance of higher-order processes and Pauli blocking effects in the reaction mechanism.

Total Inelastic scattering cross sections have been obtained for each target by integrating the energy and angular distributions. Inelastic scattering accounts for between $30 \%$ and $60 \%$ of the total reaction cross sections for these nuclei. A manuscript reporting the results of this work is In preparation.

\footnotetext{
*Accelerator Research Facilities Division, ANL.

${ }^{+}$Thesis student, University of Chicago, Chicago, Illinois.

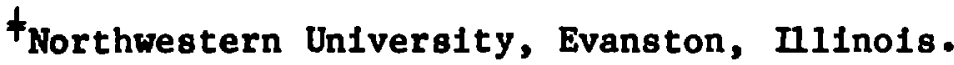

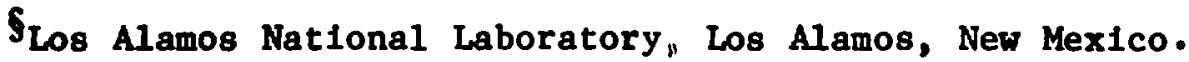

II Florida A \& M University, Tallahassee, Florida.
} 


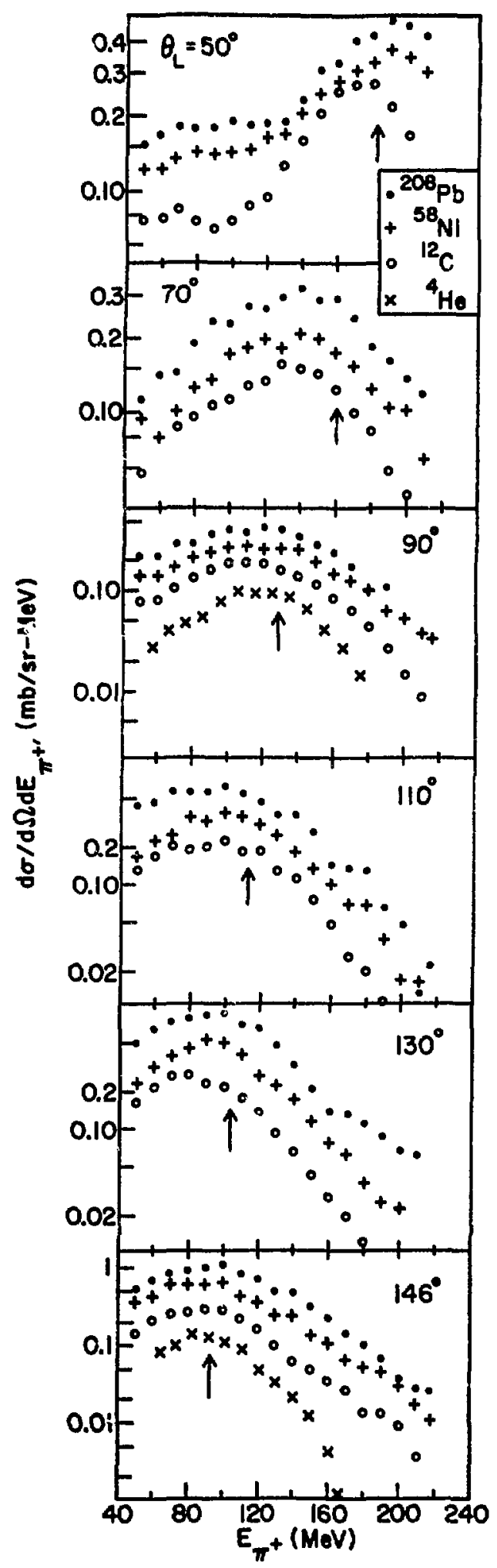

Fig. I-3. The inelastic spectra at $T_{\pi}=220 \mathrm{MeV}$ are shown. The elastic peak and low-lying exc1ted states have been suppressed in this figure. Arrows indicate the energies corresponding to free $\pi$-nucleon kinematics. 
e. Inclusive Pion Charge Exchange Reactions

A. Airel1, ${ }^{\star}$ D. Ashery, H. W. Bauer, ${ }^{\dagger}$ M. D. Cooper ${ }^{\dagger} \mathrm{J}$. Conuzz1, ${ }^{\ddagger}$

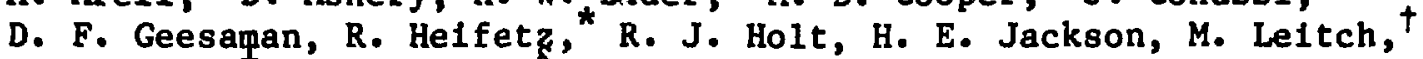

R. Redwine, ${ }^{\dagger} R$ E. Segel, J. R. Specht, K. E. Stephenson,

D. R. Tiegert, ||$^{\text {and }}$. Zupransk1

An experiment to study inclusive $\pi^{0}$ spectra from pion chargeexchange reactions with $160-\mathrm{MeV} \pi^{\ddagger}$ was performed using the pi-zero spectrometer at the LEP channel at LAMPF. This experiment is a continuation of our studies of inclusive pion charge exchange reactions which were previously carried out at lower energles. The $\pi^{\circ}$ spectra were measured at $0^{\circ}$, $40^{\circ}, 70^{\circ}, 110^{\circ}$, and $150^{\circ}$ on targets of ${ }^{12} \mathrm{C},{ }^{14} \mathrm{C},{ }^{58} \mathrm{NI},{ }^{120} \mathrm{Sn}$ and ${ }^{208} \mathrm{~Pb}$. Analysis of the measurements is under way. This experiment will establish the A dependence of the total charge-exchange cross sections. A comparison of the spectra of this experiment with those previously measured for inclusive pion Inelastic scattering should provide an estimate of the Importance of multistep processes. Furthermore, the quasifree mechanism should be strongly Inhibited at $0^{\circ}$ due to Pauli blocking.

\footnotetext{
*Tel Aviv University, Israel.

†Los Alamos National Laboratory, Los Alamos, New Mexico.

† Massachusetts Institute of Technology, Cambridge, Massachusetts.

$\$_{\text {Northwestern University, Evanston, Illinols. }}$

I Boston University, Boston, Massachusetts.
} 
f. Inclusive Reaction Cross Sections for $18,16^{O} O\left(\pi^{ \pm}, \pi^{\circ}\right)$

A. Are11, ${ }^{\star}$ D. Ashery, H. W. Bauer, ${ }^{\dagger}$ M. D. Cooper, ${ }^{\dagger}$ J. Conuzz1, ${ }^{\dagger}$

D. F. Geesaman, R. Helfetz, R. J. Holt, H. E. Jackson, M. Leitch, ${ }^{\dagger}$

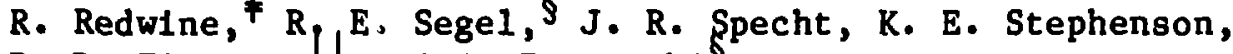

D. R. Tlegert, $\left.\right|_{\text {and P. Zupranski }}$

The inclusive differential cross sections for $\left(\pi^{ \pm}, \pi^{0}\right)$ reactions on ${ }^{16} \mathrm{O}$ and ${ }^{18} \mathrm{O}$ were measured at $160 \mathrm{MeV}$ with LAMPF $\pi^{\circ}$ spectrometer. A typical $\pi^{\circ}$ spectrum is shown in Fig. I-4. This experiment was designed to study the isospin dependence of the partial cross sectlons for inelastic scttering, charge exchange, and absorption. Previous measurements of the inelastic scattering and the sum of charge exchange + absorption show substantial differences in the distribution of reaction strength on these two targets. Data analysis is currently under way but a preliminary analysis suggests that the $\left(\pi^{+}, \pi^{\circ}\right)$ cross sections are larger on $18_{0}$ than $16_{0}$ and the $\left(\pi^{-}, \pi^{\circ}\right)$ cross sections are smaller on $18_{0}$ than $16_{0}$. This behavior is in qualitative agreement with predictions of the isobar-hole model. By combining these data with the ${ }^{12} \mathrm{C}$ and ${ }^{14} \mathrm{C}$ data described in the previous section, the dependence of these cross sections with the addition of p-shell nucleons will also be obtained.

\footnotetext{
* Tel Aviv University, Israel.

†Los Alamos National Laboratory, Los Alamos, New Mexico.

†assachusetts Institute of Technology, Cambridge, Massachusetts.

$\S_{\text {Northwestern University, Evanston, Illinois. }}$

II Boston University, Boston, Massachusetts.
} 


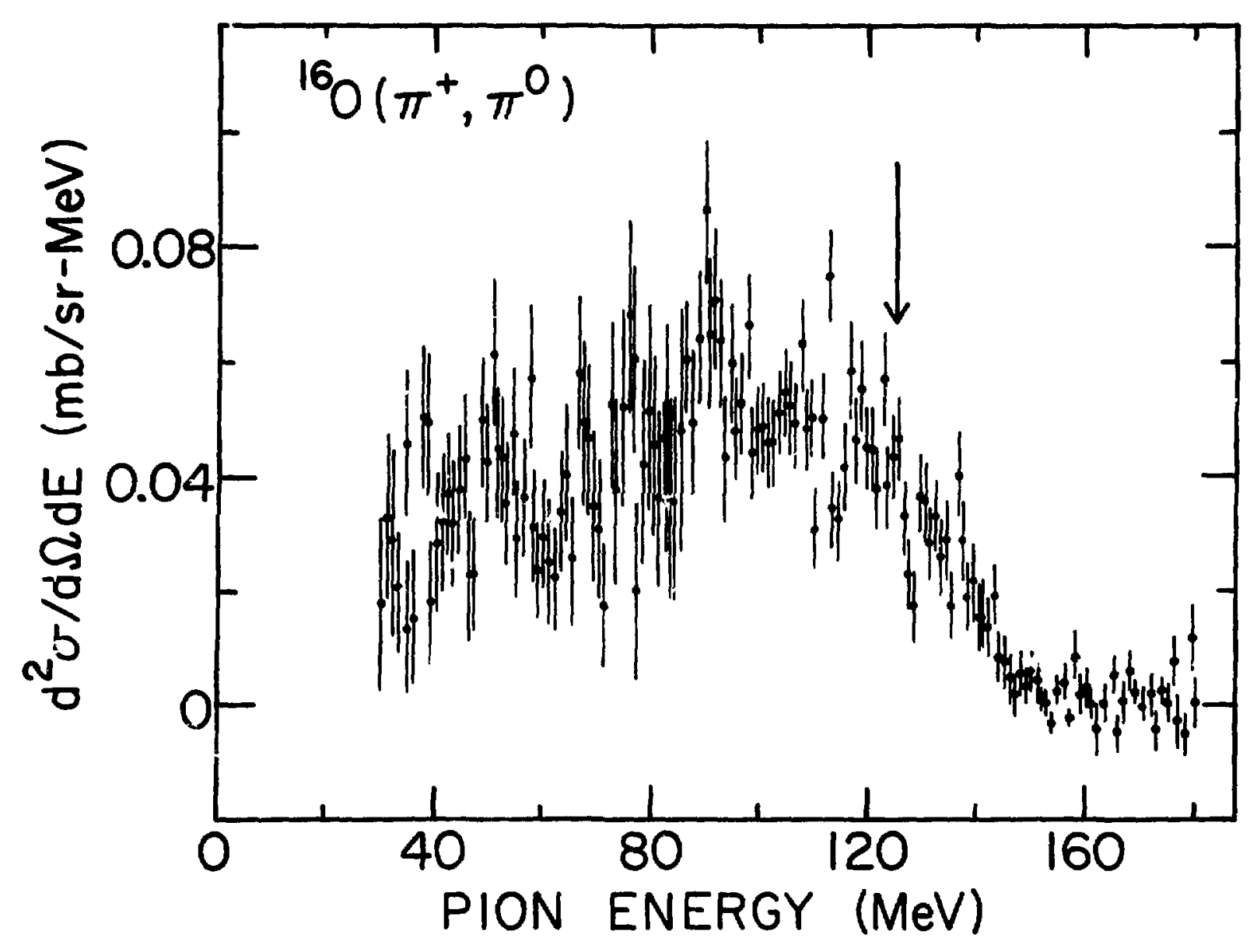

Fig. I-4. Spectrum for ${ }^{16} \mathrm{O}\left(\pi^{+}, \pi^{0}\right)$ at $70^{\circ}$ for $\mathrm{T}_{\pi}=160 \mathrm{MeV}$. The arrow indicates the approximate position for charge exchange on a free nucleon. 
g. Study of Plon Absorption in $3_{\text {He }}$ on and Above the $(3,3)$ Resonance

D. Ashery, D. F. Geesaman, R. J. Holt, H. E. Jackson, J. P. Schiffer,

J. R. Specht, K. E. Stephenson, B. Zeldman, R. Melnẹhart,

R. R. Whitney, ${ }^{*}$ G. Das, R. Madey, ${ }^{\dagger}$ B. D. Anderson, ${ }^{\dagger}$ and J. Watson ${ }^{\dagger}$

A proposal was submitted in which proton-proton angular correlation from the ${ }^{3} \mathrm{He}\left(\pi^{+}, 2 \mathrm{p}\right) \mathrm{p}$ reaction will be studied to determine the two-body ("quasi-deuteron") and three-body absorption cross sections. For each detection angle of one proton, the coincldent proton will be detected over a large solid angle; the measurement will be done for 4-5 detection angles. Proton-neutron angular correlation from the ${ }^{3} \mathrm{He}\left(\pi^{-}, \mathrm{pn}\right) \mathrm{n}$ reaction w1ll be atudied to determine the two-body absorption on the ${ }^{1} \mathrm{~S}_{0} \mathrm{~T}=1$ proton pair. Th1s measurement is complementary to the absorption studies on the $\left({ }^{3} \mathrm{~S}_{1} \mathrm{~T}=0\right)$ deuteron and bears upon the nucleon-nucleon interaction, the role of the $\Delta$ in the absorption process, and pion absorption in nucle1. The measurements wil? be performed in the $\mathrm{P}^{3}$ area ut1lizing the LAS spectrometer and four $40 \mathrm{in}$. $\times 10 \mathrm{fn} . \times 4 \mathrm{in}$. plastic scintillators. The measurements will be performed at 165, 24j, and $315 \mathrm{MeV}$ bombarding energles. Auxillary measurements of the ${ }^{3} \mathrm{He}\left(\pi^{+}, \pi^{+} \mathrm{p}\right)$ and ${ }^{3} \mathrm{He}\left(\pi^{-}, \pi^{-} n\right)$ will be done at one energy and one pion scattering angle in order to compare the relative momentum of a proton with respect to the $p-n$ pair and of a neutron with respect to the $p-p$ pair in $3_{\text {He. This is }}$ relevant for the absorption measurement where the momentum of the absorbing pair with respect to the third nucleon determines the width of the two nucleon angular correlation.

\footnotetext{
*Un1versity of Virginia, Charlottesville, Virginia.

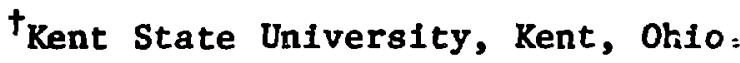




\section{h. Study of the $(\pi, \pi p)$ Reaction and Quasifree Scattering in ${ }^{4} \mathrm{He}$}

D. Ashery, D. F. Geesaman, R. J. Holt, H. E. Jackson, Jr.,

J. P. Schiffer, J. R. Specht, K. E. Stephenson, B. Zeldman,

R. E. Segel, ${ }^{\star}$ and $P$. Gram ${ }^{\dagger}$

Measurewent of Inelastic pion scattering observed in coincidence

with a recoll proton was proposed for a target of ${ }^{4} \mathrm{He}$. Angular distributions will be obtained for $\pi^{+}$at energies of 160,220 , and $350 \mathrm{MeV}$. Emergent pions wt11 be detected in the LAS spectrometer while proton detection will use an array of scintillation counters that span a large solid angle.

Data resulting from the coincident observation pions and recoll protons should provide a clear indication of the validity of the impulse approximation in describing the scattering and of the importance of contr butions from higher order processes.

\footnotetext{
*Northwestern University, Evanston, Illinois.

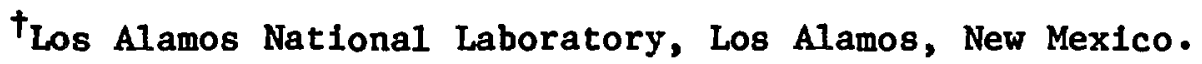

1. Study of Low-Energy Pion Absorption in ${ }^{3}$ He

A. Altman, ${ }^{*}$ J. Alster, ${ }^{*}$ K. Aniol, ${ }^{\dagger}$ D. Ashery, S. Mart $f n, *$

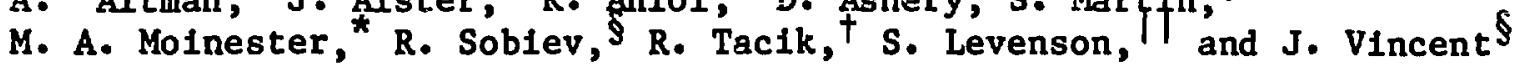

This experiment, planned for the spring of 1982 at TRIUMF, w111 measure the ${ }^{3} \mathrm{He}\left(\pi^{+}, 2 \mathrm{p}\right)$ and ${ }^{3} \mathrm{He}\left(\pi^{-}, \mathrm{pn}\right)$ reactions at $\mathrm{E}_{\pi} \leqslant 85 \mathrm{Mev}$. Absolute yields and angular distrthutions will be determined. The fraction of absorption that goes into three-body phase space ${ }^{3} \mathrm{He}\left(\pi^{+}, 3 p\right)$ will also be determined.

\footnotetext{
*Tel Aviv UnIver'sity, Israel.

tUniversity of British Columbia, Vancouver, B.C., Canada.

${ }_{\text {KFA, Julich, w. Germany. }}$

Suniversity of Toronto, Canada.

I Thesis student, University of Chicago, Chicago, Illinois.
} 


\section{j. Multiwire Proportional Chambers for LAS}

J. R. Specht, B. Zeidman, L. Atencio, ${ }^{*}$ and C. L. Mor:is ${ }^{*}$

A substantial improvement in the reliability of the LAS detector system was achleved by major modification and replacement of the multiwire proportional chambers. Modification in the construction of the first two chambers resulted in stable operation for both the chambers and the PCOS readout systen. The two rear chambers were replaced by multiwire proportional chambers that use delay-1ine readout. These new chambers, constructed with rigid metal frames, have active areas larger than 50 by $100 \mathrm{~cm}$.

\footnotetext{
${ }^{\star}$ Los Alamos National Laboratory, Los Alamos, New Mexico.
} 
B. NUCLEAR STRUCTURE STUDIES

The relationship between pion-nuclear interactions and nuclear structure is emphasized in these studies. While most of the program utilizes the EPICS system, certain problems are best studied with the LAS system. Occasionally, complementary data are obtained by scattering intermediate energy protons. Primary concerns are descriptions of inelastic scattering in terms of microscopic wave functions and the dynamics of pion-nucleon interactions. Differences between $\pi^{+}$and $\pi^{-}$scattering and analyses of the energy dependence of cross sections for inelastic pion scattering are related to particle-hole configurations and couplings between them. In other work, an upper limit for the cross section of a resonance in the four neutron system was investigated in a double-charge-exchange reaction on ${ }^{4} \mathrm{He}$.

a. Inelastic Pion Scattering from $10_{\mathrm{B}}$ and $11_{\mathrm{B}}$

B. Zeldman, D. F. Geesaman, C. Olnier, ${ }^{*}$ G. C. Morrison, ${ }^{\dagger}, G$. R. Burleson, ${ }^{\ddagger}$

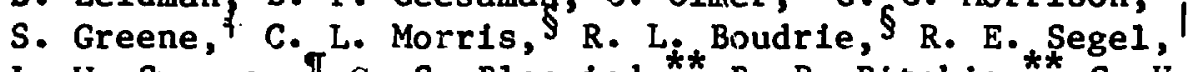

L. W. Swenson, $\pi^{\text {G. S. Blanpied, }{ }^{* *} \text { B. R. Ritchie, }{ }^{* *} \text { C. Harvey, }}{ }^{\dagger \dagger}$ and

P. Zupranski

Elastic and inelastic scattering of $\pi^{+}$and $\pi^{-}$by targets of $10_{B}$ and $11_{B}$ were investigated at $E_{\pi}=162 \mathrm{MeV}$. Angular distributions between $20^{\circ}$ and $90^{\circ}$ were measured in $5^{\circ}$ steps for $\pi^{+}$and $10^{\circ}$ steps for $\pi^{-}$. In addition, at $\mathbf{E}_{\pi}=130$ and $250 \mathrm{MeV}$, spectra were obtained at angles where the cross sections for angular momentum transfers, $\ell$, of $2,3,4$ are maximized. The energy dependence of the peak cross section (at fixed momentum transfer) is indicative of the spin dependence of the transitions, $1 . e .$, whether or not a

\footnotetext{
*Indiana Untversity, Bloomington, Indiana.

tUniversity of Birmingham, Birmingham, England

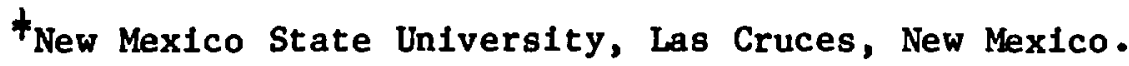

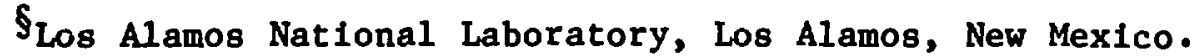

II Northwestern UnIversity, Evanston, Illinois.

"Oregon State UnIversity, Corvallis, Oregon.

** University of South Carolina, Columbia, South Carolina.

$t^{+}$University of Texas, Austin, Texas.
} 


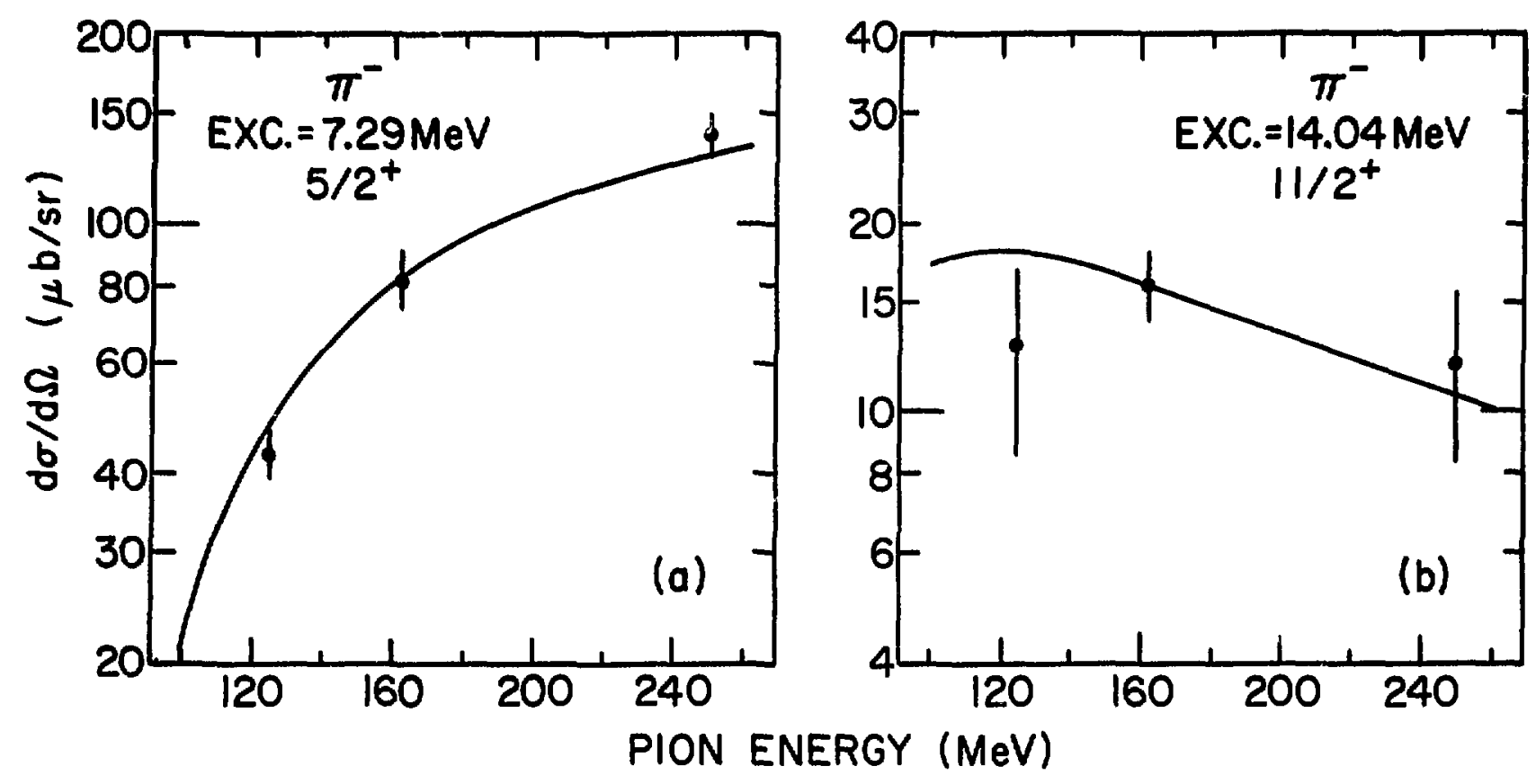

Fig. I-5. Energy dependence of peak cross sections for: (a) the $5 / 2^{+}$state at $E_{x}=7.29 \mathrm{MeV}$ in $11_{B}$, (b) the $11 / 2^{+}$state at $E_{X}=14.03 \mathrm{MeV}$ in $11_{B}$. The transition to the $5 / 2^{+}$state is primarily E3, while that to the $11 / 2^{+}$state is $\mathrm{M4}$. The curves are theoretical calculations.

spin-flip is involved. As the incident energy increases, the peak cross section should decrease for spin-flip transitions and increase for nonspinflip transitions. This effect is 1llustrated in Figure I-5. Together with the detailed angular distributions, the energy dependence of the cross sections should allow a decomposition of multipole contributions in excitations of states in $11_{B}$ which may proceed via mixed electric and magnetic multipoles. The results will be compared with theoretical shell-model predictions in a detalled test of both reaction models and nuclear structure. 
b. Inelastic Pton Scattering from ${ }^{14} \mathrm{~N}$

D. F. Geesaman, C. Olmer, ${ }^{*}$ B. Zeldman, G. C. Morrison, ${ }^{\dagger}$ G. Blanpled, ${ }^{\dagger}$

G. R. Burleson, R. L. Boudrle, R. E. Segel, $\prod_{\text {R. E. Anderson, }}$ and

L. W. Swenson"

Elastic and inelastic scattering of $163-\mathrm{MeV}$ pions from ${ }^{14} \mathrm{~N}$ was studled with the EPICS spectrometer at LAMPF. $\mathrm{CH}_{2} \mathrm{~N}_{2}$ and $\mathrm{CH}_{2}$ targets were employed at each angle, and the nitrogen spectra were constructed by subtraction. Many $14 \mathrm{~N}$ levels between 3 and $24 \mathrm{MeV}$ were observed, but only an upper limit could be set on the cross section for the $O^{+} \mathrm{T}=1$ state at 2.31-MeV excitation. Angular distributions were obtalned for 18 states. The majority of the states require $L=3$ transitions. Angular distributions for $5 \mathrm{~L}=3$ transitions are shown in Fig. I-6.

A state at $14.7 \mathrm{MeV}$ appears to be a $5^{-}$state which is predicted by shell-model calculations to be strongly excited in pion-inelastic scattering. Two additional states, at $16.4-$ and $17.5-\mathrm{MeV}$ excitation, may also be $5^{-}$states and show some evidence of isospin mixing. Microscopic DWIA calculations have some success in describing the states observed. The calculations typically underestimate the strength of the $\mathrm{L}=3$ transitions by a factor of 2, while the inelastic strength to the $14.7 \mathrm{MeV}$ state $1 \mathrm{~s}$ overestimated by about $30 \%$. The paper reporting the results of this work is in preparation.

\footnotetext{
*Indiana University, Bloomington, Indiana.

†niversity of Birmingham, Birmingham, England.

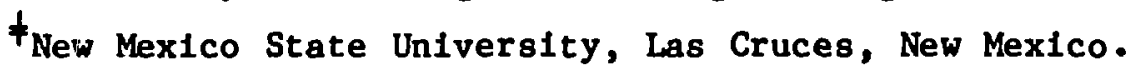

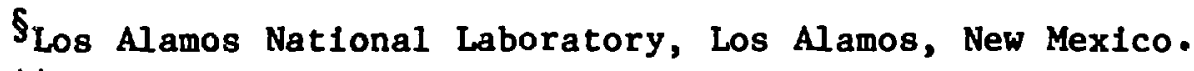

II Northwestern University, Evanston, Illinois.

TOregon State University, Corval11s, Oregon.
} 
Fig. I-6. Angular distributions for the $14 \mathrm{~N}\left(\pi, \pi^{\prime}\right)$ reaction to 5 negative parity states. The solid points denote the $\left(\pi^{+}, \pi^{+}\right)$cross sections, while the open points denote the $\left(\pi^{-}, \pi^{-\prime}\right)$ cross sections. The solid curves are the result of microscopic DWIA calculations, where the absolute normalization of the calculation has been adjusted to fit the data. The dashed curve is the result of a calculation in which $4 \%$ of the $4^{-}$amplitude to the 12.9-MeV state has been admixed into the $8.49-\mathrm{MeV}$ state.

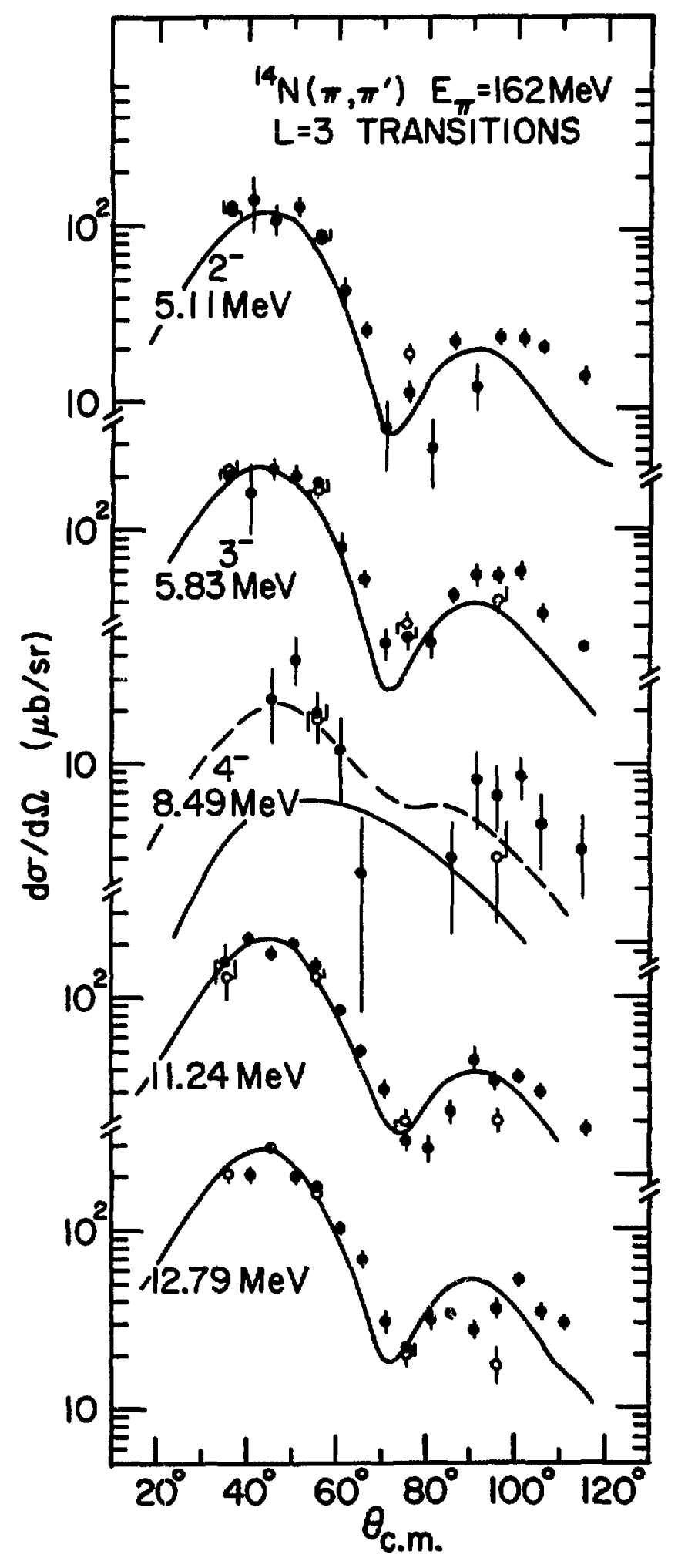




\section{c. Excitation of High-Spin Particle-Hole States in ${ }^{28} \mathrm{Si}$}

D. F. Geesaman, B. Zeldman, C. Ofmer, ${ }^{\star}$ s. Greene, ${ }^{\dagger}$ R. L. Boudrie, ${ }^{\ddagger}$

R. E. Segel, and L. W. Swenson

Inelastic pion scattering has proved to be an excellent tool for studying high-spin, particle-hole, spin-flip excitations in nuclel. In our earlier work at $162 \mathrm{MeV}$ on pion inelastic scattering to $\mathrm{T}=0$ and $\mathrm{T}=16^{-}$ states in ${ }^{28} \mathrm{SI}$ ( $11.58 \mathrm{MeV}$ and $14.34 \mathrm{MeV}$, respectively), we observed that the ratlo $R \equiv \sigma(T=0) / \sigma(T=1))$ was $\sim 1.5$ rather than the value of 4 expected from the pion-nucleon interaction. ${ }^{1}$ To test whether this discrepancy is due to the structure of the particular states, or a lack of understanding of the reaction mechanism, we repeated the experiment at $E_{\pi}=116 \mathrm{MeV}$. Spectra were accumulated at 65,80 , and $100^{\circ}$ with a $\pi^{+}$beam and $90^{\circ}$ with a $\pi^{-}$beam. At this energy the $6^{-}$states are much less prominent compared to other states in the spectra than at $162 \mathrm{MeV}$, and only an upper limit could be set on the yleld of the $6^{-} T=1$ state. The differences between the spectra at 116 and $162 \mathrm{MeV}$ can be understood in microscopic DWIA calculations as a result of the backward peaking of the angular distilbutions for natural parity states at pion energies around $100 \mathrm{MeV}$. The second maximum for $2^{+}$and $3^{-}$state angular distributions in ${ }^{28} \mathrm{~S} 1$ cocurs in the same angular region as the first maximum of the $6^{-}$ angular distribution. This explains why high-spin unnatural-parity states are most read1ly observed on-resonance $\left(E_{\pi}=162 \mathrm{MeV}\right)$ rather than below resonance as would be suggested from the energy dependences usually ascribed to spin-flip and nonspin-flip transitions.

\footnotetext{
*Indiana University, Bloomington, Indiana.

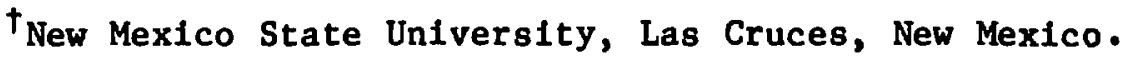

¥Los Alamos National Laboratory, Los Alamos, New Mexico.

$\S_{\text {Northwestern University, Evanston, Illinols. }}$

Iloregon State University, Corvallis, Oregon.
}

${ }^{1}$ C. Olmer, B. Zeidman, D. F. Geesaman, T.-S. H. Lee, R. E. Segel, L. W. Swenson, R. L. Boudrie, G. S. Blanpied, H. A. Thiessen, C. L. Morris,

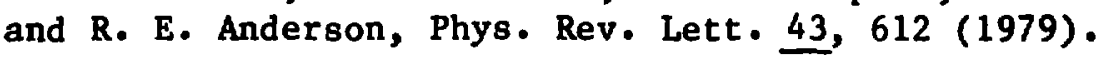


d. Determination of Neutron Rad11 from Plon Scattering

B. Zeldman and D. F. Geesaman

Despite obvious deficlencies in the theoretical analyses of pion scattering by complex nucle1, it was found that prescriptive modifications of the first order optical model for pion scattering on resonance yleld excellent agreement with high-quality scattering data. The strong isospin dependence on resonance implies a capability for differentlation of proton and neutron contributions to pion-nucleus scattering so that differences in rms radil can be deduced. The radius differences are in excellent agreement with the most recent values obtained from analyses of proton scattering. The results were presented in an invited paper and proceedings of a LAMPF User's meeting.

e. Excitation of High-Spin Particle-Hole States in ${ }^{54} \mathrm{Fe}$

B. Zeldman, D. F. Geesaman, R. D. Lawson, C. Olqer, ${ }^{*}$ R. L. Boudrfe, ${ }^{\dagger}$

C. L. Morfis, ${ }^{\dagger}$. A. Thlessen ${ }^{+}$R. A. Lindgren, G. R. Burleson ${ }^{\xi}$

S. Green, $\$$ W. B. Cottinghame, $\$$ R. E. Segel, $\{$ and L. W. Swenson

A high-resolution study of the inelastic scattering of $\pi^{+}$and $\pi^{-}$ by ${ }^{54} \mathrm{Fe}$ was proposed and approved. The principal objective is to locate and identify $8^{-}$states that arise from promoting a $1 f_{7 / 2}$ nucleon to the $1 g_{9 / 2}$ orbital. The EPICS system will be used in the initial measurements at $170 \mathrm{MeV}$. The experiment is scheduled for beam time in June 1982. Since high-spin states resulting from the excltation of stretched, particle-hole configurations are selectively populated at large momentum transfer, angular distributions consisting of relatively few, widely spaced data points of good statistical accuracy suffice for positive identification of the $8^{-}$states. Since ${ }^{54} \mathrm{Fe}$ has a neutron excess, both isoscalar and lsovector interactions contribute to excitation of $T=1$ states, but only

\footnotetext{
*Indiana University, Bloomington, Indiana.

${ }^{\dagger}$ Los Alamos National Laboratory, Los Alamos, New Mexico.

\#Un1versity of Massachusetts, Amherst, Massachusetts.

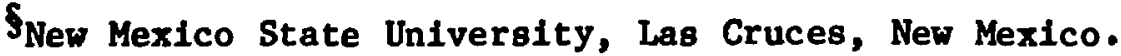

II Northwestern University, Evanston, Illinois.

Toregon State University, Corvallis, Oregon.
} 
the isovector interaction is involved in excitation of $T=2$ states. There should therefore be differences between $\pi^{+}$and $\pi^{-}$excitation of $T=1$ states, while the same cross sections are expected for $T=2$ states. She11model calculations suggest that pion scattering will populate states that are not observable in either proton or electron scattering.

f. Study of the ${ }^{4} \mathrm{He}\left(\pi^{-}, \pi^{+}\right)$Reaction at Small Angles

D. F. Geesaman, S. E. Green, * R. J. Holt, R. D. McKeown,

C. L. Morris, ${ }^{\prime}$ J. R. Specht, K. E. Stephenson, J. Ungar, ' and

B. Zeidman

A search for structure in the ${ }^{4} \mathrm{n}$ system via the ${ }^{4} \mathrm{He}\left(\pi^{-}, \pi^{+}\right)^{4} \mathrm{n}$ reaction in the energy range $130-200 \mathrm{MeV}$ was undertaken. The Argonne Large Acceptance Spectrometer (LAS) was used to detect $\pi^{+}$emerging from a liquid helium target located slightly upstream of the usual LAS target position. A 12 in. diameter circular dipole centered over the pivot deflected positive pions at $0^{\circ}$ toward LAS while deflecting the negative plons from the $\mathrm{P}^{3}$ beam away from the spectrometer. The efficiency and resolution of the system were determined by meacurements of ${ }^{12} \mathrm{C}\left(\pi^{ \pm}, \pi^{\mp}\right)$ double-charge exchange. Preliminary analysis of the data does not reveal any significant structure in the spectrum. Analysis is continuing in an effort to establish a rellable upper 11mit for the cross section for the formation of the tetra-neutron.

\footnotetext{
*New Mexico State University, Las Cruces, New Mexico.

†California Institute of Technology, Pasadena, California.

${ }^{\ddagger}$ Los Alamos National Laboratory, Los Alamos, New Mexico.
}

g. Discrete States from Plon Double-Charge Exchange on Heavy Nuclei

B. Zeldman, D. F. Geesaman, R. J. Holt, J. R. Specht, and K. E. Stephenson

The LAS spectrometer on the $\mathrm{P}^{3}$ channel will be used in a survey of pion double-charge exchange to discrete states on a wide variety of targets with $A>60$. In order to enhance the differential cross sections, the $\left(\pi^{+}, \pi^{-}\right)$reactions w111 be studied near $0^{\circ}$ with $\sim 150-$ MeV pions. The 
feasibility of the technique, which uses a small dipole to deflect the primary beam away from the spectrometer, was demonstrated during the setup for the ${ }^{4} \mathrm{He}\left(\pi^{-}, \pi^{+}\right)$experiment. Unfortunately, an equipment fallure in the $\mathrm{P}^{3}$ channel curtailed the run so that this experiment will be rescheduled in 1983. It is antictpated that a tune providing a dispersed beam at the target will result in improved resolution for this experiment.

h. Dispersed Beam for LAS

B. Zeidman

With the present tune of the $\mathrm{P}^{3}$ channel at LAMPF, experiments utilizing the Argonne Large Acceptance Spectrometer (LAS) have energy resolutions which are limited by the total momentum bite accepted for transmission. The resolving power of LAS is substantially better than can be achieved with the achromatic tune of the $\mathrm{P}^{3}$ channel. Calculations show that a dispersed beam tune can provide a resolution of $<0.5 \%$ on target with a total channel acceptance of $2 \%$, thereby maintaining beam intensity. Implementation of this tune will allow significantly improved resolution in double-charge exchange experiments already approved for LAS and also permit moderate resolution studies of pion scattering experiments at $E_{\pi} \approx 550 \mathrm{MeV}$. This information has been transmitted to interested users.

\section{Polarized Proton Scattering from ${ }^{26}{ }_{\mathrm{Mg}}$}

D. F. Geesaman, B. Zeldman, C. Olmer, * A. D. Bacher, ${ }_{*}^{*}$ G. T T Emery, ${ }_{t}$

C. W. Glover, ${ }^{*}$ H. Nann, ${ }^{*}$ W. J. Jgnes, ${ }^{*}$ S. Y. van der Werf,,

R. E. Segel, and R. A. Lindgren ${ }^{\S}$

We have measured the angular distributions and analyzing powers for polarized proton scattering from ${ }^{26} 6_{\mathrm{Mg}}$ at $135-\mathrm{MeV}$ incident energy at the IUCF (Indiana University Cyclotron Facility). Angular distributions were measured in $5^{\circ}$ steps from a laboratory angle of $10^{\circ}$ to $60^{\circ}$ for states in

\footnotetext{
*Indiana University, Bloomington, Indiana.

$t_{K V I}$, University of Groningen, The Netherlands.

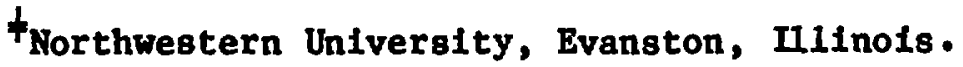

Suntversity of Massachusetts, Amherst, Massachusetts.
} 
the excitation energy range from 0 to $20 \mathrm{MeV}$. Data analysis is currently under way.

This experiment is a continuation of our efforts to understand the quenching of spin-filp strength in high-spin particle-hole states. The phenomenón is now well established throughout the periodic table for $\Delta T=1$ transitions, and is also observed in $\Delta T=0$ transitions in pion and proton scattering. By studying the inelastic strength as a function of deformation in the $s-d$ shell, and as a function of angular momentum transfer, we hope to distinguish between several proposed explanations for the quenching mechanism, Including the possibility of isobar-hole admixtures in the nuclear wave functions.

\section{j. Search for Mesonic Effects on Inelastlc Proton Scattering to the} $10.24-\mathrm{MeV} 1^{+}$state $\ln { }^{40} \mathrm{Ca}$

K. E. Rehm, P. Kienle, ${ }^{*}$ D. W. Miller, ${ }^{\dagger}$ R. E. Segel,,$^{\ddagger}$ and J. R. Comfort

With special emphasis on exciting unnatural parity states, differentital cross sections and analyzing powers were measured for inelastic scattering of $160-\mathrm{MeV}$ polarized protons on ${ }^{48} \mathrm{Ca}$. A strong excitation of the $10.23-\mathrm{MeV} 1^{+}$state is observed at small scattering angles. DWIA calculations using shell-model wave functions which fit Inelastic electron scattering form factors over-predict the cross section of small $q$ by about a factor of 2 . The results are consistent with a $q-$ dependent modification of magnetic transitions as predicted from virtual $\Delta$-hole excitations.

\footnotetext{
*T.U. München, West Germany.

tIndiana University Cyclotron Fac1l1ty, Bloomington, Indiana.

†orthwestern University, Evanston, Illinois.

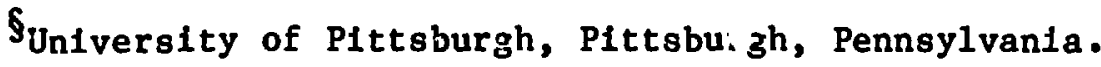




\section{TWO-NUCLEON PHYS ICS WITH PIONS AND ELECTRONS}

Studies of the deuteron in the medium-energy range are expected to place significant constraints on our knowledge of the reaction mechanisms as well as the basic nucleon-nucleon interaction. Two of the major 1ssues which are closely related to $\pi-d$ scattering are the effects of pion absorption and dibaryon resonances on the elastic channel. The signatures for these effects show up most clearly in the polarization observables in $\pi-d$ scattering. Elastic electron scattering from the deuteron is expected to be sensitive to the nucleon-nuclecn interaction at short range and the meson-exchange current. Again, these effects are expected to be most clear in the tensor polarization. Experiments are being designed which will provide measurements of the tensor polarization For broad ranges of momentum transfer in both $\pi-d$ and $e-d$ elastic scattering.

a. Measurement of the Angular Distribution of Tensor Polarization in $\pi-d$ Elastic Scattering

D. F. Geesaman, R. J. Holt, J. R. Specht, K. Stephenson, B. Zeidman, J. S. Frank, ${ }^{*}$ M, J. Leitch, , J. D. Moses, * E. J. Stephenson, ${ }^{\dagger}$ and R. M. Laszewski ${ }^{*}$

During the past four years a number of theoretical calculations of polarization in $\pi-d$ elastic scattering have appeared. These calculations have indicated that the polarization observables, especially the tensor polarization $t_{20}$, are particularly sensitive to two effects, pion absorption and possible dibaryon resonances. ${ }^{2}$ At SIN, oscillations in the angular dependence ${ }^{3}$ of the vector polarization have been observed for $\mathrm{T}_{\pi}=256 \mathrm{MeV}$. These oscillations suggest the presence of dibaryon resonances. However, an absorption effect cannot be eliminated on the basis of those data alone. Thus, we have proposed to measure the angular dependence of $t_{20}$ at $T_{\pi}=180$ and $256 \mathrm{MeV}$.

\footnotetext{
*Los Alamos National Laboratory, Los Alamos, New Mexico.

†Indiana University Cyclotron Facility, Bloomington, Indiana.

\#University of Illinois, Urbana, Illinois.

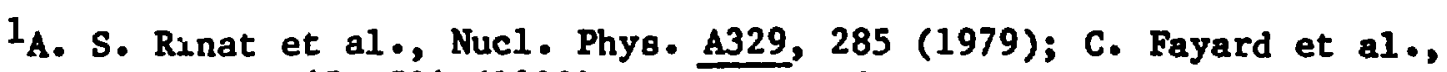
Phys. Rev. Lett. 45, 524 (1982); M. Betz and T.-S. H. Lee, Phys. Rev. C 23, 375 (1981); B. Blankleider and I. R. Afnan, Phys. Rev. C 24, 1572 (1981).

${ }^{2}$. Kubodera et al , J. Phys. G 6, 171 (1980).

3. Bolger et al., Phys. Rev. Lett. 46, 167 (1981).
} 
We have measured the angular dependence ${ }^{4}$ of $t_{20}$ in $\pi-d$ Bcattering at $T_{\pi}=142 \mathrm{MeV}$. The results were found to be in disagreement with all present theoretical predictions. The disagreement near $\theta_{\pi}=98^{\circ} 1 \mathrm{~s}$ particularly interesting since $t_{20}$ has little sensitivity to the deuteron wave function or the absorption channel at that angle. The inclusion of dibaryon resonances in the calculation results in better agreement with the datum at $98^{\circ}$.

${ }^{4}$ R. J. Holt et al., Phys. Rev. Lett. 47,472 (1981).

b. Tensor Polarization in Electron-Deuteron Elastlc Scattering

R. J. Holt, J. R. Specht, K. E. Stephenson, B. Zeldman, $w_{i}$ Haeberl1, *

W. Bertozzi, ${ }^{+}$R. P. Redwine ${ }^{+} M$. Schulze, ${ }^{+}$W. Turchinetz, ${ }^{\dagger}$

R. Galoskle, \$ D. W. Saylor, E. J. Stephenson, \$ R L. Burman, $\neq$

J. S. Frank, ${ }^{\neq}$. J. Leitch, ${ }^{\ddagger}$ and R. M. LaszewsleI

It 1 sell known that only three form factors (charge, quadrupole, and magnetic) are necessary to describe e-d elastic scattering. Unfortunately, cross-section measurements alone do not allow one to unravel these three form factors. Thus far, only the magnetic form factor has been isolated. However, the charge anc quadrupole form factors are sensitive to the short-range and tensor part, respectively, of the nucleon-nucleon interaction. The tensor polarization $t_{20}$ is dominated by the interference term between the charge and quadrupole form factors.

We have proposed to measure $t_{20}$ in e-d scattering at the MIT/Bates accelerator. The first phase of this measurement spans the momentum transfer range $q^{2}<9 \mathrm{fm}^{-2}$. The second phase depends on the avallability of the electron beam recirculator at Bates and will cover the momentum transfer range: $9<\mathrm{q}^{2}<14 \mathrm{fm}^{-2}$.

\footnotetext{
*University of Wisconsin, Madison, Wisconsin.

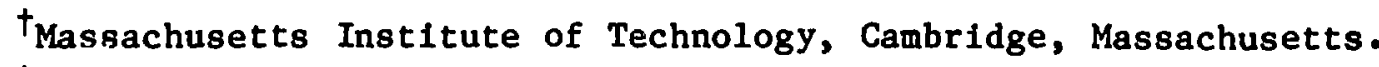

${ }^{\ddagger}$ Los Alamos National Laboratory, Los Alamos, New Mexico.

Indiana University, Bloomington, Indiana.

I University of Illinois, Urbana, Illinois.
} 
A feasibility test was performed this year. It was learned that the rate of photo-produced deuterons from ${ }^{16} \mathrm{O}$ in the $\mathrm{D}_{2} \mathrm{O}$ target was too high for the polarimeter. Th1s means that efther a pure liquid deuterlum target or an electron tagging spectrometer are necessary for the experfment. Since a large acceptance spectrometer (OHIPS) is under construction already at MIT, it was decided to perform the experiment after the completion of the spectrometer.

c. Feasibility Study of Electron Scattering Experiments with a Polarized Target in an Electron Storage Ring

R. J. Holt, C. Gagliard1, L; S. Goodman, J. R. Specht, K. Stephenson, B. Zeidman, and J. D. Moses

A study is belng carrled out to determine the feastbility of measuring tensor polarization in e-d scattering with the use of the high circulating electron currents in an electron ring and a polarized atomic beam source. The expected advantages of performing this experiment with a storage ring are (1) high circulating current, which implies that thin targets such as polarized atomic gas targets can be employed, (11) an essentially $100 \%$ duty factor electron beam with a substantially higher energy than is avallable at the medium-energy electron facilities, (iii) low background rate, which implies that the deuteron or electron detectors and shlelding can be greatly simplified, and (Iv) the use of a polarized atomic target, which unlike a polarimeter experiment, allows the spin state to be readily varied so that the measurements should have greater accuracy. The study is being performed in two stages. First, it must be determined whether or not it is practical. to scatter electrons from a fixed gas jet target in a storage ring. Then, secondly, it must be demonstrated that tensor polarized targets of sufficient thicknesses can be attained.

This study is centered around the Aladdin storage ring at Stoughton, W1sconsin. The expected characteristics of this ring are a c1rculating current of $\geqslant 100 \mathrm{~mA}$ and an electron energy of $1.0 \mathrm{GeV}$. The

\footnotetext{
*Los Alamos National Laboratory, Los Alamos, New Mexico.
} 
study 1nvolves developing a polarized target of areal density of $10^{12}$ to $10^{13}$ atoms $/ \mathrm{cm}^{2}$. This appears to be feasible with existing technology.

\section{d. Deuteron Tensor Polarimeter Development}

R. J. Holt, J. R. Specht, E. J. Stephenson, * K. E. Stephenson, J. D. Moses, ${ }^{\dagger}$ R. P. Redwine, ${ }^{+}$M. Schulze, ${ }^{+}$and w. Turchinetz ${ }^{*}$

A high efficlency deuteron tensor polarlmeter was used to measure the angular distribution of $t_{20}$ in $\pi-d$ scattering at $T_{\pi}=142 \mathrm{MeV}$. This polarimeter will be used to measure the tensor polarization in $\pi-d$ scattering at $T_{\pi}=180$ and $256 \mathrm{MeV}$. In addition, 1 t w11l be used to measure the tensor polirization in e-d scattering at low momentum transfer. For this purpose the polarimeter must undergo some minor modifications and be recalibrated. The efficiency of the polarimeter will be measured with the three-stage tandem Van de Graaff at the Los Alamos National Laboratory.

A proposal was made to the Indiana University Cyclotron Laboratory to survey tensor analyzing powers in high-energy deuteron reactions. The purpose of this search is to find a reaction sultable for a high-energy deuteron tensor polarimeter. If a high energy deuteron tensor polarimeter is feasible, it would greatly simplify the measurements of tensor polarization in e-d scattering at high momentum transfer.

\footnotetext{
*Indiana University, Blooming,ton, Indlana.

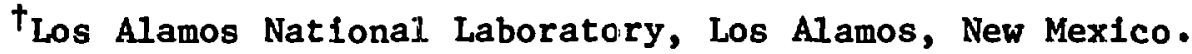

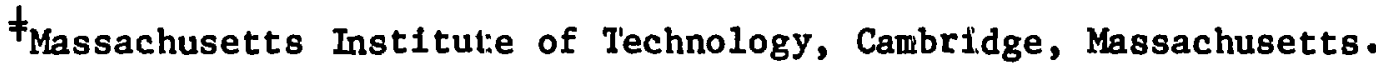




\section{NEUTRINO OSC ILLAT IONS}

\section{Neutrino 0sciliations at LAMPF}

G. T. Garvẹy, C. Gagliard1, $s_{f}$ Freedman, ${ }^{*}$ c. L. Hyman, ${ }^{\dagger}$ B. Musgrave, ${ }^{\dagger}$

T. Y. Ling, ${ }^{\ddagger}$ F, A. Romanowsk1, ${ }^{*}$ R. Imlay, W. J. Metcalf, $\$$ and

R. D. McKeown

The possibility and consequences of $v$ osctllations has recently recelved much attention ${ }^{1}$ because of developments in the extension of gauge theorles to include strong and electroweak interactions. These new Grand Unifled Theorles (GUTS) rather naturally produce massive $v$ 's and flavor mixing in the lepton sector among other striking predictions. We are part of a collaboration with an approved experiment (E645) at LAMPF to search for $v$ oscillations. The neutrino source is to be the LAMPF beam dump which yields $\nu_{e}, \nu_{\mu}$ and $\tilde{\nu}_{\mu}$ neutrinos from stopped decays.

The energies of the $\nu$ 's produced are sufficlently low that only $v_{e}$ or $\tilde{\nu}_{e}$ can participate in charge changing interactions. Two kinds of oscillation experiments are to be performed. In one the disappearance of electron neutrinos will be measured by observing the spatial dependence of the neutrino flux with the reaction $\left(v_{e}+d\right) \rightarrow\left(e^{-}+2 p\right)$. In the absence of $v_{e}$ oscillations, the number of $v$ measured will be inversely proportional to the square of the source-to-detector distance. Neutrino mixing will produce oscillations in the variation of flux with distance, the period of this oscillation being determined by the mixing angle between neutrino types and the mass difference. The low energy of the LAMPF $\nu_{e}$ 's makes it attractive to search for oscillations over practical distances. The other

*Stanford University, Stanford, California.

${ }^{+}$High Energy Physics Division, ANL.

¥Ohio State University, Columbus, Ohio.

SLulsiana State University, Baton Rouge, Louisiana.

IICalifornia Institute of Technology, Pasadena, California.

$1_{M}$. Magg and Ch. Wetterich, Phys. Lett. 94B, 61 (1980); A. Zee, Phys. Lett. 93B, 389 (1980); R. N. Mohapatra and G. Sen janovič, Phys. Rev. Lett. $44,91 \overline{2}(1980) ;$ and R. N. Mohapatra and R. E. Marshak, Phys. Rev. Lett. 44 , 1316 (1980). 
phase of this experiment involves the appearance of $\tilde{v}_{e}$ electrons $v i a$ $\tilde{v}_{\mu}+\tilde{v}_{e}$; the $\tilde{v}_{e}$ being detected via $\left(\tilde{v}_{e}+p\right)+\left(e^{+}+n\right)$. During the past year efforts of the ANL participants have been devoted to critically examining the physics and sechnical issues in this experiment. A 5 ton and a 15 ton detector to perform these experiments have been designed. The detector is a fine grained $\left(\sim 2.5 \mathrm{gm} / \mathrm{cm}^{2}\right)$ matrix of Cherenkov or scintillator detectors and drift chambers and is shown in Fig. I-7. Some testing of components for the main detector will go on in this fiscal year but the principal task is starting construction of the entire detector system in a timely fashion.

ANL has assumed responsibility for the cosmic-ray anticoincidence shield and overall engineering for the experiment. The shield is crucial to the success of the experiment as there are some $10^{5}$ cosmicray mesons stopped in the detector per LAMPF day. This number must be reduced by a factor of $10^{5}-10^{6}$ to achleve the low background required to push the oscillation search to its limits. 
I5 TON $\nu$ DETECTOR MODULE

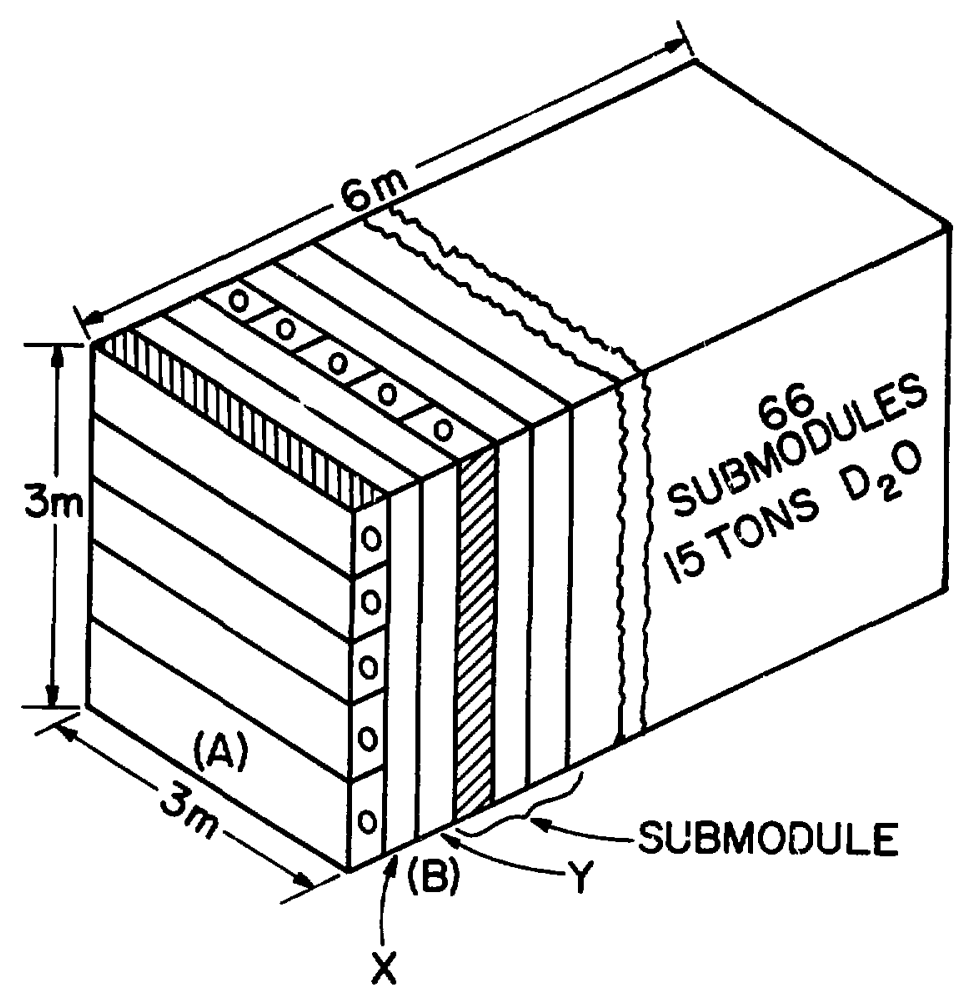

(A) $\mathrm{D}_{2} \mathrm{O}$ RADIATOR

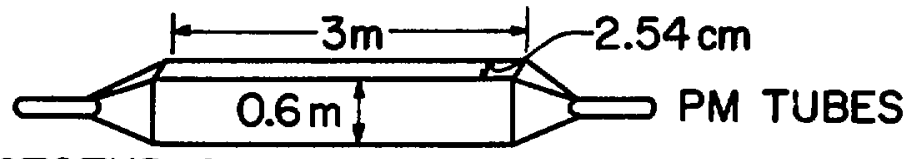

PHOTOTUBES

AMPEREX 2212/B

(12 STAGE) 2/SLAB

(B) PROPORTIONAL DRIFT CHAMBERS

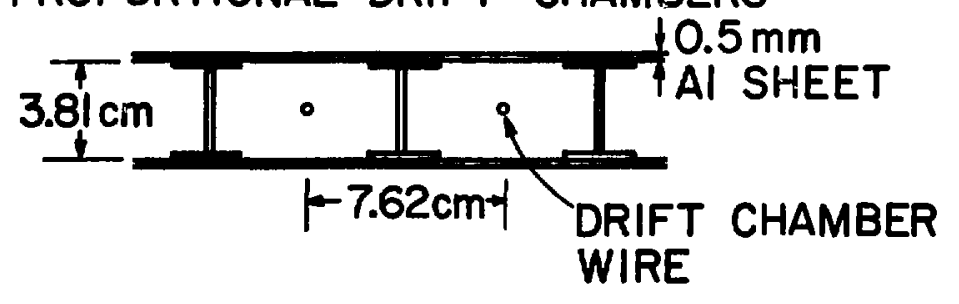

F1g. I-7. Schematic drawing of the 15 ton $v$ detector for LAMPF-E645. 
1 


\section{HEAVY-ION RESEARCH AT THE TANDEM AND \\ SUPERCONDUCTING LINAC ACCELERATOR}

INTRODUCTION

The superconducting booster, injected by the FN tandem, is now completed and provides heavy-ion beams up to mass $A \approx 80$ with energies sufficient to surmount the Coulomb barrier of even the heaviest target nucleus. This allows us to extend the heavy-ion research program in the Argonne Physics Division which is principally concerned with the many facets of the relationships between heavy-ion induced reactions and nuclear structure. Major interests are the structure of nuclei at high excitation, far from stability or in states of high spin near and above the yrast line.

Considerable new structure information on high angular momentum states has been obtained in discrete and continuum $\gamma$-ray studies near and above the yrast line. One aim of this program is to elucidate the structure of nuclei as a function of spin, neutron number and excitation energy above the yrast line, all within one common framework. The relevant experimental work bears on identifying very high spin yrast states and on extracting their properties, i.e., collective or single-particle nature, lifetimes, etc. Systematic discrete $\gamma$-ray studies on yrast states of predominantly aligned particle configurations, of transitional nuclei with interplay between aligned-particle structure and collective rotation, and on nearclosed shell nuclei have been performed. Continuum $\gamma$-ray cascades provide information on excited states above the cold yrast lines. Both discrete and continuum spectroscopy studies point towards shape changes as a function of spin, neutron number, and excitation energy.

For light and medium weight systems and at bombarding energies not too far above the Coulomb barrier, fusion of heavy ions to form a compound system accounts for a major fraction of the total reaction cross section. With increasing projectile and compound nucleus mass the fusion cross section decreases rapidly and seems to disappear completely for the heaviest systems. Previous detailed studies at Argonne on fusion in light systems has revealed a dependence of the maximum cross section for fusion upon the nuclear structure of the colliding nuclei. The current fusion program is aimed at studying whether a similar behavior exists for the much heavier system, thus possibly pointing the way for the production of nuclei far off stability by a suitable choice of target and projectile nuclei. For a comparison of fusion behavior with predictions of statistical models, the evaporation channels have been studied in measurements of neutron and characteristic $x$-ray spectra. Singles and multiplicity measurements of the latter provide an unambiguous identification of the charge distribution of the evaporation residues.

The distribution of the reaction strength into the various reaction channels bears on the mechanisms governing the interaction between two complex nuclei. In addition to complete fusion, the incomplete fuston processes, fusion-fission, deep-inelastic and quasielastic scattering processes 
are of considerable interest. Measurement of momentum spectra of the reaction products can help to distinguish between different reaction mechanisms which are intertwined with nuclear structure effects.

The observation of resonance-like structure in quasielastic reactions on $s-d$ shell nuclei has generated considerable interest in the underlying nature of such behavior. Searches have been performed for resonant structures in heavier systems, for signatures of resonant exchange between nearly degenerate channels, and for signs of massive transfer to quasimolecular configurations. The observation of resonances in only selected systems points to specific nuclear structure aspects underlying their occurrence.

The excellent accelerator properties are exhibited in high-sensitivity mass spectrometry measurements that allow detection of minute quantities of radioisotopes in macroscopic samples. Measurements of otherwise difficult to obtain nuclear cross sections of specific interest, nuclear lifetimes, cross sections related to the solar neutrino problem, exotic particles, etc. can be performed with this technique which is possibly the only efficient method.

Major steps have been taken towards the completion of the new target area for the superconducting linac booster. Installation of the split-pole spectrograph has been completed and the instrument has been used in first measurements. A thin-walled neutron time-of-flight chamber has been installed in a new beam line and undergone first tests. A laser system for optical hyperfine-structure measurements, with a cryogenic helium jet for fast transport of nuclear reaction products into the laser interaction region has been set up and first tests performed. Several gas counters have been developed and used in charged-particle measurements. An electrostatic deflector has been built and used in complete fusion measurements in heavy systems. Design for an additional small beam line to house an electron spectrometer is under way. A plunger for recoil-distance lifetime measurements has been constructed and used in initial measurements. 


\section{HIGH ANGULAR MOMENTUM STATES IN NUCLEI}

One aim of this program is to elucidate the structure of nuclei as a function of spin, neutron number and excitation energy above the yrast 1ine. We have systematically studied the Dy isotopes and have established that the yrast lines of the isotopes with $\mathrm{N}=82-86$ are dominated by aligned-particle configurations and are oblate. We are now concentrating on the transitional isotopes with $N=87$ and 88 in order to study the transition between the aligned-particle structures and the prolate collective rotational structures. Another aspect of interest concerns the excited states above the "cold" yrast line. Here experiments focus on the properties of continuum $\gamma$-ray cascades which feed the yrast states. Both our discrete and continuum spectroscopy studies point towards shape changes as a function of spin, neutron number, and excitation energy. A different aspect of our work concerns the spectroscopic investigations of $\mathrm{N}=82$ nuclei with a few $(2-6)$ protons in excess of $Z=64$. The goal here is to compare the $\left(h_{11 / 2}\right)^{n}$ structures to shell-model calculations which assume a $Z=64$ core. The relevant experimental work involves spectroscopy of nuclei near the proton drip line.

The $\gamma$-ray facility is being steadily expanded. A plunger for lifetime measurements has been constructed and is now ready for measurements. A superconducting solenoid lens electron spectrometer will be constructed by the Purdue group.

\section{a. Transition from Collective to Aligned-Particle Configuration at High Spin in $154 \mathrm{Dy}$}

A. Pakkanen, ${ }^{\star}$ I. Ahmad, ${ }^{\dagger}$ P. Chowdhury, Y. H. Chung, ${ }^{*}$ P. J. Daly, * S. R. Faber, * Z. Grabowski, H. Helppi, * T. L. Khoo, and J. Wilson*

The yrast configurations of nuclel with $N \geqslant 90$ are known to be generated from the collective rotation of a prolate shape. On the other hand, those for nuclei with $N \leqslant 86$ are formed from the alignment of high-j particles, which leads to an oblate mass distribution at high spins. Both prolate and oblate shapes arise from shell-structure effects. In the transitional nuclei with $\mathrm{N}=87$ and 88 both types of shell effects should compete, providing an important testing ground for theory.

We have completed extensive in-beam $\gamma$-ray spectroscopic studies of ${ }^{154}$ Dy $(N=88)$ using the $\left({ }^{34} \mathrm{~S}, 4 \pi\right)$ reaction. The high spin yrast levels have been established up to spin 34 or 35 (see Fig. II-1). The lifetimes of yrast states have also been measured using the recoil distance method. The

${ }^{\star}$ Purdue University, West Lafayette, Indiana.

${ }^{+}$Chemistry Division, ANL. 


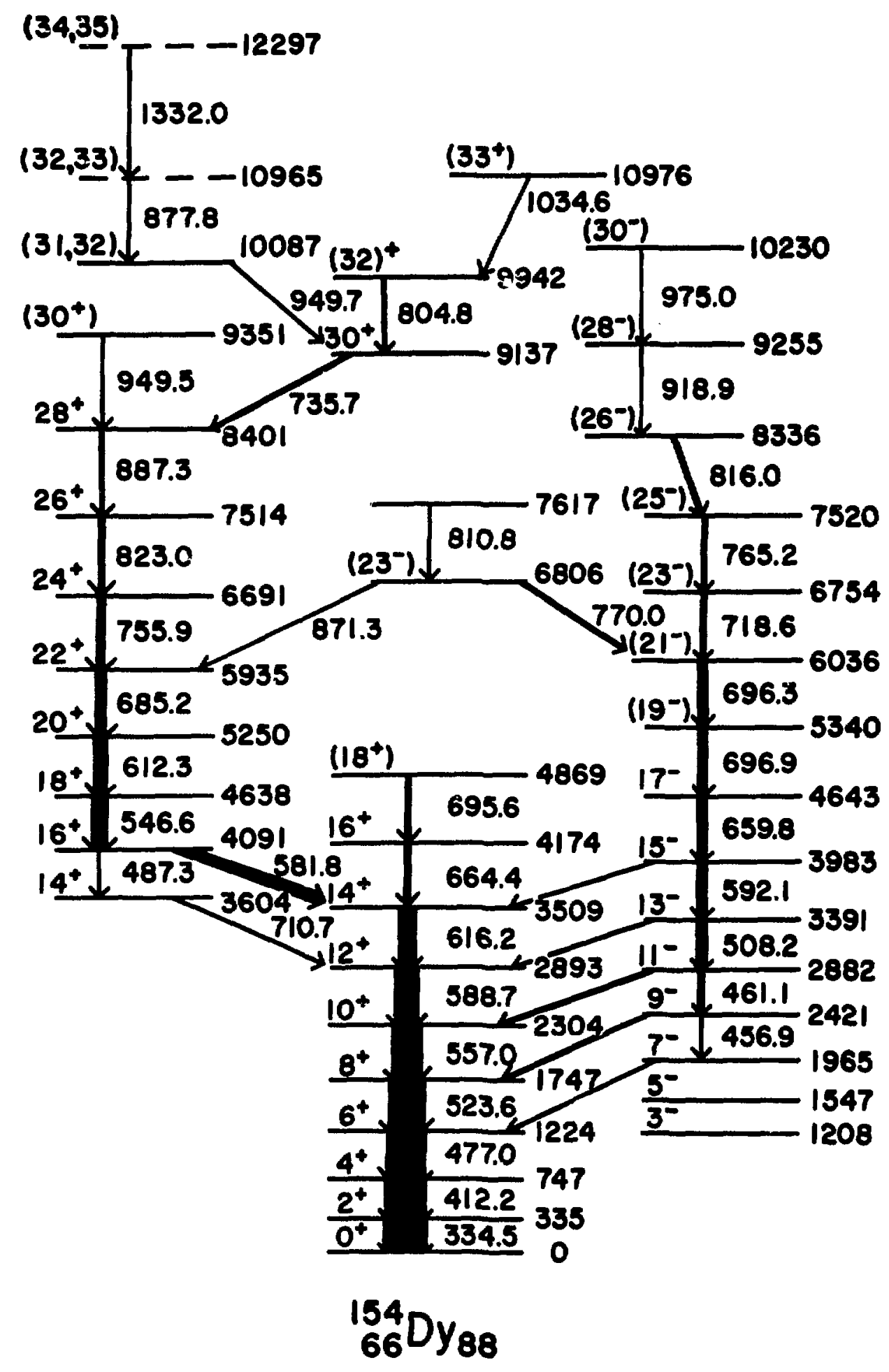

Fig. II-1. Level scheme of ${ }^{154}$ Dy. 
level structure, $\gamma$-ray deexcitation modes and lifetimes indicate the following. Up to spin 32, the yrast levels arise from collective rotation; there are two backbends at $I=16$ and 30 , due to the rotation-alignment of high-j particles. Above this spin a transition to aligned-particle structure occurs, probably giving rise to oblate shapes. Hence, for the first time, 1 a prolate-to-oblate transition has been observed in a heavy nucleus. The lifetimes suggest that this transition occurs through a series of triaxial shapes.

It has also been possible to extract the amount of spin alignment of the $i_{13 / 2}$ particle along the rotation vector as a function of rotational frequency. We are currently investigating the role of this alignment in the prolate-to-oblate shape change and also in the onset of prolate deformation with increasing neutron number. The goal is towards an understanding of the spin- and neutron-number dependence of nuclear structure.

${ }^{1}$ A. Pakkanen et al., Phys. Rev. Lett. $\underline{48}, 1530$ (1982).

b. High Spin Structure of ${ }^{153}$ Dy

M. Kortelahti, " P. Chowdhury, Y. H. Chung, , P. J. Daly, , Z. Grabowski,

R. V. F. Janssens, T. L. Khoo, J. McNeill, * and A. Pakkanen*

Our systematic studies of Dy isotopes have shown that isotopes with $\mathrm{N}<86$ have yrast configurations formed from aligned particle configurations. On the other hand, the isotopes with $N \geqslant 90$ are known to be prolate rotors. Our recent investigations of $\mathrm{N}=88^{154}$ Dy show a transition from collective to aligned-particle character at high spin $(I>32) .^{1}$ We also expect the interplay of collective and aligned-particle structures in the $N=87$ isotope.

To verify this we have begun investigating the high-spin structure in ${ }^{153}$ Dy using the $\left({ }^{34} \mathrm{~s}, 4 \mathrm{n}\right)$ reaction. Data analysis is still proceeding. Preliminary indications are that a change occurs from a smooth yrast line to an irragular one around a spin of $\geq 41 / 2$. In addition, we have identified isomers with half-lives of 21.5 ns decaying into an $1_{13 / 2}$ band, which appears rotation-aligned. When this study is complete, we shall have valuable data for examining, in a unified framework, the role of spin and neutron

* Purdue University, West Lafayette, Indiana.

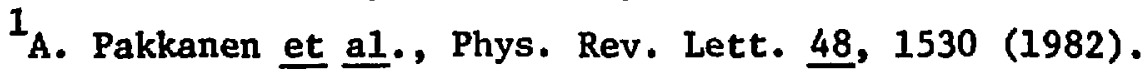


number in the nuclear structure of transitional nuclei. Only angular distribution and recoil distance measurements remain to be performed.

c. Feeding of High-Spin Particle Yrast States in 151,152 Dy by Collective Cascades

P. Chowdhury, S. R. Faber, ${ }^{*}$ I. Ahmad, ${ }^{\dagger}$ J. Borggreen, Y. H. Chung, ${ }^{*}$ P. J. Daly, * R. K. Smither, and J. Wilson*

The yrast states of ${ }^{152} \mathrm{Dy}$, which we have identified up to spin 40 , are dominated by aligned-particle configurations, However, the nature of the states above the yrast line and at higher spin remains to be ascertained. To this end we have observed the population-pattern of the yrast states and the continuum spectra from the $\gamma$ cascade feeding these states. The $\gamma$ cascade has a large E2 component around $1.3 \mathrm{MeV}$, and we have mapped the average energy of this component as a function of the input angular momentum by means of a sum spectrometer. It was then possible to infer the average $\gamma$-deexcitation trajectories, which might be associated with collective structures built on the aligned-particle states. These collective structures may be associated with triaxial and, at the highest spin, prolate shapes.

The first phase of these studies is complete ${ }^{l}$ and subsequent investigations will attempt to confirm the collectivity in the continuum through measurements of lifetimes and feeding times.

* Purdue University, West Lafayette, Indiana.

† Chemistry Division, ANL.

${ }^{1}$ P. Chowdhury et al., Phys. Rev. Lett. 47, 778 (1981).

d. Lifetime of Continuum States in ${ }^{154} \mathrm{Er}$

P. Chowdhury, I. Ahmad, ${ }^{*}$ Y. H. Chung, ${ }^{\dagger}$ P. J. Daly, ${ }^{\dagger}$ S. R. Faber, ${ }^{\dagger} Z$. Grabowsk1, T. L. Khoo, A. Pakkanen, $\dagger$ and D. Ward $\neq$

The yrast states of ${ }^{152}$ Dy are predominantly of aligned-particle nature up to the highest spin (40) observed. However, from examining the yrast population pattern and the continuum $\gamma$ spectra, we have previously found evidence suggesting the occurrence of collective structures above the

*Chemistry Division, ANL.

${ }^{\dagger}$ Purdue University, West Lafayette, Indiana.

${ }^{\ddagger}$ Chalk River Nuclear Laboratories, Chalk River, Ontario, Canada. 
yrast line. Lifetime measurements should provide direct confirmation of this suggestion.

We have employed a Doppler shift attenuation method to measure the lifetimes of continuum states in ${ }^{154} \mathrm{Er}$, an isotone of ${ }^{152} \mathrm{Dy}$. With the $\left({ }^{64} \mathrm{Ni}, 4 \mathrm{n}\right)$ reaction, evaporation-residue recoil velocities of $\sim 4 \%$ were possible. By comparing the continuum spectra from residues recoiling into vacuum and from residues stopping in $\mathrm{Au}$ or $\mathrm{Pb}$ foils, it should be possible to obtain a measure of the continuum-state lifetimes. In these measurements we employed $25 \times 30 \mathrm{~cm} \mathrm{NaI}$ crystals with $10 \mathrm{~cm}$ diameter central collimators. The photomultiplier gain was kept constant to better than $0.25 \%$ by monitoring the output from a stabilized LED.

Analysis of the data is in progress. One unexpected result so far is the observation that the statistical $\gamma$ rays $\left(\mathrm{E}_{\gamma}>2 \mathrm{MeV}\right)$ have long $(\geq 2 \mathrm{ps})$ decay times. These have hitherto been assumed to occur in the earliest stages of $r$ deexcitation. The reason for the observed retardation is not yet clear. The delayed statistical $\gamma$ rays in ${ }^{154}$ Er may be associated with the final stages of decay into the yrast region, following the collective cascades, as the collectivity is reduced towards the aligned-particle yrast line. We plan to survey the statistical lifetimes for different types (single-particle, deformed, transitional) of nuclei. In addition, we intend to measure the feeding times of the highest spin states using our recently constructed plunger. The angular momentum dependence of this feeding time, as can be inferred from measurements with a sum spectrometer, will provide a rather direct probe of the structure of continuum states.

e. The $\left(\mathrm{h}_{11 / 2}\right)^{4}$ Spectrum in the Four Valence Proton Nucleus ${ }^{150} 60^{*} \mathrm{Er}_{82}$

Y. H. Chung, ${ }^{*}$ H. Helppi, ${ }^{*}$ I. Ahmad, ${ }^{\dagger}$ P. Chowdhury, P. J. Daly, ${ }^{*}$ S. R. Faber, * Z. Grabowsk1, R. V. F. Janssens, T. L. Khoo, A. Pakkanen, * R. D. Lawson, and J. Blomqvist申

The nucleus ${ }_{64}^{146} \mathrm{Gd}_{82}$ shows many properties of a doubly-closed shell nucleus and suggests a sizable gap in the single-particle proton spectra at $Z=64$. We have systematically investigated $N=82$ nuclei with several "valence" protons beyond $Z=64$ in order to determine whether this may be

\footnotetext{
* Purdue University, West Lafayette, Indiana.

${ }^{\dagger}$ Chemistry Division, ANL.

${ }^{\psi^{*}}$ Research Institute of Stockholm, Sweden.
} 


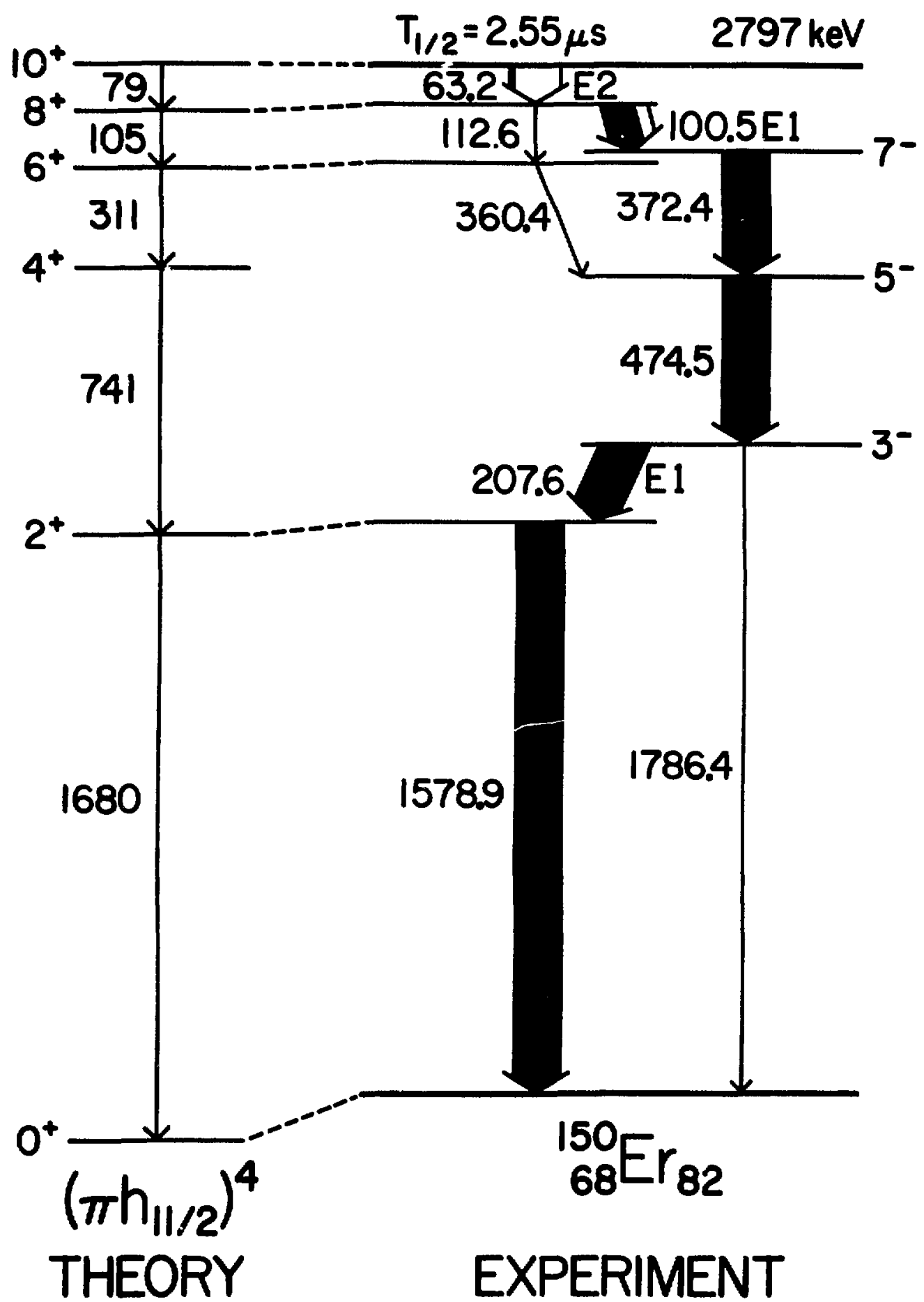

Fig. II-2. Experimental ${ }^{150}$ Er level scheme up to the $2.55 \mu \mathrm{sec}$ isomer, and calculated $\left(\pi h_{11 / 2}\right)^{4}$ yrast levels. 
used as a core in shell-model calculations of high-spin yrast states. Our earlier studies of the 3 valence proton nucleus ${ }_{67}^{149}{ }^{\mathrm{Ho}} 82$ showed very good agreement with theory for both the level structure and the $27 / 2^{-}+23 / 2^{-}$E2-transition lifetime. 1

${ }^{58} \mathrm{Ni}$ - and ${ }^{60} \mathrm{Ni}$-induced reactions on several targets were used to produce ${ }^{150} \mathrm{Er}$, the level scheme of which was completely unknown. The target was located in a NaI sum spectrometer and the evapcration residues were allowed to recoil out to a catcher foil. The activity on this foil was observed with $\mathrm{Ge}$ and $\mathrm{NaI}$ detectors in delayed coincidence with the sum energy signal. Isotopic identification came from the characteristic $x$ rays (which give $Z$ ), the sum energy (which gives $A$ ), and cross-bombardment results. A $2.6 \mathrm{\mu s} 10^{+}$isomer was identified, in addition to several higher lying isomers with spin larger than 218 .

The yrast levels to spin 16 showed very good agreement with the calculated $\left(h_{11 / 2}\right)^{4}$ spectrum (see Fig. II-2); furthermore, the $B(E 2)$ for the $10^{+} \rightarrow 8^{+}$transition is also in excellent agreement with theory. Analysis of the data on the higher lying isomers is continuing.

${ }^{1}$ J. Wilson et al., Z, Phys. A296, 185 (1980). f. The $\left(h_{11 / 2}\right)^{5}$ spectrum in ${ }^{151} \operatorname{Tm} 82$ Y. H. Chung, ${ }^{*}$ H. Helppi, I. Ahmad, ${ }^{\dagger}$ P. Chowdhury, P. J. Daly, ${ }^{*}$ S. R. Faber, ${ }^{*}$, Grabowski, T. I. Khoo, A. Pakkanen, * R. D. Lawson, and J. BlomquistF

This study is a continuation of the program described in the preceding contribution. A $470 \mathrm{n} \approx 27 / 2^{-}$isomer has been found and the level structure below the isomer is weil described by a seniority $3,\left(h_{11 / 2}\right)^{5}$ configuration. In addition, the half-1ife is in good agreement with theory. Thus shell-model calculations assuming a $Z=64, \mathrm{~N}=82$ core [with matrix elements and an effective charge derived from the $\left(h_{11 / 2}\right)^{2}$ spectrum] can accurately predict the energy levels and lifetimes for the $\left(h_{11 / 2}\right)^{n}$ configuration, where $n=3-5$.

The $N=82$ nuclei investigated in this program are very neutron deficient and are, in fact, close to the proton-drip line. Such

\footnotetext{
*Purdue University, West Lafayette, Indiana.

${ }^{+}$Chemistry Division, ANL.

íResearch Institute of Stockholm, Sweden.
} 
spectroscopic studies on nuclei so far from stability have been made feasible by the combination of several factors. First, the use of 58,60 Ni-induced reactions makes it possible to produce compound nuclei at low excitation energy, thereby limiting the number of final systems produced. Second, the use of the sum spectrometer, together with recoil-catcher techniques, provides very clean spectra. This work also represents a close collaboration between the experimental and theoretical effort at Argonne.

Our next goal is to produce the 6 valence proton nucleus ${ }^{152} \mathrm{Yb}$. A long-lived isomer is predicted here and the beam deflector plates, which will soon be installed at the linac, will greatly facilitate this study.

g. High Spin States in ${ }^{186} \mathrm{Hg}$

R. V. F. Janssens, P. Chowdhury, T. L. Khoo, Y. H. Chung, ${ }^{*}$ P. J. Daly, ${ }^{*}$

Z. Grabowski, ${ }^{*}$ M. Kortelahti, ${ }^{*}$ and J. McNeill ${ }^{*}$

The study of nuclear shapes along the yrast line at different spins and excitation energies is a topic of much current interest. Our recent investigations at the low-mass border $(A \simeq 150-160)$ of the rare earth region have shown a gradual change in character of the yrast states from ${ }^{148}$ Dy to ${ }^{158} \mathrm{Dy}$, as discussed above. At the high-mass border of this region $(A \simeq 180-190)$, the Hg isotopes also show a remarkable behavior. At low spin $\left(I \simeq 4^{+}\right)$a crossing occurs between a ground band with states equally spaced in energy and another band where energy spacings follow the $I(I+1)$ rotational rule. The current interpretation points towards a possible shape coexistence in these nuclei even though other models have been proposed. In fact, basic information about the sign of the ground state deformation and the behavior at high spin is unknown. Our investigation of ${ }^{186} \mathrm{Hg}$ with the ${ }^{156} \mathrm{Gd}\left({ }^{34} \mathrm{~S}, 4 \mathrm{n}\right)$ reaction is currently under way. In a first step, the deexcitation of discrete states is studied with standard gamma-ray techniques. The analysis is in progress. The known states (i.e., up to $12^{+}$) are clearly observed and new, not yet identified transitions are measured. From the multiplicity and energy information obtained from our sum spectrometer, it is clear that fission is a strongly competing reaction channel. This program has just started and we plan to extend the studies to neighboring nuclei. The properties of the $\gamma$-ray quasicontinuum will be studied. Indications for

* Purdue University, West Lafayette, Indiana. 
long-living isomers $\left(T_{1 / 2} \simeq 100 \mu s\right)$ exist and their decay will then be examined. It is also hoped to gain further insight in the competition between evaporation residue formation and fission.

h. Yrast Population Patterns in a Wide Range of Nuclei

J. Borggreen, * G. Sletten, , R. V. F. Janssens, I. Ahmad, ${ }^{\dagger}$ P. Chowdhury, T. L. Khoo, Y. H. Chung, $\neq$ P. J. Daly, $\neq$ Z. Grabowski, $\neq$ M. Kortelahti, $\neq$ and J. McNeilif

The purpose of this experiment is to investigate the dependence on nuclear structure of the relative population of states on the yrast line. In order to do this properly, the various nuclei under investigation should all be produced with $(\mathrm{HI}, \mathrm{xn})$ reactions bringing in similar angular momenta and excitation energies. We have studied the population pattern as a function of spin in the nuclei ${ }^{148 \rightarrow 156} \mathrm{Dy},{ }^{154 \rightarrow 160} \mathrm{Er},{ }^{146,147} \mathrm{Gd}$, populated in ${ }^{34} \mathrm{~S}$ and ${ }^{30} \mathrm{Si}$ induced reactions. The setup involved: (i) a Ge(Li) detector located at $55^{\circ}$ with respect to the beam axis, (ii) a sum spectrometer used either for the study of the total $\gamma$ energy emitted or for the investigation of the $\gamma$ multiplicity, and (iii) two $\mathrm{NaI}\left(\mathrm{T} 1\right.$ ) detectors $10 \mathrm{cated}$ at $90^{\circ}$ and $0^{\circ}$, respectively, to measure the "continuum" spectra. The analysis is currently in progress. Striking differences have been observed between the feeding patterns in the light and heavy Dy isotopes.

*Niels Bohr Institute, Copenhagen, Denmark.

${ }^{\dagger}$ Chemistry Division, ANL.

$\neq_{\text {Purdue University, West Lafayette, Indiana. }}$ 


\section{B. FUSION CROSS SECTIONS}

Over the past several years detailed studies of the fusion behavior of a large number of target-projectile combinations have been made at the tandem and more recently at the linac booster. These studies have been continued to some degree in the lighter systems, but in particular in the heavier systems since sufficiently high-energy ions up to $A \approx 80$ are now available from the linac to surmount the Coulomb barrier of even the heaviest systems. The effects of the structure of the target or projectile which have been observed in the lighter systems have been searched for in the heavier systems, with particular emphasis on exploration of pathways to the production of nuclei far off stability. New techniques, as the measurement of compound nuclear $x$ rays and the use of an electrostatic deflector to separate evaporation residues at $0^{\circ}$ from the beam particles, have been employed successfully. In the very heavy systems, where probabilities for compound nucleus formation and survival are extremely low, cross-section measurements in the micro- and nano-barn region are now feasible.

a. A Search for Structure in the Fusion of ${ }^{16} \mathrm{O}+{ }^{24} \mathrm{Mg}$

R. A. Racca, ${ }^{*}$ F. W. Prosser, ${ }^{*}$ C. N. Davids, and D. G. Kovar

Resonance-like structures have been seen in the excitation functions for fusion in light heavy-ion induced reactions involving " $\alpha$ nuclei" (e.g., ${ }^{12} \mathrm{C}$ and ${ }^{16} \mathrm{O}$ ), with spacings of about $5 \mathrm{MeV}$ and widths of about $2 \mathrm{MeV} .^{1}$ They have been reported in systems as heavy as ${ }^{12} \mathrm{C}+{ }^{24} \mathrm{Mg}$, ${ }^{2}$ but were not observed in somewhat heavier systems such as ${ }^{16} \mathrm{O}+{ }^{28} \mathrm{Si},{ }^{3}$ for example. We have searched for similar structures in the ${ }^{16} \mathrm{O}+{ }^{24} \mathrm{Mg}$ system, looking at the $y$-ray yields of transitions associated with evaporation residues in $350-\mathrm{keV}$ energy steps over the energy range $40 \mathrm{MeV} \leqslant \mathrm{E}_{1 \mathrm{ab}}\left({ }^{16} \mathrm{C}\right) \leqslant 51 \mathrm{MeV}$. While there is evidence for weak structure in the yields of some residues, cross correlation analysis indicates that no correlated structures exist at the statistical accuracy of the present data $(\leqslant 5 \%)$. This can be seen in Fig. II-3, where the cross correlation between $\gamma$-ray yields for transitions in ${ }^{35} \mathrm{Cl},{ }^{37} \mathrm{Ar}$, and ${ }^{38} \mathrm{Ar}$, which showed indications of "structure," displays no evidence of a correlated behavior as function of bombarding energy. Hence for ${ }^{12} \mathrm{C}$ and ${ }^{16} \mathrm{O}$ induced reactions, structure in the fusion channel is

*University of Kansas, Lawrence, Kansas.

${ }^{1}$ D. G. Kovar et al., Phys. Rev. C 20, 1305 (1979).

${ }^{2}$ K. Daneshvar et al., Phys. Rev. C 25, 1342 (1982).

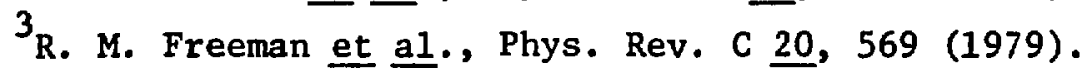




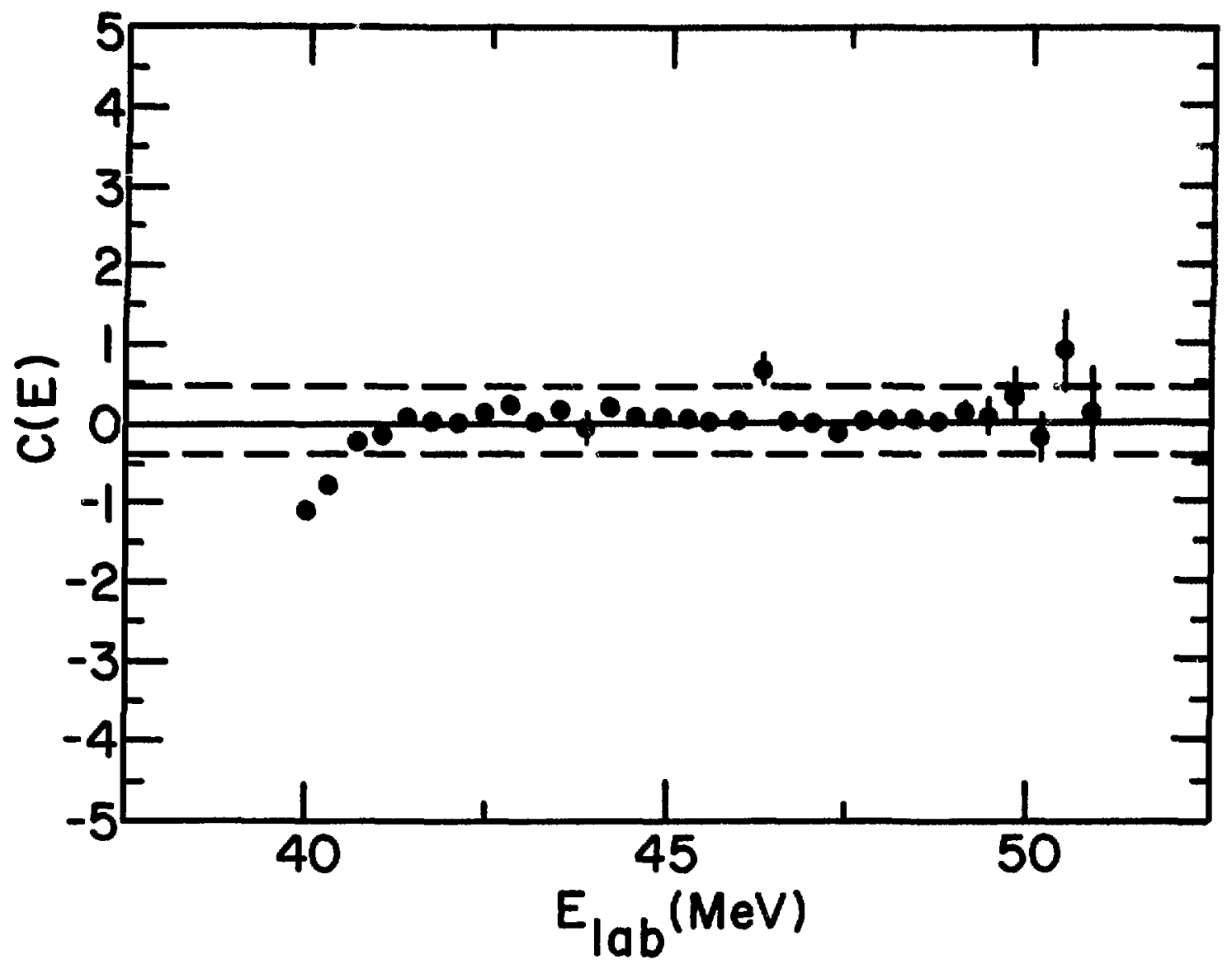

Fig. II-3. Cross correlation between the $\gamma$-ray transitions ${ }^{38} \mathrm{Ar}(669)$, $38 \mathrm{Ar}(2167), 37 \mathrm{Ar}(1611)$, and $35 \mathrm{Cl}(3162)$. An averaging interval of $5.25 \mathrm{MeV}$ was used. The estimated standard deviation in the correlation function based on $\operatorname{Ref}, 3$ is indicated by the horizontal dashed lines.

apparently limited to the systems ${ }^{12} \mathrm{C}+{ }^{24} \mathrm{Mg}$ and 1 ighter, and ${ }^{16} \mathrm{O}+{ }^{20} \mathrm{Ne}$ and lighter, even though structure in the elastic, inelastic, and transfer reaction channels continues to be observed for heavier systems (e.g., ${ }^{12} \mathrm{C}+{ }^{28} \mathrm{Si},{ }^{32} \mathrm{~s}$ and $\left.{ }^{16} \mathrm{O}+{ }^{24} \mathrm{Mg},{ }^{28} \mathrm{Si},{ }^{32} \mathrm{~s}\right) \cdot{ }^{4,5}$ This behavior would appear to be a significant feature to be reproduced by any reaction model which hopes to explain the resonance phenomena; at present no such model exists.

${ }^{4} \mathrm{~J}$. Barrette et al., Phys. Rev. C 20, 1959 (1979).

${ }^{5}$ S. J. Sanders et al., Phys. Rev. C 21 , 1810 (1980). 


\section{b. Influence of the Entrance Channel on the Fusion Process}

R. A. Racca, ${ }^{\star}$ F. W. Prosser, Jr., ${ }^{\star}$ C. N. Davids, and D. G. Kovar

In charged-particle measurements of the total fusion cross-section behavior at energies above the Coulomb barrier for the systems ${ }^{15} \mathrm{~N}+{ }^{27} \mathrm{Al}$, ${ }^{16} \mathrm{O}+{ }^{26} \mathrm{Mg}$, and ${ }^{18} \mathrm{O}+{ }^{24} \mathrm{Mg}$ (which form the same compound nucleus ${ }^{42} \mathrm{Ca}$ ), evidence for an entrance channel effect was observed. ${ }^{1}$ In the present study, $\gamma$-ray measurements were performed for the ${ }^{16} \mathrm{O}+{ }^{26} \mathrm{Mg}[25 \mathrm{MeV}$ $\left.\leqslant E_{1 a b}\left({ }^{16} 0\right) \leqslant 70 \mathrm{MeV}\right]$ and ${ }^{18} 0+{ }^{24} \mathrm{Mg}\left[27 \mathrm{MeV} \leqslant \mathrm{E}_{1 \mathrm{ab}}\left({ }^{18} 0\right) \leqslant 65 \mathrm{MeV}\right]$ systems in order to see whether the entrance channel effects would manifest themselves in the distribution of strength among the various evaporation nuclei produced. At lower bombarding energies, the results show that for the $160+{ }^{26} \mathrm{Mg}$ system, the residues with mass numbers 40 and 37 dominate, while for the ${ }^{18} \mathrm{O}+{ }^{24} \mathrm{Mg}$ system, the decay is dominated by fusion residues with mass numbers 39 and 36 . Calculations with the statistical model code CASCADE $^{2}$ reproduce these behaviors, as well as the basic features of the residue distribution as function of bombarding energy; however, for both systems the predictions tend to underestimate the strengths observed for the heaviest residues (e.g., $\mathrm{m}=40$ for ${ }^{16} \mathrm{o}+{ }^{26} \mathrm{Mg}$ and $\mathrm{m}=39$ for ${ }^{18} \mathrm{o}+{ }^{24} \mathrm{Mg}$ ) and overestimate the strengths for mass $=36$. An example of the type of agreement that is obtained is shown in Fig. II-4, where the results for the $16 \mathrm{O}+{ }^{26} \mathrm{Mg}$ and ${ }^{18} \mathrm{O}+{ }^{24} \mathrm{Mg}$ reactions forming the compound system ${ }^{42} \mathrm{Ca}$ at an excitation of $\mathrm{E}_{\mathrm{x}}=48.5 \mathrm{MeV}$ are compared with the predictions of CASCADE. These discrepancies are most pronounced at the lower bombarding energies, where the fusion process is dominated by the interaction barrier. Further work is necessary to determine whether these discrepancies are indications of shortcomings of the model calculations or of entrance channel effects.

\footnotetext{
*University of Kansas, Lawrence, Kansas.

${ }^{1}$ F. W. Prosser, Jr. et al., Phys. Rev, C 21,1819 (1980).

2 F. Puhlhofer, Nucl. Phys. A280, 267 (1977).
} 


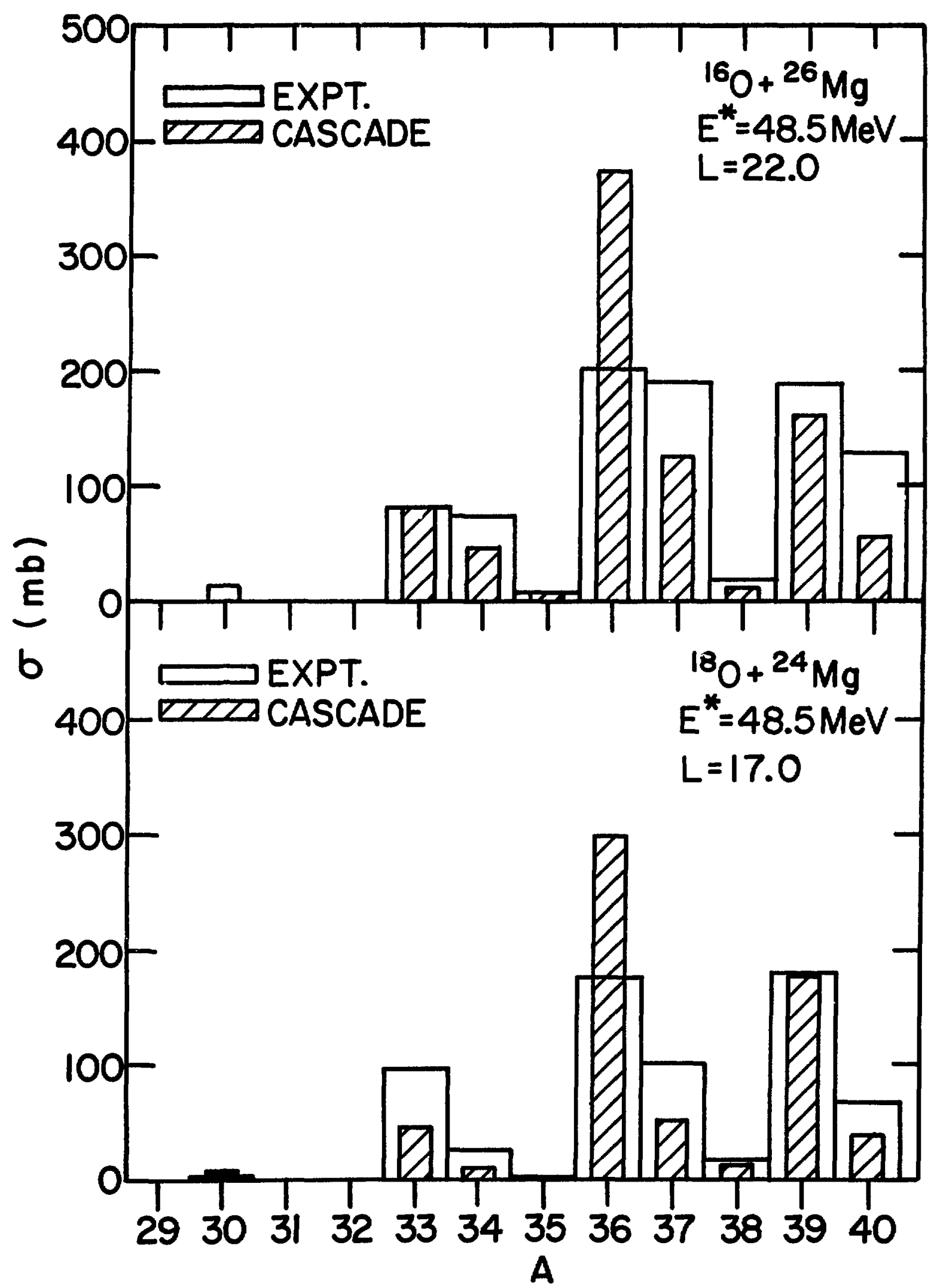

F1g. II-4. Mass number plots for the ${ }^{18} \mathrm{O}+{ }^{24} \mathrm{Mg}$ and ${ }^{16} \mathrm{O}+{ }^{26} \mathrm{Mg}$ systems at an excitation energy of $48.5 \mathrm{MeV}$. The shaded rectangles are the predictions of CASCADE. 


\section{c. Prompt Compound Nuclear $\mathrm{K} x$ Rays in Fusion Reactions Induced by a Heavy Projectile}

H. Ernst, W. Henning, C. N. Davids, W. S. Freeman, T. J. Humanic, M. Paul, * and S. J. Sanders ${ }^{\dagger}$

The limits of complete fusion and compound nucleus formation in a nuclear reaction induced by a heavy projectile are currently of great interest. In particular, increases in fusion threshold energies, as predicted in dynamical fusion-model calculations, will limit compound nucleus formation in heavy systems. An unambiguous signature of complete fusion is the observation of compound nucleus residues after light particle evaporation. An identification is in principle possible, even for very short-lived compound nuclei, through the detection of prompt characteristic $x$ rays, originating mainly from converted electromagnetic transitions in the evaporation residues, with $\mathrm{K} x$-ray lifetimes, for example, as short as $10^{-15}$ to $10^{-17} \mathrm{sec}$ in heavy systems.

We have studied prompt $K \times$ rays in compound nuclear reactions induced by a heavy projectile, by determining total production cross sections and multiplicities of $\mathrm{K} \times$ rays for the systems ${ }^{32} \mathrm{~s}+116,120,124 \mathrm{Sn}$ from $\mathrm{x}$-ray singles, $x$-ray-x-ray coincidences, and direct evaporation residue yields over the incident energy range $130 \mathrm{MeV} \leqslant E_{1 a b} \leqslant 202 \mathrm{MeV}$. We find a slow dependence of the multiplicities (Fig. II-5) and cross sections on target mass and incident energy, establishing the prompt $\mathrm{K} x$-ray yields as a reliable indicator of the complete fusion cross-section behavior induced by a heavy projectile in this mass region.

*Hebrew University, Jerusalem, Israel.

${ }^{\dagger}$ Yale University, New Haven, Connecticut.

\section{d. Fusion, Fission, and Deep Inelastic Reaction Cross Sections for}

$32 \mathrm{~s}+112,116,120,124 \mathrm{sn}$

H. Ernst, C. N. Davids, W. S. Freeman, W. Henning, T. J. Humanic, F. W. Prosser, * and R. Racca*

In extension of our complete fusion studies of the reactions ${ }^{32} \mathrm{~s}+\mathrm{sn}$ using $\mathrm{x}$-ray techniques, we have measured excitation functions for directly detected evaporation residues, and at one incident energy (247 MeV) the

\footnotetext{
*University of Kansas, Lawrence, Kansas.
} 


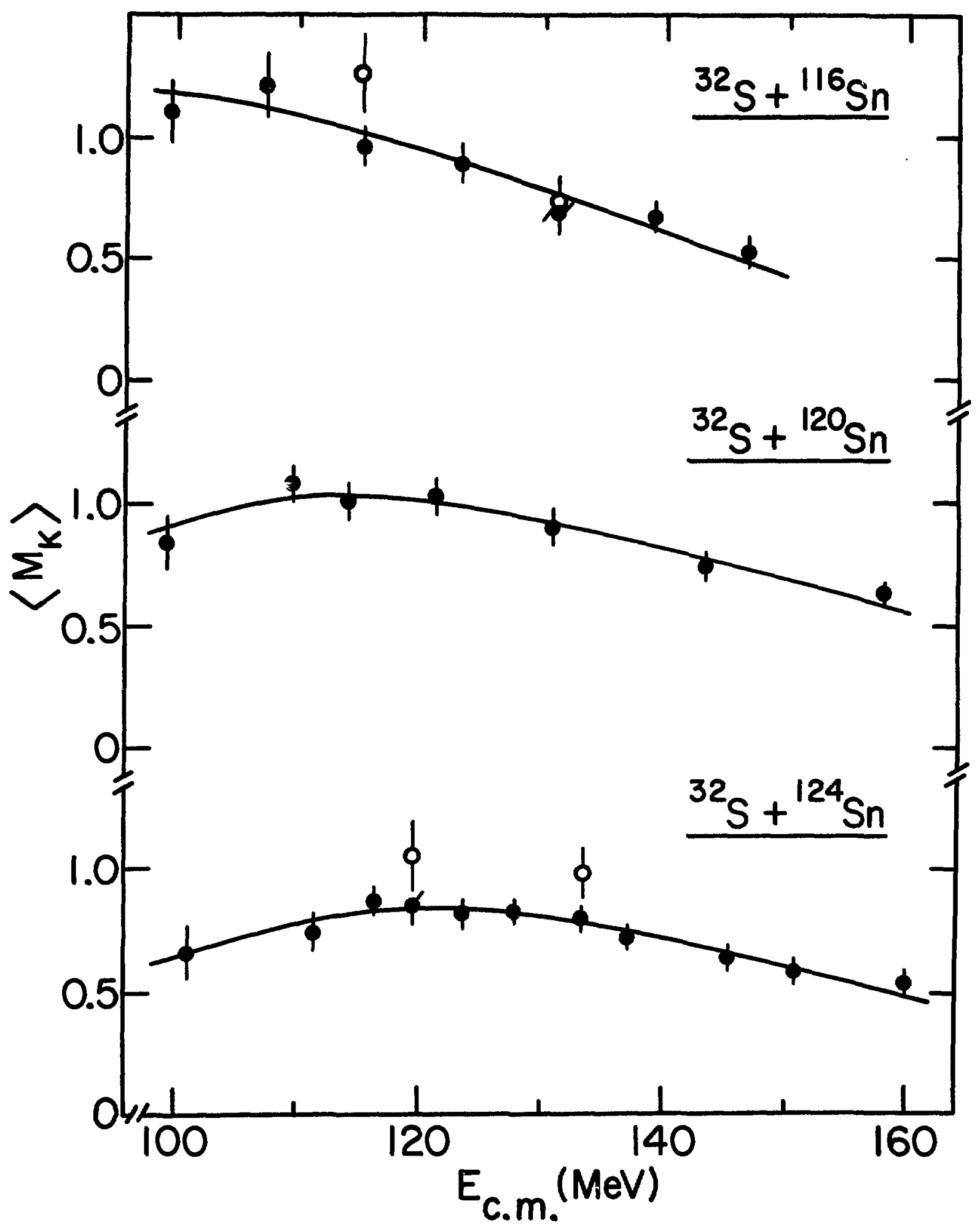

Fig. II-5. Total $\mathrm{X}$ x-ray multiplicttles $\left\langle M_{K}\right\rangle$ for ${ }^{32} S+116,120,124$ sn versus center-of-mass energy, as obtained from the measured $K$-vacancy production and evaporation residue cross sections (full dots). The values of $\left\langle M_{K}\right\rangle$ derived from $x$-ray- $x$-ray colncidence measurements are Indicated as open circles. The smooth lines are drawn to guide the eye. 
fusion-fission cross sections and the total reaction cross sections for ${ }^{32} \mathrm{~S}+112,116,120,124 \mathrm{Sn}$. Energy spectra and angular distributions of fission fragments and quasielastic and deep inelastic reaction products were measured with a large position-sensitive gas detector that was developed recently at Argonne. The position signal is derived from a single wire using the charge-division method, two energy-loss signals are provided by a split anode, and the total-energy signal is taken from the cathode. The derived reaction cross sections of $\sim 2800 \mathrm{mb}$ agree with optical-model calculations based on the elastic scattering cross sections. The total compound nucleus formation cross sections, i.e., evaporation residue plus fission yields, are $21100 \mathrm{mb}$ for all isotopes, while the evaporation residue cross sections alone show distinct differences for the various targets and range in maximum value between $55 \mathrm{v}$ and $800 \mathrm{mb}$ (Fig. II-6).

e. Fusion of ${ }^{58} \mathrm{Ni}+114,116,118,120,122,124 \mathrm{Sn}$

W. S. Freeman, H. Ernst, D. F. Geesaman, W. Henning, T. J. Humanic, W. Kühn, F. W. Prosser,* J. P. Schiffer, and B. Zeidman

Detailed measurements of the evaporation residue yields following fusion reactions for the ${ }^{58} \mathrm{Ni}+\mathrm{Sn}$ system have been obtained. Excitation functions for ${ }^{58} \mathrm{Ni}$ ions incident on the even-Sn isotopes were measured in the energy range $230<\mathrm{E}_{1 \mathrm{ab}}<322 \mathrm{MeV}$ (see Fig. II-7). An electrostatic deflector placed after the target was used to separate the evaporation residues emerging from the target at $\theta_{1 a b}=0^{\circ}$ from the intense flux of beam particles. We find that the maximum yield for all targets is near $180 \mathrm{MeV}$ (c.m.) with an increase in the cross section at this maximum of about a factor of four as one goes from ${ }^{114} \mathrm{Sn}$ to ${ }^{124} \mathrm{Sn}$. These measurements complement previous fusion-fission data for the same system and bear directly on the question of compound nucleus survivability as one goes to collisions between heavier ions. Related topics of current interest include possible entrance channel or shell effects, and whether or not an "extra push" is needed to achieve fusion in such heavy systems. We plan to extend these measurements, using a ${ }^{64} \mathrm{Ni}$ beam to populate some of the same compound nuclei at similar excitation energies and spins. In addition, the measurements will be extended to heavier targets, utillzing a deflector-detector geometry of increased efficiency.

\footnotetext{
*University of Kansas, Lawrence, Kansas.
} 


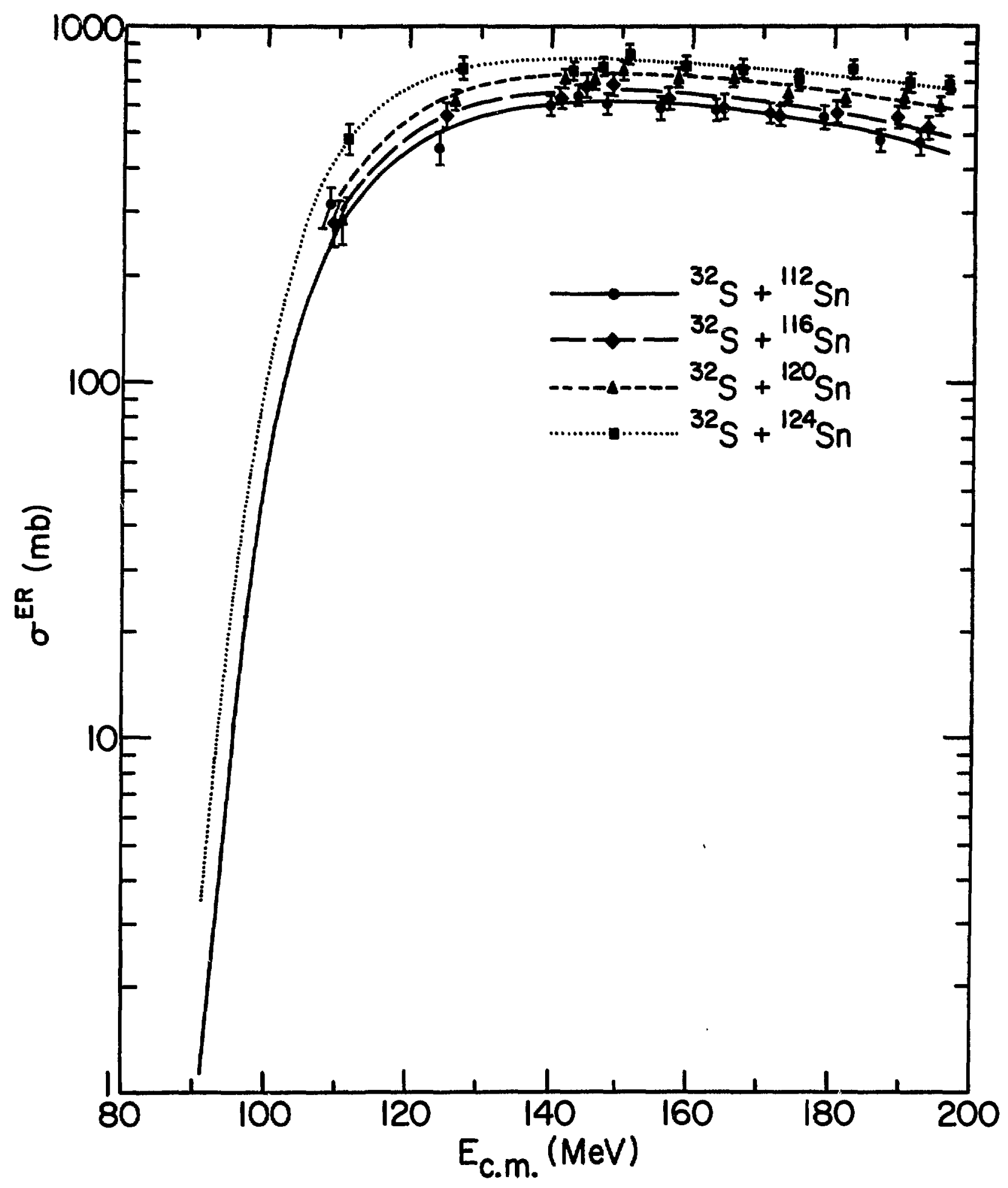

FIg. II-6. Evaporation residue cross sections from a direct measurement of the recolling residues with a $\Delta E-E$ particle telescope. 


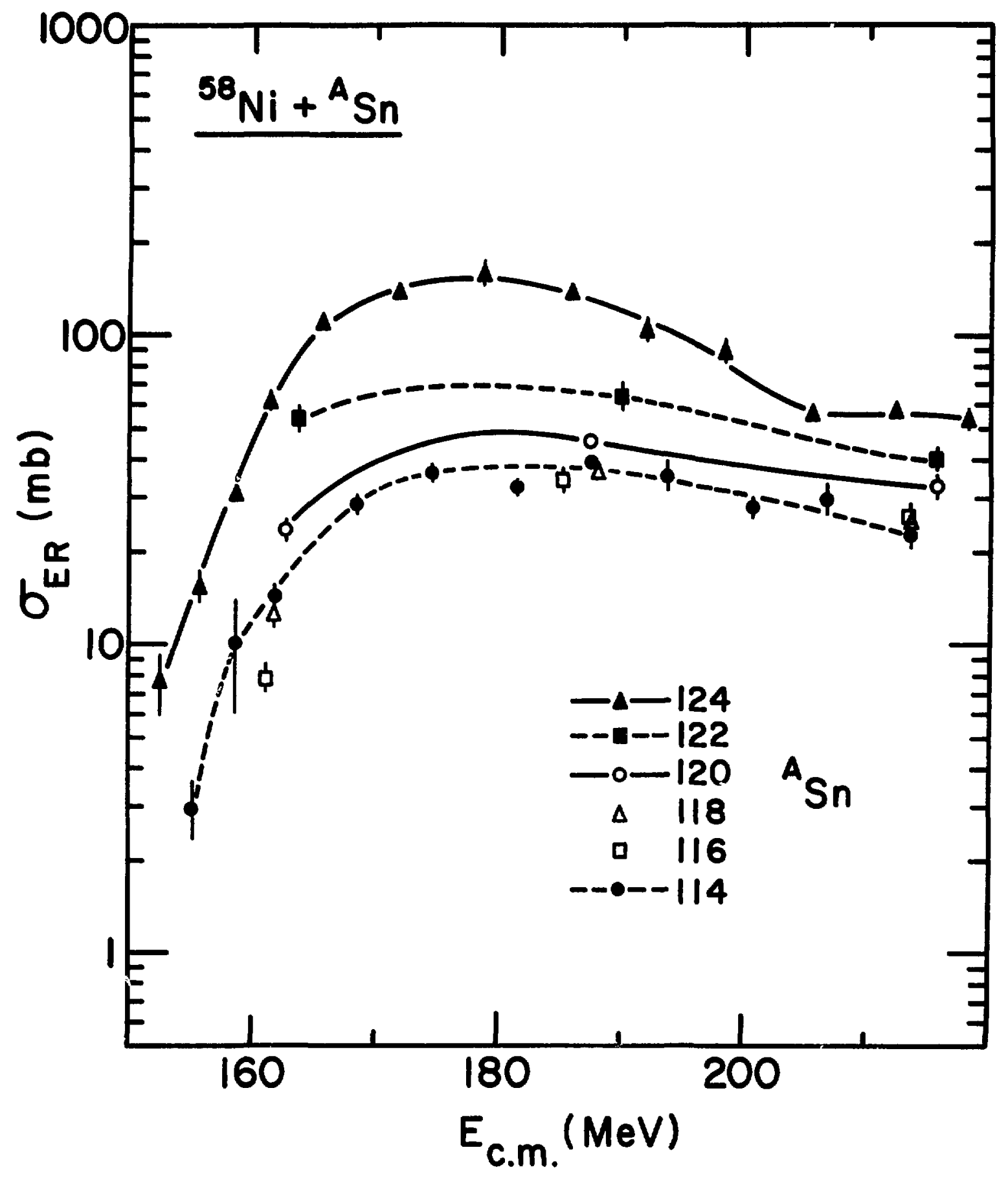

Fig. II-7. Evaporation-residue (ER) cross sections for ${ }^{58} \mathrm{Ni}$ bombarding the even-mass $\mathrm{Sn}$ isotopes $(A=114-124)$ as a function of center-ofmass energy. The lines are drawn only to guide the eye. 


\section{REACTION MECHANISMS AND DISTRIBUTION OF REACTION STRENGTHS}

The studies of reaction mechanisms and distribution of reaction strengths have been extended to higher energies, heavier projectiles (up to $A=60$ ) and heavier target nuclei as a consequence of increased accelerator capability. Transitions to discrete final states, which can be treated in a microscopic model, as well as the general distribution of reaction flux, have been studied in ${ }^{16} 0$-induced collisions. The aim of these studies is to gain an understanding of the transition from purely quasielastic to the strongly damped processes. Velocity spectra to separate complete from incomplete fusion processes, light particle coincidences to investigate final state interactions, neutron spectra and characteristic $\gamma$ rays from quasielastic and deep-inelastic fragments to obtain high-resolution information on these processes, have been studied and open new perspectives in the study of reaction mechanisms.

a. Time-of-Flight Measurements of Evaporation Residues Produced in ${ }^{16} \mathrm{O}+{ }^{12} \mathrm{C}$ and $160+24 \mathrm{Mg}$ Reactions at $4 \leqslant \mathrm{E}_{1 \mathrm{ab}}(160) \leqslant 9.5 \mathrm{MeV} /$ Nucleon

T. J. Humanic, D. G. Kovar, R. R. Betts, * P. Chowdhury, D. Henderson,

R. V. F. Janssens, W. Kühn, and K. L. Wolf*

In order to understand the reaction mechanism for heavy-ion induced reactions at higher bombarding energies ( $E_{1 a b}>5 \mathrm{MeV} /$ nucleon), it is important to be able to distinguish between complete fusion and incomplete fusion (where only a portion of the projectile fuses with the target) processes. It has been shown that the shapes and centroids of the velocity spectra of evaporation residues produced in heavy-ion reactions can be used to determine whether the products originated from the projectile plus target compound nucleus, i.e., whether full momentum transfer has occurred. Velocity spectra showing deviations from that predicted for complete fusion are indicative of contributions from incomplete fusion processes and provide a measure of the relative importance of such processes. The ${ }^{16} \mathrm{O}+{ }^{12} \mathrm{C}$ and $16 \mathrm{O}+{ }^{24} \mathrm{Mg}$ systems have been studied over the energy range $4 \leqslant \mathrm{E}_{1 \mathrm{ab}}\left({ }^{16} 0\right) \leqslant 9.5$ $\mathrm{MeV} /$ nucleon (Fig. II-8). Measurements were performed using a time-of-flight detection system whose timing resolution $\left(\Delta t_{F W H M} \approx 130\right.$ picoseconds) makes possible the extraction of velocity spectra for the individually resolved evaporation residue masses. Analyses of these results are underway and plans have been made for coincldence measurements between the evaporation residue-like products and the forward-peaked energetic 1ight particles (e.g., $p, d, \alpha)$ in order to further investigate the incomplete fusion mechanism.

*Chemistry Division, ANL. 

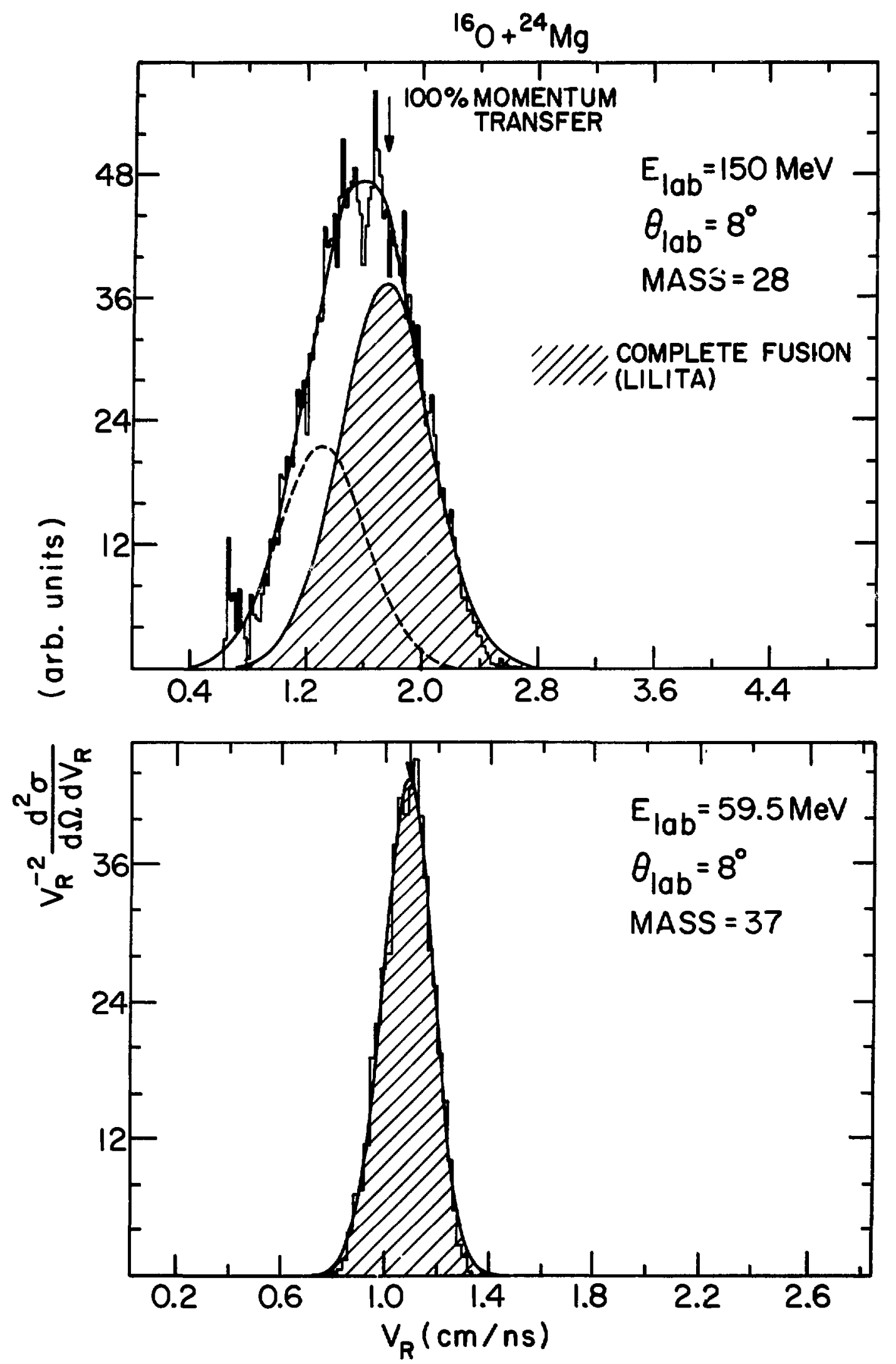

Fig. II-8. Velocity spectra of evaporation residue-like fragments for $160+24 \mathrm{Mg}$. 
b. Observation of Final State Interaction After Heavy-Ion-Induced Fusion Reactions

W. Kühn, H. Ernst, W. S. Freeman, W. Henning, T. J. Humanic, and J. P. Schiffer

Light particle energy and angular correlations at backward angles as well as singles excitation functions and angular distributions have been measured in the system ${ }^{32} \mathrm{~S}+{ }^{40} \mathrm{Ca}$ in the incident energy range $\mathrm{E}_{1 \mathrm{ab}}=100$ $\mathrm{MeV}$ to $\mathrm{E}_{\mathrm{lab}}=250 \mathrm{MeV}$. A previous experiment had shown that the light particle coincidence cross section is affected by final state interaction between the outgoing light particles. The present experiment was set to observe this effect as a function of the compound nucleus excitation energy, thus varying the compound nucleus lifetime. Since the magnitude of the final state interaction is dependent on the spatial and temporal distance between the outgoing light particles, it should show a significant lifetime dependence. As a consequence, it might be possible to develop a new method for lifetime measurements in the unexplored range below $10^{-21} \mathrm{sec}$. We have measured $\alpha-\alpha$ and $p-p$ coincidences, and a preliminary analysis shows effects from final state interactions. Presently, the final data analysis is in progress.

c. AnguJ.ar Momentum Dependence of Neutron Spectra in the $250-\mathrm{MeV}{ }^{64} \mathrm{Ni}+{ }^{92} \mathrm{Zr}$ Reaction

W. Kühn, P. Chowdhury, R. V. F. Janssens, T. L. Khoo, F. Haas, * J. Kasagi,* and R. Ronningen*

We have started ar. experiment to investigate the properties of compound nuclei at relatively low excitation energy above the yrast line. In this region, the compound nucleus decays predominately by $2-n$ or $3-n$ emission, followed by a gamma cascade. Since there are not many other particle decay channels competing, the detection of the neutrons provides a probe for the early stages after compound nucleus formation. It may as well provide a clean and direct test of statistical model predictions.

Neutron spectra in coincidence with the gamma-ray sum energy have been measured in the $250-\mathrm{MeV}{ }^{64} \mathrm{Ni}+{ }^{92} \mathrm{Zr}$ reaction. We observe that the spectra coincident with higher gamma-sum energies, corresponding to the decay of higher angular momentum states, are characterized by lower temperatures. Presumably, this reflects the fact that the excitation energy above the

*Michigan State University, East Lansing, Michigan. 
compound nucleus yrast line decreases with angular momentum. We plan to compare the results with statistical model predictions.

We shall also explore the possibility of gating on the neutron spectrum to select nuclei produced at high spin but close to the yrast line, i.e., "cold" nuclei.

\section{d. Observation of Characteristic Gamma Rays from Quasielastic and Deep-} Inelastic Fragments in the $58 \mathrm{Ni}+58 \mathrm{Ni}$ Reaction

W. Kühn, R. V. F. Janssens, and T. L. Khoo

We have started an experiment to study the transition between quasielastic reactions, being strongly dependent on nuclear structure, and deep-inelastic reactions, being dominated by statistical features. We have measured coincidences between the gamma sum energy and characteristic gamma rays from one (2-fold coincidence) and both (3-fold coincidence) of the fragments from $345-\mathrm{MeV}{ }^{58} \mathrm{Ni}+{ }^{58} \mathrm{Ni}$.

First results from a preliminary data analysis allowed us to identify several discrete transitions in ${ }^{60} \mathrm{Ni},{ }^{59} \mathrm{Ni},{ }^{58} \mathrm{Ni},{ }^{57} \mathrm{Fe},{ }^{56} \mathrm{Fe}$, and ${ }^{54} \mathrm{Fe}$. By gating on different gamma sum energies, we can discriminate between quasielastic and deep-inelastic processes. We plan to extend our measurements to different target/projectile combinations. 


\section{RESONANT STRUOTURE IN HEAVY-ION REACTIONS}

In the past several years extensive studies exploring the strong resonance-like structures in $s-d$ shell nuclei have been performed. The ease with which beam energy can be varied on the linac has allowed studies begun at the tandem to be extended to higher energies. Over the last year selected measurements have been made to study specific questions; in addition, detailed analyses of previous data have been performed. The questions studied address resonant exchange between nearly degenerate channels, massive transfer to molecular resonance states, and an extension of the searches for structure in quasielastic channels to much heavier systems, with compound nucleus mass $A=80$.

a. Search for Resonant Exchange in the Reaction ${ }^{17} \mathrm{O}+{ }^{48} \mathrm{Ca}$

S. J. Sanders, * W. S. Freeman, W. Henning, T. J. Humanic, and W. Kühn

It has been suggested that the structures observed in excitation functions of quasielastic channels in heavy-ion reactions, extending well into the s-d shell, are connected to resonant particle or cluster exchange between nearly degenerate energy levels. To test this hypothesis, the reaction ${ }^{17} \mathrm{O}+{ }^{48} \mathrm{Ca}$ was studied at $45-\mathrm{MeV}$ incident energy. Considerations in a schematic two-center molecular orbital picture suggest ${ }^{1}$ that the inelastic scattering channel $170^{*}\left(1 / 2^{+}\right)$and the single neutron-transfer channel to the first excited state in ${ }^{49} \mathrm{Ca}\left(1 / 2^{-}\right)$are nearly degenerate in energy at an interaction distance around the strong absorption radius. This could lead to strong resonant exchange if that reaction mode exists. Our measurements do not reveal any such strong structure, with an upper 1 imit of $\leqslant 3 \%$ of the average cross section.

*Yale University, New Haven, Connecticut.

${ }^{1}$. J. Ascuitto (private communication, to be published).

b. Search for Massive Transfer to Molecular Resonance States in the Reaction $12 \mathrm{C}+160+24 \mathrm{Mg} \mathrm{g}^{*}+\alpha+12 \mathrm{C}+12 \mathrm{C}$ at $145 \mathrm{MeV}$

W. S. Freeman, D. Frekers, H. Ernst, W. Henning, W. Kühn, L. Bland,* H. T. Fortune,* P. Kutt,* G. Stephans,* R. Segel, ${ }^{+}$and S. Mukhopadhyay $\dagger$

The question whether the structures observed recently in $\alpha$-singles spectra at very negative $Q$ values in the reaction ${ }^{16} \mathrm{O}+{ }^{12} \mathrm{C}$, at incident

\footnotetext{
*University of Pennsylvania, Philadelphia, Pennsylvania.

${ }^{\dagger}$ Northwestern University, Evanston, Illinois.
} 
energies high above the barrier $\left[E_{1 a b}\left({ }^{16} 0\right)=145 \mathrm{MeV}\right]$, signify a massive ${ }^{12} \mathrm{C}$ transfer to molecular resonances in $24 \mathrm{Mg}$, is still not answered. In view of the importance of such a process, if verifled, we decided to perform a triple-coincidence measurement between outgoing $\alpha$ particles and the ${ }^{12} \mathrm{C}+{ }^{12} \mathrm{C}$ final channel. The $145-\mathrm{MeV}{ }^{16} \mathrm{O}$ beam was provided by the 1 inac, and reaction products were detected with an $\alpha$-particle telescope and two large-area heavy-ion detectors, one a silicc s surface barrier and one a gas counter. Singles $\alpha$ spectra and coincidence spectra were measured simultaneously. A total of 2600 triple coincidence events were recorded, which are presently being analyzed.

c. Elastic Scattering and Reactions of ${ }^{40} \mathrm{C} \bar{a}+{ }^{40} \mathrm{Ca}$ R. R. Betts, ${ }^{*}$ I. Ahmad, ${ }^{*}$ B. Back, ${ }^{*}$ B. G. Glagola, , W. Henning, S. Saini, and J. L. Yntema

We have extended our studies on resonant structures in excitation functions of quasielastic heavy-ion reactions to a much heavier system, ${ }^{40} \mathrm{Ca}+{ }^{40} \mathrm{Ca}$. If resonant structures can be observed in heavier systems, the ${ }^{40} \mathrm{Ca}+{ }^{40} \mathrm{Ca}$ system is a likely candidate because of the negative $Q$ values for most direct-reaction channels and the resulting surface transparency in the optical-model potential. The elastic scattering and reactions of ${ }^{40} \mathrm{Ca}+{ }^{40} \mathrm{Ca}$ were measured at laboratory energies from $130 \mathrm{MeV}$ to $240 \mathrm{MeV}$. The detector was placed at $\theta_{\mathrm{c}, \mathrm{m}}=90^{\circ}$ and subtended an angular range $77^{\circ}<\theta_{\text {c.m. }}<103^{\circ}$. We find that the elastic scattering cross section decreases smoothly with increasing energy over the entire range. At $\mathrm{E}_{\text {lab }} \approx 200$ $\mathrm{MeV}$ the cross section is $800 \mathrm{nb} / \mathrm{sr}$. The cross sections for quasielastic and deep inelastic scattering also vary smoothly with energy. We are presently analyzing the data and comparing to optical-model predictions (Fig. II-9), in particular to those with surface transparent potentials, which in the lighter systems predicted resonance-like structures.

${ }^{*}$ Chemistry Division, ANL. 


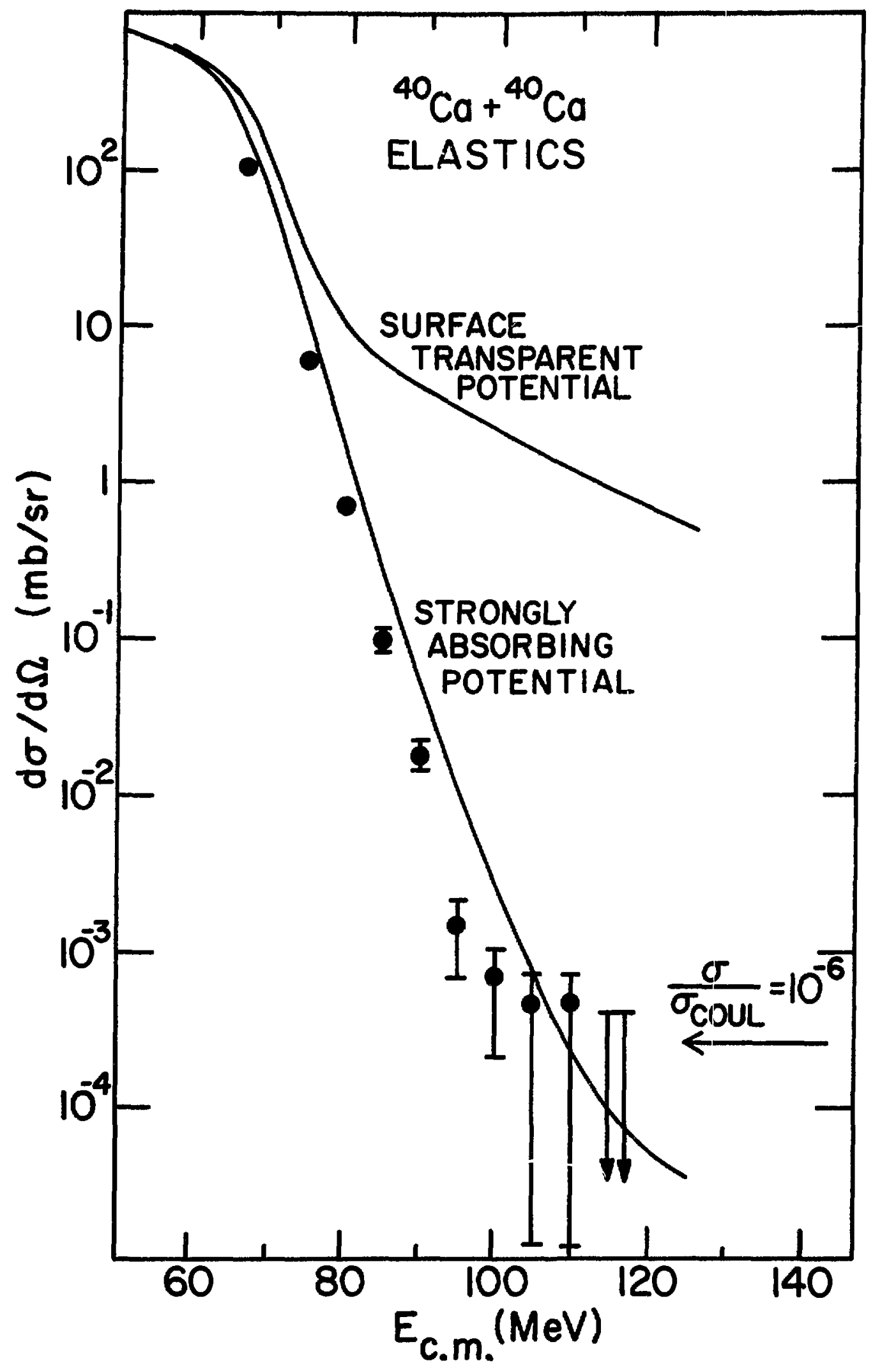

Fig. II-9. $90^{\circ}$ excitation function for ${ }^{40} \mathrm{Ca}+{ }^{40} \mathrm{Ca}$ elastic scattering. 


\section{E. ACCELERATOR MASS SPECTROMETRY}

Accelerator mass spectrometry has developed into an extremely highsensitivity tool for measurements of minute quantities of radioisotopes. A number of nuclear physics problems exist that at present can only be efficiently studied with the AMS technique, involving not only light nuclei generally studied in AMS, but also medium-heavy and heavy ones that now can be accelerated with the tandem-linac system. Nuclear cross sections of specific interest and lifetimes of isotopes of geophysical and cosmogenic interest are some of the examples studied at Argonne. The vitality of the field is demonstrated by an International Symposium on Accelerator Mass Spectrometry held at the Argonne Physics Division in May 1981, with representatives from essentially all laboratories now active in this new field.

\section{a. Symposium on Accelerator Mass Spectrometry at the ANL Physics Division, May 11-13, 1981}

W. Henning, W. Kutschera, R. K. Smither, and J. L. Yntema

A Symposium on Accelerator Mass Spectrometry (AMS) was organized at Argonne which gathered representatives from essentially all laboratories active in this new field. In attendance were 96 scientists of which 26 were from outside the United States. At present 17 laboratories are performing AMS experilrents on existing nuclear accelerators (almost exclusively tandems). Five dedicated facilities ( $3 \mathrm{MV}$ tandems) will become operational in 1982 in the USA, Canada, England, France, and Japan. The AMS technique to measure minute quantities of long-lived radioisotopes and its application in nuclear physics and interdisciplinary fields was discussed in more than 40 contributions. The proceedings were published as an ANL report (ANL/PHY-81-1) documenting progress and perspectives of this exciting new field.

\section{b. Cross Section of the ${ }^{27} \mathrm{Al}(\mathrm{n}, 2 \mathrm{n})^{26} \mathrm{Al}\left(\mathrm{T}_{1 / 2}=7.2 \times 10^{5} \mathrm{yr}\right)$ Reaction and Its Relevance to Displacement Damage Studies}

R. K. Smither, L. R. Grcenwood, W. Henning, W. Kutschera, and J. L. Yntema

The ${ }^{27} \mathrm{Al}(\mathrm{n}, 2 \mathrm{n}){ }^{26} \mathrm{Al}$ reaction contributes most of the displacement damage cross section in aluminum for fast neutrons with energies above 15 $\mathrm{MeV}$. The only other important reactions are elastic and inelastic neutron scattering with cross sections which are lower by a factor of $2-3$. The total cross section for $(n, 2 n)$ in aluminum has never been measured. Present damage calculations use a theoretically derived cross section for this 
reaction. Since this type of calculation can be in error by an unknown factor and the damage of fast neutrons is of considerable interest in connection with thermonuclear fusion studies, an experimental check of the cross section is important.

The AMS technique was used to measure the concentration of ${ }^{26} \mathrm{Al}$ in two sets of aluminum dosimetry foils that had been exposed to known doses of high-energy neutrons. ${ }^{26} \mathrm{Al} / /^{27} \mathrm{Al}$ ratios in the range from $10^{-8}$ to $10^{-12}$ were measured with the AMS system at the tandem, yielding cross sections in the 3 to $100 \mathrm{mb}$ range. The results indicate that the $(n, 2 n)$ cross section in the measured range from 14 to $24 \mathrm{MeV}$ is a factor of two lower than suggested by the theoretically calculated values that are presently being used in damage calculations.

\section{c. Measurement of the ${ }^{44}$ Ti Half-Life Via Tandem Accelerator Mass} Spectrometry

D. Frekers, W. Henning, W. Kutschera, K. E. Rehm, R. K. Smither, J. L. Yntema, and B. Stievano*

The half-life of ${ }^{44} \mathrm{Ti}$ has been measured from the decay rate and the radioisotope concentration. The latter quantity was measured using the Argonne $\mathrm{FN}$ tandem for high-sensitivity isotope detection. ${ }^{1}$ A natural $\mathrm{THO}_{2}$ sample, spiked with ${ }^{44} \mathrm{Ti}$ of about $1.2 \mu \mathrm{CI}$ total activity, was used as the source material. After stripping the injected $\mathrm{TiO}^{-}$beam in the terminal, ${ }^{44} \mathrm{Ti}^{9+}$ Ions were accelerated to $80 \mathrm{MeV}$ and Identified in an Enge split-pole magnetic spectrograph. The concentration of ${ }^{44} \mathrm{TI}$ in the source material was measured with respect to the ${ }^{46} \mathrm{Ti}$ yield. Mass fractionating effects were corrected for by measuring the relative beam currents of the stable Ti isotopes $\left({ }^{46-50} \mathrm{Ti}\right)$ for equal velocities at the terminal stripper. Preliminary analysis of the results indicates that the half-life of ${ }^{44} \mathrm{TI}$ is about 10 years longer than the previously accepted value of 47 years.

*I.N.F.N., Legnaro, Italy.

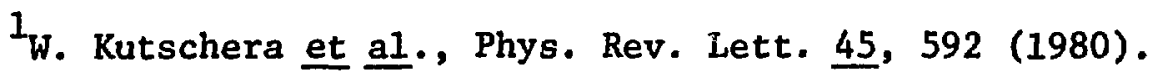




\section{F. SELECTED NUCLEAR SPECTROSCOPY AT THE TANDEM/LINAC}

In addition to the broader areas in heavy-ion physics discussed above, selected light and heavy-ion spectroscopic studies are being performed at the tandem/linac. Foremost among them is a new program in on-line laser spectroscopy which has been initiated recently. The optical hyperfine structure of radioactive atoms will be studied, in order to extract information on spins, moments, and the variation of charge radil for ground states and isomers. The laser system has been installed and tests of the cryogenic helium jet which transport the atoms into the interaction region have been completed. Other studies include mass measurements of neutron-deficient In i.sotopes and a shell-model study of $47 \mathrm{Cr}-47 \mathrm{~V}$ mirror nuclei.

\section{a. laser Spectroscopy of Radioactive Atoms}

C. N. Davids, D. A. Lewis, ${ }^{*}$ R. Evans, ${ }^{*}$ M. A. Finn, ${ }^{\dagger}$ G. Greenlees, ${ }^{\dagger}$ and S. L. Kaufman't

Tests of the cryogenic helium jet have been completed, using radioactivities produced by the Tandem accelerator. The system appears to have an efficiency of between $10-50 \%$ for transporting radioactive nuclei, using pure helium gas as the flow medium. The yield of radioactivity under the skimmer appears to be essentially constant for displacements of the capillary tube off center as much as $1 \mathrm{~mm}$. This result suggests that indeed free atoms are being collected, although confirmation of this fact must await the interaction of laser light with the beam of radioactivities.

A skimmer efficiency of $1.5 \%$ was observed using $\mathrm{Ga}, \mathrm{Ge}$, and As activities produced at the Tandem. This result was measured using a skimmer with a $0.63 \mathrm{~mm}$ dia. hole, and is a factor of 5 larger than the value initially obtained. The increase is due to placing the skimmer opening inside the Mach disk shock front, thus taking advantage of the cooling effect on the atoms of the supersonic expansion.

Diagnostic tests using helium gas seeded with $\mathrm{I}_{2}$ have been performed in the region near the exit of the capillary. Using the $5128 \AA$ light from an $\mathrm{Ar}^{+}$laser, the flow pattern at the exit capillary has been visualized. Figure II-10 shows the jet emerging from the capillary, expanding slightly, then forming the characteristic "barrel shock" pattern just above the skimmer. The glowing region above the laser excitation area is due to fluorescence of the delrin plastic capillary support.

\footnotetext{
* Iowa State University, Ames, Iowa.

University of Minnesota, Minneapolis, Minnesota.
} 


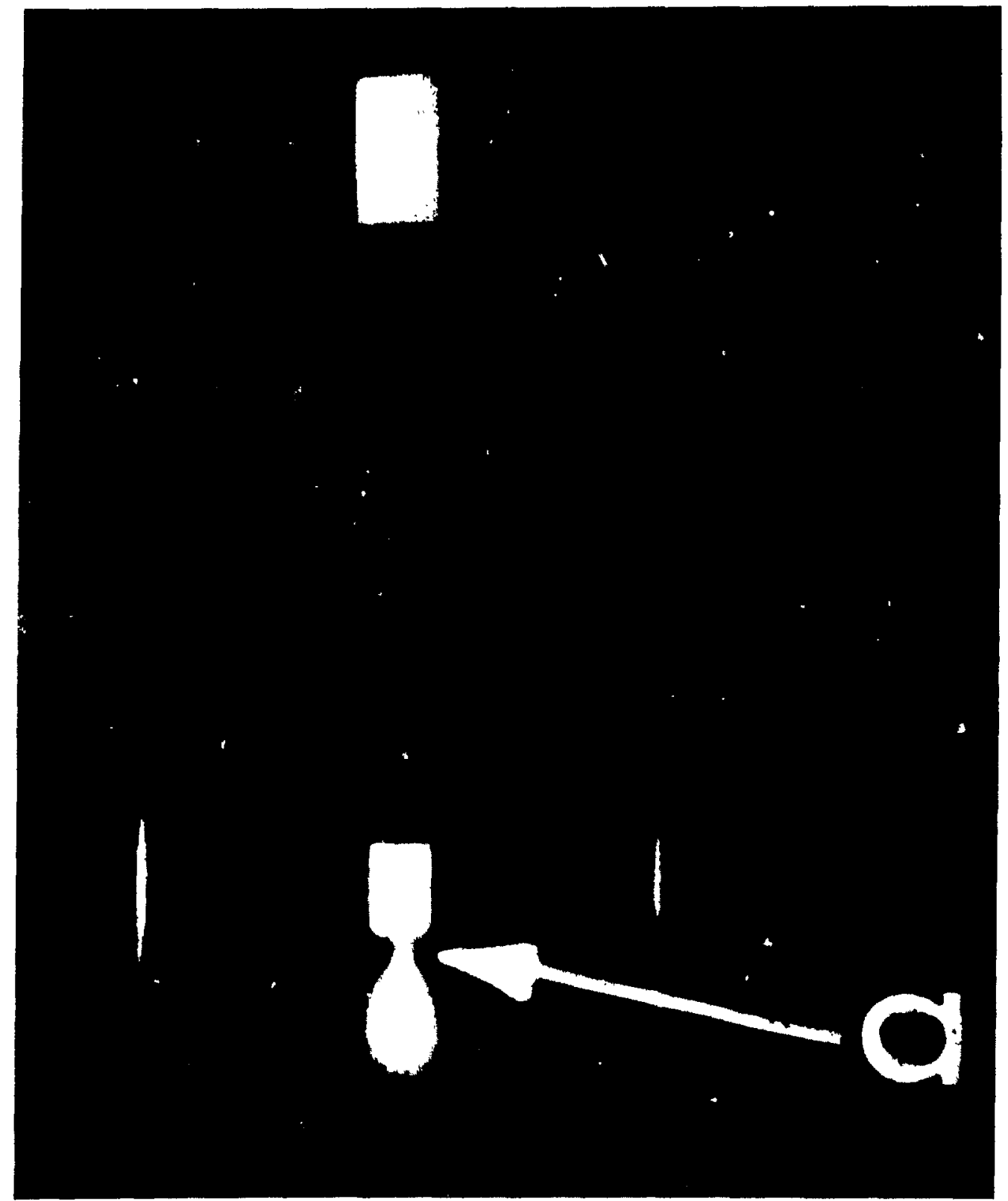

Fig. II-10. Fluorescence of an $\mathrm{I}_{2}$-seeded helium jet by light from an $\mathrm{Ar}^{+}$laser. The arrow points to the exit of the capillary tube. Below the bright expansion core and "barrel" shock can be seen the top portion of the skimmer. 
The laser system has been installed in the new laser laboratory, and $a$ hole has been bored through the wall between it and the target area. Work has begun on the beam line target station. Data acquisition programs for the LSI-11 computer are being modified, and the properties of the laser system are being determined using stable atomic species. Precision measurements made on the $\mathrm{Na}$ Dl line hyperfine splitting show that the laser wavelength scale is correct, and that determinations of isotope shifts in radioactive nuclei will be possible with uncertainties smaller than $0.5 \mathrm{MHz}$.

A new 20-watt $\mathrm{Ar}^{+}$pump laser has been acquired. This will enable measurements to be made in the blue region of the visible spectrum, opening up the possibility of studying the isotope shifts of a number of elements in the rare-earth region of the periodic table.

b. Mass and Low-Lying States of 106,109 In

B. Filippone, ${ }^{*}$ C. N. Davids, R. C. Pardo, and J. Äystö ${ }^{\dagger}$

A study of the masses and low-lying states in the odd-odd nuclei ${ }^{106}$ In and ${ }^{108}$ In via the $\mathrm{Cd}(\mathrm{p}, \mathrm{n} \gamma)$ In reaction has been completed. The masses of these nuclei, which are close to stability, are important to the systematics of the masses of very neutron-deficient nuclei and to calculations of the proton drip 1ine around $\mathrm{Z}=50$. In addition the low-1ying states of odd-odd nuclei provide information on the residual neutron-proton interaction.

For ${ }^{106}$ In a level order has been deduced that places the high-spin beta-decaying state $\left(t_{1 / 2}=6.3 \mathrm{~min}\right)$ as the ground state with a mass excess of $-80601 \pm 12 \mathrm{keV}$. The low-spin beta-decaying state $\left(t_{1 / 2}=5.3 \mathrm{~min}\right)$ has been determined to be an isomer at $28.6 \mathrm{keV}$ above the ground state. Spin assignments for the 14 observed states (including six previously unknown) are made based on previous ${ }^{106}$ Sn beta-decay work and by comparison with statistical model calculations now under way.

For ${ }^{108}$ In the mass excess of the low-spin beta-decaying state $\left(t_{1 / 2}=40 \mathrm{~min}\right)$ has been determined to be $-84018 \pm 12 \mathrm{keV}$. A more detailed study of the low-lying states in ${ }^{108}$ In would provide a better understanding of how the residual interaction causes the high-spin-low-spin isomerism in all of the known even-A indium isotopes.

*Thesis student, University of Chicago, Chicago, Illinois.

tUniversity of Jyvaskyla, Finland. 
c. The $Y$ Decay of States in ${ }^{47} \mathrm{Cr}$

G. Hardie, $^{*}$ S. A. Gronemeyer, ${ }^{\dagger}$ L. Meyer-Schützmeister, ${ }^{\ddagger}$ and A. J. Elwyn

The energy and $\gamma$ decay of states in ${ }^{47} \mathrm{Cr}$, mirror of the much-studied nucleus ${ }^{47} \mathrm{~V}$, were determined. Figure II-11 shows the $\gamma$ decay of some states in the $A=47$ mirror pair. This completes our studies of $T=1 / 2$ mirror nuclei

in the $f_{7 / 2}$ shell. The motivation for the work was to extract Coulomb displacement energies $\left(\Delta E_{c}\right)$ of both natural and unnatural parity states and to study the variation of these $\Delta E_{c}$ with mass number (A). States in ${ }^{47} \mathrm{Cr}$ were studied using the ${ }^{47} \mathrm{Ti}\left({ }^{3} \mathrm{He}, 2 \mathrm{n}\right){ }^{47} \mathrm{Cr}$ reaction with $22-\mathrm{MeV}{ }^{3} \mathrm{He}$ ions and the ${ }^{40} \mathrm{Ca}\left({ }^{12} \mathrm{C}, \alpha \mathrm{n}\right){ }^{47} \mathrm{Cr}$ reaction with $38-\mathrm{MeV}{ }^{12} \mathrm{C}$ ions. Prompt and delayed $\gamma-\gamma$ coincidence experiments were performed. By comparing the energies of the states with the energies of corresponding states in ${ }^{47} \mathrm{~V}$, the $\Delta E_{c}$ were extracted. For a given $J^{\pi}$, the $\Delta E_{c}$ were found to increase with increasing $A$. To try to understand the above results a shell-model calculation was performed assuming a pure $\left(\mathrm{f}_{7 / 2}\right)^{7}$ configuration. The level structure predicted by this calculation agrees quite well with the measured one. An exception is the $3 / 2^{-}$state, which surely contains a large $\left(1 f_{7 / 2}\right)^{6}\left(2 p_{3 / 2}\right)^{1}$ component. The model is also quite successful in reproducing details of the trend of $\Delta E_{c}$ with $A$. The measured energies of the first $3 / 2^{+}$states in $T=1 / 2$ mirror pairs of $f_{7 / 2}$ shell nuclei were used to determine the average Coulomb interaction energy between $a_{3 / 2}$ hole and an $f_{7 / 2}$ particle. This energy was found to increase in magnitude with increasing $A$. We have no explanation for this behavior.

\footnotetext{
*Western Michigan University, Kalamazoo, Michigan.

†ermi National Accelerator Laboratory, Batavia, Illinois.

$\ddagger_{\text {Deceased. }}$
} 


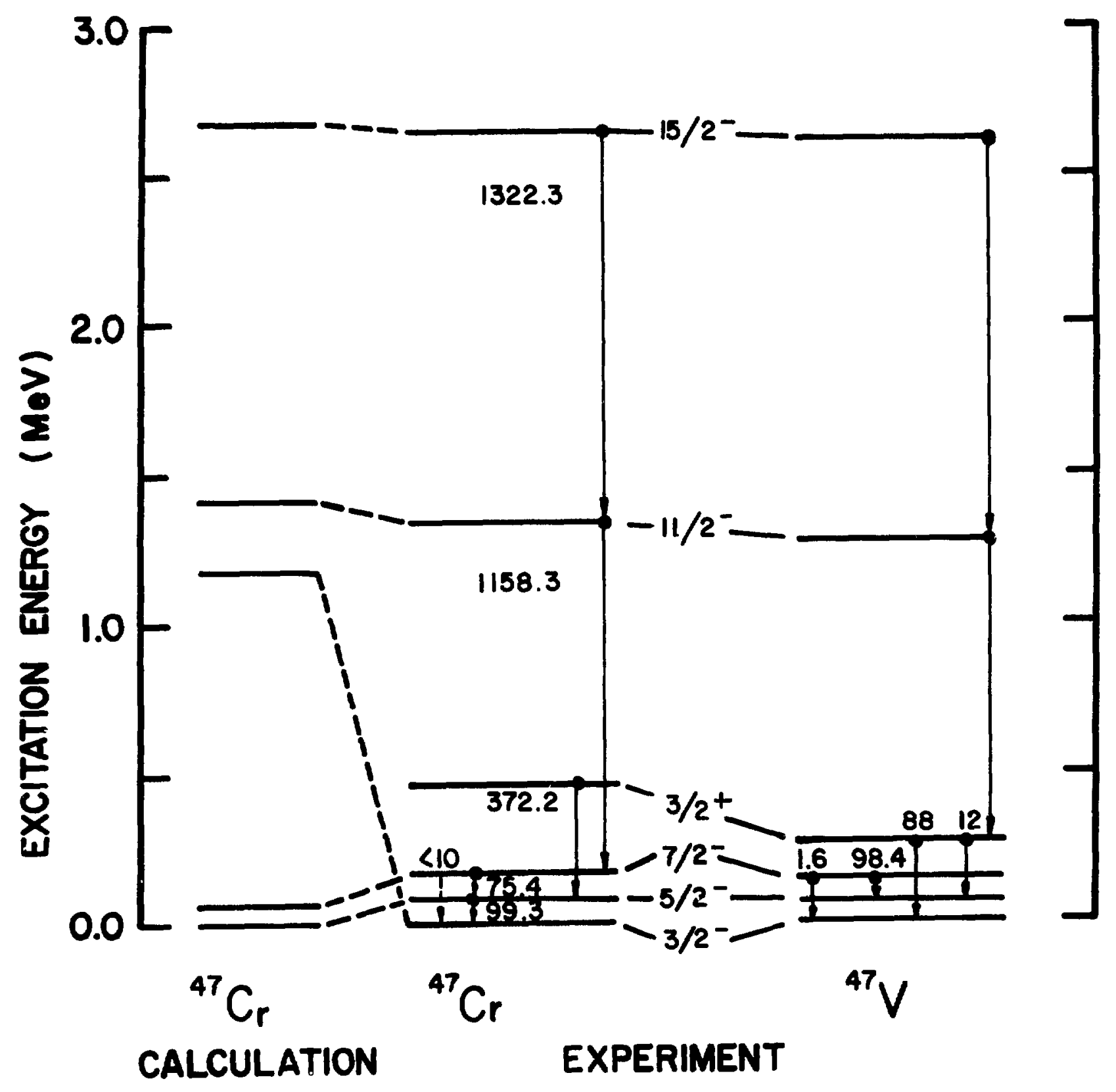

Fig. II-11. Gamma decay of some states in the A=47 mirror pair. The data for $47 \mathrm{Cr}$ are from the present work, while those for $47 \mathrm{~V}$ are from the literature. The calculation was performed by A. Amusa and R. D. Lawson at ANL. 


\section{G. EQUIPMENT DEVELOPMENT}

The experimental capabilities in the target area of the linac booster have been significantly expanded by the completion of the splitpole magnetic spectrograph and installation of the laser-helium jet system for laser spectroscopy of radioactive atoms. Operating the post-linac buncher in its debunching mode for the first time, excellent energy resolution has been observed in first tests of the split-pole spectrograph with beams from the linac. A new beam line has been installed with a thin-walled aluminum chamber for neutron time-of-flight measurements, which will be used in experiments starting spring 1982. Work has also progressed on the design and construction of the Purdue superconducting electron spectrometer, which is scheduled for initial on-line tests in the middle of 1983 . The 65-in. scattering chamber has been upgraded to provide long flight paths for charged-particle time-of-flight measurements and a new electrostatic deflector for use in fusion measurements in heavy systems has been built and is operating reliably at $50 \mathrm{kV}$. A plunger for recoil distance lifetime measurements has been constructed and successfully tested on-line.

a. The Split-Pole Magnetic Spectrograph in the Linac Experimental Area

W. Kutschera, K. E. Rehm, T. Humanic, D. G. Kovar, and R. Pardo

The installation of the Enge split-pole magnetic spectrograph has been completed and tests with beams from the linac of ${ }^{12} \mathrm{C}(108 \mathrm{MeV}),{ }^{32} \mathrm{~s}$ $(225 \mathrm{MeV})$, and ${ }^{60} \mathrm{Ni}(300 \mathrm{MeV})$ performed. The spectrograph is equipped with an all-cryogenic pumping system producing a vacuum of $10^{-7}$ Torr in the target chamber and $10^{-6}$ in the detector box. The ionization chamber focal plane detector, successfully used for many years with the tandem spectrograph, is used as the basic heavy-ion detector. Using a $108-\mathrm{MeV}$ debunched ${ }^{12} \mathrm{C}$ beam from the linac, an energy resolution of $\Delta E / E=10^{-3}$ has been measured.

The spectrograph is now fully operational and first experiments have been started. The special features of this new facility-large dynamic range, high resolution and reasonable solid angle-will considerably broaden the experimental program. A deeper gas detector is presently being tested which will allow exploitation of the highest energy beams from the linac. A modification of the target chamber has been completed to bring a Ge(Li) detector very close to the target in order to perform heavy-ion-gamma coincidence experiments. It is planned to increase the versatility of the instrument further by implementing a mini-orange type spectrometer for heavy ion-conversion electron coincidence experiments. 
b. The $y$-Ray Facility

S. R. Faber, ${ }^{*}$ T. L. Khoo, and J. N. Worthington

The first phase of development on a $\gamma$-ray facility is now essentially complete. It includes a sum spectrometer (two 13-in. $\times$ 6-in. NaI crystals), several target chambers, rotatable detector mounts with angle readout, a collection of Ge detectors and several large (10-in. × 12-in.) NaI crystals. The $\gamma$ target station has been moved to a new beam line resulting in more space for detection systems.

A stabilization system for the NaI detectors, capable of maintaining the photomultiplier gain to within $0.25 \%$, has been constructed. Stabilization is referenced with respect to light from an LED, which is fanned out to the NaI photomultipliers by optic fibers. The output of the LED itself is monitored with a PIN diode and stabilized with a feedback circuit.

A plunger which fits inside the sum spectrometer has just been constructed and is described in the next section. Design work on a beam line to accommodate the Purdue electron spectrometer has also been initiated. Design studies of a bismuth germanate (BGO) anti-Compton spectrometer are under way.

${ }^{*}$ Purdue University, West Lafayette, Indiana.

\section{c. A Plunger for Recoil Distance Lifetime Measurements}

T. L. Khoo and W. A. Huston

A plunger for recoil distance lifetime measurements has been constructed. The plunger is small and compact enough to be operated within our NaI sum spectrometer. The design is different from that of any previous plunger. The catcher foil in our instrument is held on 3 independent Invar push rods, which are driven by microprocessor-controled d.c. motors (purchased from Newport Research). The catcher foil may be remotely positioned to a precision of less than $1 \mu \mathrm{m}$, and its parallelism with respect to the target may also be altered while in vacuum. Initial tests, including an in-beam measurement, have been completed. The plunger will first be used for measuring 1 ifetimes in ${ }^{154}$ Dy, where the complexity of the $\gamma$ spectrum may be reduced by use of the sum spectrometer. Feeding times of high spin yrast states in ${ }^{152}$ Dy will also be measured as a function at the $\gamma$-sum energy. 


\section{d. Sunarconducting Solenoid Lens Electron Spectrometer}

P. J. Daly, ${ }^{*}$ Z. Grabowski, ${ }^{\text {t }}$ R. V. F. Janssens, and T. L. Khoo

Work has progressed for the construction of the Purdue superconducting electron spectrometer to be installed at the linac. The design of the superconducting coils has been completed at Purdue and the basic coriponents for the spectrometer have now been ordered. Delivery is expected during the summer of 1982 and final assembly will then proceed. Meanwhile, the basic layout of a new beam line has been completed at Argonne. It is hoped to perform initial on-line tests in the middle of 1983. The spectrometer will first be used to measure conversion coefficients for transitions in $150 \mathrm{Er}, 151,152,153^{\mathrm{Dy}}$. In these nuclei currently under investigation at the Laboratory, long-living high spin isomers and complex level schemes make the use of conversion electrons essential to establish the multipole character of transitions crucial for the interpretation of nuclear structure at high spin. A variety of other experiments are planned, some of which will take advantage of the possibility of measuring $e^{-}-e^{-}$coincidences.

*Purdue University, West Lafayette, Indiana.

\section{e. Design Study of a BGO Anti-Compton Spectrometer}

M. Mohsen, ${ }^{*}$ T. L. Khoo, and R. V. F. Janssens

Bismuth germanate (BGO) has a $\gamma$-ray absorption coefficient $2-3$ tines larger than that of $\mathrm{NaI}$, and thus is an attractive material for a compact anti-Compton shield. The use of Compton-suppressed Ge spectrometers will provide cleaner spectra for our pursuit of the highest spin yrast states. We have undertaken initial studies on the optimal BGO crystal size for Compton uppression. Emphasis is placed on a design which would allow several (up to 6) Compton-suppressed systems to be placed around the targat. We find that 5-in. $\times$ 5-in. BGO crystals will provide good Compton suppression. These will be within present manufacturing capability by mid-1982.

*University of King Abd E1 Aziz, Geddah, Saudi Arabia. 


\section{f. Nuclear Target Making and Development}

G. E. Thomas

The Physics Division target facility produces very thin targets for experiments at the tandem-linac and Dynamitron accelerators, for experiments of other members of the Division, any other division at the Laboratory needing this service and occasionally for other laboratories and universities.

During this year the different elements, isotopes or compounds evaporated, rolled, anodized or oxidized included $\mathrm{Al}$, $\mathrm{Au}, \mathrm{Al}_{2} \mathrm{O}_{3}, 10,11, \mathrm{nat} \cdot \mathrm{B}$, $\mathrm{Bi}, \mathrm{BaCl}_{2}, \mathrm{Be}, \mathrm{C}, 63$, normal $_{\mathrm{Cu}}, \mathrm{CaF}_{2}, 40$, nat. $\mathrm{Ca}, 58$, nat.Fe, 6, 7, nat. $\mathrm{LiF}, \mathrm{MgO}$, 24 , nat. $\mathrm{Mg}, \mathrm{MgF}_{2}, 95,100 \mathrm{Mo}, \mathrm{Ni}+\mathrm{Au}, 58$, nat. $\mathrm{Ni},{ }^{15} \mathrm{NH}_{4} \mathrm{NO}_{3}, \mathrm{NaCl},{ }^{9} \mathrm{Nb}$, 208 , nat. $\mathrm{Pb}, 112,116,118,120,122,124 \mathrm{Sn}, \mathrm{Si}, 126,130 \mathrm{Te}, \mathrm{Ta}, \mathrm{WO}_{3}, 90,92,94 \mathrm{Zr}$. Standards were produced from $10,11_{B}$ and $\mathrm{Ni}$ consisting of 2.5,5, 25,50, and 100 monolayers deposited on $50 \mu \mathrm{g} \mathrm{cm}^{-2}$ of $\mathrm{Al}$.

Targets which are made for experiments at the heavy-ion facility are now often much more complex and time consuming to produce. They may be multilayer targets or isotopes $\sim 1 \mathrm{mg} \mathrm{cm}^{-2}$ evaporated onto very tautly stretched $1 \mathrm{mg} \mathrm{cm} \mathrm{cm}^{-2} \mathrm{Au}$. New techniques have been developed for making various other types of targets. Also we are actively involved in producing longer-lived carbon stripper foils for the accelerator.

Our third evaporation system has been completed and is now in routine use and performs quite well, giving us a much more flexible facility. Plans for the future include the conversion of one evaporation system for use in making actinide targets. The fabrication of the multitarget evaporation system is also in the future. 


\title{
III. CHARGED-PARTICLE RESEARCH
}

\author{
INTRODUCTION
}

The activities in nuclear research include measurements of low-energy cross sections crucial to the understanding of solar neutrinos associated with energy production in the sun, the study of the properties of the weak interaction, and a search for stable fractionally-charged particles, all associated with the Argonne Dynamitron. The weak interactions program has recently expanded its scope and is a vital part of a large collaboration to search for neutrino oscillations* at LAMPF. An experiment is being developed to measure the electric dipole moment of the neutron. Well over half of the nuclear research work is dedicated to charged-particle research at the tandem-superconducting linac accelerator. That research is described under Heavy-Ion Research, with which it is closely coupled scientifically.

*

A report of this activity is included in the Medium-Energy Physics section under Sec. I.D. 


\section{CHARGED PARTICLE RESEARCH AT THE DYNAMITRON AND WEAK INTERACTIONS AT LOW ENERGY}

The nuclear physics research at the Dynamitron derives its scientific motivation from two principal sources. One of the programs treats the nucleus as a microscopic laboratory for the investigation of fundamental processes. At present this research is focused on aspects of the weak interactions that can be observed in nuclear decays. Unfortunately, a large ef fort in this area, the measurement of the parity mixing between the 5.11- $\mathrm{MeV} J \pi=2^{-}$, $T=0$ and $5.16-\mathrm{MeV}, \mathrm{J}^{\pi}=2^{+}, T=1$ levels in $10_{\mathrm{B}}$ had to be abandoned because of severe difficulty encountered in producing an adequate polarized ${ }^{6} \mathrm{Li}$ target.

The beta decay branch of the excited ${ }^{16} \mathrm{~N}, 120-\mathrm{keV} \mathrm{J} \mathrm{J}^{\pi}=0^{-}$state to the ${ }^{16} \mathrm{O}, \mathrm{J}^{\pi}=0^{+}$ground state was measured to $12 \%$ accuracy. This decay provides direct and compelling evidence for the important role of pion exchange currents in time-like axialmvector decay. The result of this $16_{\mathrm{N}}$ decay experiment when coupled with the inverse $\mu^{-}$capture provides a determination of the induced pseudoscalar coupling constant $\left(g_{p}\right)$; one finds $g_{p} / g_{A}=10.5 \pm 2.5$. Searches for fundamental particles such as quarks and axions are also part of this activity.

The other area of nuclear research at the Dynamitron uses the expertise built up at ANL over the past 5 years on measurements of highly exothermic reactions involving light nuclei. This expertise is being used to investigate important elements of the nuclear synthesis chain responsible for solar neutrinos. The purpose of these experiments is to remove significant sources of uncertainty in nuclear reaction data used in the calculition of the solar neutrino flux. Measurements performed over the last year show that the earlier values used for a calibration reaction $7_{\mathrm{Li}}(\mathrm{d}, \mathrm{p})^{8} \mathrm{Li}$ were in error by $15 \%$. This reduces the predicted solar neutrino rate from $7 \pm 3$ SNU to $6 \pm 2.5 \mathrm{SNU}$ as compared to the rate observed in the BNL Cl detector of $2.2 \pm 0.4 \mathrm{SNU}$. A close coupling between the astrophysics program at the University of Chicago and this research area is proving to be mutually beneficial.

\section{WEAK TNTERACTIONS}

a. Parity Violation in the $5.1-\mathrm{MeV} J=2$ Doublet of ${ }^{10} \mathrm{~B}$

C. A. Gagliardi,* G. T. Garvey, and S. J. Freedman ${ }^{\dagger}$

Since the discovery of the neutral weak current, there has been a great deal of effort to define its character. To date, the leptonic and semileptonic results are well described by the $S U(2) \times U(1)$ gauge theory

*Thesis student, Princeton University, Princeton, New Jersey.

${ }^{\dagger}$ In part from Stanford University, Stanford, California. 
of Weinberg-Salam with a mixing angle $\sin ^{2} \theta_{w} \sim 0.23$. Studies of the purely hadronic neutral current have been more ambiguous.

The neutral current interaction between hadrons must be studied by determining the size of parity violating processes. Study of the parity mixing between states with $T=0$ and $T=1$ suppresses the effects of the charge-changing weak current by $\sin ^{2} \theta_{c}=0.05$ and thus is especially favorable for cleanest examination of NWC effects. Despite extensive efforts, no such mixing has yet been observed in an interpretable nuclear system. We have attempted to determine the mixing between the $2^{-}, T=0,5.11-\mathrm{MeV}$ and $2^{+}, \mathrm{T}=1$, $5.16-\mathrm{MeV}$ levels in ${ }^{10} \mathrm{~B}$. The experiment was to employ a 1.2-MeV $\alpha$-particle beam from the Dynamitron accelerator to bombard a polarized ${ }^{6} \mathrm{Li}$ target. Ihe $5.16-\mathrm{MeV}$ state is to be resonantly formed and observed via the ${ }^{6} \mathrm{~L} \vec{I}(\alpha, \gamma){ }^{10_{B}}$ reaction. The yield is given by $\sigma=\sigma_{0}\left(l+\frac{1}{2} \mathrm{P}_{z z}+\mathrm{HBP}_{z}\right)$, where the $z$ axis is taken along the incident $\alpha$-particle beam. In the above expression, $P_{z}$ and $P_{z z}$ are the vector polarization and the tensor alignment of the ${ }^{6} \mathrm{Li}$ beam, respectively, $B$ is the parity mixing amplitude, and $H$ the enhancement factor due to the different widths of the states.

The principal technical difficulty in the experiment involves producing a polarized ${ }^{6} \mathrm{Li}$ target $10^{14}-10^{15}$ atoms $/ \mathrm{cm}^{2}$ thick. The technique employed involved producing a polarized ${ }^{6} \mathrm{Li}$ atomic beam and collecting it on a hot $\left(1100^{\circ}-1500^{\circ} \mathrm{C}\right)$ oxidized tungsten surface. The ${ }^{6} \mathrm{Li}$ dwell time is sufficiently short to not allow depolarization but long enough $\left(10^{-2}\right.$ sec) to permit the accumulation of sifficient ${ }^{6} \mathrm{Li}$. Unfortunately, severe difficulty was encountered in the developmeni af this target. Trace amounts of ${ }^{6} \mathrm{Li}$ $\left(10^{-2} \mu \mathrm{g} / \mathrm{cm}^{2} \mathrm{hr}\right)$ apparently became tcapped in grain boundaries at the near surface. The yield from this source of unpolarized ${ }^{6} \mathrm{Li}$ becomes comparable with the yield from the polarized ${ }^{b_{L i}}$ within 30-60 niin. Furthermore, the size of the predicted asymmetry $\left(\left[\sigma\left(\mathrm{P}_{z}\right)-\sigma\left(-\mathrm{P}_{z}\right)\right] / \sigma_{0}\right)=2 \mathrm{HBP}$ dropped from approximately $10^{-3}$ to approximately $10^{-4}$ as sma.11 but important nuclear structure effects had been neglected in the earlier calculations in the literature. Insofar as its 6 it target developmeni has proved to be far more difficult than anticipated and the estimated size of the effect is now ten times smaller, it was felt that the most productive course of action is to terminate further activity on this problem at this time. Should a practical method for producing a suitable target present itself, we would certainly wish to pursue this measurement. 
b. The Beta Decay Rate of $\left.{ }^{16} \mathrm{~N}^{-}, 120 \mathrm{keV}\right):$ Meson Exchange Currents and the Induced Pseudoscalar Coupling Constant

C. A. Gagliardi, * G. T. Garvey, S. J. Freedman, ${ }^{\dagger}$ and J. R. Wrobel

We have remeasured the beta decay rate of ${ }^{16} \mathrm{~N}\left(0^{-}, 120 \mathrm{keV}\right)$ to $\left.{ }^{16} \mathrm{o}_{\left(0^{+}\right.}, \mathrm{g.s.}\right)$. The dominant contribution to this $0^{-} \rightarrow 0^{+}$transition comes from the time component of the weak axial vector current. Recently, it was shown $^{1}$ that the time component of the axial current should receive a large ( $250 \%$ ) enhancement from pion-exchange contributions. For the case of ${ }^{16} \mathrm{~N}\left(0^{-}\right)$, detailed calculations ${ }^{2}$ indicate that the predicted $0^{-} \rightarrow 0^{+}$beta decay rate $\Lambda_{\beta}$ increases a factor of 3-5 when pion-exchange currents are included in addition to the normal one-body impulse approximation terms. Meanwhile, it has been demonstrated ${ }^{2}$ that the beta decay rate may be combined with the $\mu$-capture rate $\Lambda_{\mu}$ for the inverse reaction to determine the induced pseudoscalar coupling constant $g_{p}$ in finite nuclei in a manner which is insensitive to uncertainties in the nuclear model calculations. The only previous measurement ${ }^{3}$ of $\Lambda_{\beta}$ found $\Lambda_{\beta}=(0.46 \pm 0.10) \mathrm{sec}^{-1}$. This value is suggestive of the need for pion-exchange currents, but the large experimental uncertainty and the lack of confirmation reduce one's confidence in this conclusion.

For our experiment, we constructed a rotating target system that allows eight separate concentric circular tracks of ${ }^{15} \mathrm{NH}_{4}^{1.5} \mathrm{NO}_{3}$ to be placed in the Dynamitron beam sequentially. ${ }^{16} \mathrm{~N}$ is produced in the reaction ${ }^{15} \mathrm{~N}(\mathrm{~d}, \mathrm{p}){ }^{16} \mathrm{~N}$ with a $30 \mu \mathrm{A} 3.4-\mathrm{MeV} \mathrm{d}{ }_{2}{ }^{+}$beam from the Dynamitron. The beam irradiates the target for $10 \mu \mathrm{s}$, after which $\beta$ and $\gamma$ decays of ${ }^{16} \mathrm{~N}\left(0^{-}, 120\right.$ $\mathrm{keV}$ ) are observed during a $63 \mu \mathrm{s}$ long counting period. $\beta$ rays are detected with a telescope consisting of four thin plastic scintillators operated in coincidence. ${ }^{16} \mathrm{~N}$ beta decays are recognized by their characteristic lifetime of $7.58 \mu \mathrm{s}$. $120-\mathrm{keV} \gamma$ rays are detected with a $\mathrm{Ge}(\mathrm{Li})$ detector to normalize the source activity. The high current, low-energy deuteron beam together with the multitrack target lead to a data rate a factor of 11 greater than in

*Thesis student, Princeton University, Princeton, New Jersey.

'In part from Stanford University, Stanford, California.

$1_{K}$. Kubodera, J. Delorme, and M. Rho, Phys. Rev, Lett. 4n, 755 (1978).

2I. S. Towner and F. C. Khanna, Nuc1. Phys. A372, 331 (1981).

3 L. Palffy, J. P. Deutsch, L. Grenacs, J. Lehmann, and M. Steels, Phys. Rev. Lett. 34, $212(1975)$. 


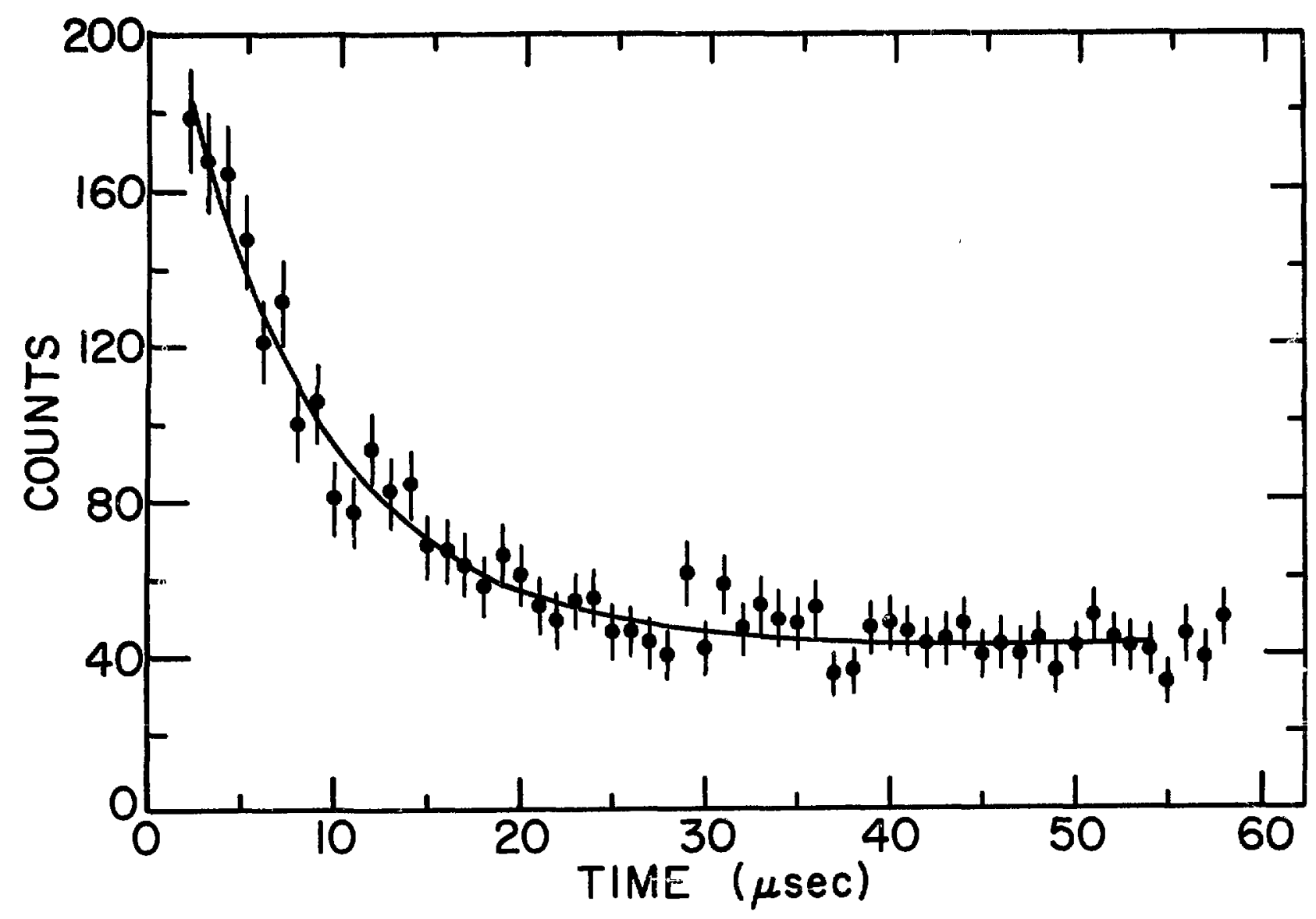

Fig. III-1. A typical beta detector time spectrum. The smooth curve is a three-parameter fit to the data of the form $\mathrm{Ae}-t / \tau_{m}+B$. The extracted lifetime is $\tau_{m}=(7.9 \pm 0.6) \mu \mathrm{s}$, in good agreement with the accepted lifetime of the $0^{-}$state, and the $x^{2}=38$ for 50 degrees of freedom.

the previous experiment, while the signal-to-noise ratio is increased a factor of 3 . Figure III-1 shows a typical $\beta$ detector time spectrum. The efficiency of the $\mathrm{Ge}(\mathrm{LI})$ detector at $120 \mathrm{keV}$ is moasured with calibrated radicactive sources, and the beta detector efficiency for ${ }^{16} \mathrm{~N}\left(0^{-}\right) \rightarrow{ }^{16} 0\left(0^{+}\right.$, g.s.) $B$ rays is measured by observing decays of ${ }^{16} \mathrm{~N}\left(2^{-}\right.$, g.s.) to ${ }^{16} \mathrm{o}\left(0^{+}\right.$, g.s. $)$. We find $\Lambda_{\beta}=(0.45 \pm 0.05) \mathrm{sec}^{-1}$, consistent with the previous measurement and with the need for pion-exchange currents. The present experimental uncertainties in the beta decay branching ratios of ${ }^{16} \mathrm{~N}\left(2^{-}\right.$, g.s. $)$dominate our uncertainty, contributing $10 \%$. The value of $\Lambda_{\mu} / \Lambda_{\beta}$ implies $g_{p} / g_{A}=11 \pm 1.5$. Figure III-2 shows the experimental values of $\Lambda_{\mu}$ and $\Lambda_{\beta}$, along with the theoretical predictions from Ref. 2 . 


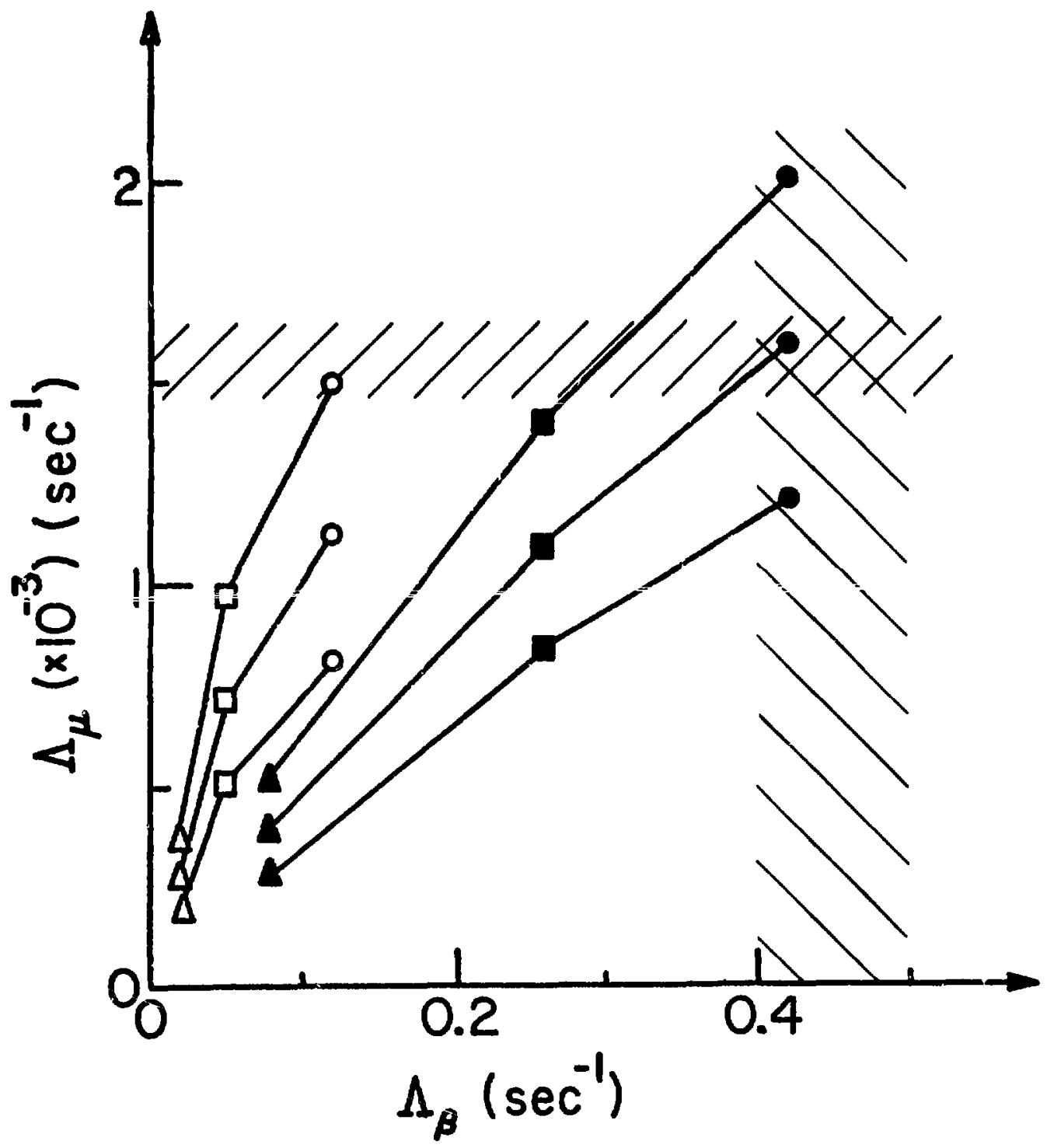

Fig. III-2. A comparison of the experimentally allowed values of $\Lambda_{\mu}$ and $\Lambda_{B}$ with the predictions of Ref. 2. The open points are nucleons-only impulse approximation calculations, while the closed points include the effects of pion-exchange currents. Three different residual interactions are used to define the nuclear wavefunctions, and the calculations are shown for three values of $g_{\mathrm{p}} / \mathrm{g}_{\mathrm{A}}(7,10,13)$. 


\section{c. Beta Spectrum Measurements}

A. R. Heath ${ }^{*}$ and G. T. Garvey

The measurement of beta spectra sufficiently accurate and reliable to see "weak magnetism" has proven difficult. Although a great deal of attention was focused on this subject in the mid-1960's, it is not without continuing controversy. Only recently has the spectrum of the supposedly trivial $0^{+} \rightarrow 0^{+}$decay been measured to a sufficient accuracy $(0.4 \% / \mathrm{MeV})$ required to see "induced current" effects. Many recent efforts have failed to measure a "weak magnetism" term because of experimental uncertainties. In addition, accurate measurements of $0^{+} \rightarrow 0^{+}$beta shape factors will allow an examination of the procedures used for radiative corrections and a better limit to be set on the Fierz interfererce term which arises from possible scalar coupling.

A system using modern techniques employing a flat field magnet, position sensitive proportional counters and active slits has to be designed.

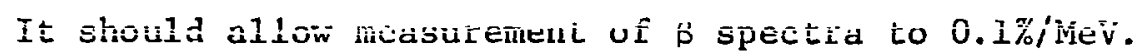

We have procured, tested, and field mapped the flat field magnet. The entire vacuum system for the experiment has been constructed and put on the Dynamitron beam line. A three wire drift chamber has been built and tested with the magnet under experimental conditions and yields a resolution of $2 \mathrm{~mm} /$ wire and shows background not to be a problem. The only element to be fabricated is the active slit, and design of that element is under way. As soon as the entire system is functioning, it will be used to measure the ${ }^{16} \mathrm{~N}$ beta system as well as the purpose it was designed for.

\section{d. Beta Decay Branching Ratios of ${ }^{16} \mathrm{~N}\left(2^{-}\right)$}

A. R. Heath ${ }^{*}$ and G. T. Garvey

An effort is underway at present to determine the beta decay rate

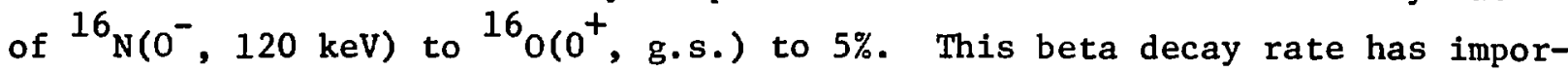
tant implications for the presence of pion-exchange contributions to the weak axial vector current and for the value of the induced pseudoscalar coupling constant. The technique used to calibrate the beta detector efficiency in that experiment requires knowledge of the beta decay branching ratios of the ${ }^{16} \mathrm{~N}^{-}$ground state. The present experimental uncertainty in the quantity $\left.\mathrm{BR}^{16} \mathrm{~N}\left(2^{-}\right) \rightarrow 6.13 \mathrm{MeV} \gamma \mathrm{ray}\right] / \mathrm{BR}\left[{ }^{16} \mathrm{~N}\left(2^{-}\right) \rightarrow{ }^{16} \mathrm{O}\left(0^{+}, \mathrm{g} . \mathrm{s}.\right)\right]$,

\footnotetext{
* Thesis student, University of Chicago, Chicago, Il]inois.
} 
which appears in the calibration, is $11 \%$ and dominates the overall uncertainty in the ${ }^{16} \mathrm{~N}\left(0^{-}\right)$beta decay rate. We intend to use the magnetic $\beta$-ray spectrometer that is currently under development to remeasure these branching ratios. Our goal is to reduce the experimental uncertainty a factor of 4 . At that point, the overall uncertainty in the ${ }^{16} \mathrm{~N}\left(0^{-}\right)$beta decay branch will be dominated by the statistical accuracy in the observed number of decay eiectrons.

e. Bata Decay of ${ }^{16} \mathrm{C}$

C. A. Gagliardi, G. T. Garvey, R. G. H. Robertson, * and W. Jarmie* We are remeasuring the beta decay of ${ }^{16} \mathrm{C}$. This activity decays primarily to neutron-unstable states of ${ }^{16} \mathrm{~N}$. The decay of ${ }^{16} \mathrm{C}$ to ${ }^{16} \mathrm{~N}\left(0^{-}\right.$, $120 \mathrm{keV}$ ) has not been observed previously. The rate of this $0^{+} \rightarrow 0^{-}$firstforbidden beta decay is expected to receive a large enhancement from pionexchange contritutions to the weak axiai-vector time component. The oniy previous study ${ }^{1}$ of ${ }^{16} \mathrm{C}$ beta decay set an upper 1 imit of $<0.5 \%$ for this branch. The ${ }^{16} \mathrm{C}$ was produced in the reactions ${ }^{14} \mathrm{C}\left({ }^{18} \mathrm{O},{ }^{16} \mathrm{O}\right){ }^{16} \mathrm{C}$ and ${ }^{11}{ }_{B}\left({ }^{7} \mathrm{Li}, 2 \mathrm{p}\right){ }^{16} \mathrm{C}$. Background activities produced simultaneously limited the experimental sensitivity. In the present study, ${ }^{16} \mathrm{C}$ is produced in the reaction ${ }^{14} \mathrm{C}(\mathrm{t}, \mathrm{p}){ }^{16} \mathrm{C}$, using a triton beam from the Los Alamos Tandem Van de Graaff. The target is observed simultaneously by beta and neutron detectors, in addition to an intrinsic-germanium detector, o count the $120-\mathrm{keV} \gamma$ rays following the beta decay of ${ }^{16} \mathrm{C} \rightarrow{ }^{16} \mathrm{~N}\left(0^{-}\right)$. We expect to be sensitive to a beta decay branching ratio to ${ }^{16} \mathrm{~N}\left(0^{-}\right)$as small as $0.01 \%$.

* Los Alamos National Laboratory, Los Alamos, New Mexico.

${ }^{1}$ D. E. Alburger and D. H. Wilkinson, Phys. Rev. C 13, 835 (1976).

\section{f. A Search for Axions from Nuclear Decays}

S. J. Freedman and K. T. Knöpfle*

Recent positive experimental results have stimulated new interest in the possibility that axions (a light pseudoscalar "semiweak!y" interacting "higgslike" fundamental particle) may actually exist. The axion is expected to be rroduced in competition with certain nuclear gamma decays and we have undertaken to look for them exploiting an elaborate gamma-ray detector

\footnotetext{
* Max Planck Instituce, Heidelberg, Germany.
} 
(the crystal bal1) developed at the Max Planck Institute. The experiment proposed, to be carried out in Heidelberg during 1982, will search for two photon decays of the axions emitted in nuclear transitions. The experiment is simple: an intense radioactive source at the center of a high density shielding sphere is placed at the center of the crystal ball. Two photon decays are detected in the crystal ball which consists of about 150 separate NaI(T1) crystals. Backgrounds from cosmic rays are controled by cuts that enhance the characteristic decay of the axion. The first radioactive sources to try will be ${ }^{51} \mathrm{Cr}$ and ${ }^{7} \mathrm{Be}$.

\section{REACTIONS USING LIGHT NUCLEI}

The main focus of this program has been on nuclear reactions of interest to astrophysics. Two independent measurements of the ${ }^{\mathrm{Li}(d, p)}{ }^{8} \mathrm{Li}$ cross section at the $0.78-\mathrm{MeV}$ resonance have already yielded important results bearing on the filux of neutrinos from the sun. The predicted rate of solar neutrino captures at the earth is reduced by $215 \%$ when the new cross-section value is used to normalize previous measurements of the $7_{\mathrm{Be}}(\mathrm{p}, \gamma)^{8} \mathrm{Be}$ cross section. A study of the important $7 \mathrm{Be}(\mathrm{p}, \gamma)^{8} \mathrm{~B}$ reaction has begun, using a new technique for producing uniform $7 \mathrm{Be}$ targets. Complementary calculations of the effect on the neutrino capture rate of variations of the input parameters have been carried out for the current ${ }^{37} \mathrm{Cl}$ experiment and the proposed $71_{\mathrm{Ga}}$ experiment. A study of radiative proton capture on $27 \mathrm{Al}$ and $19 \mathrm{~F}$ has begun, with the objective of observing the nonresonant $\gamma$-ray yield at bombarding energies $\leqslant 1 \mathrm{MeV}$. At these energies, nonresonant processes are expected to arise predominantly from a direct-capture mechanism.

\section{a. Uncertainties in the Calculation of the Solar Neutrino Flux}

B. Filippone ${ }^{*}$ and D. N. Schramm ${ }^{\dagger}$

A detailed examination of the uncertainties in the calculation of the solar neutrino flux has been completed. ${ }^{1}$ The motivation for such a study is to investigate what effects recent changes in the input physics of a standard solar model have on the calculated neutrino capture rates in both the on-1ine ${ }^{37} \mathrm{Cl}$ experiment and the proposed ${ }^{71_{\mathrm{Ga}}}$ experiment.

The "solar neutrino problem"-the discrepancy between the calculated and observed neutrino flux from the nuclear reactions in the interior of the

\footnotetext{
* Thesis student, University of Chicago, Chicago, Illinois.

${ }^{\dagger}$ University of Chicago, Chicago, Illinois.

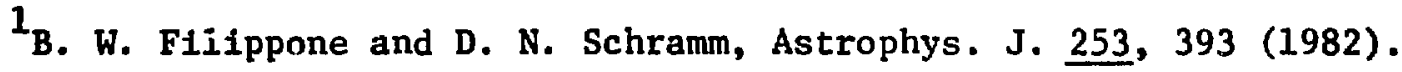



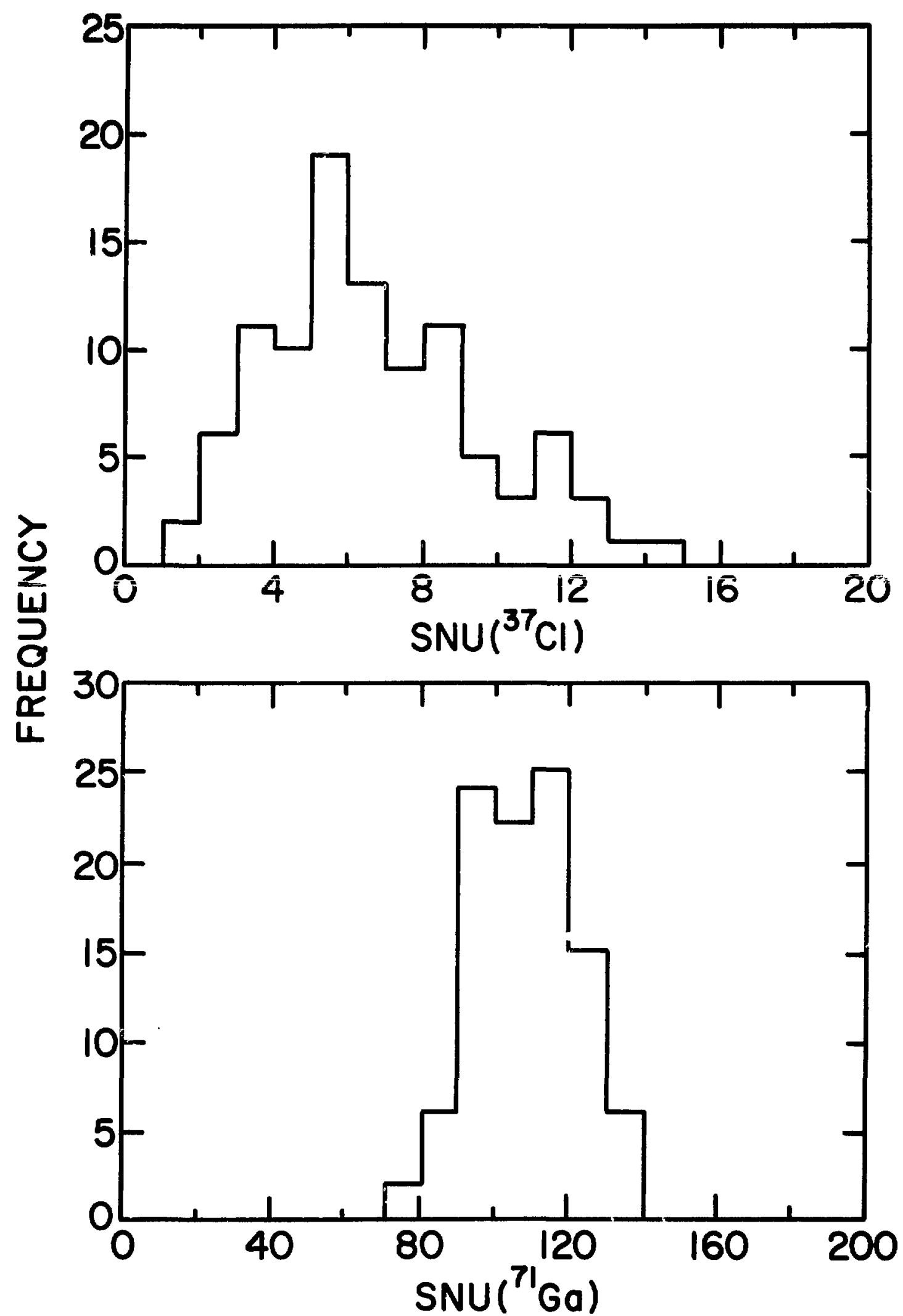

Fig. III-3. Shown are the results of the Monte Carlo calculations for the ${ }^{37} \mathrm{Cl}$ and ${ }^{71} \mathrm{Ga}$ detector. 
sun-has plagued astrophysics for some 15 years. Recent experimental and theoretical indications of neutrino oscillations also prompt a reexamination of solar neutrino calculations in order to see what might be learned of neutrino physics from this discrepancy.

This uncertainty in the calculation has been examined in a novel way by a Monte Carlo method where the input parameters of the model have been simultaneously varied about their mean within assigned uncertainties. This method is important because of the highly nonlinear dependence of the high-energy part of the neutrino spectrum on the various parameters. The results for the ${ }^{37} \mathrm{Cl}$ detector are $7.0 \pm 3.0$ SNU (SNU $\equiv$ Solar Neutrino Units $=10^{-36} \nu$ captures/target atom-sec) and $11.1 \pm 13 \mathrm{sNU}$ for the experiment using ${ }^{71} \mathrm{Ga}$. (See Fig. III-3.) These results suggest that further work is needed on the measurement and calculation of the input physics for a solar model to reduce the error in the ${ }^{37} \mathrm{Cl}$ capture rate prediction before any conclusions can be drawn abnut the solar neutrino problem, However, if the ${ }^{11} \mathrm{Ga}$ experiment is carried out, with its smaller calculational uncertainty ( $10 \%$ ), and a discrepancy between theory and experiment persists, it would be a strong signal of a problem with our understanding of solar and/or neutrino physics.

b. The ${ }^{7} L_{2}$ id, p) ${ }^{8} L_{i}$ Reaction Cross Section Near $0.78 \mathrm{MeV}$

A. J. Elwyn, R. E. Holland, C. N. Davids, and W. Ray, Jr. The total cross section for the ${ }^{7} \mathrm{Li}(\mathrm{d}, \mathrm{p})^{8} \mathrm{Li}$ reaction has been obtained at four energies near $0.78 \mathrm{MeV}$ from measurements, by time-of-flight techniques, of the differential cross sections for the outgoing protons at lab angles between $20^{\circ}$ and $155^{\circ}$. This cross section is used as normalization in the determination of the rate of the ${ }^{7} \mathrm{Be}(\mathrm{p}, \gamma)$ reaction which, as a link in the proton-proton chain of nuclear reactions that take place in the sun, is of prime importance to the calculation of the solar neutrino capture rate expected in the ${ }^{37} \mathrm{Cl}$ neutrino-capture experiments.

The result for the total cross section, $146 \pm 13 \mathrm{mb}$, is about $20 \%$ lower than the weighted mean of all previous absolute measurements, which differ from each other by as much as $40 \%$. It does, however, agree with a new measurement of the cross section recently obtained at Argonne in an experiment (described in the rep $\mathrm{rt}$ immediately following) in which the ${ }^{8} \mathrm{Be}$ 


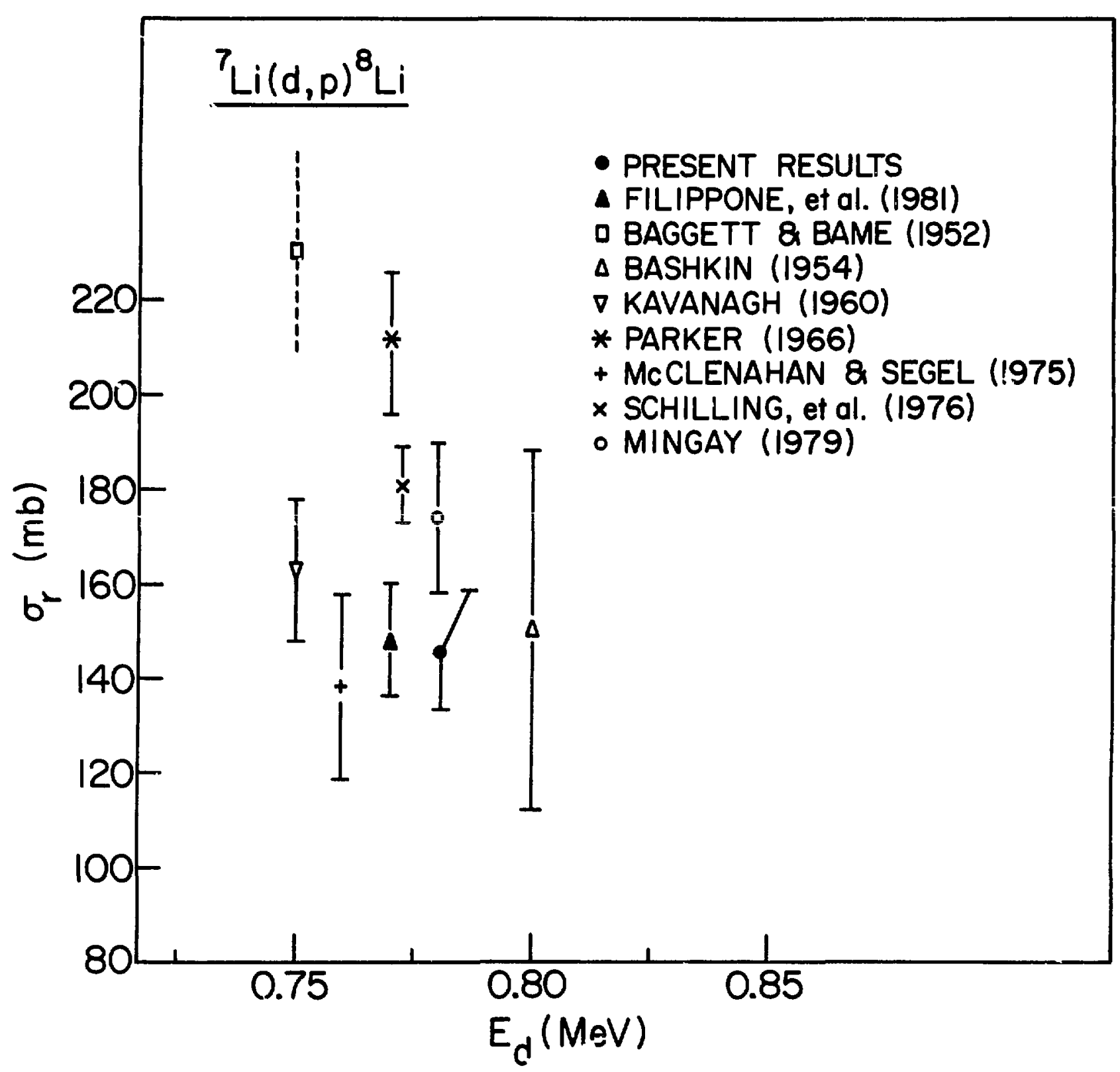

Fig. III-4. The total cross section for the ${ }^{7} \mathrm{Li}(\mathrm{d}, \mathrm{p})^{8} \mathrm{Li}$ (g.s.) reaction at energies near $0.78 \mathrm{MeV}$. The present average value along with that from the Argonne measurements of the breakup alpha particles are shown as solid circles and triangles, respectively. Previous measurements are also shown. 
breakup alpha particles from the ${ }^{8}{ }_{\text {L1 }}$ beta decay were detected. Figure III-4 compares the results of the Argonne measurements with previous experiments. If the present ${ }^{7} \mathrm{Li}(\mathrm{d}, \mathrm{p})$ cross section is used to scale previously measured ${ }^{7} \mathrm{Be}(\mathrm{p}, \gamma)$ values, the predicted solar-neutrino capture rate is reduced by $\geq 1$ solar neutrino unit from previously predicted rates.

c. The ${ }^{7} \mathrm{Li}(\mathrm{d}, \mathrm{p})^{8}{ }_{\mathrm{Li}}$ Reaction

B. Filippone, ${ }^{*}$ A. J. Elwyn, D. Koetke, ${ }^{\dagger}$ and W. Ray, Jr.

A measurament of the absolute cross section for the ${ }^{7} \mathrm{Li}(\mathrm{d}, \mathrm{p}){ }^{8} \mathrm{Li}$ reaction by dotecting the delayed $\alpha$ particles from ${ }^{8} \mathrm{Li}$ decay has been completed. ${ }^{1}$ Tiue value of this cross section at the peak of the $0.7 j-\mathrm{MeV}$ resonance has been used as the norma?izing reaction for most of the previous measurements of the ${ }^{7} \mathrm{Be}(\mathrm{p}, \gamma)^{8} \mathrm{~B}$ reaction mentioned above.

The cross section at the $0.77 \cdot \mathrm{Me} / \mathrm{J}$ resonance has been measured to be $148 \pm 12 \mathrm{mb}$. This new value for the cross section lowers the preaicted neutrino capture rate in the ${ }^{37} \mathrm{Cl}$ experiment by about $15 \%$ when used to normalize the ${ }^{7} \mathrm{Be}(\mathrm{p}, \gamma)^{8} \mathrm{~B}$ reaction. Figure III- 5 shows the effect of changes in the ${ }^{7} \mathrm{Be}(\mathrm{p}, \gamma){ }_{\mathrm{B}}^{8} \mathrm{~S}$ factor on the calculated ${ }^{37} \mathrm{Cl}$ solar ceutrino capture rate; the vertical line indicates the value inferred from the new measurement of the ${ }^{7} \mathrm{Li}(\mathrm{d}, \mathrm{p})^{8}{ }_{\mathrm{Li}}$ cross section.

* Thesis student, University of Chicago, Chicagc, Illinois.

† Valparaiso University, Valparaiso, Indiana.

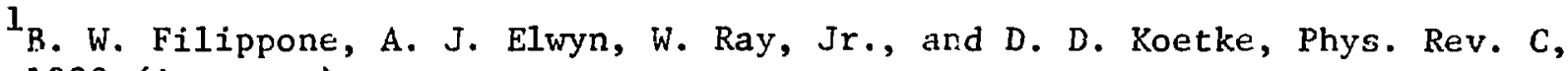
May 1982 (in press).

d. The ${ }^{7} \mathrm{Be}(\mathrm{p}, \gamma)^{8}$ Be Reaction

B. Filippone, , A. J. Elwyn, D. Koetke, ${ }^{\dagger}$ and W. Ray, Jr.

The development of a ${ }^{7} \mathrm{Be}\left(t_{1 / 2}=53\right.$ days $)$ target is under way in order to measure the ${ }^{7} \mathrm{Be}(\mathrm{p}, \mathrm{y})^{8} \mathrm{~B}$ cross section at low energies. It is this reaction in the sun that leads directly to the production of high-energy neutrinos from ${ }^{8}$ Be decay which comprise $280 \%$ of the neutrino capture rate in the ${ }^{37} \mathrm{Cl}$ solar neutrino experiment.

*Thesis student, University of Chicago, Chicago, Illinois.

${ }^{\dagger}$ Valparaiso University, Valparaiso, Indiana. 


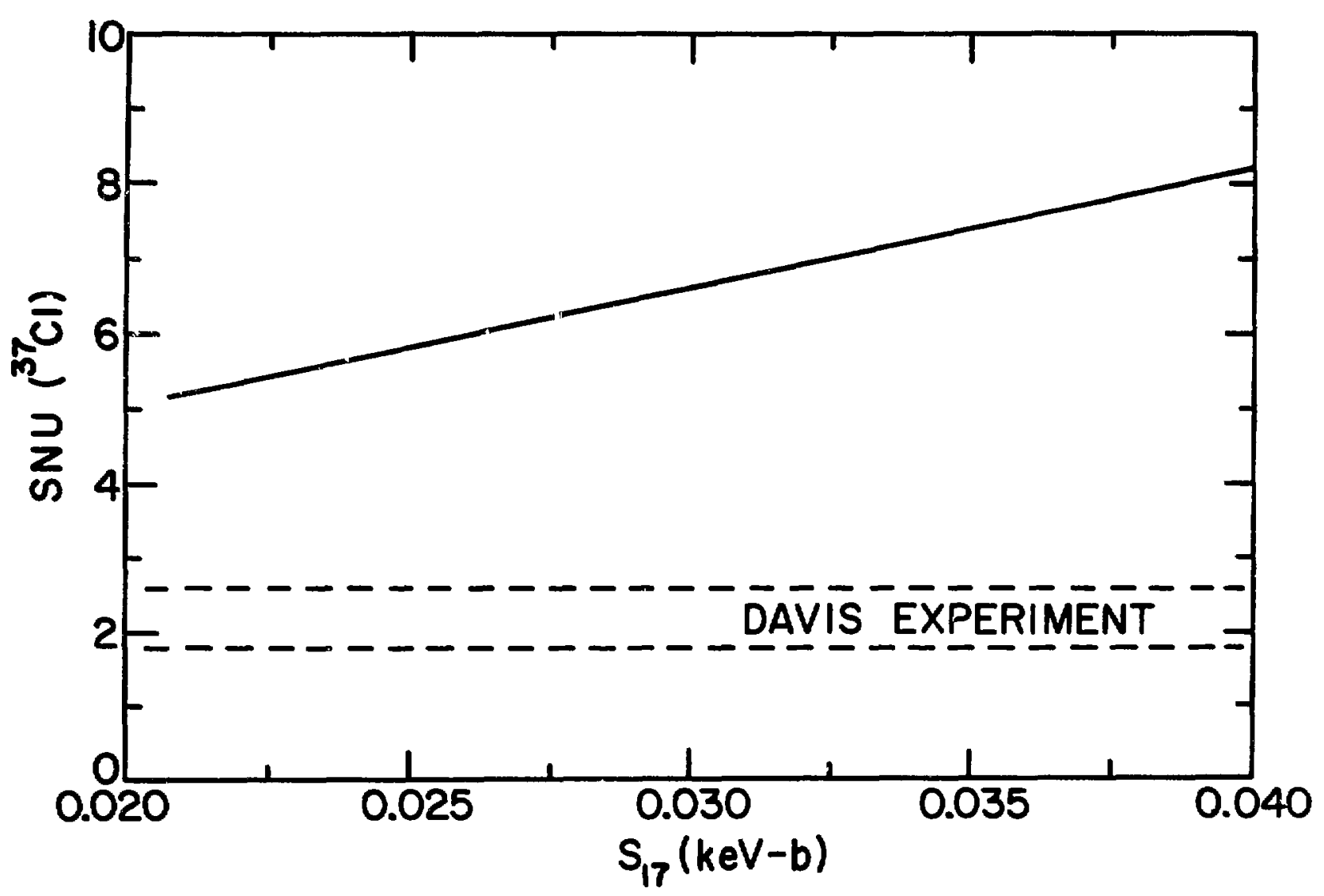

Fig. III-5. Dependence of the solar neutrino capture for a ${ }^{37} \mathrm{Cl}$ detector on the $7_{\mathrm{Be}}(p, \gamma){ }_{\mathrm{B}} \mathrm{S}$ factor. Also shown is the $(1-\sigma)$ range from the underground neutrino detector of $\mathrm{R}$. Davis, Jr., in Proceedings Informal Conference on Status and Future of Solar Neutrino Research, edited by G. Friedlander (Brookhaven Natione1 Laboratory, Upton, N.Y., 1978), BNL Report BNL $50879 \underline{1}, 1$. 
A preliminary target $(20.5 \mathrm{mC} 1$ ) homogeneous to better than $10 \%$, has been produced by a high voltage electroplating technique. The $7_{\mathrm{Be}}$ is first produced from the ${ }^{7} \mathrm{Li}(\mathrm{p}, \mathrm{n}){ }^{7} \mathrm{Be}$ reaction and then 1 s chemically separated from the Li. Following the plating of the ${ }^{7}$ Be onto a thin Pt backing, it is ignited to the oxide to form the refractory BeO. A Pb collimated Ge(Li; detector allows a measurement of the uniformity of the target.

Work is continuing on making a target with a much higher ${ }^{7}$ Be content ( $\sim 50 \mathrm{mCi}$ ) to permit measurement of the ${ }^{7} \mathrm{Be}(\mathrm{p}, \gamma)^{8} \mathrm{~B}$ cross section down to low energies.

e. Study of Nonresonant Capture in the ${ }^{27} \mathrm{Al}(\mathrm{p}, \gamma)$ and ${ }^{19} \mathrm{~F}(\mathrm{p}, \gamma)$ Reactions at A. J. Elwyn, G. Hardie, ${ }^{*}$ R. E. Segel, ${ }^{\dagger}$ M. Weischer, ${ }^{\dagger}$ E. Zupranska, ${ }^{\dagger}$ and W. Ray, Jr.

Using high beam currents ( 2100 pamps) and both $\mathrm{Ge}(\mathrm{Li}$ ) and NaI detectors, we are studying the nonresonant $\gamma$-ray yield in radiative capture reactions on ${ }^{27} \mathrm{Al}$ and ${ }^{19} \mathrm{~F}$ targets at incident energies near and below $1 \mathrm{MeV}$. In this region, nonresonant processes are expected to arise predominantly from a direct-capture mechanism where the incident projectile radiates a photon and enters a shell-model orbit of the target nucleus. Both reactions are of astrophysical interest in that measured cross sections should more clearly define the role of side branches in various nucleosynthesis processes. For ${ }^{27} \mathrm{Al}(\mathrm{p}, \gamma)$, we have identified $\gamma$-ray transitions consistent with the direct-capture process to both the ground and $1.78-\mathrm{MeV}$ state in ${ }^{28} \mathrm{Si}$. An excitation function for such transitions has been obtained between 0.8 and $1.8 \mathrm{MeV}$. Unfortunately the existence of a large number of narrow resonances in this reaction makes the interpretation of the observed "nonresonant" yield somewhat uncertain. In the ${ }^{19} F(p, \gamma)$ reaction, direct-capture transitions to the $1.634-\mathrm{MeV}$ state in ${ }^{20} \mathrm{Ne}$ are observed between incident energies from 0.6 to $1.2 \mathrm{MeV}$. By improving our techniques, particularly in the reduction of pileup due to the very intense yleld of $6-7-\mathrm{MeV} \gamma$ rays from the ${ }^{19} \mathrm{~F}(\mathrm{p}, \alpha \gamma)$ reaction, we hope to investigate nonresonant processes at incident energies well below $0.5 \mathrm{MeV}$. These experiments will continue through the present year.

\footnotetext{
*Western Michigan University, Kalamazoo, Michigan.

$t_{N}$ Nor thwestern University, Evanston, Illinois.

tOhio State University, columbus, Ohio.
} 


\section{SEARCHES FOR EXOTIC PARTICLES}

\section{A Cryogenic Search for $+1 / 3$ e Stable Charges}

D. Frekers, W. Henning, W. Kutschera, J. P. Schiffer, K. W. Shepard, C. Curtis, ${ }^{*}$ and C. Schmidt ${ }^{*}$

The results of experiments from Stanford University continue to report charges differing from integral multiples of the electron charge by $\pm 1 / 3 e$. The possibility that low temperatures (unique to the stanford experiments) may play a special role in stabilizing the diffusion of hypothetical $+1 / 3 e$ charged particles, cannot be ruled out completely. An experiment is planned for the summer of 1982 in which a cold $\left(\sim 4^{\circ} \mathrm{K}\right) \mathrm{Nb}$ filament will be placed in the terminal of an electrostatic accelerator and warmed up, while detectors will observe any charged particles that may be released from the filament. A negative result would help close a conceivable loophole in present experimerts.

*Fermi National Accelfatur Laboratory, Batavia, Illinois. 


\title{
IV. NEUTRON AND PHOTONUCLEAR PHYSICS
}

\author{
INTRODUCTION
}

A long-standing goal of neutron physics has been to provide measurements of the electric dipole moment of the neutron. At Argonne an experiment has been undertaken in order to measure the electric dipole moment of the neutron. The immediate goal of this program is to attain a sensitivity of approximately $10^{-25} \mathrm{e}-\mathrm{cm}$. A result of this accuracy would provide a test of recent gauge theories of weak interactions, and in particular, the Weinberg-Salam standard model.

The photoneutron method permits the study of a broad range of nuclear phenomena which is inaccessible by other reactions or even neutron induced reactions. The photoneutron method has been successfully applied to the study of collective dipole excitations as well as single-particle resonances in nuclei. During the past year, the photoneutron method has been exploited in order to study the basic nucleon-nucleon interaction and the associated meson exchange currents. With the extremely high electron currents and the unique beam transport system available at the ANL photoneutron facility, the deuteron photodisintegration reaction is being studied with substantially greater accuracy than has been previously available. In particular, the relative angular distribution of neutrons from the $2 \mathrm{H}(\gamma, n) \mathrm{H}$ reaction has been measured with high accuracy. Photoneutron polarization measurements from this process are in progress. 


\section{A. NEUTRON RESEARCH}

\section{Measurement of the Electric Dipole Moment of the Neutron}

V. E. Krohn, G. R. Ringo, T. W. Dombeck, M. S. Freedman, J. M. Carpenter, ${ }^{*}$ and J. W. Lynn ${ }^{\dagger}$

The purpose of this project is to measure the electric dipole moment (EDM) of the neutron. Because this can be done by a measurement of frequency change, it can be done with great sensitivity and indeed is generally believed to be the most sensitive test available of time-reversal invariance. The present situation is that with about a factor of 10 improvement in sensitivity, a whole class of gauge theories-those which explain CP failure by introducing a new scalar field [e.g., S. Weinberg, Phys. Rev. Lett. 37,657 (1976)]-can be given a definitive test.

Since the measurement of the neutron EDM is fundamentally a frequency measurement, its statistical uncertainty is inversely proportional to the duration of the measurement. It is therefore natural to try the measurement on ultracold neutrons (UCN). These neutrons of $v<7 \mathrm{~m} / \mathrm{s}$ can be kept in a bottle for hundreds of seconds. We propose to do this using two unique features. First, we propose to use a pulsed neutron source and keep the inlet to the bottle open only when the pulsed source is on, thus allowing a buildup to an asymptotic density determined by the peak flux of the source instead of the average, This has the advantage that pulsed sources have peak fluxes that are much higher than the average fluxes of steady state sources of the same average power. Second, we propose to produce the UCN by Bragg reflection of considerably faster $(400 \mathrm{~m} / \mathrm{s}$ vs $7 \mathrm{~m} / \mathrm{s}$ ) neutrons from a moving mica crystal designed so that the reflected neutrons are almost stationary in the laboratory system. The advantage of this is that it avoids the problems of extracting the very delicate $U C N$ from the hard to control environment in a high flux source.

The present state of the project is that both of these ideas have been tested and shown to be practical as have several other ideas for enhancing the production of UCN, such as the use of reflectors around the moving crystal and funnels to concentrate the UCN in real space at the expense of

*Intense Pulsed Neutron Source, ANL.

${ }^{\dagger}$ University of Maryland, College Park, Maryland. 
their concentration in velocity space. The experiment has been serfously delayed by the delay in operating the liquid hydrogen moderators in IPNS. However, a greatly improved bottle has been constructed, large enough to be considered for use as a reservoir in the EDM experiment, and work on important auxiliary tests, such as the reduction of electrical leakage currents in the measurement bottle, is going forward. We have explored the possibilities of doing the experiment at another neutron source and both the WNR at Los Alamos and the research reactor at Brookhaven appear to be possible sites that might offer some advantages.

The next stage of the project is to test the bottle we have built. Then we must build a suitable bottle in a comblned magnetic and electric field and measure the effect of reversing the electric field on the precession rate. 


\section{B. PHOTONUCLEAR PHYSICS}

\section{a. Photodisintegration of the Deuteron}

R. E. Holland, R. J. Holt, H. E. Jackson, R. D. McKeown, * J. R. Specht, and $K$. Stephenson

Deuteron photodissociation provides the simplest process for the study of meson exchange and virtual isobar effects. Theoretical predictions of the polarization of the ejected nucleons indicate a high degree of sensitivity to these non-nucleonic effects in the nucleus. Furthermore, discrepancies between the observed cross section for the $D(\gamma, n) H$ reaction and theoretical predictions have led to doubt in recent years about the understanding of this simple reaction. For these reasons, our objectives are to provide high accuracy relative angular distributions as well as photoneutron polarization data.

\section{(i) Angular Distributions}

The relative angular distributions at $45^{\circ}, 90^{\circ}, 135^{\circ}$, and $155^{\circ}$ have been measured from $E_{\gamma}=3.5$ to $19 \mathrm{MeV}$ for the $D(\gamma, n)$ reaction. The error limits are of the order of 2 to $3 \%$. The data were found to be particularly sensitive to the E1-E2 interference term in the differential cross section. Although the analysis is still underway, it appears that the theoretical calculations will be in disagreement with the results. The angular distributions should be completed during this year.

\section{(ii) The $D(\gamma, \vec{n}) H$ Reaction}

A measurement of the photoneutron polarization for the $D(\gamma, \vec{n})$ reaction was performed at an angle of $90^{\circ}$. A neutron polarimeter which employed a natural carbon analyzer was used for this measurement. The polarimeter was calibrated in place with a neutron double-scattering method. These preliminary results indicate a surprising trend in the polarization. The results at $90^{\circ}$ are shown in FIg. IV-1. The solid points correspond to the present work, while the hatched area refers to the work of Nath et al. ${ }^{1}$ The data are compared to the impulse calculation of Partovi ${ }^{2}$ (solid curve) and the calculation, which includes meson exchange currents, of Hadjimichael ${ }^{3}$

*University of California, Pasadena, California.

${ }^{1}$. Nath, F. W. K. Firk, and H. L. Schultz, Nucl. Phys. Al94, 49 (1972).

2F. Partovi, Ann. Phys. 27, 79 (1964).

3E. Hadjimichae1, Phys. Lett. 46B, 147 (1973). 


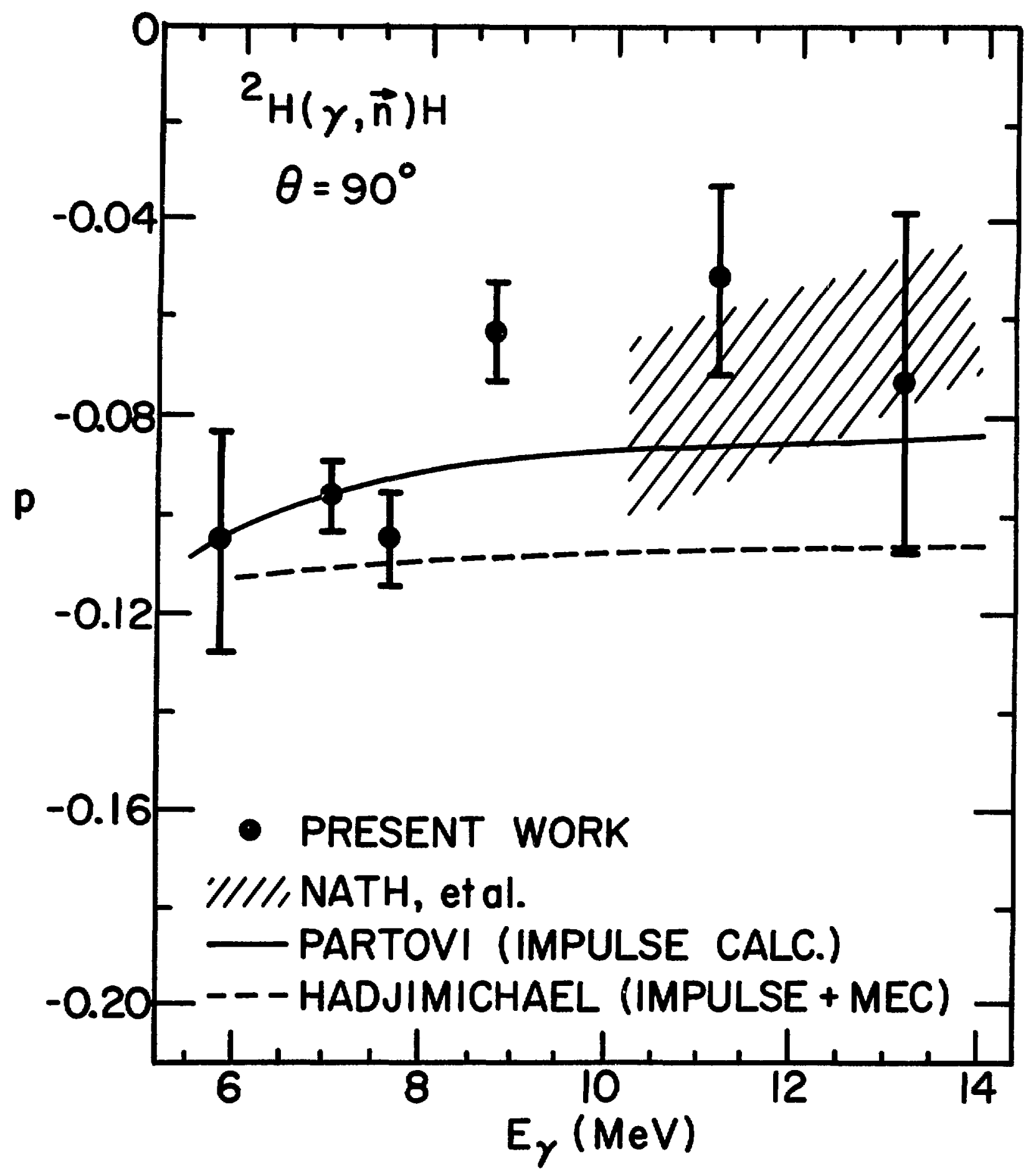

Fig. IV-1. The data points refer to the present work. The hatched region refers to the results of Nath et al. The solid curve indicates the Impulse calculation of Partovi, while the dashed curve includes meson exchange currents. 
(dashed curve). Clearly, the inclusion of meson exchange currents worsens the agreement with the present work. Since the photoneutron polarization at $90^{\circ}$ is primarily due to the interference of $\mathrm{El}$ and $\mathrm{Ml}$ amplitudes, the present work suggests that the calculated $M l$ contribution is too large in this energy region. As a further study of this problem, measurements of the photoneutron polarization at an angle of $135^{\circ}$ are underway.

\section{b. Isospin Splitting of the Giant Dipole Resonance in ${ }^{60} \mathrm{N1}$}

R. J. Holt, H. E. Jackson, R. D. McKeown, ${ }^{\star}$ A. M. Nathan, ${ }^{\dagger}$ and J. R. Specht

The photoneutron reaction is a selective probe for isospin splitting. For example, in a $\left(\gamma, n_{0}\right)$ reaction only photoneutrons from the $T_{<}$component are allowed by the isospin selection rules; whereas in $\left(\gamma, p_{0}\right)$ reactions, both components can be excited.

The cross section at $90^{\circ}$ for the ${ }^{60} \mathrm{Ni}\left(\gamma, \mathrm{n}_{0}\right){ }^{59} \mathrm{Ni}$ reaction was measured between 14 and $20 \mathrm{MeV}$ using the ANL Photoneutron Facility. There are clear isospin dependent effects in the cross section. There are two distinct peaks in the $\left(\gamma, \mathrm{P}_{0}\right)$ data of Diener et al. ${ }^{1}$ and only one in $\left(\gamma, n_{0}\right)$. This is consistent with the isospin-splitting model of Fallieros and Goulard. ${ }^{2}$ The relative strengths of the two components of the giant dipole resonance were deduced from the elastic photon scattering measurements for ${ }^{60} \mathrm{Ni}$.

*University of California, Pasadena, California.

${ }^{\dagger}$ University of Illinois, Urbana, Illinois.

$1_{E}$. M. Diener, J. F. Amann, P. Paul, and S. L. Blatt, Phys. Rev. C $\underline{3}$, 2303 (1971).

2. Fallieros and B. Goulard, Nucl. Phys. A147, 543 (1970). 


\title{
V. THEORETICAL NUCLEAR PHYSICS
}

\author{
INTRODUCTION
}

The nuclear theory program is almed at understanding the projertles of nuclel and their behavior in interaction with probes of strong, electromagnetic or weak nature. Some of the interpretations arise from phenomenological models. Other work concentrates on developing the consequences of nucleon-nucleon forces and the effects of including subnucleon degrees of freedom. The pion plays an important role in our program, both as a probe and as the dominant component of the problem of the nuclear force in nucle1. Our main areas of concentration are:

1. Heavy-ion direct reactions at nonrelativistic energies.

2. Nuclear shell theory and nuclear structure.

3. Nuclear forces and subnucleon degrees of freedom.

4. Intermediate-energy physics with pions, electrons and nucleons.

5. High-energy heavy ion collisions.

Strong emphasis is placed on comparing the results of theoretical studies with experimental observations and in developing comprehensive theorles which can be applied to a wide range of problems. Developments in the main areas are described in the following sections. 


\section{A. HEAVY-ION DIRECT-REACTION THEORY}

S. C. Pleper, M. H. Macfarlane, ${ }^{*}$ M. J. Rhoades-Brown, ${ }^{\dagger}$ and others

Heavy-ion scattering at energles fust above the Coulomb barrier typically exhibits large cross sections to several low-lying states (Inelastic scattering) and smaller cross sections to many states reached by few-nucleon. transfer reactions. Attempts to predict these cross sections (both inelastic and transfer) with the distorted wave Born approximation (DWBA) have of ten been unsuccessful, suggesting the necessity of coupled-channels calculations. Such calculations including all of the transfer channels are clearly not yet possible. However the very fact that there are so many particle-transfer channels and the small cross sections to each one suggest that the effects of these channels on the elastic and Inelastic scattering wavefunctions could be accounted for by the 1maginary part of the optical potential, although a different shape from the Woods-Saxon forms conventionally used may be needed. The few strongly populated inelastic channels could have important explicit effects that are not naturally accounted for by a complex optical potential.

For this reason we have concentrated on developing a program, Ptolemy, for coupled-channels calculations of inelastic scattering. During the last year we have been making such calculations at energies from below the Coulomb barrier to several times the Coulomb barrier, but a detalled comparison with data has not yet been made. If these calculations do accurately reproduce the inelastic scattering data, then the coupled-channels Born approximation may be adequate for the transfer reactions.

Heavy-ion inelastic scattering should be a useful tool for extracting nuclear-structure information about low-lying collective states. In such scattering both Coulomb and nuclear excitation are important and the interference between these two processes can also have measurable effects and these terms can distinguish between different models (rotational, vibrational, coexistence) for a given state.

\footnotetext{
*Indiana University, Bloomington, Indiana.

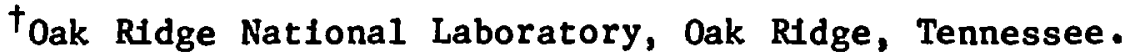

\section{a. Ptolemy}

Steven Pleper and M. J. Rhoades-Brown*

In 1980 Ptolemy was expanded to do coupled-channels calculations for Inelastic scattering. During 1981 a number of improvements and corrections were made to the coupled-channels part and the ability to use the deformedrotational model was added. The program will now automatically generate symmetrized coupling schemes for identical nuclel; this reduces by about $40 \%$

\footnotetext{
*Oak RIdge National Laboratory, Oak RIdge, Tennessee.
} 
the time needed for ${ }^{12} \mathrm{C}+{ }^{12} \mathrm{C}$ inelastic calculations. A further improvement Is the use of the epsilion algorithm to accelerate the convergence of the scattering amplitude. With this method, converged differentlal cross sections are achleved by summing only 10 or $20 \mathrm{~J}$ values past the total $\mathrm{J}$ corresponding to the classical deflection function; previously it was often necessary to sum several thousand terms beyond this value.

b. Optical Potentials for Coupled-Channels Calculations

S. C. Pieper and M. H. Macfarlane*

A few years ago trivially-equivalent local potentials (TELP) were proposed as a means of accounting for coupled channels effects in singlechannel optical-model calculations. Results were presented that showed that these potentlals reproduced well the coupled-channels effects of the Coulomb potential. Although the potentials were (and are) used in cases for which nuclear coupling is also important, their adequacy in such cases was not studied. We have compared TELP calculations with exact coupled-shannels calculations and find that above the Coulomb barrier the TELP is a poor approximation. This means that an optical potential fit to data using TELP does not reproduce the same data when a complete coupled-channels calculation is made.

In heavy-ion scatering experiments it is sometimes impossible to separate low-lying inelastic states from the ground state. In such cases one can only extract quasi-elastic differential cross sections, and optical potentials fit to these data have been published. We find that when these potentials are used in a coupled-channels calculation, and the resulting differential cross sections to ground and excited states are summed, the quasi-elastic data are not reproduced. That 1s, the channel coupling does not fust redistribute the flux at a given angle anongst the different channels, but rather changes the total flux going to the angle.

The present status of this study is that the only rellable way to produce a potential that reproduces data in a coupled-channels calculation is to make a coupled-channels fit. Since such calculations can be quite time consuming, we intend to continue looking for other possibilities.

\footnotetext{
*Indiana University, Bloomington, Indiana.
} 


\section{c. Sensitive Radius}

S. C. Pleper and M. H. Macfarlane*

In previous years we had demonstrated that the so-called "sensitive radius" that had been reported from optical-model fits to heavy-ion elastic scactering data is just an artifact of the limited functional forms used for the potentials. An article on this work was written and has been published.

\footnotetext{
*Indiana University, Bloomington, Indiana.
} 
B. NUCLEAR SHELL THEORY AND NUCLEAR STRUCTURE

D. Kurath, R. D. Lawson, and others

The main effort has been directed toward providing explanations for observations in current experiments. This was generally done by applying the nuclear shell model including residual interactions approprlate to a general region of nuclei. Observations from heavy ion reactions, inelastic scattering with electrons and pions as well an light ion reactions were treated. Studies were also made with the objective of seeing what aspects of nuclear structure are likely to be important for experiments with a high-intensity electron accelerator of several $\mathrm{GeV}$ in energy.

a. Senfortty Isomers Near $z=64$

R. D. Lawson

Experiments on ${ }_{66}^{148} \mathrm{Dy}_{82}$ and ${ }_{67}^{149} \mathrm{Ho}_{82}$ have shown that to good approximation $\mathrm{N}=82 \quad \mathrm{Z}=64$ acts as a doubly closed shell and that the protons outside the $z=64$ core are filling the $h_{11 / 2}$ single-particle orbit. If one takes the residual two-body $h_{11 / 2}$ interaction from the experimental spectrum of ${ }^{148} \mathrm{Dy}$, one can show that senfority is predicted to be conserved to a very high degree. ${ }^{1}$ When seniortty is conserved, the $B(E 2)$ values between seniority two states of the $\left(h_{11 / 2}\right)^{4}$ configuration should be $1 / 4$ the values observed for the analogous $\left(h_{11 / 2}\right)^{2}$ transitions and those in $\left(h_{11 / 2}\right)^{5}$ between seniority three states should be $1 / 9$ the analogous $\left(\mathrm{h}_{11 / 2}\right)^{3}$ decays. This means that in ${ }_{68}^{150} \mathrm{Er}_{82}$ and ${ }_{69}^{15 T^{2}} 82$ one shouid observe very long-lived states and one predicts $B\left(E 2 ; 10^{+} \rightarrow 8^{+}\right)=10.8 \mathrm{e}^{2} \mathrm{fm}^{4}$ in ${ }^{150} \mathrm{Er}$ and $B\left(E 2 ; 27 / 2^{-}+23 / 2^{-}\right)=9.22 \mathrm{e}^{2} \mathrm{fm}^{4}$ in ${ }^{151_{\mathrm{Tm}}}$. Experiments carried out at Argonne yleld $11.3 \pm 0.7 \mathrm{e}^{2} \mathrm{fm}^{4}$ and $11.7 \pm 1.3 \mathrm{e}^{2} \mathrm{fm}^{4}$, respectively, for these transitions in excellent agreement with the theoretical predictions.

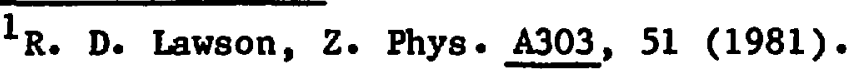

b. High Spin States in ${ }^{94} \mathrm{Ru}$ and ${ }^{95_{\mathrm{Rh}}}$

A. Amuga ${ }^{*}$ and R. D. Lawson

High spin states and some gamma decay lifetimes have recently been observed in ${ }_{44}^{94} \mathrm{Ru}_{50}$ and ${ }_{45} \mathrm{Rh}_{50}$. In order to check the consistency of the tentative spin assignments, we have carried out a shell-model calculation for

\footnotetext{
"University of Ife, Ile-Ife, Nigeria.
} 
the states of these nucle1. ${ }_{38}^{88} \mathrm{y}_{50}$ was assumed to be a doubly closed shell core and the six (seven) valence nucleons in ${ }_{44}^{44} \mathrm{Ru}_{50}$ and $95{ }_{45} \mathrm{Rh}_{50}$ were assumed to occupy the $2 \mathrm{p}_{1 / 2}$ and $1 \mathrm{~g}_{9 / 2}$ orbits. The residual two-body interaction was taken from the work of Gloeckner and Serduke. ${ }^{1}$ The shell-model results support the expirimental angular momentum assignments. In ${ }_{44}^{94} \mathrm{Ru}_{50}$ the negative parity states have the structure $\left[\left(g_{9 / 2}\right)_{J \nu}^{5} \times p_{1 / 2}\right]_{I}$, where $J$ and $\nu$ denote the angular momentum and seniority of the $\left(g_{9} / 2\right)^{5}$ configuration. Since E2 transitions between states of the same senfority vanish at the center of the shell, one might expect to see senfority 1somers. However, this 1somerism is not observed experimentally and our calculations, which in some instances predict a near degeneracy of the states described by $\left[\left(g_{9 / 2}\right)_{\mathrm{JV}=3}^{5} \times \mathrm{p}_{1 / 2}\right]_{\mathrm{I}}$ and $\left[(89 / 2){ }_{J V=5}^{5} \times p_{1 / 2}\right]_{I}$, provide an explanation for this.

1D. H. Gloeckner and F. J. D. Serduke, Nuc1. Phys. A220, 477 (1974).

c. Electron Scattering to $10^{-}$States in ${ }^{90} \mathrm{Zr}$

A. Amusa ${ }^{*}$ and R. D. Lawson

Inelastic electron scattering on ${ }_{40}^{90} \mathrm{Zr}_{50}$ populates a single $10^{-}$le rel at $7.36 \mathrm{MeV}$ with an intensity about $40 \%$ of that expected for a pure netritron $\left(h_{11 / 2} 8_{9 / 2}^{-1}\right)$ particle-hole excitation. Since it is known that the valence protons in the ${ }^{90} \mathrm{Zr}$ ground state occupy both the $2 \mathrm{p}_{1 / 2}$ and $18_{9 / 2}$ states, the transition ce.ri also involve proton excitation. In order to see how this affects the predicted strength, we have made a shell-model calculation for the $10^{-}$states in $90 \mathrm{Zr}$ assuming $\mathrm{N}=\mathrm{Z}=38$ is an inert core. The two valence protons and ten valence neutrons are restricted to the $2 \mathrm{p}_{1 / 2}, 1 g_{9 / 2}$ and $1 \mathrm{~h}_{11 / 2}$ levels with at most one nucleon in $h_{11 / 2}$. Wichin this model space there are $3010^{-}$ states. When the $\left(2 \mathrm{p}_{1 / 2}, 1 \mathrm{~g}_{9 / 2}\right)$ interaction is taken from a previous $\mathrm{N}=49$ study and the force Involving an $h_{11 / 2}$ particle is taken to be the SchifferTrue potential, the predicted strength to the yrast $10^{-}$level drops to about $58 \%$ of the pure neutron $\left(h_{11 / 2}, 89 / 2\right)$ value. There is only one other state that should be appreclably populated and that is the second $10^{-}$predicted to $11 e$ about $650 \mathrm{keV}$ above the yrast one. The theoretical transition strength is a factor of three smaller than that for the yrast level and would make the state very difficult to see experimentally.

\footnotetext{
*University of Ife, Ile-Ife, Nigeria.
} 


\section{d. Electron Scattering on ${ }^{6} \mathrm{L1}$}

\section{R. D. Lawson}

Electron scattering experiments on ${ }^{6} \mathrm{LI}$ heve been carried out and the $M 1$ form factor for excitation of the $3.56-\mathrm{MeV} I=0^{+} \mathrm{T}=1$ state has been obtained. This form factor is found to decrease at high momentum transfer somewhat more slowly than 18 predicted by the shell model. We have investigated the effects of short-range correlations on this excitation by assuming that the usual uncorrelated $\mathrm{p}$-shell wave function is multiplied by a factor $\left\{1-\exp -\beta^{2}\left(r_{1}-r_{2}\right)^{2}\right\}$, where $r_{1}$ and $r_{2}$ are the coordinates of the two valence nucleons and $\beta=1 \mathrm{fm}^{-1}$. These short-range correlations have been found to have a negligible effect on the form factor for the transition.

e. Effects of the $\triangle$ Resonance on $M 1$ Properties of ${ }^{17_{0}}$

\section{R. D. Lawson}

The measured magnetic moment of the ground state of ${ }^{17_{0}}$ is $\mu=-1.8937 \mathrm{~nm}$, which is quite close to the expected Schmidt value of -1.9131 $\mathrm{nm}$. On the other hand, the experimental $B(M 1)$ governing the $M 1$ transition between the $5.08 \mathrm{MeV} 3 / 2^{+}$level and $5 / 2^{+}$ground state is a factor of three smaller than the single-particle estimate. We have investigated the effect of the $\Delta$ resonance on these two properties. The $\Delta-m i x i n g$ amplitude was calculated in first-order perturbation theory using the $\Delta$-nucleon potential suggested by Smith and Pandharipande. It was found that the admixtures have 1ittle effect on the $d_{5 / 2}$ magnetic moment but do decrease the $B(M 1)$ significantly.

\section{f. Strong Ml Decays in ${ }^{12} \mathrm{C}$}

D. Kurath

Gamma decays have recently been studied via ${ }^{11} B(p, \gamma)^{12} C$ for two resonances near $18 \mathrm{MeV}$ of excitation in ${ }^{12} \mathrm{C}$. The dominant modes of decay are strong MI transitions from the $\left(0^{+}, 1\right)$ resonance at $17.77 \mathrm{MeV}$ to the $\left(1^{+}, 0\right)$ state at $12.71 \mathrm{MeV}$ and from the $\left(3^{-}, 1\right)$ resonance at $18.36 \mathrm{MeV}$ to the $\left(3^{-}, 0\right)$ state at $9.64 \mathrm{MeV}$. Shell model calculations of these transition probabilities have been carried out for comparison with observations. The measured $\left(0^{+}, 1\right)$ to $\left(1^{+}, 0\right)$ transition 18 strong with $B_{M I}$ equal to $90 \%$ of the well-known $B_{\mathrm{MI}}(00+11)$ from the ${ }^{12} \mathrm{C}$ ground state to the $15-\mathrm{MeV}$ level. Even 80 , the value 
calculated in a $(1 p)^{8}$ space is considerably larger, suggesting that the observed $\left(0^{+}, 1\right)$ level contains $(1 p)^{6}(s d)^{2}$ components, an explanation that is also consistent with transfer data. The measured $\left(3^{-}, 1\right)$ to $\left(3^{-}, 0\right)$ transition

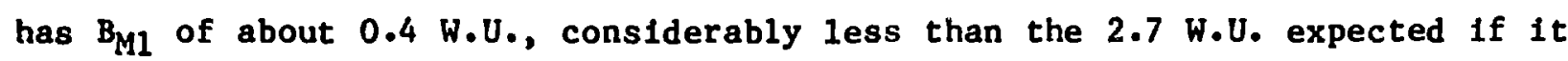
were a simple analog to anti-analog transition based on the $A=11$ ground state. The shell model glves $B_{M 1}$ in agreement with the measured value and also gives the observed value for the $E 1$ branch to the $\left(2^{+}, 0\right)$ state. A joint paper of measured and calculated results has been published. 1

1's. S. Hanna, W. Feldman, M. Suffert, and D. Kurath, Phys. Rev. C 25, 1179 (1982).

\section{g. Distribution of Gamow-Teller Strength}

D. Kurath

The distribution of Gamow-Teller strength is obtained by interpreting results of $(p, n)$ measurements at $0^{\circ}$ with protons of about $200 \mathrm{Me} \%$. A general feature seems to be that the total strength observed is considerably less than is expected from nuclear models. Therefore the distribution of G-T strength has been calculated with the shell model for targets in the lp shell in order to compare with experiment. A comparison for ${ }^{14} \mathrm{C}$ shows that only about $60 \%$ of the calculated strength is observed. On the other hand, in ${ }^{12} \mathrm{C}$ nearly all the calculated strength is seen. A detailed comparison as a function of mass number $A$ is relevant for explanations of GT suppression such as the proposed effect of admixture of $\Delta$ excitation.

\section{h. Analysis of Inelastic Scattering of Plons}

\section{T.-S. H. Lee and D. Kurath}

Experiments have been carried out by the Argonne users at LAMPF to study Inelastic scattering of $\pi^{ \pm}$at energles near the $(3,3)$ resonance on targets $10_{\mathrm{B}}, \mathrm{i1} \mathrm{B}$ and $14 \mathrm{~N}$. Experience with our DWA code for calculating cross sections to particular final states has shown that certain multipoles, $J(L S)$, of the particle-hole transition density dominate in producing strong transitions. Here $J, L$ and $S$ are, respectively, the total orbital and spin angular momentum. Good approximate cross sections for a given target are obtained by first running the DWIA calculation for each multipole with unit nuclear amplitude as Input and storing the resultant cross section; the 
calculated cross sections for a particular excited state are then obtalned by weighting the cross section for each $J$ by the particular transition amplitude. In this way the spectrum of strong states for a given nucleus can be computed with much reduced time and cost since the weighting of the few DWIA cross sections and graphing of results is done very efficlently with a Speakeasy program. The analysis of the ${ }^{10} \mathrm{~B}, 11_{\mathrm{B}}$ and $14_{\mathrm{N}}$ experiments is being prepared for publication.

In a recent experiment on ${ }^{14} \mathrm{C}$ striking differences were found for the relative cross sections in $\pi^{+}$and $\pi^{-}$excitation of the two $2^{+}$states at 7.0 and $8.3 \mathrm{MeV}$. It is known that these are strong mixtures of the lower $2^{+}$state In the $(1 p)^{10}$ space and a $(1 p)^{8}(2 s d)^{2}$ state that is essentla1ly the ${ }^{12} \mathrm{C}$ ground state coupled to the $(2 \mathrm{sd})^{2}$ part of ${ }^{18} \mathrm{o}$. A shell-model calculation gives fust such a description and gives a qualitative explanation of the observations. 


\title{
C. NUCLEAR FORCES AND SUBNUCLEON DEGREES OF FREEDOM
}

\author{
F. Coester, B. D. Day, T.-S. H. Lee, J. Parmentola, R. Wiringa, \\ and collaborators from other institutions
}

Much of our work has been motivated by two central questions: What should be the active degrees of freedom in the many-body theory of nucle1? (2) What is the Hamiltonian that governs the nuclear many-body dynamics?

Conventional nuclear theory assumes that nucleons are the active degrees of freedom and other degrees of freedom are burled in the Hamiltonian. Accurate calculations of key properties of simple systems play a key role in the determination of the interactions. Work establishing that two-body forces cannot account for the propertles of nuclear matter came to a conclusion in 1981. During 1981 we began a systematic investigation of the effects of realistic three-body forces in 3- and 4-body nuclel, nuclear matter and neutron stars. These effects are in general not approximated by density dependent two-body forces.

At Intermediate energles plons and nucleon lsobars should be included among the active degrees of freedom. We have created a crude relativistic model which includes $N \Delta$ transition petential, pion production and absorption. The numerical results are encouraging. A more realistic implementation of the same 1deas is in progress. For many purposes the effects of $\Delta$ decay are negligible. Inclusion of a stable $\Delta$ can be expected to account for an important part of the three-body force effects in nuclei, and may produce effects not readily reproduced by a nucleon Hamiltonian with small many-body forces. A realistic two-body interaction involving stable $\Delta$ 's is under construction. We plan to include $\Delta$ decay by modifying this potential.

There is, at present, no credible way to include quark degrees of freedom among the active dynamical vartables in nuclear dynamics. Knowledge about the quark structures of nucleons can and should, of course, influence the construction of acceptable Hamiltonians. The chiral bag model could be a basis for multinucleon dynamics. But an accurate solution of the one-nucleon problem is a nontrivial prerequisite to progress in this direction. Good approximations are avallable for large radil and small couplirg constants and for small radil and large coupling constants. We are investigating the qualitative features and the range of validity of the strong coupling approximation.

There are multiple reasons why the nonrelativistic formulation of nuclear dynamics is inadequate: Relativistic effects in nuclear structure are small but not necessarily negligible. Models in which the sum of the particle masses is not conserved cannot be Galilean 1nvartant. Because of the small pion mass pion velocities are usually relativistic. A consistent calculation of electromagnetic form factors for large momentum transfer requires Lorentz invariant dynamics. We have established a general constructive framework for a relativistic dynamics of nucleons and mesons. 


\section{a. Nuclear Saturation from Two-Nucleon Potentials}

B. D. Day

To test a many-body Hamiltonian based on a two-body potential, it is Imperative to obtain an accurate approximate solution to the many-body Schrödinger equation for that Hamiltonian. A method to do this has been developed in previous work. It is based on a system of coupled equations for the amplitudes to excite 2,3, . . particle-hole palrs out of the Ferm1 sea (the coupled cluster equations). Tests of its accuracy include internal consistency checks and comparison with Monte-Carlo varlational results for model central potentlals. For an assumed two-body potential, the method gives the saturation point of nuclear matter with an accuracy of about $\pm 2 \mathrm{MeV}$ in energy per nucleon and $\pm 0.1 \mathrm{fm}^{-1}$ in Ferni momentum $k_{F}$. Since an arbitrary nonlocality in the potentlal can be handled, the method can treat potentials such as the Paris potentfal and the Bonn one-boson-exchange potential, which are at present beyond the reach of the variational method. Therefore, for the first time, one is in a position to answer the question of whether a twonucleon potential that is coni, tent with scattering data can quantitatively account for nuclear saturation. Calculated saturation points are shown in Fig. V-1. The solid circles are calculated fin lowest order, 1.e., including only 2-particle 2-hole excitation amplitudes, for a variety of nucleon-nucleon potentials. Our more accurate method has been applied to four potentials, and the resulting saturation points lie at the tips of the arrows. The potentials are Reid (MRSC), Bethe-Johnson (MRBJ), and Bonn and Paris. The oval gives the estimated calculational uncertainty in the Reid (MRSC) saturation point. All four calculated points lie far from the empirical saturation point, which lies inside the rectangle. The properties of the potential that are most relevant for saturation are the sirength of the tensor force and the strength of the short-range repulsion. The four potentlals treated cover a wide range of these strengths, as well as having several different types of nonlocality. Yet none of them come close to the empirical saturation point. We conclude that two-body potentlals that are fitted to scattering data cannot account for nuclear saturation. This work has been published in Phys. Rev. Lett. 47, 226 (1981). 


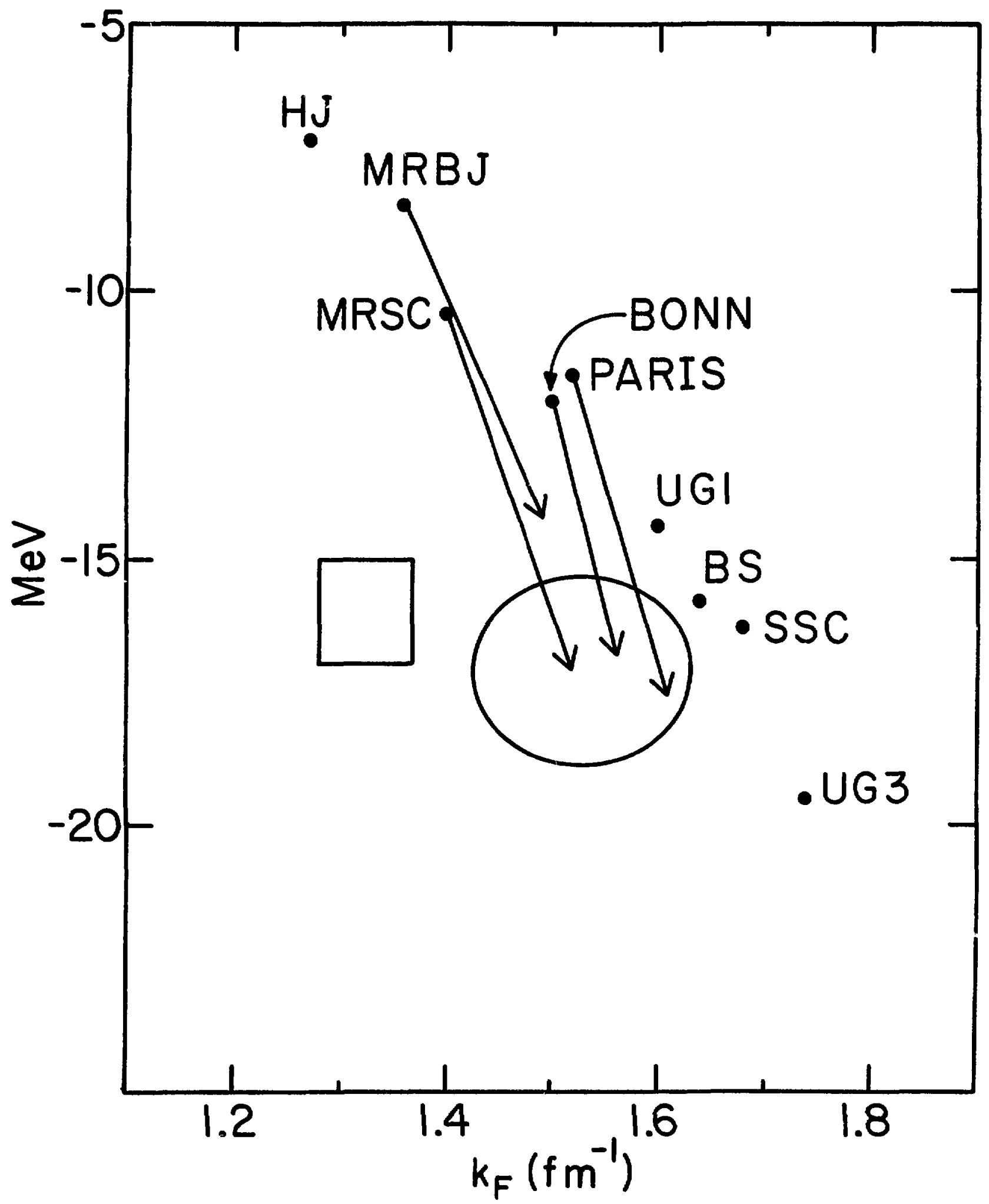

Fig. V-1. Calculated and empirical saturation points for nuclear matter. The empirical saturation point lies inside the small rectangle. 
b. Three-Body Forces in Light Nuclel and Nuclear Matter

R. Wiringa, J. Carlson, * and V. R. Pandharipande*

Realistic models of three-nucleon interaction (TNI) have been examined in ${ }^{3} \mathrm{H},{ }^{3} \mathrm{He}$, and ${ }^{4} \mathrm{He}$ nuclel, and in nuclear matter. Two-nucleon potentials that fit NN scattering data, such as Reid, Paris, and Urbana-v 14 , consistently underbind the light nuclei while overbinding nuclear matter at too high saturation density. The addition of TNI to the many-body Hamiltonian can significantly lessen this problem. The Tucson and 1sobar intermediatestate models of the two-pion-exchange potential $\left(V_{2 \pi 3 N}\right)$ were studied along with an intermediate-range three-nucleon iepulsion $\left(V_{3 N R}\right)$. The Urbana- $v_{14}$ model was used for the two-body force, and variational upper bounds were calculated for the full Hamiltonian. Energy expectation values were evaluated by Monte Carlo sampling in the 11ght nuclei, and with the Fermi-hypernettedchain techniques in nuclear matter. The nuclear matter calculations are the first to treat TNI without an "effective" two-body potential approximation.

The realistic TNI bring theory closer to experiment by giving extra b.ading to the light nuclei and reducing the saturation density of nuclear matter. For example, one model with $V_{2 \pi 3 N}$ and $V_{3 N R}$ changes the two-nucleon Urbana- $v_{14}$ results from $-7.2 \mathrm{MeV}$ to $-8.1 \mathrm{MeV}$ in $3_{\mathrm{H}}$, and from $-25 \mathrm{MeV}$ to -29 $\mathrm{MeV}$ in ${ }^{4} \mathrm{He}$ (experimental values are $-8.48 \mathrm{MeV}$ and $-28.3 \mathrm{MeV}$, respectively), while the saturation energy in matter is changed from $-20 \mathrm{MeV} Q p=0.38 \mathrm{fm}^{-3}$ to $-14 \mathrm{MeV}$ a $\rho=0.21 \mathrm{fm}^{-3}$ (empirical value is $-16 \mathrm{MeV} @ \rho=0.16 \mathrm{fm}^{-3}$ ). The Coulomb energy of ${ }^{3} \mathrm{He}$ and the rms charge radil of the light nuclei are also well described. However the charge form factors are not close to experiment, probably due to meson-exchange currents. A paper covering this work has been submitted to Nuclear Physics $A$.

We are currently extending the light nuclei calculations to the excited states of ${ }^{4} \mathrm{He}$, which are seen as resonances in $\mathrm{p}-{ }^{3} \mathrm{H}$ and $\mathrm{n}-{ }^{3} \mathrm{He}$ scattering. We also plan to study meson-exchange-current effects to see if a better description of the form factors can be achieved. The TNI will continue to be an integral part of these studies.

\footnotetext{
*University of Illinols, Urbana, Illinois.
} 
c. Isobar Degrees of Freedom and Three-Body Forces in Nuclear and Neutron Matter

R. B. Wiringa

The effect of three-body forces in nuclear and neutron matter is being studied with both phenomenological three-nucleon potentials and interaction models that explicitly include lsobar degrees of freedom. Phenomenological three-nucleon interaction (TNI) models are of ten based on isobar intermediate-state descriptions, as is the intermediate-range attraction of some NN potentials such as Urbana-v 14 and varlous Bonn models. Alternatively one can construct two-nucleon potentials with both nucleon and Isobar degrees of freedom, which automatically lead to three-body force terms when three-body clusters are considered. Much can be learned about the threebody force by studying these two approaches in parallel.

Nuclear matter calculations with explicit isobars have been developed that treat the isobar on the same level as the nucleon. Correlation operators that induce $N N \rightarrow \mathrm{N} \Delta$ and $N N^{*} \rightarrow \Delta \Delta$ transitions are added to the regular $N N$ correlation operators to produce a sophisticated variational wave function. Fermi-hypernetted-chain techniques used in evaluating normal NN potentlal models are generalized to treat these new operators. Studies with published Nu interaction models show that the higher the fraction of intermediate-range attraction in the two-nucleon system that is provided by coupling to isobar states, the lower the binding energy and saturation density of nuclear matter. Also, $N N^{*} \rightarrow N \Delta$ and $N N^{*+\Delta \Delta}$ transitions seem to be equally important in producing attraction and $\Delta$-state percentage, and there is considerable sensitivity to the djagonal part of the $N \Delta+N \Delta$ and $\Delta \Delta+\rightarrow \Delta \Delta$ interaction that is not apparent in the low-energy two-nucleon phase shift data. There are deficiencies in the published $N \Delta$ interaction models, however, so work is in progress to construct better potentials (see below). To facilitate comparison between the explicit 1sobar model and the phenomenological TNI calculations discussed above, these potentlals will be constructed with a $v_{14}$-type NN part. The otructure and behavior of the explicit isobar model argues strongly for the inclusion of repulsive $V_{3 N R}$ terms in the phenomenological TNI. The calculations with phenomenological TNI in light nuclel and nuclear matter have been repeated with a recently constructed Argonne- $v_{14}$ potential, to test the sengitivity of the results to the choice of two-body force. The Argonne- $v_{14}$ has a $6.1 \%$ deuteron $D$ state and stronger two-body 
tensor correlations than Urbana- $v_{14}$ with 1 ts $5.2 \%$ D state. This in turn generates more attraction for the same TNI model. With some adjustment of the TNI strengths, the Argonne- $v_{14}$ model gives slightly better binding energles and charge form factors for the light nuclei, but nuclear matter results are not Improved. A paper on this work is in preparation. Neutron matter properties are also being calculated to see the effects of TNI on neutron star structure. At twice empirical nuclear matter density, repulsive TNI contributions begin to dominate the total neutron matter energy, and they grow much more rapidly with density than two-nucleon potential contributions. This Implies a stiffer equation of state, and a larger maximum mass for neutron stars. It will also have implications for models of how supernova explosions proceed.

\section{d. Nucleon-Nucleon Potentials with Isobars}

R. B. Wiringa, R. A. Smith, * and T. Afnswurth ${ }^{\dagger}$

NN potential models that include $N_{N+}+\mathrm{N} \Delta$ and $N N++\Delta \Delta$ transitions are being constructed. The intermediate-range attraction observed in $\mathrm{NN}$ scattering can be attributed largely to two-pion-exchange processes with possible isobars in the intermediate state. Part of these processes behaves like a twice-1terated one-pion-exchange potentlal with $\pi N \Delta$ coupling. The description of many physical processes, where 1sobars are believed to play an important role, will benefit greatly from reliable N transition models with good fits to the scattering data.

In present work, the 1sobar is treated as a stable particle. A onepion-exchange potential, containing $\pi N N, \pi N \Delta$, and $\pi \Delta \Delta$ couplings is supplemented by phenomenological intermedlate and short-range terms. The $N N \rightarrow N N$ part is taken in a $v_{14}$ form, $i . e .$, fourteen operator components, and with the transitions added, a total of 26 operators appear in the full potential. The coupled-channel Schrödinger equation is solved in an 1terative manner for the deuteron and all S, $, D, F$, and some $G$ and $H$ waves in the $N N$ channel up to $400 \mathrm{MeV}$. This Includes up to twelve coupled channels in the

\footnotetext{
*Texas A \& M University, College Station, Texas.

† State UnAvers1ty of New York, Stony Brook, New York.
} 
${ }^{3} \mathrm{~F}_{4}-{ }^{3} \mathrm{H}_{4}$ component. The phenomenological part of the potential is adjusted to obtain good fits. The fitting procedure is now in progress.

As an exercise in learning how to efficlently search in a large parameter space for the best fit, the code has been used to construct a conventional NN potential, Argonne- $v_{14}$. This potential was built with the same structure as the Urbana- $v_{14}$ model, for which rellable variational manybody calculations have been developed. However, Argonne-v 14 was fit to more recent phase-shift data and came out with a significantly higher deuteron Dstate peri-entage. It is currently being used in a study of phenomenological three-body forces (see above).

In future work it would be useful to fit the scattering data at higher energies, including inelasticities. In particular the possible existence of resonances in the $S-$ and $P$-wave $M$ system, which would show up in the ${ }^{l_{D_{2}}}$ and $3_{F_{3}}$ NN channels. could place significant constraints on the nature of the diagonal $N \Delta+\rightarrow N \Delta$ interaction. To make such fits, relativistic effects and the Instability of the $\Delta$ will have to be taken into account. We hope to tackle this problem in the next year.

e. Phenomenological Hamiltonian for Pions, Nucleons and $\Delta$ Isobars T.-S. H. Lee

We have been constructing a many-body Hamiltonian for a nuclear system consisting of pions, nucleons, and $\Delta$ isobars. Such a model is needed to study both the intermediate energy pion-nucleus interaction and the mesonic degrees of freedom in nuclei. The basic mechanisms of the model are parameterized in terms of: (a) a $\pi N^{*} \rightarrow \Delta$ vertex interaction $h$ in the resonant $(3,3)$ channel, (b) a two-body interaction $v_{\pi N}$ in other $\pi N$ channels, and (c) a two-body interaction $V_{0}$ between all two baryon channels $N N, N \Delta$ and $\Delta \Delta$. The parameters are determined by fitting the $\pi \mathrm{N}$ phase shifts up to $300 \mathrm{MeV}$ and $\mathrm{NN}$ phase shifts up to $1 \mathrm{GeV}$. Neglecting $\Delta \Delta$ channel and using separable kernel to parameterize all two-body interactions in each partial wave, a many-body Hamiltonian of this type was obtained by Betz and Lee in 1981 and has since been used in the study of pion absorption by nuclet. We are working on improvements of the model. The conventional one-boson-exchange (OBE) mechanism is used to parameterize the two-baryon interaction $v_{0}$. As a first step, we use the Bonn OBEP model for $V_{0}$ and keep only the imaginary parts of 
the contributions due to one-pion exchange between $M$ channels and the $N N$ interaction in the $\pi N N$ channel. The $\Delta$ is dressed exactly by $\pi N+\Delta$ interaction. It is found that: (a) below $300 \mathrm{MeV}$, the NN scattering phase shifts in $\ell>0$ partial wave are not changed significantly when $\Delta$ is treated as an unstable particle, (b) the $\mathbb{N N}$ interactions in $\pi N N$ channel play an important role in determining the NN Inelasticity, and (c) the relativistic kinematics in the NN channel must be treated properly in all partial waves at E>400 MeV. Our present efforts focus on developing methods for an exact treatment of all of the singularitles in the interacting $\pi N N$ channels. The numerical method being employed is Fuda's method which does not rely on contour rotation in the complex plane and is therefore sultable for our calculation using the OBE model. A computer program is being developed for a construction of a many-body Hamiltonian based on the OBE mechanism.

\section{f. Relativistic Quantum Mechanics of Particles with Direct Interactions}

F. Coester and W. N. Polyzou*

Nuclear many-body systems are predominantly nonrelativistic. Nevertheless relativistic effects (i.e., effects due to the difference between Galilean Invariance and Lorentz invariance) are not negligible. Furthermore an explicit treatment of mesonic and isobar degrees of freedom cannot be Galilean invariant. If the sum of the particle masses is not conserved, a theory invariant under boosts must be relativistic. There are two minimal requirements for relativistic quantum mechanics: (1) There must be a unitary representation of the Lorentz transformations, space and time translations. (2) The theory must be at least macroscopically local. (Two systems localized in relatively spacelike reglons must be dynamically independent.) Starting with two-body interactions we have satisfied both requirements by an explicit recursive construction of a Hamiltonian and the assoclated Lorentz boosts for an arbitrary fixed number of particles. Many-body Hamiltonians so constructed share many familiar features with nonrelativistic many-body Hamiltonians, and nonrelativistic approximations are straightforward. Preliminary results have been reported at the Barcelona Workshop "Relativistic Action-at-a-Distance. Classical and Quantum Aspects," June 1981. We have shown that this construction can be generalized to allow for particle creation. In that case

\footnotetext{
*Massachusetts Institute of Technology, Cambridge, Massachusetts.
} 
the complete system will have ilfinitely many degrees of freedom but not the divergences of local field theorles. A paper on this work will be submitted to Phys. Rev.

g. Strong Coupling Approximations to Chiral Bag Models

John Parmentola

Over the past year, we have made significant progress in developing and applying strong coupling approximations to chiral bag models, which describe the interaction of the local pion fleld to quarks inside a bag. These models are particularly Important for a more fundamental understanding of the pion-nucleon interaction, the nucleon-nucleon force, the pionic corrections to the static properties of the baryons such as the mass spectrum, and the connection between the quark model description of the delta and the delta reso-iance observed in pion-nucleon scattering. In these models, the strength of the pion-nucleon coupling increases as the nucleon bag radius decreases. Phenomenological studies of the nucleon-nucleon force indicate that the bag radius is of the order of the proton Compton wavelength; therefore strong coupling is important. For strong coupling, we have developed and applied an approximation method to an SU(2) symmetric static bag source meson field theory in which the nucleon only has isospin degrees of freedom. We have calculated the spectrum of the Hamiltonian and the corresponding wavefunctions. We have shown that the strong coupling perturbation expansion converges to the lowest order results when the bag radius goes to zero and the coupling constant goes to infinity.

For practical computations, we have demonstrated that there exists a range of physically relevant values of the bag radius and coupling constant for thich the perturbations are small. Furthermore, for sufficlently large coupling we have shown that the 1sobar excltation of the nucleon becomes stable. We have generallzed the model by adding a static 1sobar source coupled to the meson fleld. We have shown that for fixed bag radius, the strong coupling approximation is realized for smaller values of the coupling constant. In addition, we have shown that there exists a thirty percent admixture of the 1sobar in the dressed nucleon wavefunction.

Currently, we are applying the method of calculation to realistic models. Once this has been completed, we will solve the plon-nucleon scattering problem for strong coupling which will establish the connection 
between the quark model delta and the delta resonance observed in pion-nucleon scattering. Furthermore, our method of calculation opens up the possibility of understanding other pion-nucleon resonances such as the Roper resonance. Finally, we expect to calculate pionic corrections to the static properties of the baryons and the nucleon-nucleon force. 


\section{INTERMEDIATE ENERGY PHYSICS}

T.-S. H. Lee, J. Parmentola, and others

In 1981 a many-body Hamfltonian was constructed by Betz and Lee for studying the pion-nucleus interaction by including the $\Delta$ degree of freedom. This Hamlj.tonian has now been applied to various specific problems of scattering and absorption of pions with encouraging results. Our DWIA code for pion nucleus inelastic scattering is being used to interpret experiments of the Argonne group and other users of LAMPF. Work has also been done on elastic and inelastic scattering of protons with energy in the 0.5 to $1 \mathrm{GeV}$ region.

\section{a. A Microscopic Study of $\Delta$-Nucleus Potentials}

T.-S. H. Lee and K. Ohta*

In the one-hole line approximation, the self-energy of $\Delta$ propagation in nuclear matter is calculated from the many-body Hamiltonian for $\pi, N$ and $\Delta$ constructed by BeLz and Lee. In the local density approximation, the calculated $\Delta$ self-energy corresponds to the strength of the $\Delta$-nucleus potential which is determinet phenomenologically in the $\Delta$-hole doorway model of pion-nucleus scattering. It is found that our calculated strength of the central potential is on the average about $80 \%$ of the empirical one. However, the calculated spin-orbit potential is much smaller than that of the $\Delta$-hole mode1. We also find that pion absorption through the $N \Delta s$ wave only accounts for about half of the total absorption. The sum of the other partial waves is found to be equally important. A paper describing our study has been accepted for publication in Physical Review $C$.

\footnotetext{
${ }^{\star}$ Massachusetts Institute of Technology, Cambridge, Massachusetts.
}

b. A Study of the Inclusive Reaction ${ }^{4} \mathrm{He}(\pi, \mathrm{p})$

T. S. H. Lee and A. K. Kerman*

The inclusive reaction ${ }^{4} \mathrm{He}(\pi, p)$ is studied by assuming that the basic absorption mechanism is $\pi N N+N \Delta+N N$ as described by the model of Betz and Lee. Our main effort here is to examine the effect of ground-state correlation on the proton spectra. The calculations have been done by assuming that the inftial pion and final proton can be treated as plane waves, while the 
$\pi N N \rightarrow N \Delta \rightarrow N N$ amplitude is calculated using the entire Betz-Lee model. It is found that the NN correlation in the ground state of ${ }^{4} \mathrm{He}$ can broaden the proton spectra and increase the differential cross section by as much as a factor of 2. The calculated results for $\pi^{+}$absorption are in qualitative agreement with the data but we are not able to obtain reasonable results for $\pi^{-}$absorption. A more careful study of initial and final state interactions is clearly needed for a detalled understanding of pion absorption by nuclel.

c. Scattering and Absorption of Plon by the $A=3$ Nuclei T.-S. H. Lee and K. Ohta*

Within the many-body Hamiltonian model of Betz and Lee, we are Investigating both the scattering and absorption of plons by ${ }^{3} \mathrm{He}$ and ${ }^{3} \mathrm{H}$. Our objective is to examine the relative Importance between the two-nucleon and the three-nucleon absorption mechanisms, and their influence on the scattering channel. Keeping only the intermediate states involving at most one pion and neglecting three nucleon interactions in the $\pi$ NNN channel, we express both the scattering and absorption amplitudes in terms of a three-body amplitude defined in the coupled NNN@NNS subspace. The resulting three-body equation can be solved by using a numerical method due to Fuda. We expect to obtain results in 1982 and compare them with the forthcoming data of coincidence measurements by the ANL group.

\footnotetext{
*Massachusetts Institute of Technology, Cambridge, Massachusetts.

d. Intermediate Energy Electron Scattering T.-S. H. Lee and K. Ohta*

The dominant mechanism in $\left(e, e^{\prime}\right)$ on nuclel when the energy transferred to the nucleus is about $300 \mathrm{MeV}$ involves excitation of a nucleon to the $\Delta$ state. The kinematic conditions of $\Delta$ creation by the virtual photon, $\gamma N^{N \rightarrow \Delta}$, are quite different from those in the pion-nucleus interaction. Since the virtual $\gamma$ interacts weakly with the nuclear medium, the $\Delta$ can be produced throughout the nuclear volume, whereas the pion produces $\Delta$ 's mainly on the nuclear surface. Furthermore if the incident electron energy is several GeV,
}

\footnotetext{
*Massachusetts Institute of Technology, Cambridge, Massachusetts.
} 
one can produce very fast off-shell $\Delta$ 's with momenta of many GeV/c. The $\Delta$ produced in the $\pi$-nucleus interaction is mainly on-shell with momentum about $0.3 \mathrm{GeV} / \mathrm{c}$. Therefore $\left(e, e^{\prime}\right)$ study can examine the dependence of $\Delta$-nucleus dynamics on the nuclear density and on the $\Delta$ momentum. We are studying (e,e') on $A=3$ nuclei by adding the $\gamma \mathbb{N}+\Delta$ vertex interaction to the Betz-Lee model. One can then express the $\left(e, e^{\prime}\right)$ amplitude in terms of the $\triangle N N+N N N$ three-body amplitudes of the model, and predict results expected from experiments with future electron accelerators of several $\mathrm{GeV}$ and high duty factor.

\section{e. Spin and Isospin Effects in Elostic $\mathrm{p}^{-4} \mathrm{He}$ Scattering Near $1 \mathrm{GeV}$} J. A. Parmentola and H. Feshbach*

The coupled channel method for multiple scattering is employed to describe $\mathrm{p}^{-4}$ He elastic scattering data for a proton laboratory energy of 1.03 GeV. Particular attention is paid to the double spin flip, double isospin flip, double spin-isospin flip and to the effects of lsobar excitation of the incident proton. It is found that the presently available data are consistent with either the excitation and deexcttation of the proton to an 1sobar by the ${ }^{4}$ He nucleus or the presence of relatively large double spin flip nucleonnucleon amplitudes. The physical difference between these two hypotheses is discussed. (Accepted for publication In Annals of Physics.)

\footnotetext{
*Massachusetts Institute of Technology, Cambridge, Massachusetts.
}

\section{f. Elastic and Inclusive Proton-Nucleus Scattering Above $500 \mathrm{MeV}$} J. A. Parmentola and H. Feshbach*

Our model is based on the KMT formalism and includes the essential physics which is important at these energies, namely the distortion of the elastic wavefunction and its coupling to a set of inelastic channels, the final state interactions of the proton and the residual nucleus and the final state interactions of the knocked out nucleons with the residual nucleus. The model is obtained by truncating the KMT series for the optical potential to second order in the free $\mathrm{N}-\mathrm{N}$ t-matrix and assuming the closure approximation. Upon expanding the second order optical potential in an

\footnotetext{
*Massachusetts Institute of Technology, Cambridge, Massachusetts.
} 
appropriate basis, and substituting the first and second order elastic optical potentials in the Schrödinger equation, we obtain a set of coupled channel equations which have the essential physics described above. We have shown that this basis expansion converges very rapidly and therefore the nurber of inelastic channels are few in number. However, the inelastic channels do not correspond to physical channels but provide an economical representation of the many channels which are important at these energies. Since the model is based on a coupled channel formalism, the elastic and inelastic scattering are determined in a consistent manner. The solution of these equations is used to determine inclusive scattering in the closure approximation. 
E. MICROSCOPIC CALCULATIONS OF HIGH-ENERGY COLLISIONS OF HEAVY IONS

A. R. Bodmer

The study of heavy-ion collisions at energies above about 100 $\mathrm{MeV} /$ nucleon seems the only means of learning about the properties of nuclear matter at high densities and temperatures at which new states of nuclear matter may exist. Because the mean free path is relatively large, nonequilibrium phenomena are expected to be important and an adequate description of such collisions must in general be a microscopic one. We initiated and are continuing classical-equations-of-motion (CEOM) calculations of high-energy heavy-ion (HE-HI) collisions. The CEOM method is a completely microscopic but classical approach whose essence is the calculation of all nucleon trajectorles using a two-body potentlal between all palrs of nucleons. The unique feature of this approach is that it includes finiterange interaction effects (in particular potentlal energy effects) and hence does not assume that nuclear matter is a dilute gas as do cascade calculations. On the other hand, in contrast to hydrodynamics, the CEOM approach is a microscopic one and does not assume local thermodynamic equilibrium. Thus with the CEOM approach we were able to investigate different 2-body potentials which give identically the same 2-body scattering, but which for nucleus-nucleus collisions give very different potential energies - and therefore equations of state -- since the densities are such that three or more nucleons interact simultaneously.

a. Classical-Equations-of-Motion Calculation of Multiplicities for HighEnergy Collisions of ${ }^{20} \mathrm{Ne}+{ }^{20} \mathrm{Ne}$

A. R. Bodmer and C. N. Panos

A paper with the above title has appeared in Nuclear Physics A356, 517-522 (1981). This paper obtains nucleon multiplicity distributions as a function of impact parameter and discusses their usefulness as an indicator of impact parameter.

b. Interaction and Collective Effects in Classical-Equations-of-Motion

A. R. Bodmer

The evolution of central equal mass nucleus-nucleus collisions as obtained with the CEOM approach is discussed, with special reference to interaction and collective effects. Inttially single scattering dominates followed by rapid development of multiple scattering leading to approximate thermalization near maximum compression. Up to this time conditions are mostly far from thermalized and a hydrodynemic description is inappropriate. Finally there is an expansion for which "collisions," interpreted as due to the short-range repulsion in the 2-body force, are relatively ineffective and 
when interaction effects are dominated by streaming in an average potential mostly due to the long range attractive part of the 2-body potentlal. Whereas for ${ }^{20} \mathrm{Ne}+{ }^{20} \mathrm{Ne}$ there is forward peaking in the finai nucleon distribution, for ${ }^{40} \mathrm{Ca}+{ }^{40} \mathrm{Ca}$ there occurs transverse peaking which is an indication of collective effects. This onset of collective effects for larger nuclei is interpreted as the result of an interplay between transparency effects and interaction effects due to the repulsive core of the 2-body potential. These results will appear in the Proceedings of the 5th High Energy Heavy Ion Study (LBL, Berkeley).

\section{c. Improvements and Extensions of the CEOM Calculations}

A. R. Bodmer

The CEOM calculations are being developed in order to extend their scope and flexibility. Momentum dependent 2-body potentials together with other procedures are being developed in order to obtain more satisfactory binding and 2-nucleon scattering properties. This will allow study of a larger range of projectile and target nuclei, and in particular make it possible to obtain more realistic estimates of the compressions obtainable in $\mathrm{HE}-\mathrm{HI}$ collisions. We belleve none of the existing theoretical estimates for the compressions are very realistic.

Another extension we are considering is to allow for Pauli blocking effects in the one-body phase space. This requires incorporation of a probabilistic element in the scattering, either through the scattering by the repulsive core or by considering the two-body scattering as a whole. Such an extension would make the approach applicable to higher densities and especially to lower energies, namely in the range of about $50-200 \mathrm{MeV} /$ nucleon where there is currently no sultable microscopic description.

\section{d. Theory of High-Energy Heavy-Ion Collisions}

A. R. Bodmer

An earlier analysis of the different basic approaches to high-energy heavy-ion collisions (in particular, hydrodynamics, cascade calculations, CEOM calculations) clarified the complementary nature of the approaches and their respective merits. An unpublished review of this analysis is being revised and brought up to date. 


\section{F. OTHER THEORETICAL PHYSICS}

\section{a. Aharonov-Bohm Effect}

Murray Peshkin

According to conventional quantum mechanics, the behavior of a charged particle can be influenced by magnetic fields in a region from which the particle is rigorously excluded. This phenomenon, called the AharonovBohm effect, has attracted considerable attention because it challenges our underscanding of locality in electromagnetism. It also plays a role in the understanding of flux quantization in superconductors and in magnetic monopole theories.

I recently pointed out [Physics Reports 80,375 (1982)] that the $A B$ effect is in one sense due to the penetration of the magnetic field region by the electric field whose source is the electron. This does not change the classical motion of the electron, but the crossed fields contain angular momentum, and in quantum mechanics that has measurable consequences because angular momentum is quantized.

In the course of this work, a question came up as to whether electrostatic shielding of the magnetic fleld region could prevent the $A B$ effect from being observed in certain experiments. It has now been proved that this cannot be the case for all experiments so the $A B$ effect is observable in principle, but a question remains about some experiments, possibly including all of those which have been performed to date. I plan to address this question in the near future.

b. Coupled Channel Formalism for Rearrangement Reactions M. Kawai, M. Ichimura, ${ }^{*}$ and N. Austern ${ }^{\dagger}$

The relation between the method of coupled channels for rearrangement reactions (CRC) and the bound state approximation to the channel coupling array formalism (BSCCA), advocated in recent years, is investigated in detail

\footnotetext{
*Unfversity of Tokyo, Tokyo, Japan.

tUniversity of Pittsburgh, Pittsburgh, Pennsylvania.
} 
for a siuple 3-body system expressed in terms of truncated component wavefunctions of Faddeev type. The system is described by coupled differentlal or integral equations that are truncated into a model space of strongly-coupled channels. It is shown that CRC can be derived from the truncated coupled equations, elther in differential or integral form, provided care is taken to use the entire model space. The corresponding BSCCA in this model space can be obtained from a restrictive condition on the integral form of the coupled equations, while it cannot be obtained consistently from the differential form of the coupled equations. The boundary conditions for the component functions are discussed in deta11.1

\footnotetext{
1M. Kawai, M. Ichimura, and N. Austern, Z. Phys. A303, 215 (1981).

c. Applicability of the Potentlal Model of Colored Quarks for Nucleon Interactions

Harry J. Lipkin

The additive potential model of colored quarks successfully accounts for the masses, decay rates and other properties of single mesons and baryons. However, attempts to calculate the nucleon-nucleon force with this model lead to long-range inverse-power Van der Waals potentials between nucleons which are In substantial contradiction with experimental data. A discussion of this issue has been published. 1
}

10. W. Greenberg and H. J. Lipkin, Nucl. Phys. A370, 349 (1981).

d. Model Independent Analysis of Experimental Baryon Magnet1c Moments Harry J. Lipkin

The naive constituent quark model is quite successful in describing the hadron mass spectrum and accounting for the observed magnetic moments of the nucleon and the lambda. However, strong disagreement is found for the function of hyperon magnetic moments $R\left(p, \Sigma^{+}, \Xi\right)=3\left(\mu_{p}-\mu_{\Sigma}+\right) /\left(\mu_{\Sigma^{-}}-\mu_{\Xi^{\circ}}\right)$. The experimental value is $R=2.7 \pm 0.8$ which is an order of magnitude larger than the broken SU(6) prediction of $R=0.34$. A model independent analysis has been performed including SU(6) symmetry breaking, configuration mixing, relativistic corrections and quark-diquark correlations. The conclusion is that the observed value of $R$ implies quenching of contributions of non-strange 
quarks in strange-beryon magnetic moments relative to contributions in nucleon magnetic moments. This result is published In Phys. Rev. D 24, 1437 (1981). 


\section{THE SUPERCONDUCTING LINAC}

R. Benaroya, L. M. Bollinger, R. C. Pardo, K. W. Shepard, G. P. Zinkann, J. Aron, * B. E. Clifft, ${ }^{*}$ K. W. Johnson, * P. Markovich, ${ }^{*}$ and J. M. Nixon ${ }^{*}$

\section{INTRODUCTION}

The antivities concerned with advancing the technology of the superconducting heavy-ion linac now have three major components. One is the specific task of completing the prototype superconducting linac that functions as an energy booster for heavy ions from the tandem electrostatic accelerator. The gecond part consists of continuing investigations of various aspects of superconducting $\mathrm{rf}$ technology, some of which are of fairly general interest for accelerator technology. The third, a new undertaking, is the line-item project to extend the present tandem-1inac accelerator into a substantially larger system called ATLAS.

All parts of the superconducting linac program are jointly supported by the Chemistry and Physics Divisions.

\footnotetext{
${ }^{\star}$ Chemistry Division, ANL.
} 


\section{A. PROTOTYPE HEAVY-ION SUPERCONDUCTING LINAC}

Th1s project, started in mid-1975, is concerned with the design, construction, installation, and testing of a small superconducting linear accelerator (1inac) to serve as an energy booster for heavy-ion beams from the FN tandem accelerator. The princlpal objectives of the project are to develop a new accelerator technology and to build the prototype for a heavy-ion energy booster that can be used to upgrade the performance of any tandem accelerator. The overall design is highly modular in character in order to provide maximum flexibility for future modifications and/or 1mprovements. Twenty-two resonators of the planned 24-resonator system were in place in March 1982, and the final two resonators will be completed this year.

By the end of the present reporting period, the booster was being used routinely to provide beams for nuclear-physics research. This operational experfence 18 summarized in Sec. VII of this document.

\section{STATIS OF THE BOOSTER}

The booster linac is now complete in all essential respects, except for installation of the last two resonators. The maln features of the design were outlined in last year's Annual Review. Considerable effort is still being devoted to perfecting various subsystems of the accelerator, as outlined in Sec. VII, and this effort is resulting in a machine of excellent rellabllity. By the end of 1982 the developmental effort still being devoted to the booster will have shifted almost entirely to the ATLAS project.

\section{NEAR-TERM PLANS}

The most obvious task for 1982 is to complete and Install the last two resonators of the 11nac. This is expected to occur in October, at which time the performance of the tandem-linac system will be equivalent to that of a 25-MV tandem with two strippers for lons in the lower half of the periodic table.

Another important remaining task is the development and installation of an improved slow-tuner system. Because of component fallure, the pneumatic tuning system used until now has been a primary source of operational inefficiency.

A continuing effort will be devoted to refining many other subsystems, Including especlally the beam-energy measurement system, the computer programs uged to control the linac, and the refrigeration system. 


\section{B. INVESTIGATIONS OF SUPERCONDUCTING LINAC TECHNOLOGY}

This program, carried out fointly by the Chemistry and Physics Divisions, is concerned with investigations of the general aspects of applications of superconducting technology to the acceleration of heavy ions. Most of the recent activities have been related to the development of accelerating structures of the split-ring type made of nioblum. Investigations of this type will continue, but the program is broadening steadily to Include developmental work on other aspects of accelerator technology, Including (1) the development of superconducting magnets for use in bending, controlling, and analyzing heavy-ion beams and (2) the advancement of time-of-flight technology.

The cholce of all recent disvelopmental work has been gulded by the urgent developmental needs of the heavy-ion booster and the ATLAS project. Nevertheless, most of the Investigations undertaken are of general interest to superconducting-linac technology.

\section{RECENT ACCOMPLISHMENTS}

\section{a. Superconducting Resonators}

K. W. Shepard

Work is proceeding on a broad front on the development of superconducting resonators for heavy-1on acceleration. This includes (1) the development of a new class of resonators for use in ATLAS, (2) the continuing study of the performance of on-line resonators, and (3) the development of a new type of slow tuner.

\section{(1) The ATLAS Resonator}

For ATLAS it is desirable to have a resonator that is maximized for an ion velocity that is substantially higher than the present $B=0.105$ unit. After a thorough design study, it was conciuded chat the best cholce for our needs would be a split-ring resonator operating at $147.5 \mathrm{MHz}, 3 / 2$ times the rf frequency of the present resonators. This choice maxir,1zes the acceleration of ions with $\beta=0.16$. By optimizing the shape of all parts of the drift tube assembly, it is expected that the accelerating field will be Increased.

Because of new requirements on the shape of the drift-tube assembly, fabrication of the ATLAS resonator is a challenge. Work on the prototype unit proceeded throughout the past year and was completed in late January 1982. 
Testing of the new prototype has started. For low-fleld operation, the unit has an excellent $Q$ of about $1.5 \times 10^{9}$, but for reasons that are not yet understood the accelerating field 1 imits at the low value of about 1.9 NV/ni. Since this performance is not acceptable, the fabrication of a second unit has been started.

\section{(ii) On-Line Performance of Resonators}

Because of the pressure of other work, during the past year almost no effort was devoted to increasing the accelerating fields of the resonators in the on-1ine cryostats. Nevertheless, we mate the significant observation that the performance of the resonators did not change to a measurable extent during a year of routine steady use. Of the 21 resonators in operation, those that started the year with exceptictally good characteristics maintained those good characteristics, whereas those that had been previously degraded in various ways did not improve. In connection with this result, it is probably significant that there were no important vacuum accidents during 1981 .

\section{(111) Slow Tuner Controller}

Last year we reported on the development of a new form of pressure controller for the resonator slow tuner, a pneumatic device used to vary the resonator frequency by deflecting an end plate. The new pressure controller consists of a heater in a closed chamber operating at liquid helium temperature. Because of the low temperature, a change in temperature causes a relatively large change in pressure.

During the past year, the new pressure controller was put into service on 15 resonators, thits providing a thorough operational test. The device was found to be excellent in terms of stability and reliability, but it does not have as much pressure range as 18 needed for some modes of operation of the resonators. Therefore, other forms of pressure controller are to be examined.

\section{b. Time-of-F1ight Technology}

R. C. Pardo and R. N. Lewis*

From the inception of the superconducting-1inac project it has been our alm to develop new approaches to using time-of-flight techniques for

\footnotetext{
*Eleccronics Division, ANL.
} 
accelerator control and diagnostics. For a pulsed machine with the range of energy and magnetic rigidity provided by our linac, it is belleved that timeof-flight techniques can be better and less expensive than magnetic techniques for some purposes. Hence, we have invested a considerable effort in the development of new time-of-flight techniques, and this effort has now begun to yleld good results.

\section{(1) Linac-Beam Tuning}

Last year we described a new approach to tuning the 11nac phase by using a time-of-flight technique to measure the beam-energy change as a function of resonator phase. The detector for these measurements is a beamexcited resonator. The objective is to develop a technique by which the Inac can be tuned very rapidly and accurately.

Work on the new tuning technique has proceeded throughout the past year. The essential validity of the technique was established in the first trials, but various technical problems have prevented the new system from being routinely useful. The main challenge is to develop electronics that can rellably measure the beam-exclted phase when the beam is weak and flickering.

The greater success achieved with two simflar detectors in the beamenergy measurement system described below shows that there is no inherent reason why a beam-excited resonator should not function well for beam tuning.

\section{(1i) Beam-Energy Measurement}

A beam-energy measuring system that makes use of two beam-exc1ted resonators has been developed and put into service during the past year. The basic 1dea of the system is to measure the phase difference, and hence the bunch-arrival-time difference, of the two resonators placed about 10 meters apart on the beam line. Each beam-excited resonator is the same as the one used for beam tuning, but the assoclated electronics is of an improved design.

The complete energy-measurement system is now in routine operation, although it 18 st1ll being refined. At the present stage of development, it provides a continuous, nondestructive readout of beam energy with an accuracy of a few parts in $10^{3}$.

It 18 expected that the accuracy w11l ultimately be improved by an order of magnitude. 


\section{(11i) Beam-Energy Control}

In addition to measuring the beam energy, the two-resonator system described above in (i1) is designed to control the energy of the linac beam. Th1s will be done by correcting a wrong energy by virying the phase of the rebuncher on the output beam ifne. This approach 18 expected to control the mean energy of the beam to an accuracy of a few parts in $10^{4}$.

Tests on beam-energy control are about to start.

\section{(iv) Beam Bunching}

The study of the technology of beam bunching has continued, with emphasis on rebunching the beam from the 1inac. Although it has been demonstrated that beam impulses as narrow as 50 ps can be formed under favorable geometrical conditions, under the geometry Imposed by our target

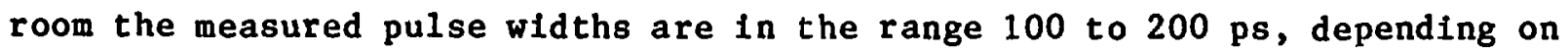
the ion species involved. This range of performance 18 not 11 kely to be 1mproved until the system is changed in at least one of two respects: (1) the rebuncher is moved nearer to the detector and/or (2) the beam quality is improved by moving the second stripper near to the time focus at the linac entrance.

\section{c. Superconducting Analyzing Magnet}

R. C. Pardo and R. P. Smith*

As reported last year, some effort has been devoted to the development of a prototype superconducting magnet. This magnet was orginally planned as a prototype for a pair of magnets to be used as an analyzing system for the tandem beam, but 1 t was soon evident that the same design would be useful for the $40^{\circ}$ bend in ATLAS and as a swltch magnet in the ATLAS experimental area.

Work on the prototype superconducting magnet has progressed very slowly during the past year because of a shortage of manpower and money and the greater urgency of other tasks. However, the work has proceeded gradually, and varlous essentlal tasks have been completed, including design of the cryostat and machining of the yoke and magnetic shield.

Now that ATLAS has been funded, completion of the superconducting magnet has taken on greater urgency, and work has started again on all aspects

\footnotetext{
${ }^{*}$ Accelerator Research Facilities Division, ANL.
} 
of the profect. If successful, as expected, the magnet w111 be used as one of the two superconducting magnets planned for ATLAS. It will also continue to serve as the prototype for the magnets needed for the analyzing system for the tandem beam.

\section{NEAR-TERM PLANS}

Much of the effort during the coming year will continue to be devoted to problems that are closely related to the needs of ATLAS and the refinement of the present 11nac.

The highest priority will be given to the completion of development of the resonatior for ATLAS.

Now, after several years of effort, we finally have the tools required for a systematic study of all aspects of the beam quality achlevable with the superconducting 1inac. These tools include the energy-control system being put into service, a functioning rebuncher, and a functioning magnetic spectrograph. These will be used to study (a) the long-term stability of beam energy, (b) the value of the longitudinal emittance $\triangle E \Delta t$ as a function of ion specles and stripping foll characteristics, (c) the time and energy resolution achievable under various conditions, and (d) the dependence of beam quality on the location of the second stripper.

Work on the development of a good pressure controller for the slow tuner w11l proceed immediaiely.

The development of the superconducting dipole magnet will extend into 1983. By then, the basic fabrication will be completed, and fleld mapping and operational tests will be carried out. This work is viewed as being of great importance to our program since we need at least four large dipoles in the final ATLAS system.

Design studies on the feasibility of a superconducting septum magnet will start in 1983.

Studies of electron loading in superconducting resonators will continue. Th1s work has been seriously hampered until now by the fact that all good resonators have been tied up in beam-line use. However, this problem will disappear in late 1982 . 


\section{THE ATLAS PROJECT}

The Argonne Tandem.'Linac Accelerator System (ATLAS) is a heavy-ion accelerator to be formed by en?arging the booster and by adding a large new target area, as shown in Fig. VI-1. The resulting system, consisting of the existing tandem and a 7-section linac, will have a performance that is approximately equivalent to that of a 50-MV tandem with two strippers.

The ATLAS project was authorized in the FY 1982 budget at a level of $\$ 7.7$ million, and $\$ 4.0$ million was appropriated in FY 1982 . The project started officlally in January 1982 when these funds were released. The remaining $\$ 3.7$ million 1s expected to be appropriated in FY 1983.

ATLAS is aimed squarely at the needs of precision nuclear-structure physics, providing beam energies up to $25 \mathrm{MeV} / \mathrm{A}$, easy energy variability, and beams of exceptionally good quality. The short-pulse character af the beam w11 be emphasized so as to maximize its usefulness for time-of-flight and other timing measurements. An unusual feature of the factlity is the capability of providing beams simultaneously for two independent experiments without a loss of intensity to either.

One of the main technical objectives of the ATLAS project is to add three linac sections that can effectively accelerate the relatively fast projectiles produced by the present 11nac. This requires the development and fabrication of a new accelerating structure that is optimum for lons wish velocity $\beta=0.16$, considerably greater than our present $\beta=0.105$ structure. After an extensive design study, it was concluded that the best structure for our needs is one with $\beta=0.16$ and a frequency that 1 s $3 / 2$ t:imes greater than the $\mathrm{rf}$ frequency $(97 \mathrm{MHz})$ of the $\beta=0.060$ and $\beta=0.105$ structures used in the tooster. The two frequencles can be mixed only becaluse the highest desired frequency of the beam pulse $1897 / 2 \mathrm{MHz}$, a subharmonic of both $97 \mathrm{MHz}$ and $145.5 \mathrm{MHz}$. The planned cunfiguration of resonators in ATLAS is shown in Fig. VT-2.

Because the accelerator addition involved in ATLAS is closely simflar to the existing prototype linac, it was possible to start the work on major components as soon as funds were released. The fabrication of one cryostat and 1 t $B$ resonators started immediately, with the objective of starting assembly in early 1983. 


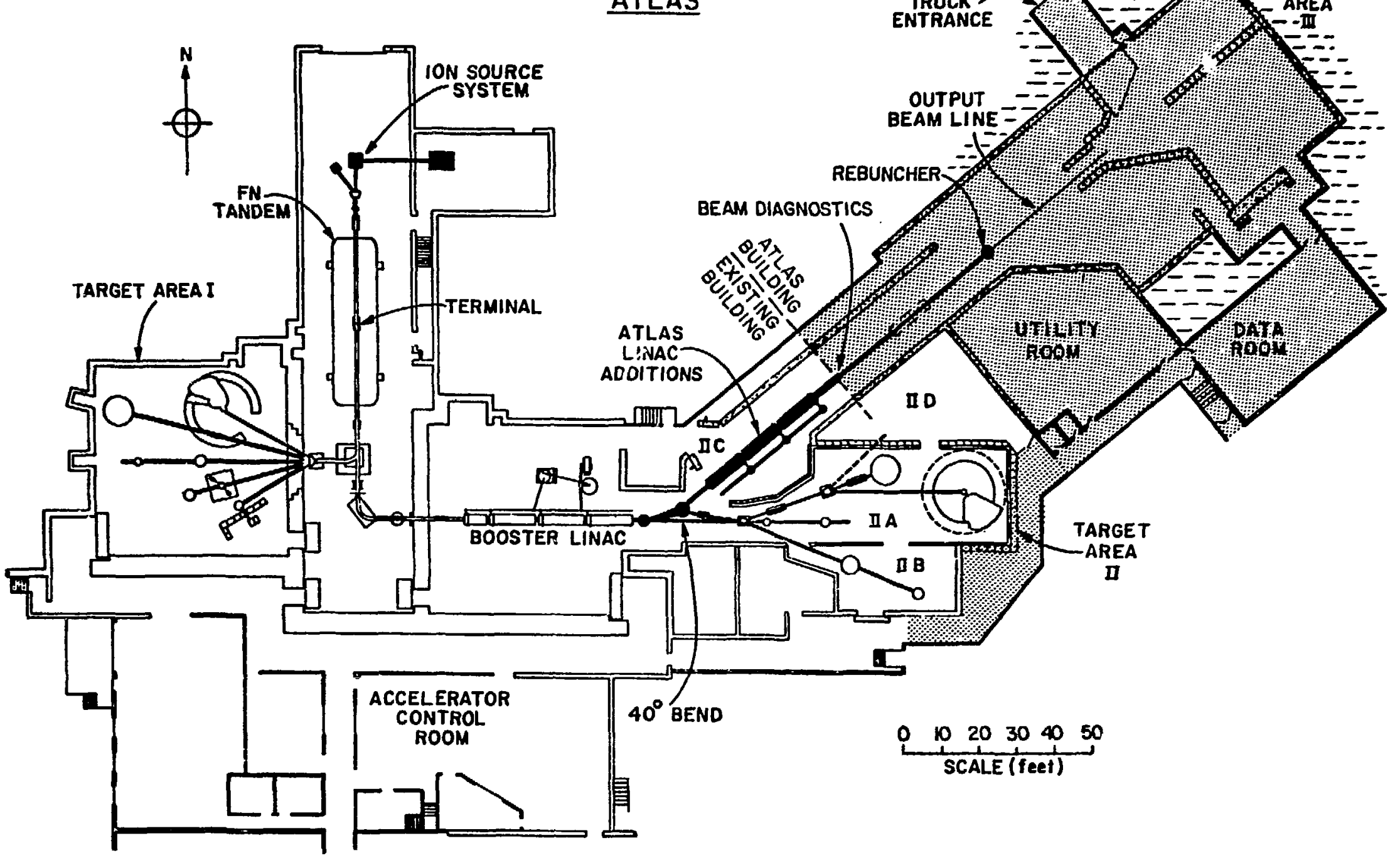

Fig. VI-1. Layout of the ATLAS facility, where the building areas shown in grey (dotted) are to be added by the ATLAS project. The new accelerator components to be added are shown fully darkened. 


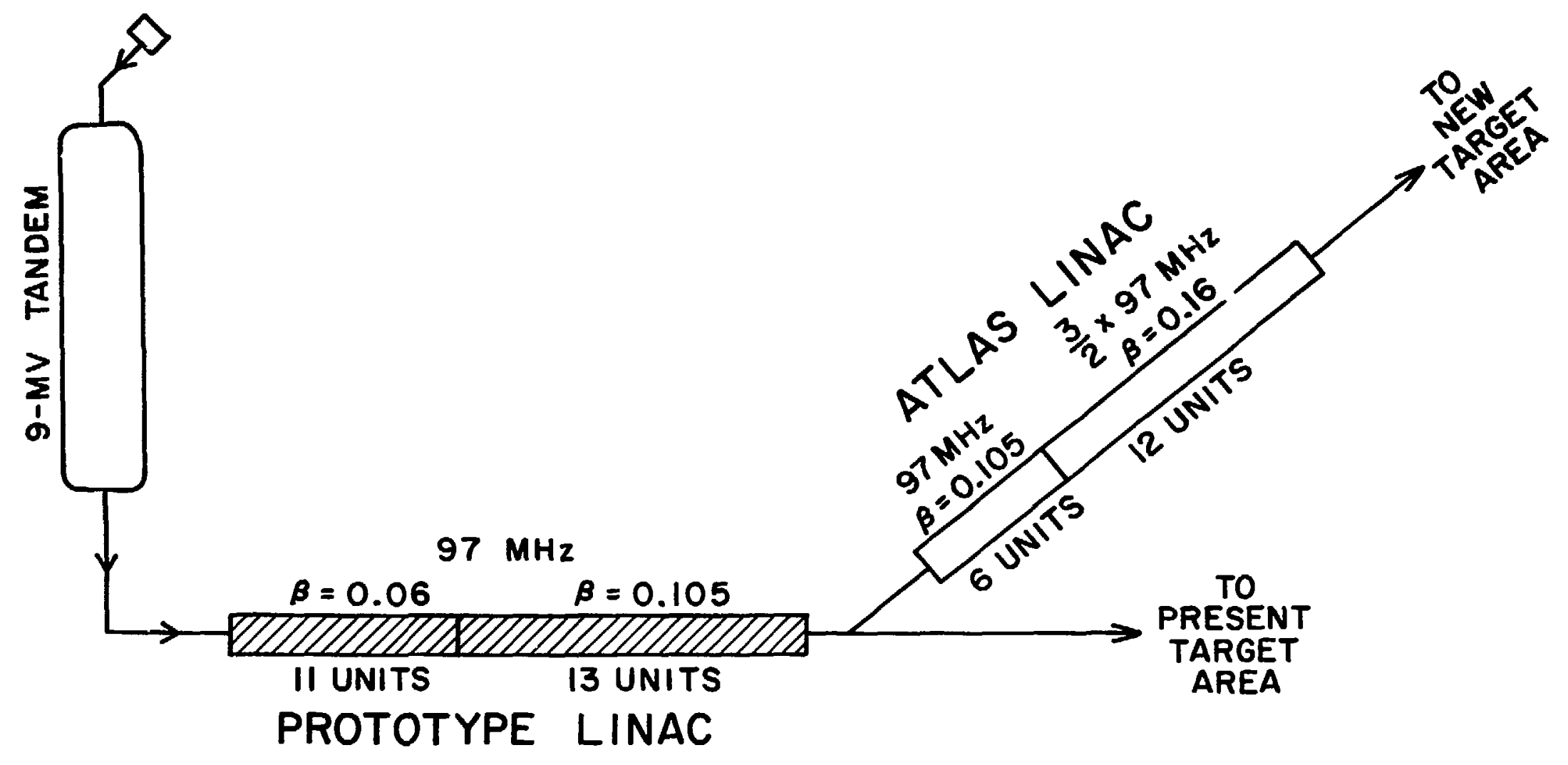

Fig. VI-2. Schematic representation of the ATLAS linac. 
It is also proving possible to get bullding construction off to a very fast start. At the end of the present reporting period (31 March 1982), the design for the first phase of construction has been completed and construction 1s expected to start in May 1982. An artist's conception of the building is shown in Fig. VI-3. Here the monolithic structure on the left is the ATLAS experimental hall, a high-bay area that is connected to the existing accelerator area (on the right) by means of the ATLAS Iinac tunnel.

The ATLAS construction schedule is summarized by Fig. VI-4. Note that, whereas the booster linac was operated almost continuously throughout the resonator-installation process, the ATLAS addition w111 not be used until the Installation of all resonators has been completed in early 1985 . In the meantime, the 24-resonator booster w111 continue to be intensively used for research in the present experimental area. 


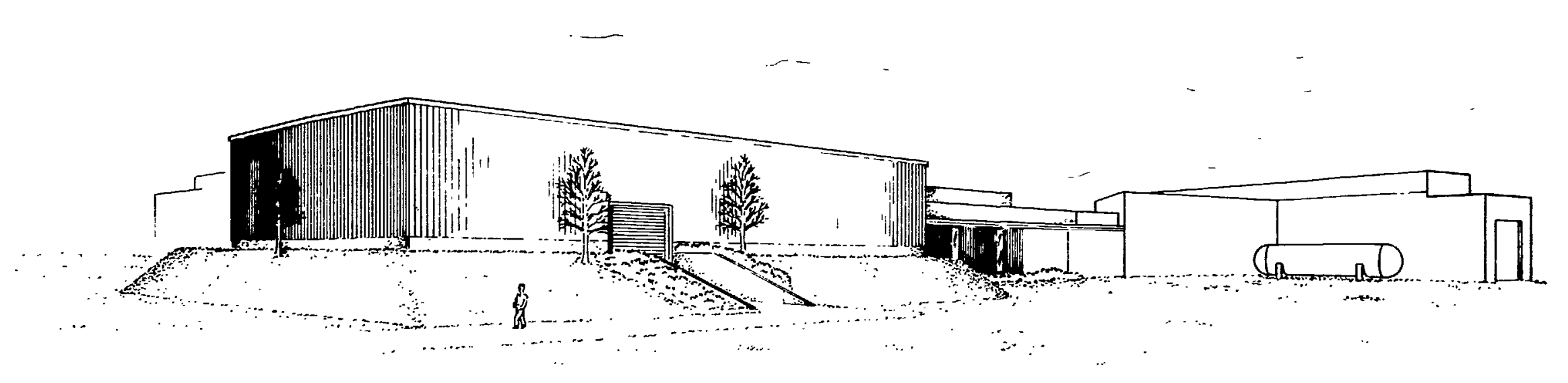

Fig. VI-3. The ATLAS building, as viewed from the north. 


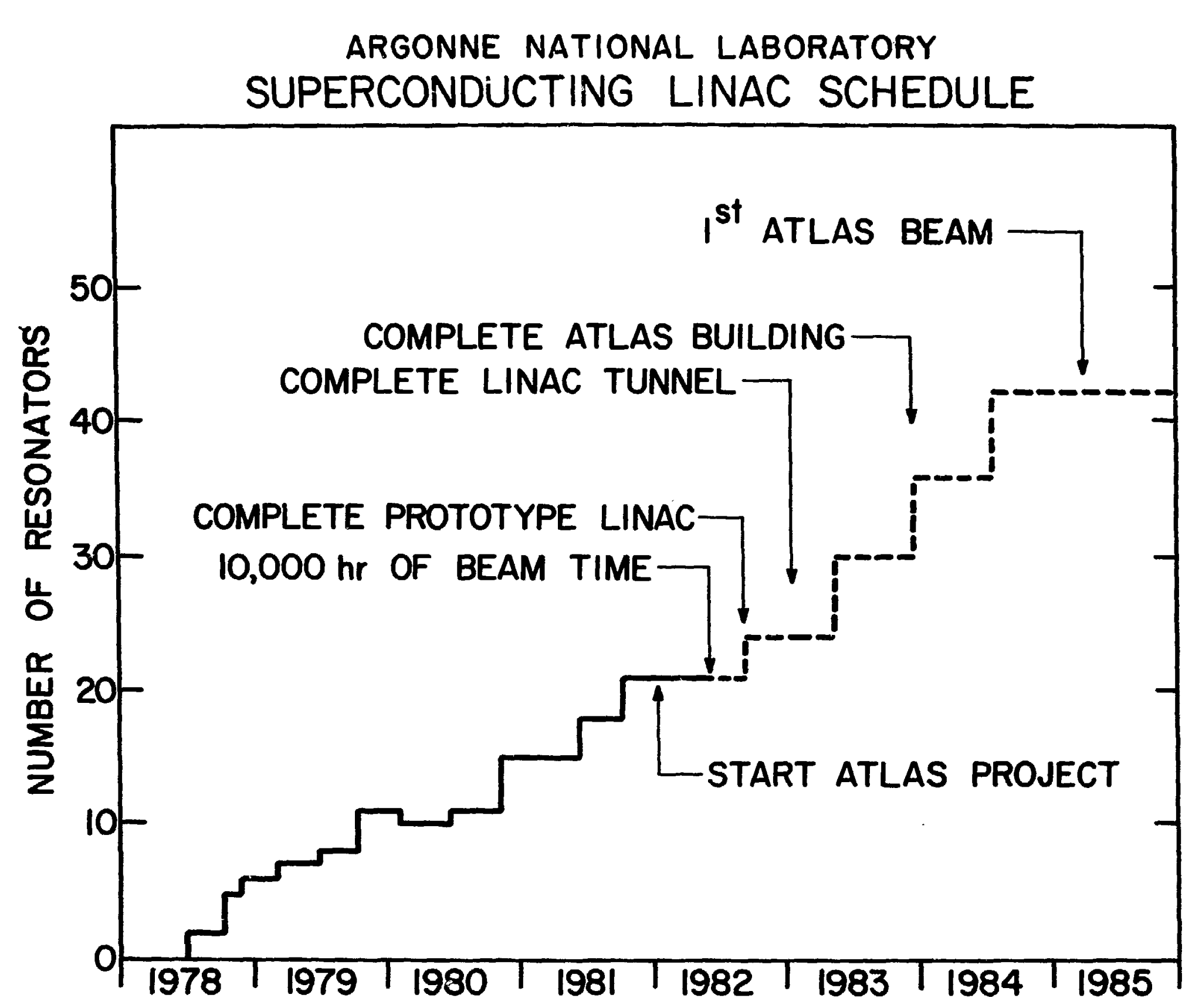

Fig. VI-4. Schedule of critical events in the ATLAS project. 
VII. ACCELERATOR OPERATIONS

\author{
INTRODUCTION
}

This section is concerned with the operation of both the tandemlinac heavy-ion accelerator and the Dynamitron, two accelerators that are used for entirely different research. Developmental activities associated with the tandem and the Dynamitron are also treated here, but developmental activities associated with the superconducting linac are covered separately in Sec. VI, because this work is a program of technology development in its own right. 


\section{A. OPERATION OF THE TANDEM-LINAC ACCELERATOR}

This activity consists of the operation of the tandem-1inac accelerator system so as to provide beams of heavy-ion projectiles for research in several areas of nuclear physics and occasionally in other areas of science. The accelerator system consists of a 9-MV tandem electrostatic accelerator and several sections of a superconducting-linac energy booster. The linac is now (March 1982) about 90\% completed and the tandem-1inac system is now equivalent in energy to a 23-MV (terminal) tandem with two strippers. Completion of the linac is expected late in 1982, at which time the layout of the whole facility will be as shown in Fig. VII-1.

See Sec. VI for information about the technology of the superconducting linac.

\section{OPERATION OF THE ACCELERATOR}

P. K. Den Hartog, R. C. Pardo, C. E. Heath, F. H. Munson, G. P. Zinkann, R. Benaroya, L. M. Bollinger, and K. W. Shepard

The accelerator is now operated almost entirely with the tandem serving as injector of the linac. For only about ten days yearly is the tandem used as a stand-alone machine. This division of time is determined entirely on the basis of the users' choice, not by administrative decision.

During this reporting period, the normal operating schedule of the accelerator system has been from Monday morning to Saturday morning, five whole days each week. This limited operating schedule has had to be imposed, because of limited funding, in spite of ar intense demand for running time. Another aspect of the operation imposed by the available funds has been the need to operate the linac without qualified operators being present during night-time shifts. That is, the accelerator user operates the linac by instructing the control computer and calls for help from a member of the linac-development team only if a serious problem arises. This mode of operation is often satisfactory but it also results in inefficiencies, since the user is not likely to see a problem developing in time to prevent it.

In recognition of the need for more running time and more user assistance, the Laboratory has provided discretionary funds that will permit the operating schedule to be expanded somewhat during the second half of 1982. The present plan is to increase the schedule to six days weekly and, in addition, to have a qualified linac engineer/operator present during the 


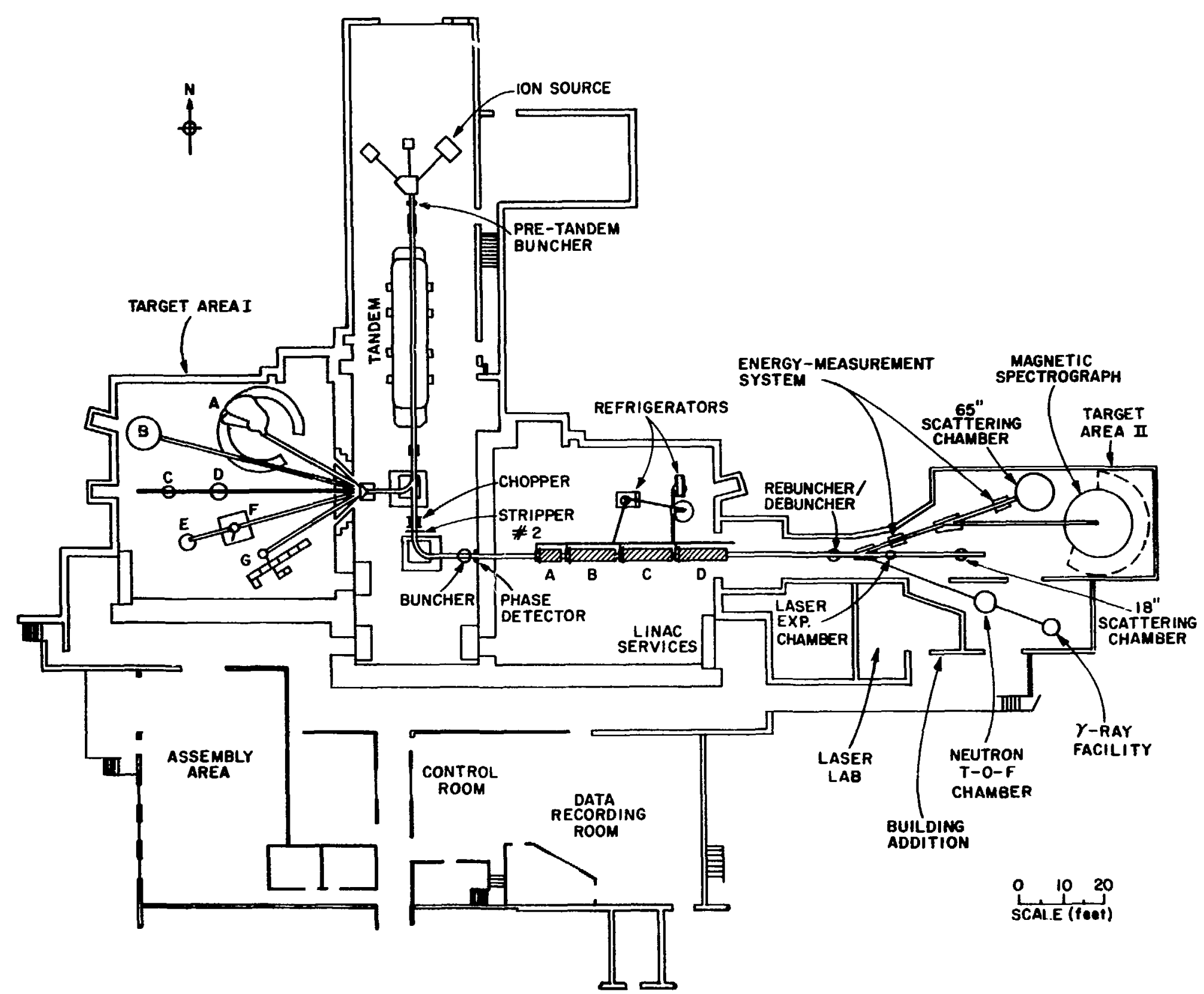

Fig. VII-1. Layout of the present superconducting linac and its experimental area. 
TABLE VII-I. Operation of the ANL tandem-1inac heavy-ion accelerator.

Fiscal Year

$19811982^{*}$

Distribution of Machine Time (Hours)

$\begin{array}{lrr}\text { Research } & 3698 & 3695 \\ \text { Tuning } & 315 & 305 \\ \text { Machine Studies } & 780 & 800 \\ \text { Unscheduled Maintenance } & 525 & 510 \\ \text { Scheduled Shutdown } & \underline{3442} & \underline{3450} \\ \quad \text { Total (1 yr) } & 8760 & \mathbf{8 7 6 0}\end{array}$

Distribution of Research Beam Time (\%)

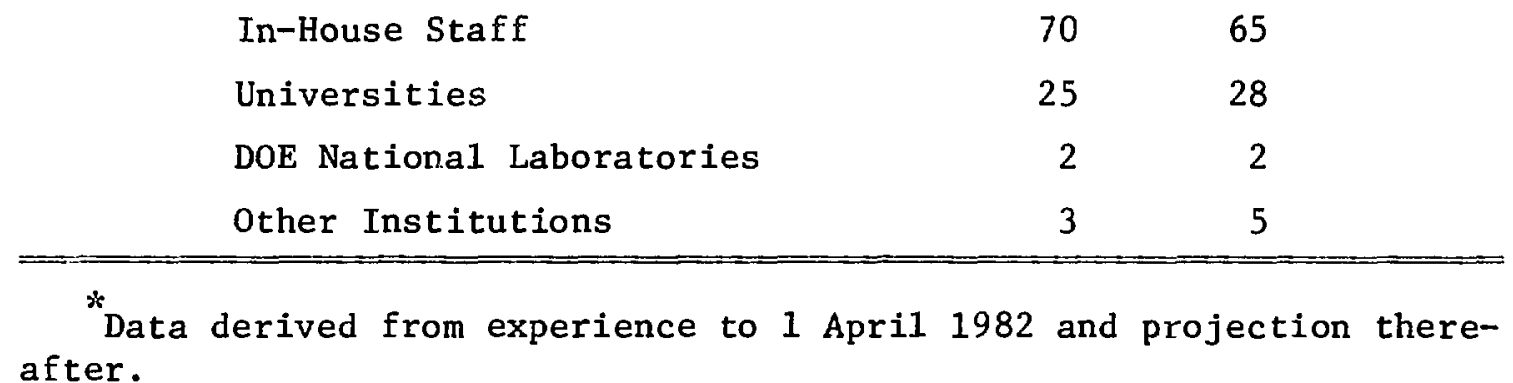

evening shift. These two changes are expected to increase the effective running time to an important extent.

Although the tandem-1inac accelerator is now operated routinely for research most of the time, about $25 \%$ of the possible running time is lost to interruptions caused by various accelerator-upgrading activities and by building-construction activities associated with the ATLAS project (see Sec. VI). This pattern of operation is expccted to persist until ATLAS has been completed.

Some statistics about accelerator-operating experience are given in Table VII-I, and representative beams that have been accelerated are described in Table VII-II. 
TABLE VII-II. Representative beams accelerated by the ANL tandem-linac during 1981. The quantities $\mathrm{q}_{1}$ and $\mathrm{q}_{2}$ are the ion charge states after the terminal stripper and the port-tandem stripper, respectively.

\begin{tabular}{|c|c|c|c|c|}
\hline \multirow[b]{2}{*}{ Ion } & \multicolumn{2}{|c|}{$\begin{array}{l}\text { Charge } \\
\text { state }\end{array}$} & \multicolumn{2}{|c|}{$\begin{array}{l}\text { Beam energy } \\
(\mathrm{MeV}) \text { from }\end{array}$} \\
\hline & $q_{1}$ & $q_{2}$ & Tandem & Linac \\
\hline${ }^{12} \mathrm{C}$ & 5 & 5 & 51.0 & 106 \\
\hline & 6 & 8 & 59.5 & 163 \\
\hline${ }^{30} \mathrm{Si}$ & 7 & 12 & 68.0 & 228 \\
\hline${ }^{32} \mathrm{~s}$ & 8 & 13 & 76.5 & 280 \\
\hline & 8 & 13 & 76.5 & 263 \\
\hline${ }^{35} \mathrm{Cl}$ & 8 & 13 & 76.5 & 207 \\
\hline${ }^{37} \mathrm{Cl}$ & 8 & 15 & 76.5 & 278 \\
\hline${ }^{40} \mathrm{Ca}$ & 9 & 9 & 85.0 & 200 \\
\hline${ }^{40} \mathrm{Ca}$ & 9 & 15 & 85.0 & 289 \\
\hline${ }^{56} \mathrm{Fe}$ & 10 & 19 & 93.5 & 354 \\
\hline${ }^{58} \mathrm{Ni}$ & 10 & 19 & 93.5 & 373 \\
\hline${ }^{60} \mathrm{Ni}$ & 10 & 19 & 93.5 & 343 \\
\hline${ }^{64} \mathrm{Ni}$ & 10 & 19 & 93.5 & 294 \\
\hline
\end{tabular}

2. STATUS OF THE SUPERCONDUCTING LINAC

The fourth and last section of the superconducting prototype linac was installed in June 1981, with 22 of the planned 24 resonators in place.

A11 24 resonators are expected to be operational by the end of 1982 , at which time they will provide about 21 MV of acceleration under routine operating conditions. The combined tandem-linac system will then have an accelerating power aquivalent to that of a 25-MV tandem with two strippers. We expect to increase this accelerating power over the next few years by systematically reducing various known limitations on performance. 
3. UPGRADING OF THE LINAC

J. Aron, ${ }^{*}$ R. Benaroya, L. M. Bollinger, B. E. Clifft, ${ }^{*}$ K. W. Johnson, * P. Markovich, ${ }^{*}$ J. M. Nixon, * R. C. Pardo, K. W. Shepard, and G. P. Zinkann

The pace of development of the prototype linac has slowed down during the past year because the major problems have been solved and the emphasis has now shifted to operation of the accelerator and to the construction of ATLAS. Nevertheless, significant improvements continue to be made.

\section{a. Resonator Fabrication}

The final two resonators for the booster will be completed before summer 1982 and then installed as soon as the linac-operating schedule permits.

\section{b. Computer Control System}

R. C. Pardo

Perhaps the major development of the past year is the completion of a refined, reliable computer-based control system for the linac. This system now permits the linac to be tuned in principle without human intervention and in fact with only minimal help. Consequently, the time required for this complex operation can be less than half an hour (although it is usually longer), and a number of persons with only a minimal understanding of the linac are being trained to do the tuning.

Similarly, the beam energy can now be changed rapidly ( $\sim 1 \mathrm{~min}$ ), and resonator operating parameters can be changed over a wide range without retuning the system. These features make it easy for the inexperienced user to vary the energy and, if necessary, to eliminate the influence of a malfunctioning resonator by changing operating conditions.

These and other developments 1 isted below are expected to improve the operating efficiency of the accelerator steadily during the coming year.

\section{c. Liquid-Nitrogen Distribution}

\section{J. M. Nixon ${ }^{*}$}

The original rather crude liquid-nitrogen distribution system is now being replaced by one that has vacuum-insulated lines and other refinements. 
This improvement, which is being implemented during March 1982, is expected to reduce significantly the costs for liquid nitrogen.

The next step will be to refine the controls on liquid-nitrogen

flow through the linac, again with the objective of reducing operating costs.

\section{d. Slow-Tuner Pressure Controller}

K. W. Shepard

A new type of pressure controller for the slow tuner was put into service in 1981 (see Sec. VI on Superconducting Linac). This new controller has improved the resonator-frequency stability when the resonators are operated for a long time at a constant field, but the pressure range of the controller is not large enough to handle well the sudden large changes in field that of ten occur when the beam energy is changed. Thus, a better controller is needed, and work has started on a concept that appears to meet all requirements.

\section{e. Liquid-Helium Control System}

B. E. Clifft ${ }^{*}$ and K. W. Johnson ${ }^{*}$

We have put into service a simple control system that can control one of several parameters of the liquid-helium system: (1) the liquid level in the storage dewar, (2) the net production rate of liquid helium, or (3) the dewar pressure. This system has made it possible to carry out long and rather complex tasks such as cooldown of the linac without having anyone present most of the time.

\section{f. Heavy-Ion Beam Switch}

B. E. Clifft, ${ }^{*}$ K. W. Johnson, * and R. C. Pardo

A beam sweeper consisting of a pair of deflection plates driven by a square-wave signal has been developed and is being installed. This system is to be used to switch the beam suddenly on and off for variable periods in order to measure relatively long nuclear-decay lifetimes. The voltage pulse on the deflection plates can come full on $(2 \mathrm{kV})$ or full off in 0.1 us at a frequency as high as $500000 \mathrm{~Hz}$. Thus, the system will provide beam pulses

*Chemistry Division, ANL. 
that are short enough to permit the lifetimes of many high-spin states to be measured.

\section{B. Energy-Measurement System}

R. C. Pardo and R. N. Lewis *

A nondestructive energy-measurement system based on a new time-offlight technique has been developed and put into service. Additional information about the technology is given in Sec. VI. The availability of the new energy-measurement system is expected to result in an important improvement in the quality of operation of the tandem-linac accelerator for a number of reasons. In particular, it will allow users to determine and/or to select the beam energy with precision, and unplanned changes in energy will be detected and will warn the operator that there is a problem.

\section{UPGRADING OF THE TANDEM}

P. K. Den Hartog, C. E. Heath, and F. H. Munson

During the past year, the upgrading effort on the tandem has been directed mainly at increasing the number of diagnostic tools and improving the reliability and ease of accelerator operation.

\section{a. Stripping Foils}

P. K. Den Hartog

Studies reported in the preceding Annual Review revealed that significant improvements in stripper-foil lifetimes can be obtained with a new preparation technology. This work has continued during the past year. A chamber for producing hydrocarbon cracked foils and a press for mounting them were designed and constructed. The new types of foils have been used successfully in the Tandem, eliminating foil replacement as the primary cause for entry into the pressure vessel. Work will continue on these and other types of stripping media.

\footnotetext{
*Electronics Division, ANL.
} 


\section{b. Terminal-Control System}

The terminal-control system developed during 1980 has continued to operate reiliably. There have been no failures of the fiber optic links or microcomputers. Improvements designed during the past year will allow more devices to be controlled and monitored.

\section{c. Charging System}

Capacitive pickups were installed on the charging-chain inductors in order to monitor the performance of the charging system. These devices have been useful in diagnosing component deterioration prior to failure, thereby avoiding major damage. Also, a new type of HV pressure feedthrough with arc-discharge protection was installed. This has eliminated a recurring breakdown problem.

\section{d. On-Line Ion Source}

The on-line inverted sputter source has been developed into a very reliable component. A spare source-head and pumpout system was constructed, and this allows a second source always to be ready for use.

\section{e. Beam-Line and Accelerator Control}

Numerous improvements were made in the accelerator and beam-transport control systems. Several obsolete and unreliable components were removed or replaced and the associated control wiring revamped. The infrequently used charge-exchange and direct-extraction injectors were removed in preparation for the installation of the new generation of ion sources which will serve ATLAS.

\section{ION-SOURCE DEVELOPMENT}

P. J. Billquist and J. L. Yntema

The effort devoted to the development of negative-ion sources has been reorganized into a small but coherent program during the past year, and the enhanced emphasis has already led to significant results. 


\section{a. Ion-Source Test Facility}

The main part of a new ion-source test facllity has been completed and put into service. It has a mass resolution of $0.6 \%$, and its injection system allows the use of the proper extraction geometry for several kinds of sources, including the inverted sputter source, the Billen-Richards source (SNICS), and the Aarhus source (ANIS). An emittance-measurement system has been designed and constructed, and it will soon be put into service.

\section{b. New Design for the Inverted Sputter Source}

The inverted Cs sputter source has been redesigned to test some new ideas concerning the optics of the source. The initial results, obtained on the test facility, show that the intensities of negative-ion beams available from the new source are at least five times greater than from the original source for $\mathrm{C}, \mathrm{Al}, \mathrm{Fe}, \mathrm{Ni}$, and $\mathrm{Cu}$. Moreover, the effective source size is exceptionally small ( $<1 \mathrm{~mm}$ diameter), making the new source attractive for use with separated isotopes. In view of these and other improvements, we believe that the new design is a significant step forward, and an effort is being made to install the new source on the tandem as soon as possible.

\section{c. West Injector for the Tandem}

A second ion-source position and injector for use on the tandem has been designed and is being built as part of the ATLAS project. This new injector will be used for two purposes: (1) to carry out on-line tests of new sources and (2) to provide an installed spare source in order to minimize loss of running time caused by source failures.

The redesigned sputter source described in Sec. VII.A5b will be installed inftially on the new injector, where any remaining operational problems can be eliminated without seriously interrupting routine operation of the accelerator.

\section{d. The Aarhus and SNICS Sources}

The Aarhus and SNICS sources have both been studied on the test bench, and satisfactory operation has been achieved for several ions. The sources will be put into operation on the tandem when the second injector is avai1able. 


\section{NEAR-TERM PLANS}

\section{a. Accelerator Operation}

The tandem-1inac system will continue to be operated routinely for physics research during the coming year. From now on, relatively little downtime will be required for additions to or upgrading of the present accelerator, but several blocks of downtime must be scheduled to accommodate various construction activities required by the ATLAS project.

\section{b. Booster Improvements}

The final two rasonators of the booster linac will be put into operation in late 1982, thus sompleting the booster in all respects. This work and the activation of several other resonators that are installed but not working will extend by about $20 \%$ the energy range accessible for many ions.

A second major task for the coming year is the development and installation of a good pressure controller for the slow tuners. This work is expected to lead to much more reliable operation.

\section{c. Tandem Improvements}

The main task for the coming year is to install and place in operation a terminal lens and its control system. This work is aimed at increasing the beam transmission of the tandem, especially for projectiles with $A \geq 60$.

\section{d. Ion-Source System}

The new West Injector will be completed at least to the point of being operational during the coming year, and will be used for on-line tests of the improved Inverted sputter source described in Sec. VII.A5b. If these tests are successful, the source (which is still in an experimental stage) will occasionally be used to provide beams for research. 


\section{ASSISTANCE TO OUTSIDE USERS OF THE TANDEM-LINAC ACCELERATOR}

Outside use of the tandem-linac accelerator has increased substantially during the past year, both in terms of the number of persons involved and the total amount of running time provided. This trend is expected to continue during the coming year for several reasons: (1) the unique capabilities of the accelerator for some kinds of research, (2) the facts that the accelerator operation is now largely routine and the experimental system is well developed, and (3) the delays in completion of other new heavy-ion accelerators.

Several changes that will substantially influence the operation of the tandem-linac accelerator are now being implemented. One is the appointment of an Advisory Committee on the scheduling of research time. The initial major task for the committee will be to advise the Laboratory regarding policy in the allocation of runing time,

A second important change is a plan to increase the scheduled running time from five days to six days weekly and simultaneously to increase the quantity and quality of operating assistance to accelerator users.

These improvements are being instituted at this time principally for the benefit of outside users. The physical arrangements available to outside users are also being steadily upgraded to include office space and bunk-room facilities near the accelerator.

The magnitude of the outside use of the accelerator may be judged from the following two lists: (a) the experiments performed and (b) the universities represented. As may be seen from the names associated with each experiment, the university groups are by now playing a major role in an important fraction of the experiments and a dominant role in some.

\section{a. Experiments Involving Outside Users}

All experiments in which outside users participated during the period April 1981 to March 1982 are listed below. The names in parentheses are Argonne collaborators.

(1) ${ }^{40} \mathrm{Ca}+{ }^{40} \mathrm{Ca}$ : Structure in the Energy Spectra; Z, A Distributions; Coincident Light Particles $(p, d, t, \alpha) ; x-y$ Gas Counter Development A. C. Mignerey, H. Breuer, A. Gokmen, and S. Barkaw, University of Maryland; V. Viola, K. Kwiatkowski, and S. Zhou, Indiana University; B. Jacak, W. Lynch, B. Tsang, Xu Zurun, C. Gelbke, and Z. Konig, Michigan State University; P. A. Baisden, Lawrence Livermore Laboratory (K. Wolf, I. Ahmad, B. Glagola, S. Saini, J. Yntema, and P. J. Billquist)

(2) Development of Laser Spectroscopy Experimental Program M. A. Finn, G. W. Greenlees, and S. L. Kaufman, University of Minnesota; R. M. Evans and D. A. Lewis, Iowa State University (C. Davids) 
(3) (a) Evaporation Residue Studies of ${ }^{32} \mathrm{~S}+112,116,120,124 \mathrm{Sn}$

(b) Fusion-Fission Studies of $58 \mathrm{Ni}+116,124 \mathrm{sn}, 154 \mathrm{Sm}, 170 \mathrm{Yb}$

(c) Evaporation Residue Studies of $58 \mathrm{Ni}$ on Targets of Even Tin Isotopes F. W. Prosser, Jr., and R. A. Racca, University of Kansas (W. Henning, H. Ernst, W. Freeman, T. Humanic, D. Geesaman, W. Kühn, and J. P. Schiffer)

(4) Recoil Distance Lifetime Measurement in ${ }^{154} \mathrm{Dy}$

S. Faber, P. J. Daly, H. Helppi, A. Pakkanen, and Y. H. Chung, Purdue Uriversity (T. L. Khoo, I. Ahmad, P. Chowdhury, and Z. Grabowski)

(5) Spectroscopy of the $\mathrm{N}=82$ Nuclei ${ }^{150} \mathrm{Er}$ and ${ }^{151} \mathrm{Tm}$ from Reactions Induced by $58,60 \mathrm{Ni}$

P. J. Daly, Y. H. Chung, H. Helppi, and A. Pakkanen, Purdue University (T. L. Khoo, I. Ahmad, P. Chowdhury, and Z. Grabowski)

(6) ${ }^{12} \mathrm{c}\left({ }^{16} 0, \alpha\right)$ Studies and Search for Massive Transfers R. E. Segel and S. Mukhopadhyay, Northwestern University; H. T. Fortune, L. C. Bland, M. A. Carchidi, P. H. Kutt, and G. Stephans, University of Pennsylvania (W. Henning)

(7) Width of Fission Mass Distribution in the ${ }^{32} \mathrm{~S}$ on ${ }^{208} \mathrm{~Pb}$ Reaction C. K. Gelbke, W. G. Lynch, Zurun Xu, and D. A. Ardouin, Michigan State University; P. A. Baisden and M. A. McMahan, Lawrence Livermore Laboratory (B. Back, B. Glagola, and S. Saini)

(8) Angular Momentum Effects in the Neutron Spectra from the ${ }^{64} \mathrm{Ni}+{ }^{92} \mathrm{Zr}$ Reaction

F. Haas, J. Kasagi, and R. M. Ronningen, Michigan State University

(I. Ahmad, P. Chowdhury, R. Janssens, T. L. Khoo, and W. Kühn)

(9) Yrast Population Patterns in a Wide Range of Nuclei

P. J. Daly, Y. H. Chung, M. D. Kortelahi, and Z. Grabowski, Purdue University (T. L. Khoo, I. Ahmad, P. Chowdhury, and R. Janssens)

(10) Detector Tests

B. W. Wehring and G. M. Swift, University of Illinois (B. Glagola,

J. Gindler, and B. Wilkins)

(11) Accelerator-Based Mass Spectroscopy Studies

B. Myslek-Laurikainen, Institute of Nuclear Research, Swierk,

Poland (W. Kutschera, W. Henning, R. K. Smither, and J. L. Yntema)

(12) Ni-Induced Multineutron-Pair Transfers on Sn Targets

S. Lunardi and A. Stefanini, University of Padova, Italy

(W. Henning and W. Kutschera)

(13) Fusion Angular Distribution Measurements

K. Cassidy, Dartmouth College (B. Back, R. Betts, B. Glagola, and

J. Lebowitz) 
(14) Energy Dependence of ${ }^{24} \mathrm{Mg}+{ }^{24} \mathrm{Mg}$ Elastic Scattering and Reactions F. Haas, Michigan State University; F. W. Zurmuh?e, University of Pennsylvania (S. Saini, R. R. Betts, I. Ahmad, B. B. Back, B. G. Glagola, and J. L. Yntema)

(15) Lifetime Measurements in ${ }^{153}$ Ho D. C. Radford and M. S. Rosenthal, Yale University; S. Faber, Purdue University (T. L. Khoo and P. Chowdhury)

(16) Lifetime of Continuum States by a Doppler Shift Attenuation Method H. R. Andrews, D. Ward, Chalk River Laboratory, AECL; S. Faber, Z. Grabowski, P. Daly, A. Pakkanen, H. Helppi, J. Wilson, and Y. H. Chung, Purdue University (P. Chowdhury, T. L. Khoo, and I. Ahmad)

(17) (a) $\gamma-\gamma$ Coincidence Measurements in ${ }^{153}$ Dy

(b) Angular Distribution for $\gamma$ Rays Deexciting High Spin States in ${ }^{150} \mathrm{Er}$

(c) High Spin States in $186 \mathrm{Hg}$

M. O. Kortelahti, P. J. Daly, Y. H. Chung, and Z. W. Grabowski, Purdue University (R. Janssens, P. Chowdhury, I. Ahmad, and T. L. Khoo)

\section{b. Outside Users and Institutional Affiliations}

The following list gives the names and institutional affiliations of outside users of the tandem-linac system during the period April 1981 through March 1982 .

(1) Chalk River Nuclear Laboratories, A.E.C.L.

H. R. Andrews

D. Ward

(2) University of Copenhagen

J. Borggreen

G. Sletten

(3) Dartmouth Co11ege

K. Cassidy

(4) Hebrew University (Jerusalem)

M. Pau1

(5) University of Illinois (Urbana)

G. M. Swift

B. W. Wehring

(6) Indiana University

K. Kwiatkowski

V. E. Viola

(7) Iowa State University

R. M. Evans

D. A. Lewis 
(8) University of Kansas

F. W. Prosser, Jr.

R. A. Racca

(9) Lawrence Livermore Laboratory

P. A. Baisden

M. A. McMahan

(10) University of Maryland
S. Barkan
H. Breuer
A. Kokman
A. C. Mignerey

(11) Michigan State University
D. A. Ardouin
C. K. Gelbke
F. Haas
B. Jacak
J. Kasagi
Z. Konig
W. G. Lynch
R. M. Ronningen
B. Tsang
Xu Zurun

(12) University of Minnesota

M. A. Finn

(13) Northwestern University

S. Mukhopadhyay

R. E. Segel

(14) University of Padova

S. Lunardi

S. Stefanini

(15) University of Pennsylvania

L. C. Bland

M. A. Carchidi

K. Daneshvar

H. T. For tune

P. H. Kutt

G. Stephans

R. W. Zurmuhle

(16) Purdue University

Y. H. Chung

P. J. Daly

S. Faber

H. Helppi

M. O. Kortelahti

A. Pakkanen

J. Wilson 
(17) Institute for Nuclear Research, Swlerk, Poland

B. Myslek-Laurikainen

(18) Yale University

D. C. Radford

M. S. Rosenthal

S. J. Sanders

c. Summaries of Major User Programs

Several groups listed in the above tables have embarked on extensive research programs that are likely to make use of the tandem-1inac accelerator for several years. In order to give some indication of the extent of these efforts, the following descriptions summarize the research of four groups that have been most active during the period April 1981 to March 1982.

\section{(i) The Purdue University Group}

P. J. Daly, Z. Grabowski, Y. H. Chung, S. R. Faber, H. Helppi,

M. Kortelahti, A. Pakkanen, J. McNeill, and J. Wilson

The Purdue University group has an active program on high-spin nuclear states at the linac. The personnel currently includes two professors, one postdoc, and four graduate students. One student whose thesis was largely based on work conducted at the linac has already graduated. A postdoctoral fellow was based at Argonne for about 18 months and two sabbatical visitors from Finland, who have recently returned, were also actively engaged in this effort.

The program uses in-beam $\gamma$-ray techniques and is directed at several aspects of nuclear structure at high spin. We are testing the validity of the $Z=64$ subshell closure through spectroscopic studies of $N=82$ nuclei close to the proton drip line. By studies on $N=87$ and 88 Dy isotopes we are also investigating the transition between oblate aligned-particle and prolate-collective structures as a function of both spin and neutron number. In addition we are also studying the excited states which lie above the yrast lines of nuclei near ${ }^{152}$ Dy.

The program is conducted in close collaboration with the $\gamma$-ray group at Argonne and details may be found elsewhere in this document.

We have begun a project to construct a superconducting solenoid lens to be used as a conversion electron spectrometer. The solenoid is now being manufactured by a firm in England and other parts are being ordered. The instrument will be assembled and tested at Purdue, and then installed at Argonne in summer 1983. 


\section{(ii) University of Kansias Collaboration}

F. W. Prosser, Jr., and R. A. Racca

During the past year our group has participated in a series of measurements of fusion-evaporation cross sections for beams of ${ }^{32} \mathrm{~s}$ and ${ }^{58} \mathrm{Ni}$ on targets of several $\mathrm{Sn}$ isotopes, from ${ }^{112} \mathrm{Sn}$ to ${ }^{124} \mathrm{Sn}$. We were also involved in measurements of fusion-fission cross sections with ${ }^{58} \mathrm{Ni}$ beams on targets from ${ }^{116} \mathrm{Sn}$ to ${ }^{170} \mathrm{Yb}$. In addition, much of the year was spent in completing the analysis of gamma-ray measurements of the fusion of $16,18 \mathrm{O}+24,26 \mathrm{Mg}$. These measurements, together with the earlier work on ${ }^{15} \mathrm{~N}+{ }^{27} \mathrm{Al}$ made up the experimental basis for the thesis of $\mathrm{R}$. A. Racca which was defended in January 1982. In the immediate future, continuation of further work necessary to complete the ${ }^{32} \mathrm{~S}$ and ${ }^{58} \mathrm{Ni}$ experiments is planned as well as an extension of fusion-evaporation measurements to heavier targets, to better understand the disappearance of this reaction mechanism in heavier systems. Also there is interest to use the higher energy available with ATLAS to study the importance of incomplete fusion in lighter systems, such as ${ }^{16} \mathrm{O}+{ }^{40} \mathrm{Ca}$.

\section{(iii) MSU and LLL Collaboration}

C. K. Gelbke, W. Lynch, M.-Y. B. Tsang, Z. Xu, P. Baisden, and M. McMahan

Recent measurements of fission decay of nuclei with vanishing liquid-drop fission barriers have beer. reported in the literature. These experimental results show a rapidly increasing width of the fission fragment mass distribution and have suggested possible existence of a fast fission process. We have studied the fission decay of ${ }^{240} \mathrm{Cf}$ produced by bombarding a ${ }^{208} \mathrm{~Pb}$ target with ${ }^{32} \mathrm{~S}$. Fission fragment half-widths and angular distributions were measured as a function of bombarding energy from 180 to $270 \mathrm{MeV}$. The measurements of the fission fragment mass widths were performed with a time-of-flight telescope in coincidence with a position-sensitive solid state detector. By using the fission fragment folding angle information the separation of the fission decay of the compound nucleus from the fission decay of target residues following transfer reactions will be possible. The experimental results will provide more definitive information concerning the properties of rotating nuclel with vanishing liquid drop fission barrier for which the concept of a compound nucleus should not apply. 


\section{(iv) Iowa State and Minnesota Collaboration}

D. A. Lewis, R. M. Evans, G. W. Greenlees, S. L. Kaufman, and M. A. Finn

This project will use on-line laser spectroscopy to study the optical hyperfine structure of radioactive atoms. The objective is to extract information on spins, moments, and the variation of charge radii for ground states and isomers. The species under investigation will be produced by heavy-ion beams from the tandem-linac. The radioactive atoms recoil from the production target, become thermalized in a helium atmosphere, and then are transported by a liquid-nitrogen-cooled helium jet to the laser interaction region. Resonance fluorescence spectroscopy will be employed to observe the optical transitions. Essentially Doppler-free linewidths will be obtained by collimating the atoms into an atomic beam as they emerge from the helium jet. Two cooled photomultiplier tubes will detect the fluorescent light.

In the past year the cryogenic helium jet has been tested using radioactivities produced on line at the tandem. The laser system has been set up in the new laser laboratory, and is now undergoing tests using stable atomic beams. A target station on the tandem-linac beamline has been constructed, and on-line tests with the laser coupled to the helium jet will be conducted in the near future. 


\section{B. OPERATION OF THE DYNAMITRON FACILITY}

The Physics Division operates a high-current 4.5-MV Dynamitron accelerator which has unique capability as a source of ionized beams of most atoms and many molecules. Among the unusual facilities associated with the Dynamitron are (1) a beam line capable of providing "supercollimated" ion beams permitting angular measurements to accuracies of 0.005 degree, (2) a beam-foil measurement system capable of measuring lifetimes down to a few tenths of a nanosecond, (3) an experimental system dedicated to measuring absolute nuclear cross sections at low energy, (4) a varlety of experimental apparatus for weak-interaction studies, (5) a simultaneous irradiation system by which heavy ions from the Dynamitron and helium ions from a 2-MV Van de Graaff accelerator are focused on the same target, and (6) a postacceleration chopper system giving beam pulses of variable width from about one nanosecond to the millisecond range at repetition rates variable up to $8 \mathrm{MHz}$. A PDP-11/45 computer system is used for on-1ine data analysis and for the control of experimental systems.

\section{OPERATIONAL EXPERIENCE}

A. J. Elwyn, R. L. Amrein, and A, E. Ruthenberg

Overall, the Dynamitron continued to perform well during the past year. The normal operating schedule was twenty-four hours a day, five days a week. Very little running was done on weekends during calendar year 1981. During the year the accelerator was staffed a total of 5344 hours. Of this time 3960 hours (74\%) were scheduled for experimental research during which a beam was provided to the experimenters $78 \%$ of the time. Machine preparation time used up $7 \%$ of the scheduled research time and machine malfunctions the remaining 15\%. Scheduled accelerator improvements (including the upgrading program) and modifications used a total of 1384 hours or $26 \%$ of the total available time.

The great versatility of the Dynamitron continued to be exploited by the research staff. Ion currents on target varied from less than a nanoampere to about 40 microamperes with ion energies ranging from 0.4 to 4.6 MeV. A wide range of both atomic and molecular ions was delivered on target. They included ${ }^{1} \mathrm{H}^{+},{ }^{1} \mathrm{H}_{2}+,{ }^{2} \mathrm{H}^{+},{ }^{1} \mathrm{H}_{3}+,{ }^{3} \mathrm{He}^{+},{ }^{2} \mathrm{H}_{2}+,{ }^{4} \mathrm{He}^{+},{ }^{4} \mathrm{HeH}^{+}$, ${ }^{2} \mathrm{H}_{3}{ }^{+},{ }^{7} \mathrm{Li}^{+},{ }^{20} \mathrm{Ne}^{++},{ }^{12} \mathrm{C}^{+},{ }^{14} \mathrm{~N}^{+},{ }^{20} \mathrm{Ne}^{+},{ }^{14} \mathrm{~N}_{2}{ }^{+},{ }^{12} \mathrm{C}^{16} \mathrm{O}^{+}, 16 \mathrm{O}_{2}{ }^{+},{ }^{35} \mathrm{Cl}^{+},{ }^{40} \mathrm{Ar}^{+}$, ${ }^{58} \mathrm{Ni}^{+},{ }^{84} \mathrm{Kr}^{+}$.

During the year a total of 50 investigators used the Dynamitron in some phase of their experimental research. Of these, 19 were from the 
Physics Division, 13 were from other Argonne research divisions, 14 were outside users from other research facilities, and 4 were members of the Resident Graduate Student Program. Of the scheduled time, $71 \%$ went to members of the Physics Division, $25 \%$ to other Argonne divisions, and $4 \%$ was exclusively assigned to outside users. However, outside users collaborated in experiments that used $43 \%$ of the total available time, and participants in the Resident Graduate Student Program worked on experiments that used $30 \%$ of the time.

At the start of 1981 , the machine upgrading program was in progress. Most of the major modifications (as described in last year's report) and various machine tests were completed by the end of November, 1980. Schedu1ing for experiments started in December, 1980, and the machine has been heavily used by several research groups during 1981 .

Overall, the rebuilt accelerator has been operating with only routine maintenance problems and relatively free of sparking at energies up to $4 \mathrm{MV}$. Above $4 \mathrm{MV}$, however, sparking problems still occur so that operation near $5 \mathrm{MV}$ on the terminal has not as yet been possible. Even so, the machine has on at least one occasion been run successfully at $4.6 \mathrm{MV}$ for a number of hours.

Sparking in the high-voltage terminal near the new air-core capacitor (which was added in order to reduce the $120 \mathrm{kHz}$ rf ripple on the terminal to a negligible value, and thereby improve the energy resolution of the machine) appeared to be one limitation on the maximum at tainable machine energy. However, upon removal of this capacitor, considerable sparking still occurred. It was observed between various components attached to the accelerator tube and the lucite control rods (which are used to operate the variacs controlling the several ion-source power supply voltages in the high-voltage terminal), and it caused severe damage to the rods and prevented operation at higher voltages. The rods are 14 years old and have deteriorated, which may be a factor in their misbehavior. Improvement in the method of fastening control rods together to eliminate the metal setscrews, as well as the reinstallation of the column resistors with spark gaps, seems to have alleviated some of the sparking problems. These resistors serve to prevent the development of excessive voltage differences between the accelerator tube and the nearby column structures surrounding the tube. However, the firing of the resistor spark gaps in parallel with the 
resistors may occur and thus prevent the attainment of terminal voltages near $5 \mathrm{MV}$. Ultimately these resistors may have to be removed or modified. Alternatively one may prefer to delete the existing lucite rods, and replace them by an ion-source control system based on the recently installed fiber optics light-link. Further refinements in the electrical configuration associated with the added terminal (ripple-controlling) capacitor are expected to help reduce sparking in the terminal. Step-by-step improvements to the various machine components and configurations will be made as tests of the maximum voltage-holding capability continue. Routine operation at $5 \mathrm{MV}$ may not be possible, however, until other improvements (such as replacement and modification of the accelerator tube and/or replacement of the present solid-state rectifiers) are made.

In April, the fiber light-link system for monitoring electrical parameters within the high-voltage terminal was badly damaged by a particularly violent series of sparks. We believe that the damage was due to an unsuitable black plastic zipper-tubing used to sheath the fiber bundle within the accelerator. At present these light pipes are being replaced as the various new components become available. No sheathing will be used. is is hoped that the actual monitoring of electrical parameters in the terminal becomes established and standard during the year.

The development of ion sources to provide ion species of interest to specific experimental groups has proceeded slowly during the year because of other projects and commitments. Efforts to obtain a beam of $\mathrm{LiH}^{+}$ions by modification of a standard rf ion source look promising. High intensity beams of light ions at energies below $1 \mathrm{MeV}$ have been obtained by another modification of an rf source, and by improving the ion-optical coupling of the source with the accelerator tube. Proton beam currents of over 100 uamps at energies below $1 \mathrm{MeV}$ have now been achieved.

\section{UNIVERSITY USE OF THE DYNAMITRON}

A. J. Elwyn

The Argonne Dynamitron continues to be a valuable research facility for scientists from outside Institutions. It is not only the accelerator itself that attracts outside investigators but also the unique associated 
experimental equipment as well as the ongoing research program being conducted at the Dynamitron.

Most visiting scientists chose to collaborate with local investigators on problems of common interest. A few, however, worked as an independent group. Some came for a one-time-only experiment, but most are participants in research programs that have spanned a period of several years. During the year fifteen scientists came from nine outside institutions to use the Dynamitron. They came from six states and two foreign countries. They participated in experiments that used $43 \%$ of the time scheduled for research. A list of those institutions from which users of the Dynamitron came during 1981 is given below. The list includes the name of the institution, the title of the research done, and the names of the principal investigators. The names of their Argonne collaborators are enclosed in parentheses.

(1) Fudan University, Shanghai

Influence of Rydberg States in Convoy Electron Measurements

Gu Yuan Zhuang (D. S. Gemmell, E. P. Kanter, D. Schneider, and B. J. Zabransky)

(2) Marquette University

Radiation Damage of Covalent Crystal Structures

L. Cartz, R. Fournelle, A. Gowda, F. G. Karioris, J. C. Labbe, and G. Sarker

(3) Northwestern University

Direct Capture in the ${ }^{27} \mathrm{Al}(\mathrm{p}, \gamma)$ and ${ }^{19} \mathrm{~F}(\mathrm{p}, \gamma)$ Reactions

R. E. Segel, E. Zupranska, G. Hardie, * and M. Wiescher*

(A. J. Elwyn and W. Ray)

(4) Notre Dame University

Spectra of High Spin States in Light Elements

A. E. Livingston (H. G. Berry, R. Brooks, J. Hardis, and

W. Ray)

(5) Ohio State University

Direct Capiure in the ${ }^{27} \mathrm{Al}(\mathrm{p}, \gamma)$ and ${ }^{19} \mathrm{~F}(\mathrm{p}, \gamma)$ Reactions

M. Wiescher, G. Hardie, ${ }^{\text {R. E. Segel, }}$, and E. Zupranska*

(A. J. Elwyn and W. Ray)

(6) Stanford University

Measurement of the Beta Decay of ${ }^{16} \mathrm{~N}\left(0^{-}, 120 \mathrm{keV}\right)$

S. J. Freedman (C. A. Gagliardi, G. T. Garvey, R. D.

Mckeown, B. Myslek-Laurikainen, and A. R. Heath)

*From another outside institution. 
(7) Valparaiso University

The $7_{\mathrm{Li}}(\mathrm{d}, \mathrm{p})^{8} \mathrm{Li}$ Reaction and Solar Neutrino Capture Rates

D. Koetke (B. Filippone and A. J. Elwyn)

(8) Weizmann Institute

Influence of Rydberg States in Convoy Electron Measurements

Z. Vager (D. S. Gemmell, E. P. Kanter, D. Schneider, and

B. J. Zabransky)

(9) Western Michigan University

Direct Capcure in the $27 \mathrm{Al}(\mathrm{p}, \gamma)$ and ${ }^{19} \mathrm{~F}(\mathrm{p}, \gamma)$ Reactions

G. Hardie, R. E. Segel, ${ }^{*}$ M. Wiescher, ${ }^{*}$ and E. Zupranska*

(A. J. Elwyn and W. Ray)

The Resident Graduate Student Program is open to students that

have finished their course work and passed their prelims. They come to Argonne and perform their Ph.D. thesis research here. Five members of this program worked at the Dynamitron during 1981. Altogether they participated in experiments that used $30 \%$ of the time allotted to research. Those who used the accelerator are listed below, together with their home university and their local thesis advisor.

(1) P. W. Arcuni, University of Chicago

H. G. Berry, advisor

(2) A. R. Heath, University of Chicago

G. T, Garvey, advisor

(3) B. Filippone, University of Chicago

C. N. Davids, advisor

(4) C. A. Gagliardi, Princeton University

G. T. Garyey, advisor

(5) J. Hardis, University of Chicago

H. G. Berry, advisor

In addition, the following undergraduate students have participated in research based at the Dynamitron.

(1) J. J. Hofmann, East Stroudsburg State College

D. Schneider, advisor

(2) E. A. Johnson, Washington \& Lee University

D. S. Gemmell, advisor

*From another outside institution. 


\title{
VIII. GeV ELECTRON MICROTRON
}

\author{
INTRODUCT ION
}

There is a widespread conviction in the nuclear physics community that research with electron beams in the GeV range will play a vital role in future progress in fundamental nuclear physics. Support is growing for the construction of a national facility capable of furnishing three beams with a 100\% duty factor. Traditionally the small duty factor and relatively poor beam quality which characterize avallable electron accelerators have been a continuing limitation on research capability. However, recent developments in accelerator technology indicate that a high performance $\mathrm{GeV} \mathrm{C} . \mathrm{W}$. electron accelerator can now be constructed at acceptable cost. At Argonne appropriate resources exist to desigls, construct and operate a national electron accelerator facility in the most cost effective way. An accelerator complex, formerly the Zero Gradient Synchrotron (ZGS) Facility, whose replacement cost is in excess of $\$ 50 \mathrm{M}$, is currently unoccupied and available. There exists an in-house research group which can provide the sclentific planning to insure a successful research program. An ANL study group has studied possible accelerator technologies which could be utilized to meet these needs. They envisaged a $\mathrm{GeV}$ electron accelerator and ancillary experimental areas as a national research facility. It will be a multibeam electron accelerator furnishing two or more intense beams of high-energy $\epsilon$ lectrons simultaneously to highresolution spectrometers and other experiments in nuclear science. Because of superior beam quality and low capital and operating costs, Argonne proposes a three-sided microtron (hexatron), shown schematically in F1g. VIII-1, as the accelerator system to be used to generate the electron beams. The design is constrained to be compatible with the zGS ring building. The use of ZGS structures will result in a mafor reduction in the capltal cost of the facllity. The unlque capabilities of the hexatron will Include the ablifty to deliver continuous beams, with excellent energy resolution, and high intensity. On demand, up to three extracted beams w111 be avallable with Individually varlable energles. The beams will be delivered to three experimental areas which will provide experimental facilities for high-resolution electron scattering studles, medium resolution colncidence experiments, and measurements requiring monochromatic or bremsstrahlung photon beams.

This electron facility is a key element in the national plan for nuclear physics. It will be the first major high-energy electron facility capable of simultaneously serving more than one experimental area. The avallabllity of electron beams with varlable energles in the GeV range, $100 \%$ duty factor, and currents of 100 uamps, will open a new and exciting range of electron nuclear research which will address many of the most Interesting guestions at the frontiers of nuclear physics. Studies w111 be possible of nuclear phenomena at short distance which will reveal the relationship between the internal structure of constituent protons and 


\section{GeV ELECTRON MICROTRON-GEM}

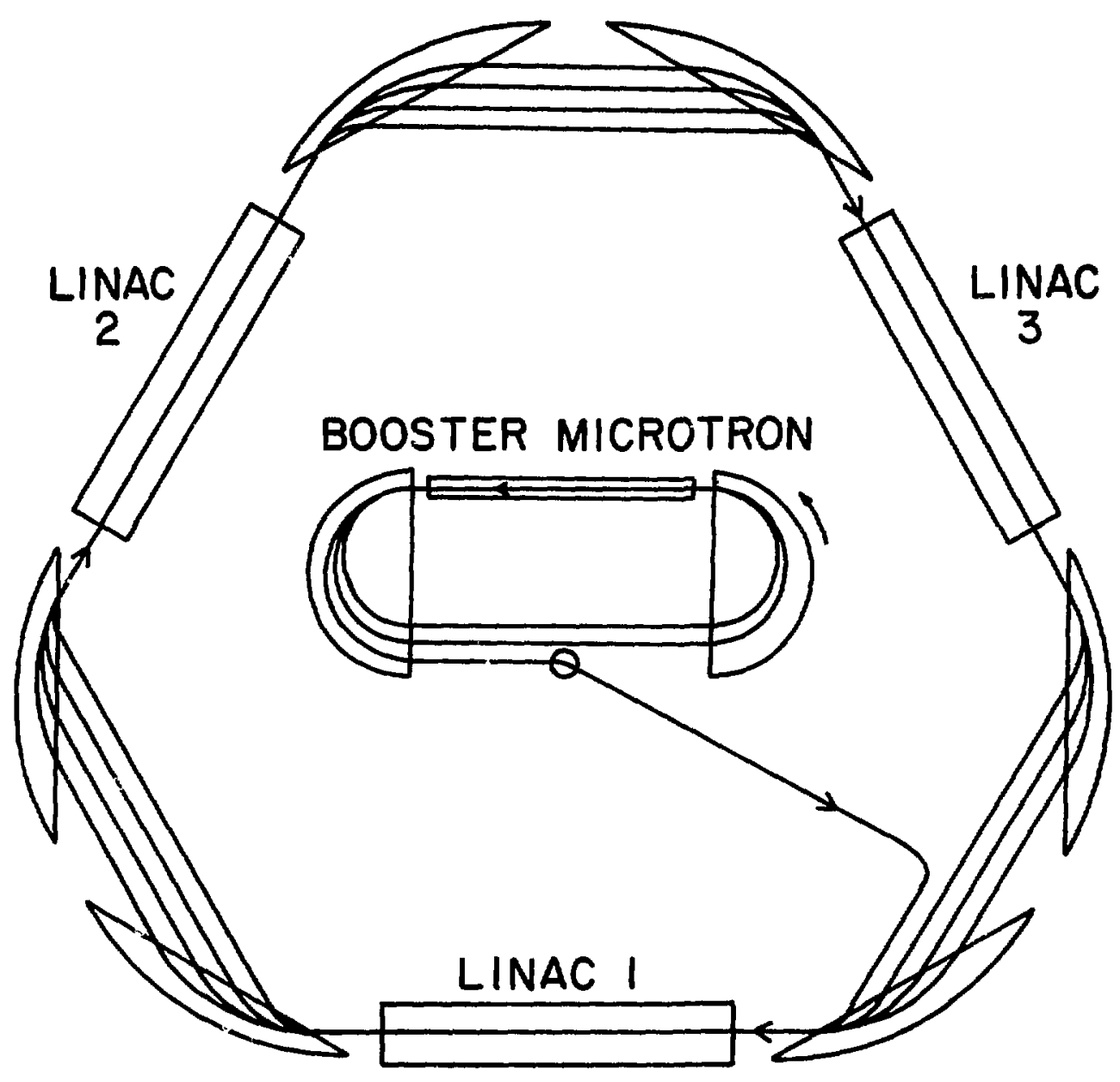

Fig. VIII-1. Schematic of the ANL 4-GeV C.W. hexagonal mictrotron, GEM. The accelerator is a six-sided microtron consisting of three dispersfve straight sections and three dispersion-free stralght sections where three linacs are located. A 185 race track booster microtron serves as an injector. Energy gain per turn is $105 \mathrm{MeV}$ and maximum current accelerated is 300 uamp. 
neutrons and the structure of nuclei. Colncidence measurements, made possible because of the $100 \%$ duty factor, will provide high selectivity and complete characterization of basic nuclear reactions which will make them a major component of the experimental program.

Argonne, through the GeV Electron Microtron (GEM) project is currently engaged in a vigorous effort to develop the technology for the electron accelerator; the purpose of the ANL program begun in 1980 has been to establish the technical merit of a multisided microtron as the most cost effective option for a national facility. Detailed conceptual designs for a multisided microtron have been developed. Simultaneously, members of the research staff have prepared preliminary conceptual designs for the spectrometers required in the experimental areas, and an architectural engineering firm has been engaged to complete a conceptual design of the buildings and services. A complete facility design with capital cost schedules will be available about November 1,1982 .

Much of the effort of the GEM Project is focused on an engineering and design study of the sector magnets which generate the magnetic guide fields in the multiside microtron configurations which would be employed for $\mathrm{GeV}$ accelerators. Unlike the race-track microtrons which can be used at lower energies $(5500 \mathrm{MeV})$, the optics of the multi-side microtron systems are characterized by strong vertical defocusing forces at orbit entry and exit from a magnet of typical design. To demonstrate scientific feasibility it is necessary to determine the central and fringe fields accurately and develop a system of additional focusing elements which will ensue orbit containment and high beam transmission. The initial phase of the ANL project has been devoted to a detalled design of a sector magnet for GEM and the construction of a high-precision computer controlled fleld measuring system for mapping fleld profiles of test units. This has been followed by the construction of a series of prototype magnets of the low-energy portion of the sector magnets corresponding to the flelds traversed in the first turns of beam operation where the beam optics are particularly difficult. While these tests are in progress a complete prototype will be constructed. Its design is expected to closely approximate that appropriate to a working accelerator design, but its primary purpnse is to serve as a benchmark for 3-D field calculations using recently developed computational techniques. Once the rellability of these calculations is established, they will be used to optimize the sector magnet design for the best transmitted beam quality. The prototype magnet will also serve as a test bench where systems of pole face windings designed to trin the field configurations and tune the individual orbit parameters can be studied. The fleld configurations of the final design will be used in orbit calculations and a beam breakup analysis and will be the basis for a complete facility design. 
a. Status of Microtron Sector Magnet Studies

Partial pole tip assemblies corresponding to several possible geometries for GEM have been fabricated and assembled with a return yoke once excited to design values. Measurements of field profiles with these partial prototypes with an initial precision of $0.3 \%$ have been completed. The data from these measurements Indicates the presence of unacceptably strong fringe flelds. Orbit analysis using RAYTRACE and TRANSPORT programs in interactive verslons has indicated that extensive empirical shimming would be necessary to achleve satisfactory fleld profiles for transmission of beams significantly below $100 \mathrm{MeV}$. These measurements were the basis for a decision to use a booster microtron system to provide an injection energy into the $\mathrm{GeV}$ microtron at sufficlently high energy to ensure good beam optics with contalnment systems of acceptable complexity. Data obtained show that yoke and coll to pole face geometry can be modifled to provide Improved fleld profiles. A new yoke and coll design has been completed and assembled. Field measurements using the new design are in progress.

Specifications have been established for a full prototype sector magnet and the engineering design has been completed. Component procurement is in progress. Fabrication will include a full size prototype pole piece which will be used to establish flatness and parallelism which Is feasible with current technology.

\section{b. Future Plans}

Because of postponement by D.O.E. of earliest consideration of construction proposals for a national electron facllity until January, 1983, design work for the microtron sector magnet has been scheduled for completion in late 1982. Fleld measurements on varlous models made with a precision of $\pm 0.1 \%$ and a spacial resolution of $0.5 \mathrm{~mm}$ w111 be completed during this period. Subsequent calculations using 3-D magnet programs such as TOSCA will be used to optimize the design. The results will be Incorporated Into a model of beam dynamics in the double-side microtron for establishing accelerator performance and the electron current threshold for 
beam breakup. The data w1ll furnlsh the base for evaluating alternative designs for a multisided microtron. At this time a decision will be made on a final magnet configuration which will be incorporated Into a design Including cost and time schedule. In this same perlod conceptual engineering design for beam transport, extraction, and vacuum systems will be performed. Analysis of beam dynamics in a hexatron accelerator will be carried out in order to develop a model of regenerative beam breakup. Prototype work will be carrled out on linac r.f. structures in order to determine those characteristics required to understand the beam breakup limit on circulating current. A construction proposal for a multi-GeV C.W. electron accelerator research facility including experimental areas will be submitted to D.O.E during late 1982 . 


\title{
ATOMIC AND MOLECULAR PHYSICS RESEARCH
}

\author{
INTRODUCTION
}

The Atomic Physics research in the Physics Division consists of six ongoing experimental programs as follows:

(1) Dissociation and Other Interactions of Energetic Molecular Ions in Solid and Gaseous Targets (D. S. Gemmell, E. P. Kanter)

(2) Electron Spectroscopy with Fast Atomic and Molecular-Ion Beams (D. Schneider)

(3) Beam-Foil Research and Collision Dynamics of Heavy Ions (H. G. Berry)

(4) Photoionization-Photoelectron Research (J. Berkowitz)

(5) High-Resolution, Laser-rf Spectroscopy with Atomic and Molecular Beams (W. J. Childs, L. S. Goodman)

(6) Theoretical Atomic Physics (K. T. Cheng) 


$$
\mid
$$




\title{
IX. DISSOCIATION AND OTHER INTERACTIONS OF ENERGETIC MOLECULAR IONS IN SOLID AND GASEOUS TARGETS
}

\author{
INTRODUCTION
}

Tightly collimated beams of molecular ions with energies variable in the range $0.5-4.5 \mathrm{MeV}$ are directed onto thin ( $100 \AA$ ) foil or gaseous targets. The distributions in energy and angle are measured with high resolution $\left(\sim 0.005^{\circ}\right.$ and $\left.\sim 300 \mathrm{eV}\right)$ for the resultant collisionally-induced dissociation fragments. The two major aims of the work are (a) a general study of the interactions of fast lons with matter, but with emphasis on those aspects unique to the use of molecular-ion projectiles and (b) a study of the structures of the incident molecular ions. These two different aspects of the work are mutually interdependent. In order to derive structure information about a given molecular ion, one needs to know details about the way the dissociation fragments collectively interact with the target in which the dissociation occurs. Similarly, a knowledge of the structure of the incident molecular clusters is important in understanding the physics of their interactions with the target. We have therefore begun our work with careful studies involving beams of the simplest and relatively well understood diatomic molecular ions $\left(\mathrm{H}_{2}^{+}\right.$, $\mathrm{HeH}^{+}$, etc.). Even with these, several new and interesting phenomena have been encountered (e.g., the interactions between the molecular constituents and the polarization oscillations that they induce in a solid target, the marked differences in dissociations induced in gases as compared with those in foils, the anomalously high transmission of some molecular ions through foils, and some striking electron capture phenomena when compared to atomic ions). As our understanding of these phenomena develops, we plan to go on to studies involving ever more complex projectile ions.

In the course of developing techniques for determining the stereochemical structures of molecular projectiles by coincident detection of dissociation fragments, 1 preliminary data have shown us the important problems which need to be addressed in order to obtain precise structural information. Most prominently, these problems include the need for a more detailed understanding of the physical processes involved in the interactions of these fast molecular ions with solid targets. In particular, this year we have tested varfous models of the "wake" forces experienced by heavy molecular-ion dissociation fragments by studying the modified stopping power of molecular clusters in solids.

\footnotetext{
${ }^{1}$ D. S. Germmell, Chem. Rev. $\underline{80}, 301$ (1980).
} 


\section{a. Molecular-Ion Stopping Power Measurements}

D. S. Gemmell, E. Johnson, E. P. Kanter, M. F. Steuer, and B. J. Zabransky

Following the discovery in 1974 of the influence of cluster effects upon the slowing down of ions in solids, there have been several experimental and theoretical investigations of this phenomenon. In general, the cluster stopping powers for 1 ight projectiles such as $\mathrm{H}_{2}^{+}$are fairly well described on the basis of wake models derived from Lindhard's dielectric function. The situation for heavy clusters, however, is more complex.

We have measured the stopping power ratio, of $\mathrm{N}^{+}$fragments from equal velocity beams of $\mathrm{N}^{+}$and $\mathrm{N}_{2}^{+}$. In contrast with the earlier work on light lons, we were able to select molecular $\mathrm{N}^{+}$fragment pairs that were "longitudinally" aligned to the beam direction upon exiting the foil. This orientational selectivity greatly simplifies the analysis.

The surprising results (see Fig. IX-1) show that contrary to the light-ion data, the stopping power of heavy molecular clusters is diminished from that of the corresponding monatomic projectiles. This implies that most of the induced negative polarization charge must lie between the separating fragment ions. None of the calculations performed to date, based on current linear wake models, is able to even qualitatively reproduce these ratios.

\section{b. Channeling of Molecular-Ion Beams}

D. S. Gemme11, E. P. Kanter, M. F. Steuer, and B. J. Zabransky

In another test of the various wake models that have been proposed, we have conducted a series of measurements in which Coulomb-exploding fragment ions are allowed to separate to distances comparable with the wake wavelength before exiting the target foil. This has not been possible previously because, for the thick foils necessary for such measurements, small-angle multiple scattering smears out the joint energy-angle distributions for the resultant fragments. By channeling a $\mathrm{HeH}^{+}$beam in a $700-\AA$ single crystal gold target, we were able to greatly refuce the multiple-scattering effects. High-resolution joint energy-angle distributions of the resulting proton dissociation fragments were measured for both axial and planar channeling orientations (see, e.g., Fig. IX-2). By varying the energy of the incident ion beam, we were able to observe both alignment and disalignment of molecular clusters. This was anticipated because of the 


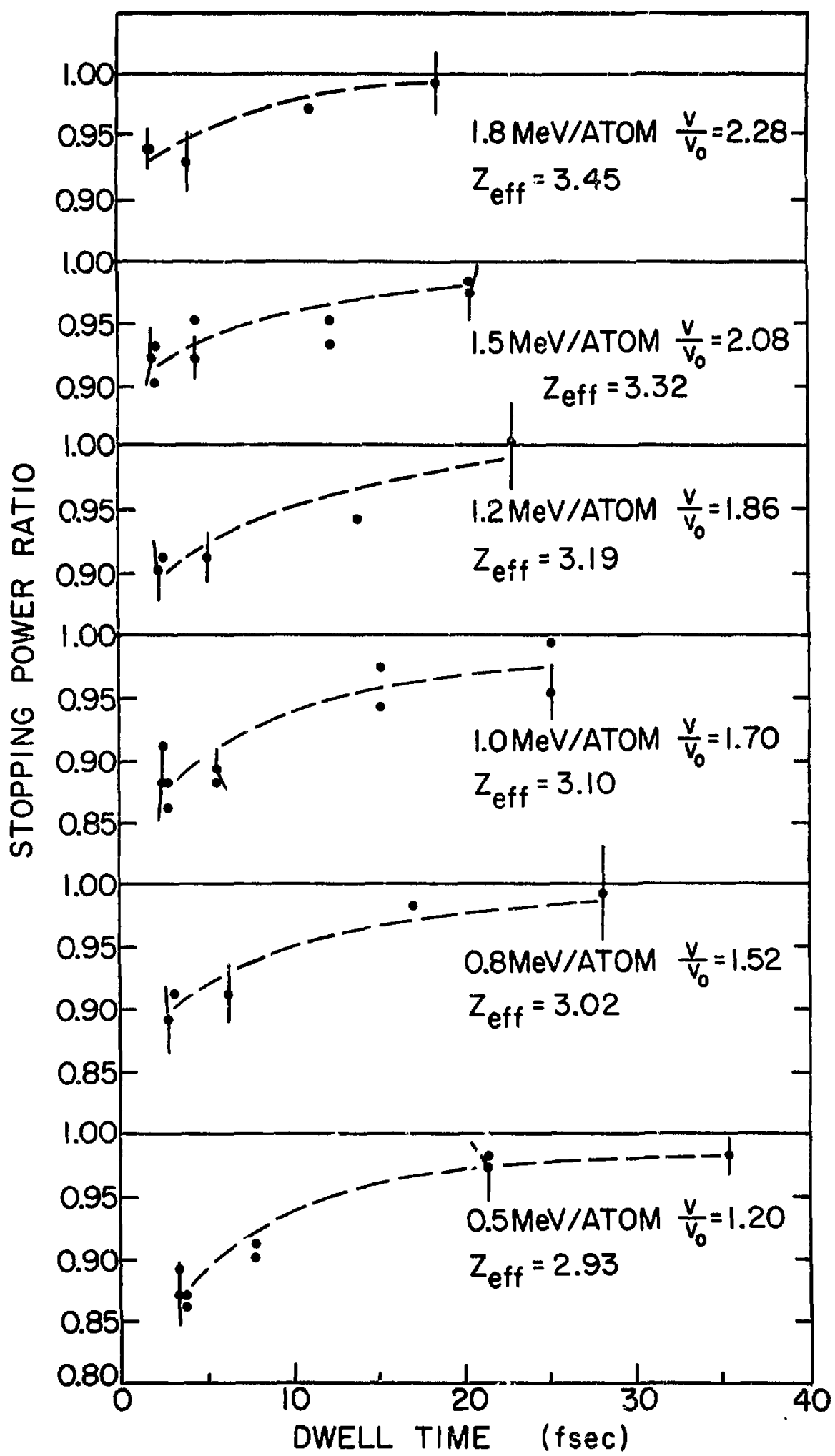

Fig. IX-1. Stopping power ratio for $\mathrm{N}_{2}^{+}$projectiles of various energies penetrating carbon foils. Only $\mathrm{N}^{+}$ fragments from "longitudinally" oriented fragment pairs are detected. 

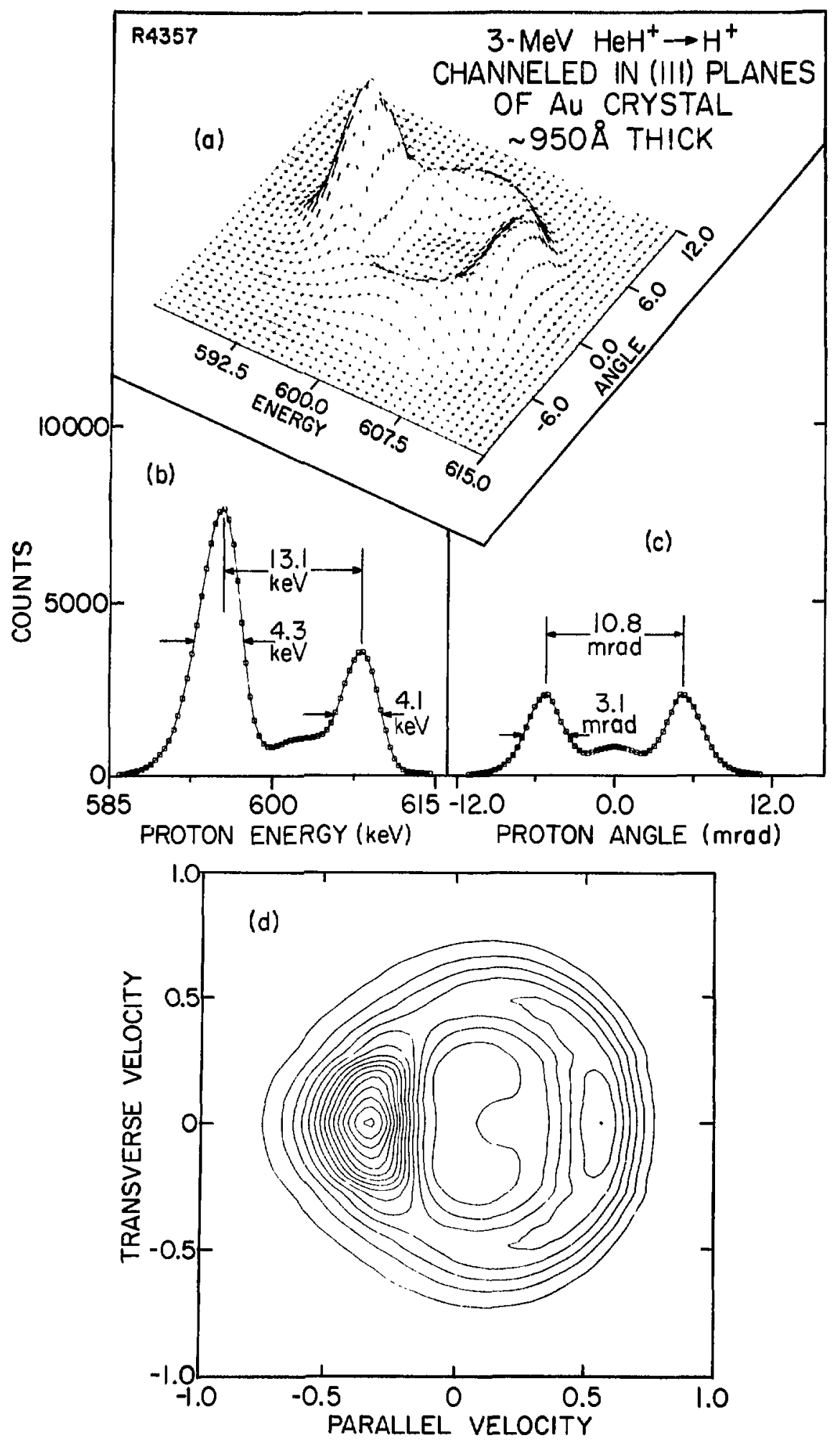

Fig. IX-2. Ring pattern for protons emerging in the (111) planar direction from a gold crystal $950 \AA$ thick bombarded by $3-\mathrm{MeV} \mathrm{HeH}$ incident along the same (I11) planar direction. In Fig. IX-2(d), the velocity scales are arbitrary, but equal. The angular coordinate in Figs. IX-2(a) and (c) Is measured with respect to the beam direction in the (111) plane. 
periodic nature of the wake potential in the solid, and should prove to be an Important quantitative test of the long-range behavior of the induced electron polarization charge trailing the ions.

\section{c. Computer Simulations of Foll-Induced Dissociations}

D. S. Gemmell, E. P. Kanter, I. Plesser, and Z. Vager

During the past year, we have explored the use of statistical mechanical techniques in the simulation of foil-induced molecular fragmentation experiments. While these techniques offer improved treatments of the effects of multiple scattering and molecular vibrations and rotations, several IImitations have been found. As was discovered in expensive Monte-Carlo calculations, the Gaussian form for the assumed multiple scattering distribution can be a severe drawback. In addition, due to computational difficulties, it has not been possible to use these techniques in calculations for target thicknesses in excess of $250 \AA$.

\section{d. Electron Spectroscopy of Fast Molecular Ions}

D. Schneider, D. S. Gemmell, E. P. Kanter, and B. J. Zabransky

We have measured and compared the line shapes and intensities for metastable Auger decays of carbon fragments from $\mathrm{C}^{+}$and $\mathrm{CO}^{+}$beams. We find, contrary to reports of another experimental grcup, that the decaying carbon atoms resulting from $\mathrm{CO}^{+}$dissociation are not strongly aligned with the beam direction. This new result js now in agreement with electron capture experiments with lighter molecular fons and with the foint energy-angle distributions obtained in the $\mathrm{CO}^{+}$fragmentation measurements. It is hoped that coincidence measurements of Auger decays with dissociation fragments will provide even greater sensitivity.

\section{e. Equipment Development}

D. S. Gemme11, E. P. Kanter, D. Schneider, M. F. Steuer, and B. J. Zabransky

Several projects were carrfed out during 1981 to improve existing experimental apparatus and to develop new equipment: 
(a) A two-axis crystal gonfometer was adapted for use on our beam 1ine. The adaptation added a third rotational axis and second translational degree of freedom for use in the molecular ion channeling experiments.

(b) The continued conversion to cryogenic pumps in our scattering chamber and flight tube sections of the beam line has improved our vacuum by an additional factor of two. This has reduced the problem of chargechanging collisions of heavy-ion fragments in the long flight path between the target and the electrostatic analyzer.

(c) A new target rack was constructed which allows the use of seven solid targets as well as a newly designed gas jet. This gas jet, and the increased vacuum pumping speed mentioned above, has allowed us to increase the gas target density in the beam by more than a factor of four over our old design. This was used to measure cluster stopping powers by comparing dissociation fragments which traversed solid targets with those which were separated in gas col1 istons.

(d) We have constructed two 45-degree parallel plate electron spectrometers. These spectrometers have a relative energy resolution of $2.6 \%$ and employing deceleration, can be improved to $0.03 \%$.

(e) The on-1ine PDP-11/45 has been connected, through the ANL Intra-Laboratory Network (ILN) to the central computer facility of the Laboratory. This high-speed link allows us to take advantage of the full computing power of the large central computers of the Laboratory while experiments are in progress, thus allowing complex diata analysis tasks to be performed on line. A number of new software tools have been developed for this purpose.

(f) A target stage capable of rotation by 360 degrees has been constructed for studies on the effects of different exit surfaces on molecular dissociation experiments.

\section{f. Contribution of Field-Ionized Rydberg Atoms to Convoy Electron Spectra}

D. S. Gemme11, Y.-Z. Gu, E. P. Kanter, D. Schneider, Z. Vager, and B. J. Zabransky

A prominent feature observed in the energy spectrum of electrons emitted in the forward direction from thin foils and gas targets under bombardment by fast ions is a sharp cusp-shaped peak occurring at an energy where the electron velocity matches the velocity of the emerging projectile 
ions. For fast light ions, these "cusp" electrons were believed to originate from the capture of target electrons into projectile-centered continuum states. Intense experimental and theoretical efforts have been directed towards understanding the measured cusps in terms of various theories of this so-called electron-capture-to-the-continuum (ECC) model, as well as a competing "wake-riding" model. To date, none of these various theoretical treatments has had any success in explaining the full range of experimental data now available from cusp electron measurements. In an effort to explain some puzzling features of molecular-ion disspciation experiments which had suggested the production of large numbers of high Rydberg atoms in fast beams, we recently tried to determine the extent to which the presence of significant numbers of such atoms could be affecting observations on cusp electrons.

If electron capture can occur into continuum states lying just above the ionization threshold of he projectile, there seems to be no a priori reason why capture into bound states lying just below the ionization threshold cannot also occur with comparable probability. The fate of Rydberg atoms emerging from a target can be expected to depend sensitively, and in ways difficult to predict, upon details of the experimental apparatus. Rydberg atoms have long radiative lifetimes, but they can be ionizad in quite modest electric fields. Certainly the electric fields used in most electron spectrometers suffice to ionize a large fraction of Rydberg atoms entering the spectrometer. Because it is customary in measurements on cusp electrons to pass the projectiles em rging from the target through a spectrometer, it is likely that field-ionizing Rydberg atoms will contribute electrons, traveling with the beam velocity, to the secondary electron spectrum.

In our experiment, ${ }^{1}$ we have incorporated a deflecting field intermediate between the target and electron spectrometer [Fig. IX-3(a)]. By jointly sweeping the voltages on the deflecting plates, and the electron spectrometer, we find that although electrons emerging from the target are eliminated as anticipated by the deflecting field, there exists a sizable contribution of beam-velocity electrons which originate after the deflecting

1Z. Vager, B. J. Zabransky, D. Schneider, E. P. Kanter, Y.-Z. Gu, and D. S. Gemmel1, Phys. Rev. Lett. 48, 592 (1982). 


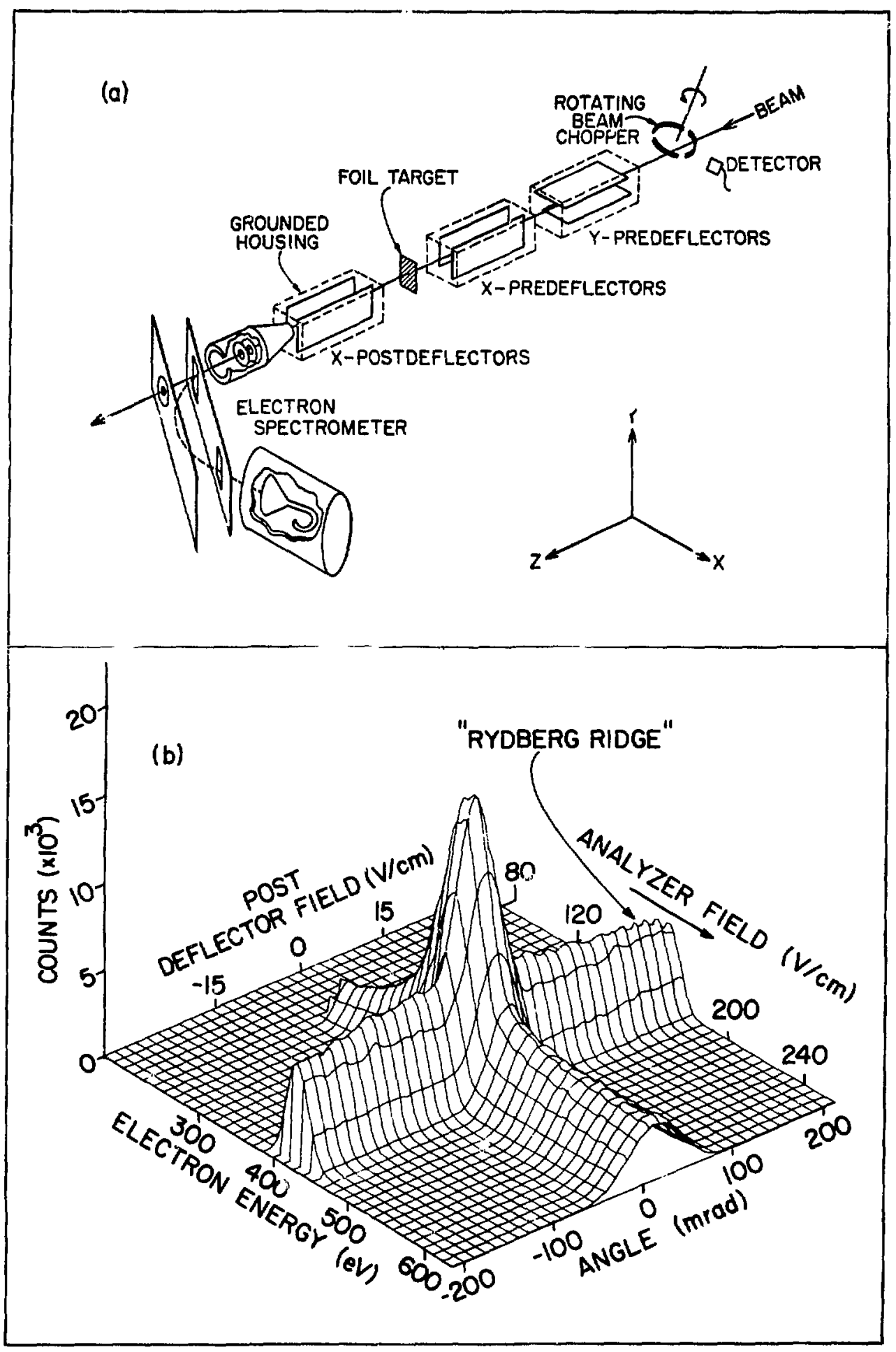

Fig. IX-3. (a) Schematic arrangement of the elements of the experimental setup within the target chamber. (b) Electron distribution, as a function of the post-deflector and spectrometer fields, measured for $3-\mathrm{MeV} \mathrm{He}^{+}$incident on a $2 \mu \mathrm{g} / \mathrm{cm}^{2}$ carbon foil. The energy scale applies to electrons from the target. The angle scale applies only to cusp electrons ( $400 \mathrm{eV}$ ) coming from the target. 


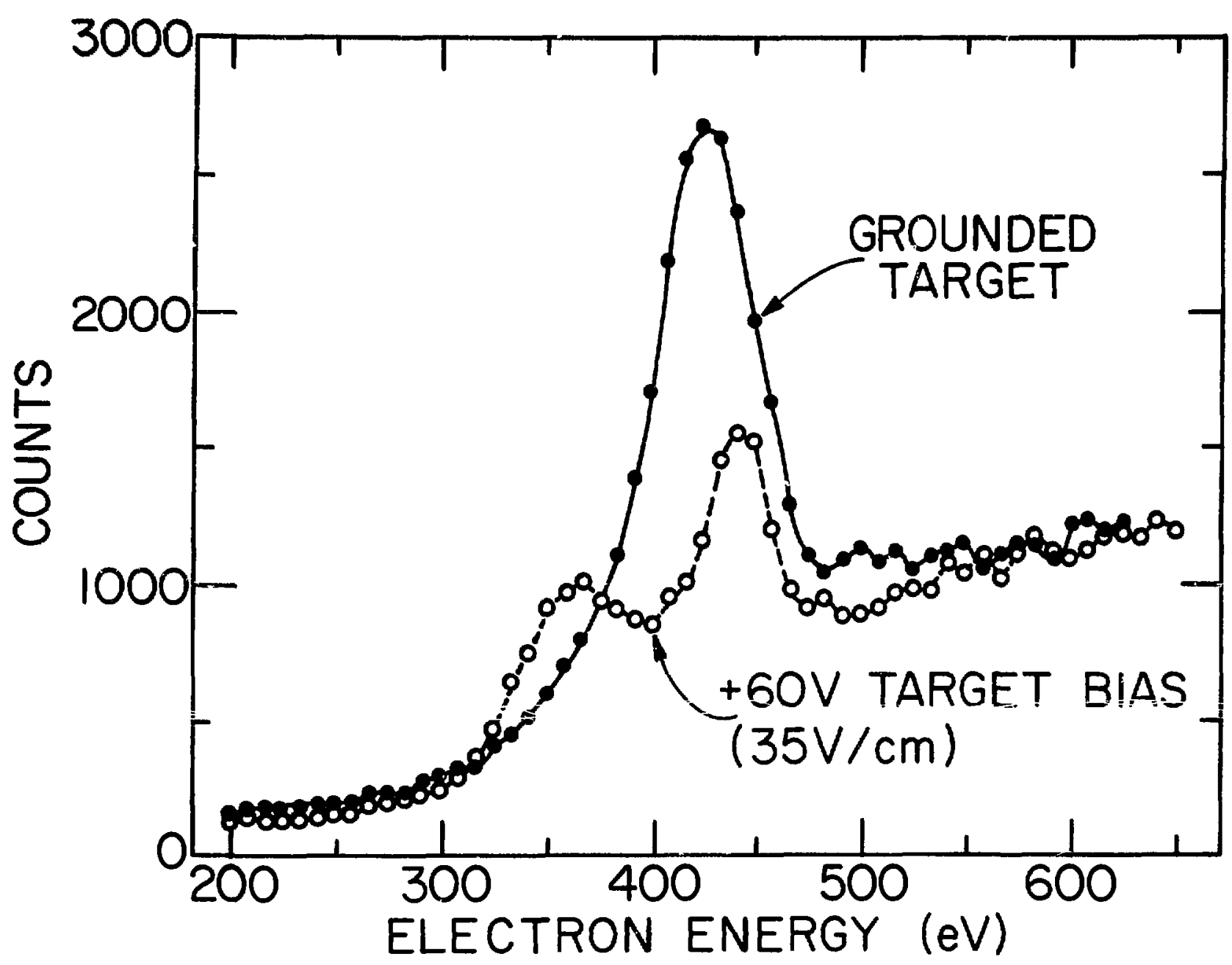

Fig. IX-4. Distributions of apparent energies for $0^{\circ}$ target electrons resulting from $750-\mathrm{keV}$ proton impact on a $2-\mu \mathrm{g} / \mathrm{cm}^{2}$ carbon foil. The solid curve was measured with the target grounded. The dashed curve resulted when a positive bias was applied to the target, lowering the energies of target electrons by $60 \mathrm{eV}$.

field [see Fig. IX-3(b)]. Further measurements (Fig. IX-4) with a +60 volt bias on the foil target shifted the cusp electrons to lower energy and allowed a separation of the target "cusp" from the fleld-ionizing Rydberg contribution.

These measurements, demonstrating the large yield of Rydberg atoms formed in excited fast ion beams, have far-reaching consequences in several areas of atomic collision physics. Most importantly, many measurements of charge-state yields and charge-changing cross sections must be reinvestigated in the light of this discovery. 


\title{
$X$. ELECTRON SPECTROSCOPY WITH FAST ATOMIC AND MOLECULAR - ION BEAMS
}

\author{
D. Schneider, D. S. Gemme1l, E. P. Kanter, and B. J. Zabransky
}

\author{
INTRODUCTION
}

The study of inner-shell ionization phenomena arising in energetic ion-atom collisions has been a field of experimental and theoretical interest for many years. The experimental techniques of high-resolution electron and $x$-ray spectroscopy coupled with the availability of intense well-collimated, highly-monoenergetic beams of a great variety of atomic and molecular ionic species at MeV energies, have opened many new avenues of research into inner-shell ionization phenomena. Although most of the early experiments were performed under single-collision conditions (dilute gas targets), it has recently been demonstrated that a wealth of valuable additional information can he obtained when solid targets (thin foils) are emiloyed. For studies involving low $-Z$ atoms $(Z \leq 20)$, the spectroscopy of Auger electrons is especially favored because of the low fluorescence yields.

The emission of Auger electrons following the dissociation of gasand foil-excited fast (MeV) molecular-ion beams is investigated by measuring single electron spectra with high resolution. The spectra from ionic fragments produced using molecular-ion beams are compared with spectra produced using : inatomic ion beams of the same velocity. The measurements include angular distributions (electron observation angle is varied) as well as the dependence on profectile energy.

In collisions with gases or solids, fast molecular ions rapidly lose their binding electrons. This leads to a dissociative molecular state that, in turn, gives rise to ionic fragments in various states of excitation. The fragments are repelled from one another by their mutual Coulomb forces and there ensues a somcalled "Coulomb explosion" of the molecular projectile. This causes an additional broadening of the lines in the observed Augerelectron spectra. The Coulomb-axplosion contribution to the line widths can be determined quantitatively by comparison with the spectral lines produced in measurements using the corresponding monatomic projectiles incident at the same velocity. Lines due to the decay of metastable states are particularly suited to this type of measurement since the lifetimes are long compared with the characteristic time for the Coulomb explosion $\left(210^{-15} \mathrm{sec}\right)$, thereby permitting nearly all of the initial Coulomb potential energy to be converted into the kinetic energy of the fragments before the Auger decay takes place. Recently some experiments along these lines were initlated by a group at the University of Frankfurt, who reported some evidence for surprisingly strong wake-alignment effects. 


\section{Auger Emission from Fragments Arising from Coulomb-Exploding Molecular-} Ion Beams

A series of measurements have been performed comparing the Augerelectron spectra (Fig. X-1) obtained with monatomic and diatomic projectile species that result in the formation of the metastable (1s $2 s 2 p)^{4} P^{0}$ state in lithium-like ionic fragments. The relative line intensity and lineshape for Auger emission from this state $i \mathrm{r}_{\mathrm{C}} \mathrm{C}^{3+}$ and $\mathrm{N}^{4+}$ using beams of $\mathrm{N}^{+}$and $\mathrm{N}_{2}^{+}$ and $\mathrm{C}^{+}$and $\mathrm{CO}^{+}$, etc. incident upon thin $(\sim 100 \AA)$ sarbon targets were measured as a function of observation angle (Fig. $\mathrm{X}-2)$. The measurements were performed at projectile velocities corresponding to about $100 \mathrm{keV} / \mathrm{A}$ beam energy.

In general the values obtained here for Coulomb broadening agree well with previously reported results, ${ }^{1,2}$ including those obtained from investigation of target molecules ${ }^{3}$ rather than of projectile molecules. However there is a significant difference in the angular dependence. Evidence for a $65 \%$ "anisotropy" in the line width which has been reported by the Frankfurt group ${ }^{2}$ is not found within the error limits of the present result. A slight "anisotropy" might be inferred from the comparison of line intensities. Even if an additional uncertainty due to the assumption of a Gaussian line shape is taken into account, no indication for strong "alignment" of the molecular clusters can be deduced. This result is consistent witi other investigations at Argonne on the dissociation of fast molecular ion beams in thin foils. 4 Measurements on the angular and energy distributions of fragment ions and of Doppler broadening produced in light emitted from the fragment ions after leaving the foil does not show evidence for strong "alignment" of molecules. 5

${ }^{1}$ J. Frischkorn, S. Schuhmann, R. Kluge, and K. O. Groeneveld, Physica Scripta 21, 63 (1980).

${ }^{2}$ K. 0. Groeneveld, Proceedings of NATO International Advanced Study Institute on "Molecular Ions," Kos, Greece, October 1980 (Plenum, New York), to be published.

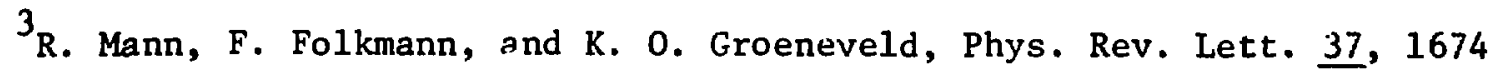
(1976).

${ }^{4}$ P. J. Cooney, D. S. Gemme11, E. P. Kanter, W. J. Pietsch, and B. J. Zabransky, Nucl. Instrum. Methods 170, 73 (1980). (1977).

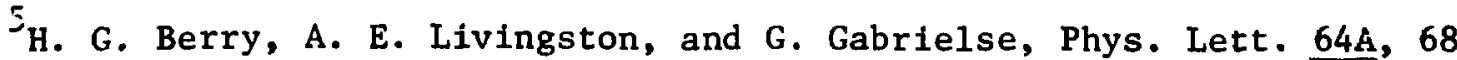




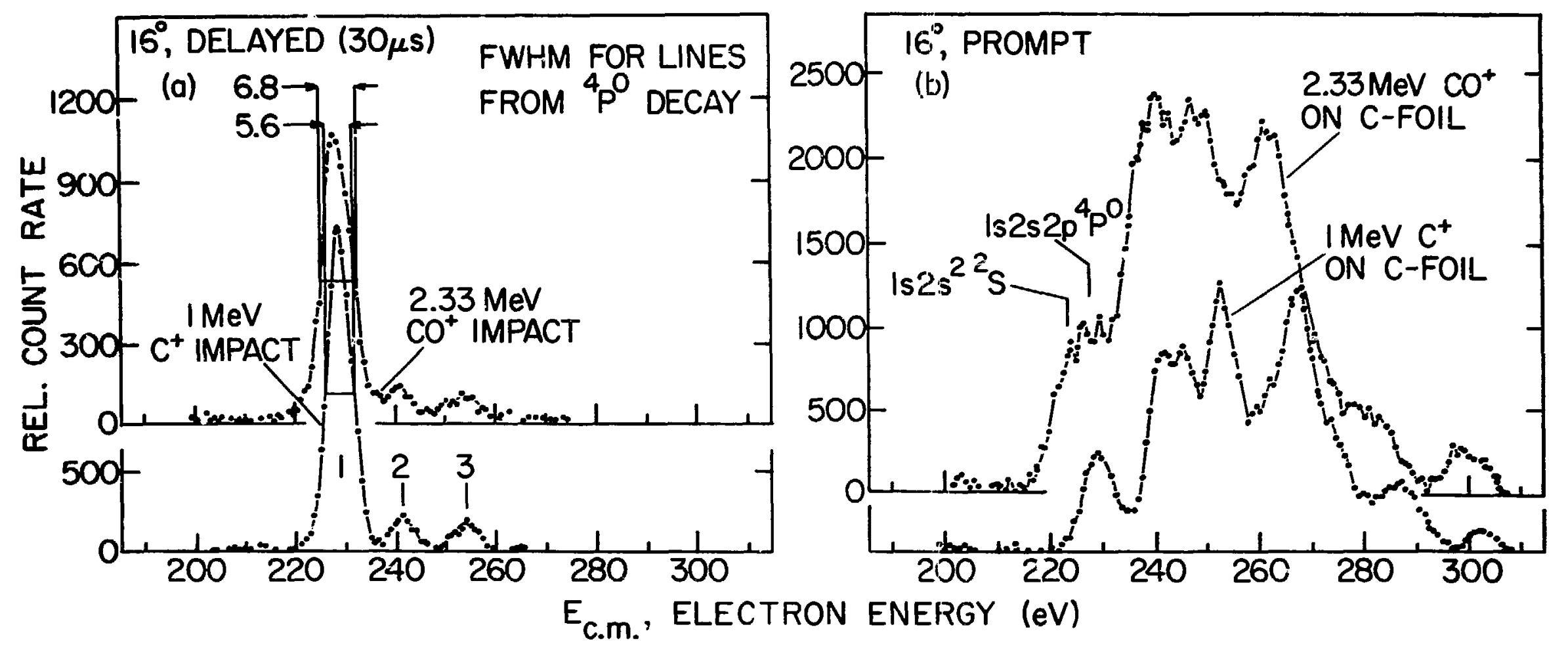

Fig. X-1. (a) Prompt electron spectra from $1-\mathrm{MeV} \mathrm{C}^{+}$and $2.33-\mathrm{MeV} \mathrm{CO}^{+}$ions excited in $5 \mu \mathrm{g} / \mathrm{cm}^{2} \mathrm{carbon}$ foils. A continuous electron background has been subtracted and the spectra have been transformed into the projectile rest frame. The laboratory observation angle $1: 16^{\circ}$ to the beam direction. Indicaced electron energies are in the projectile rest frame. (b) Time-delayed electron spectra produced in the coilision of $1-\mathrm{MeV} \mathrm{c}^{+}$and $2.33-\mathrm{MeV}\left(\mathrm{j}^{+}\right.$ions excited in thin carbon foils (time jelay $30 \mathrm{~ns}$ ). The experimental arrangement is the same as in Fig. X-1(b); however, the target was translated to an upstream position. The background has been subtracted and the spectra were transformed as in Fig. X-1(b) Peak 1 is due to the decay of the $4 \mathrm{p}^{0}$ decay; peaks 2 and 3 are assigned in $D$. Schneider, R. Bruch, W. Butscher, and W. H. E. Schwarz, Phys. Rev. A 24, 1223 (1981). 


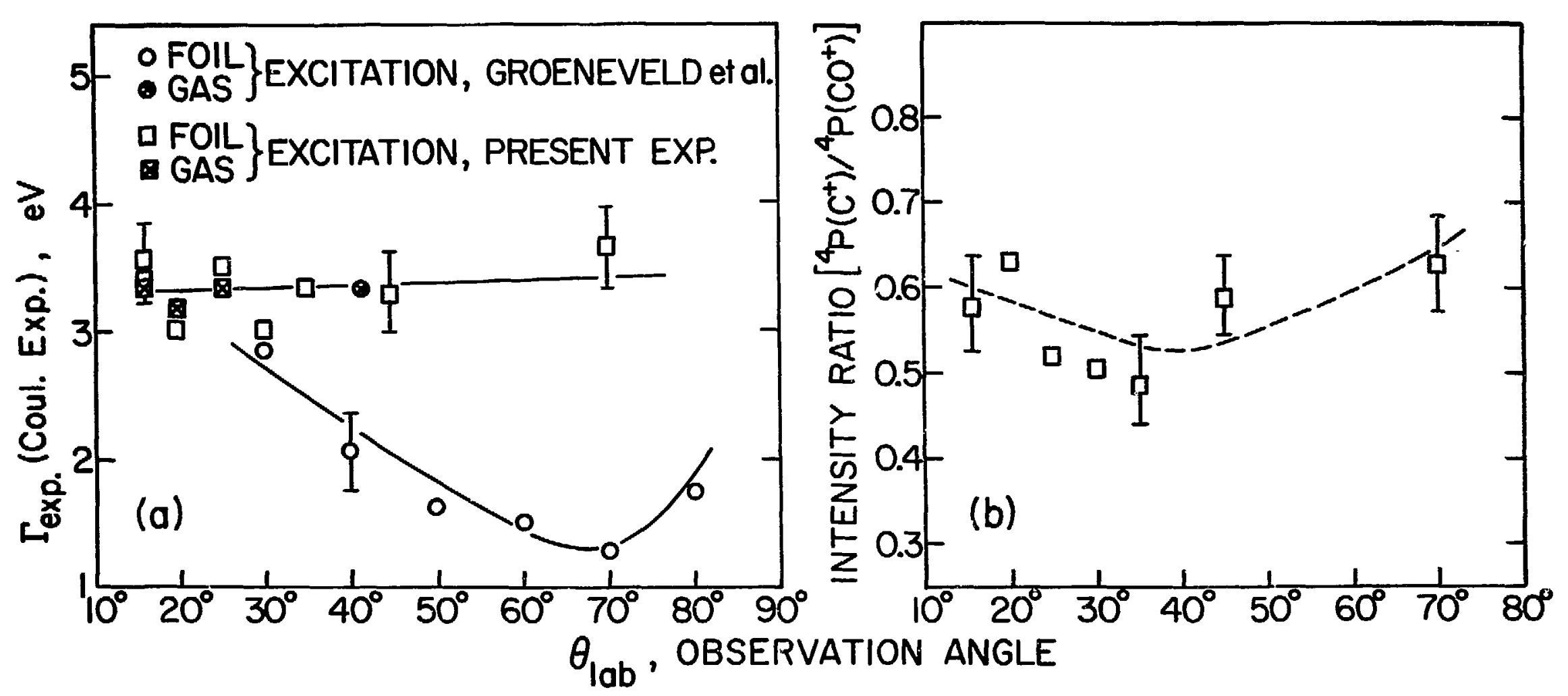

Fig. X-2. Experimental Coulomb-explosion broadening (a) and line-intensity ratios $\mathrm{Y}\left(\mathrm{C}^{+}\right) / \mathrm{Y}\left(\mathrm{CO}^{+}\right)(\mathrm{b})$ as a function of the angle of electron observation. The analyzed line is that arising from the decay of the metastable (1s2s2p)4 $\mathrm{p}^{0}$ initial state. $\mathrm{Y}\left(\mathrm{C}^{+}\right)$and $\mathrm{Y}\left(\mathrm{C \textrm {N } ^ { + }}\right)$ are the normalized yields in the corresponding lines obtained from the atomic and molecular case. 
It seems, however, from the presence of conflicting experimental results, that more sophisticated experiments should be performed in order to obtain information regarding, for example, the spatial orientation of clusters of molecular ion fragments emerging from solids. A state-dependent spatial aifinment could cause anisotropic electron emission. We propose to investigate this in further detail by performing coincidence measurements. Electrons from the jecay of a specific excited-fragment state will be measured in coincidence with the emitting fragment ion emerging at a given energy, angle, and charge state.

In addition to these spectroscopic investigations, total electron spectra following fast (MeV) atomic and molecular-ion impact have been measured with electron energies ranging from about $10 \mathrm{eV}$ to $1.0 \mathrm{keV}$. Yielsls of $\mathrm{K}$-shell Auger electrons from carbon and nitrogen in foil-excited molecular $\left(\mathrm{CO}^{+}, \mathrm{N}_{2}^{+}\right)$and atomic $\left(\mathrm{C}^{+}, \mathrm{N}^{+}\right)$ion beams have been measured and compared although no quantitative results are available at this point. 


\title{
XI. BEAM-FOIL RESEARCH AND COLLISION DYNAMICS OF HEAVY IONS
}

\author{
INTRODUCTION
}

Our present fast-ion-beam atomic physics program consists of three uajor parts, two of which are Investigations in atomic structure, and one of wh1ch Involves collision physics. Part (1) Involves work alnly at the Argonne Tandem accelerator $(30-100-\mathrm{MeV}$ Ion energy), and Parts (2) and (3) Involve the Argonne Dynamitron accelerator $(0.5-4.5-\mathrm{MeV}$ ion energy), and a low-energy test-bench factlity (0.02 - 0.15-MeV Ion energy).

(1) Atomic Structure of Highly Stripped Few-Electron Ions.

Th1s work provides tests of ab initio relativistic and quantum electrodynamic calculations (both by ourselves and others - see contribution of K. T. Cheng, Sec. XIV), We haye recently completed a set of precision measurements of the $1828^{3} \mathrm{~S}-182 \mathrm{p}{ }^{3} \mathrm{p}$ transitions of hellum-1ike $\mathrm{S1}, \mathrm{S}$, and $\mathrm{Cl}$, In collaboration with $\mathrm{A}$. E. Livingston at the University of Notre Dame. These results provide the f1rst tests of multi-electron contributions to QED theory. Our first calculations show good agreement. We are developing position-sensitive detector techniques to multiplex the observed spectra, thereby providing enchanced data collection rates, and we are making further technical Improvements in efficlency and IInearity.

Ab Initio calculations of transition energles in lons with 2 - 10 electrons are continuing, particularly in order to improve our estinates of relativistic and QED contributions.

(2) Atonic Structure of other Ions. Following our work in $\mathrm{LI}^{-}$, we have continued our searches for radiation in other negative lons. The search has 80 far been unsuccessful for $\mathrm{O}^{-}$and $\mathrm{H}^{-}$as well as a sintlar search for $\mathrm{H}_{2}{ }^{+}$line radiation.

The doubly excited spectra of 2,3 , and 4 electron atoms have been studied further in both the far and near ultraviolet spectral regions, especlaily in the low-z region (e.g., He, L1, and Ne).

Mean life measurements for some light lons have been wade in various tests of relativistic Hartree-Fock theory.

(3) Fo1l Interaction with Fast Ions. Work has continued in the atudy of optical polarization, both linear and c1rcular, of the light enitted from fast lons passing through thin tilted carbon folls. Observations on the energy and t1lt-angle dependence for triplet states in hellu are under study, and show some unexpected regularities. 
We have atudied the total yield and alignment of excited atonic states via obstrvations on the light yleld from molecular ion projectiles incident on thin folls. The molecular interactions within the solid and at the final surface of the foll can be studled in this way.

\section{a. Coherent Exc1tation of Hydrogen by a Thin Carbon Foll}

H. G. Berry, T. J. Gay, ${ }^{\text {and R. L. Brooks }}$

Continuing our observations of coherence of opposite parity states (e.g., 28-2p states) produced in thin foll excitation of hydrogen (thesis work of G. Gabrielse, 1979), we have observed its vartation for molecular-1on $\left(\mathrm{H}_{2}{ }^{+}\right.$and $\left.\mathrm{H}_{3}{ }^{+}\right)$1mpact. The coherence rapidly disappear when neighboring protons are within a few atomic diameters at the exit surface of the foll. These results help to set a distance estimate on the follhydrogen interaction. A detalled analysis shows that the $28-2 p$ coherence is affected by the long-range Coulomb field of the receding protons. Repulstve molecular states are formed at small internuclear distances near the foll surface. These states affect the $108 s$ of coherence. Some Irreproductbility in the resilts, however, has not yet been understood.

\footnotetext{
*Thes1s student, Univerafty of Chicago, Chicago, Illinols.
}

\section{b. Molecular Effects on Lyman- $\alpha$ Em1ssion in Beam-Fo1]. Spectroscopy}

R. L. Brooks and H. G. Berry

We have studied the total yield of Lyman- $\alpha$ (multi-exponential emission from the 2p state) from atomic hydrogen after dissociation of fast $\mathrm{H}_{2}{ }^{+}$and $\mathrm{H}_{3}{ }^{+}$Ions in thin carbon folls. All the observations show the same mult1-exponential decay of $\mathrm{H}^{0}(2 \mathrm{p})$ as a function of internuclear separation at the exit surface, suggesting two independent population mechanisms in the reconstitution region (FIg. XI-1). We have proposed a molecular breakup nodel which explains these results well, in addition to other recent measurements on molecular transmission and neutral hydrogen production. It shows good agreement with experiment over a dwell-tine range in the foll of 


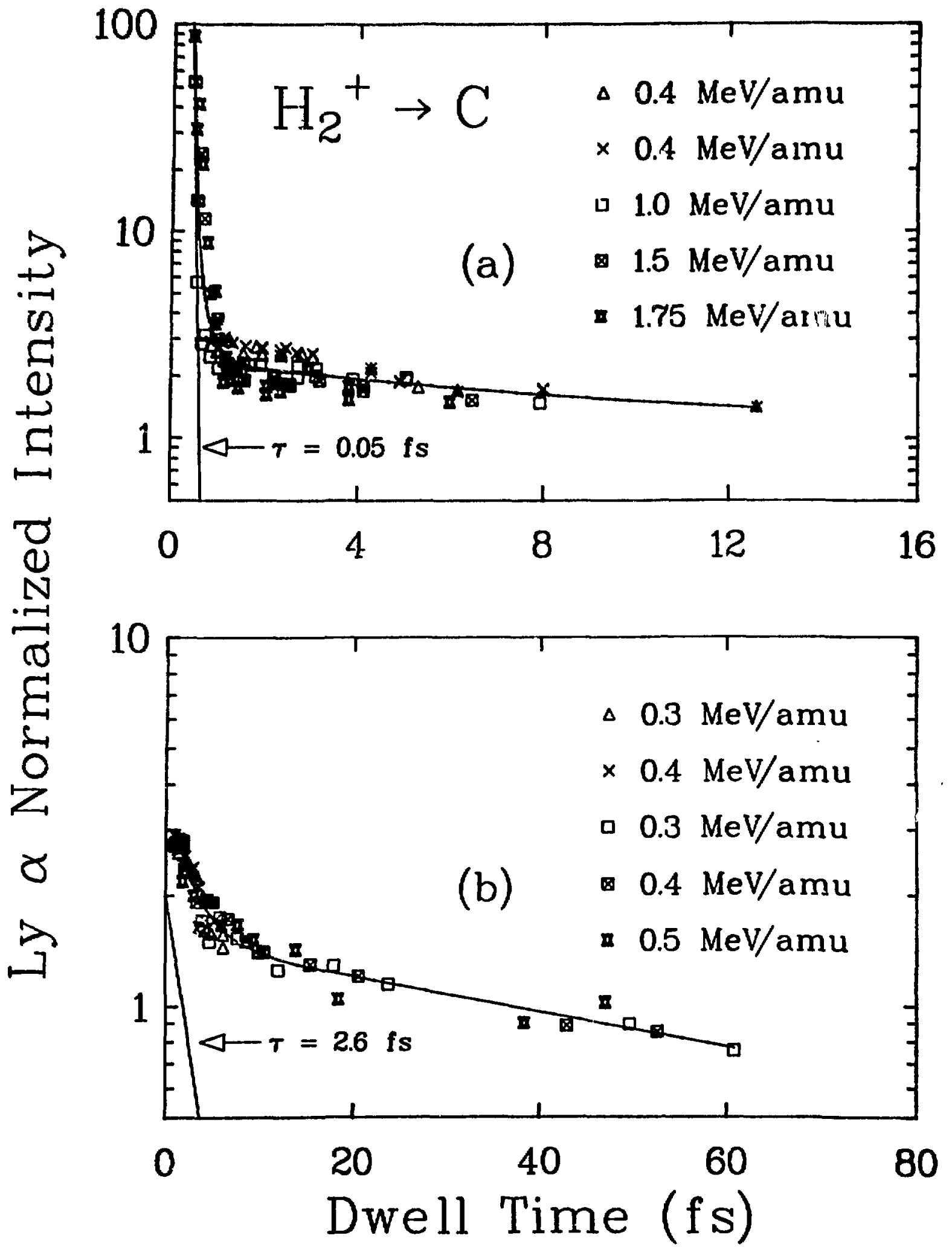

F1g. XI-1. Intensities of the atonlc hydrogen Lysan- $\alpha$ spectral line (1216 A), observed when molecular hydrogen lons $\left(\mathrm{H}_{2}^{+}\right)$pass through carbon target folls of varying thicknesses. The values are nornalized to the light yield of an equivalent bean current of atonic hydrogen (or protons). Short and long dwell-tine data are given separately, and are from different experiments. The lines represent three (a) and two (b) exponential fits to the data, wth the shortest lifetines labeled. 
0.2 to 50 femtoseconds. The application of the model to measurements of electron capture and loss cross section has been studied. 1

IR. I. Brooks and H. G. Berry, Phys. Rev. A 25, 161 (1982).

c. Orientation and Allgnment Parameters of Beam-Foll-Exc1ted HeI

H. G. Berry, R. L. Brooks, and E. H. Pinnington *

The alignment and orientation produced by the tilted-foll

excitation of He have been studied for a wide range of foll tilt angles and outgolng-atom velocities. In particular, two quantum states of different orbital angular momentum $\left(3 p^{1} P\right.$ and $\left.4 d^{1} D\right)$ were investigated and the results were compared with a number of previously proposed models. The results suggested contributions due to electron pickup and secondary electron as well as bulk effects. 1

Similar work on the $3 p^{3} \mathrm{P}$ state (at Argonne and the University of Alberta, Edmonton, Alberta) ${ }^{2}$ shows rather different values of the same parameters as measured for the $3 p^{1} P$ state. The results are almost Independent of beam energy between 100 and $1000 \mathrm{keV}$ (FIg. XI-2). This disagreement for states which differ only in spin is surprising, and may be due to detalls of the hellum energy level structure. We found also an unexpected conservation of symmetry in one of the allgnment parameters. Its independence of foll tilt may be a general feature of the fast-beam foll interaction.

\footnotetext{
*University of Alberta, Edmonton, Canada.

$1_{R}$. M. Schectman et al., Phys. Rev. A 22, 1591 (1980).

2 R. L. Brooks, H. G. Berry, snd E. H. Pinnington, Phys. Rev. A 25, 2545 (1982).
} 


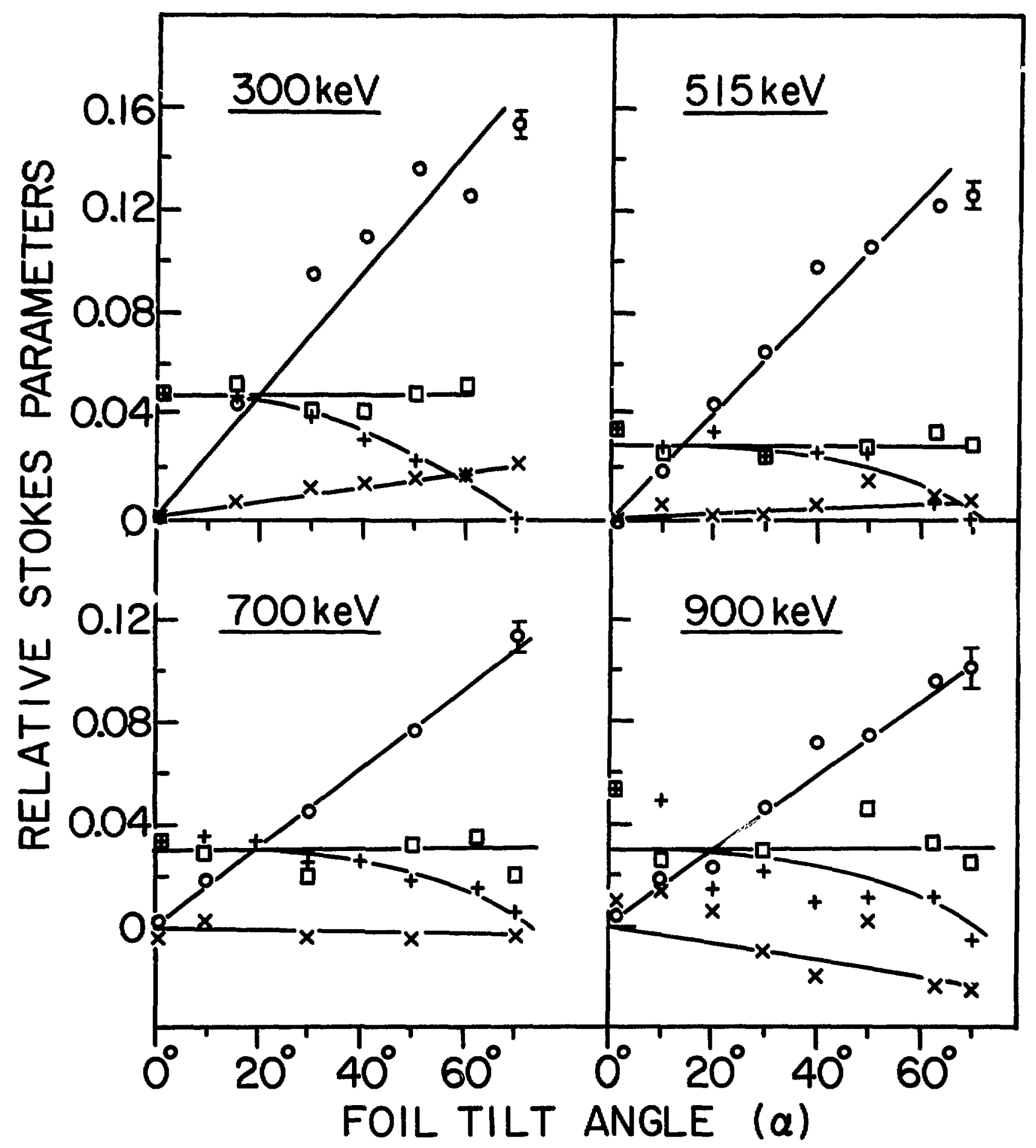

Fig. XI-2. The quantum beat averaged polarizations, $\langle S\rangle \mid\langle I\rangle(0),\langle C\rangle /\langle I\rangle(x)$, $\langle M>/<I\rangle(\phi=\pi / 2)(+),\langle M>/<I\rangle(\phi=0)(\square)$, for the $3889 \AA, 2 s^{3} s-3 p 3 P$ transition at beam energies of $300,515,700,900 \mathrm{keV}$. Errors for all points are Indicated by symbol of $S / I$ at $70^{\circ}$. 


\section{d. Quantum-Beat Studies of the ${ }^{3}$ He Hyperfine Structure}

V. F. Streif, R. L. Brooks, and H. G. Berry

The technique of beam-foll spectroscopy was used to study the hyperfine structure of ${ }^{3}$ He by the observation of zero-field quantum beats. ${ }^{1}$ Experimental results were obtained for the $2 p^{1} P-n d^{1} D$ transitions for $n=3$ to 8 , and preliminary results for $2 s^{3} s-3 p^{3} p$ and $2 p^{3} p-4 d^{3} p$. Theoretical predictions were made of the $\mathrm{nd}^{1} \mathrm{D}$ and $\mathrm{nd}^{3} \mathrm{D}$ hyperfine structure for $n=3$ to 10 . To within the experimental precision of $\pm 2-4 \mathrm{MHz}$, we find good agreement with our calculations. We expect to compare the observed variations in this simple system with recent laser-excitation measurements of hyperfine structures in highly excited levels in heavy complex atoms.

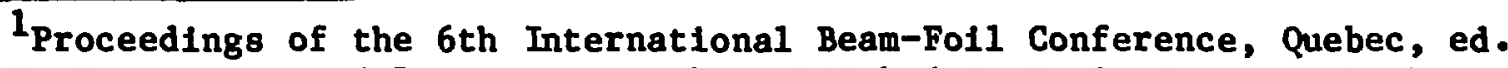
by E. Knystautas and R. Drouin, to be published in Nucl. Instrum. Methods.

e. Lamb Shift and Fine Structure of $n=2$ in Hellum-1ike Chlorine, Sulfur, and Silicon

R. DeSerio, ${ }^{*}$ H. G. Berry, R. L. Brooks, A. E. Livingston, ${ }^{\dagger}$ and S. Hinterlong ${ }^{\dagger}$

We have completed a set of precision wavelength measurements of the transitions $182 s{ }^{3} \mathrm{~S}_{1}-1 \mathrm{~s} 2 \mathrm{p}{ }^{3} \mathrm{P}_{0,2}$ of the helium-1ike lons of silicon, sulfur, and chlorine. ${ }^{1}$ We have calculated the wavelengths of these transitions for $Z=2-50$ using a nonrelativistic $1 / Z$ expansion, oneelectron Dirac energles, plus relativistic corrections in first-order perturbation theory, plus one-electron QED or Lamb-shift corrections. From comparisons of measurements of $z=4-26$ and theory, we find a discrepancy

\footnotetext{
*Thesis student, University of Chicago, Chicago, Illinois.

tUniversity of Notre Dame, Notre Dame, Indiana.

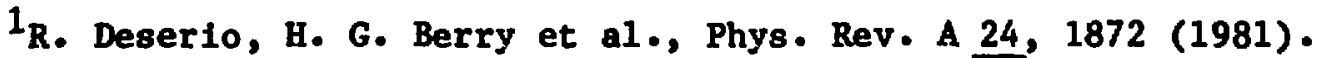


TABLE XI-I. ${ }^{3} \mathrm{P}_{2}$ energy matrix $\left(\right.$ in $\left.\mathrm{cm}^{-1}\right)$.

\begin{tabular}{|c|c|c|c|c|}
\hline & $\left(\alpha^{2} z^{2}\right)^{0}$ & $\left(a^{2} z^{2}\right)^{\prime}$ & $\left(a^{2} Z^{2}\right)^{2}+\cdots$ & Total \\
\hline & & $z=14$ & & \\
\hline \multirow{2}{*}{$\begin{array}{l}Z^{2} \\
Z^{1} \\
Z^{0}+\cdots \\
\text { Total } \\
\text { Mass polarization }=-\$ 6.92 \\
\text { Total energy }=-26266629.77\end{array}$} & $\begin{array}{r}-26885114.13 \\
693567.69 \\
-16293.15 \\
-26207839.59\end{array}$ & $\begin{array}{r}-59628.93 \\
1303.26 \\
-107.58 \\
-58433.25\end{array}$ & $\begin{array}{r}-299.39 \\
-0.62\end{array}$ & $\begin{array}{r}-26945042.45 \\
694870.33\end{array}$ \\
\hline & & $Z=16$ & & \\
\hline \multirow{2}{*}{$\begin{array}{l}Z^{2} \\
Z^{1} \\
Z^{0}+\cdots \\
\text { Total } \\
\text { Mass polarization }=-66.27 \\
\text { Total energy }=-34439599.87\end{array}$} & $\begin{array}{r}-35115337.19 \\
792650.73 \\
-16257.82 \\
-34338944.29\end{array}$ & $\begin{array}{r}-101724.57 \\
1945.39 \\
-140.52 \\
-99919.69\end{array}$ & $\begin{array}{r}-668.43 \\
-1.19\end{array}$ & $\begin{array}{r}-35217730.19 \\
794594.93\end{array}$ \\
\hline & & $Z=17$ & & \\
\hline $\begin{array}{l}Z^{2} \\
Z^{1} \\
Z^{0}+\cdots \\
\text { Total } \\
\text { Mass polarization }=-68.92 \\
\text { Total encrgy }=-38944532.14\end{array}$ & $\begin{array}{r}-39641981.95 \\
842192.64 \\
-16243.40 \\
-38816032.71\end{array}$ & $\begin{array}{r}-129640.96 \\
2333.43 \\
-158.64 \\
127466.17\end{array}$ & $\begin{array}{r}-962.73 \\
-1.61\end{array}$ & $\begin{array}{r}-39772585.64 \\
844524.46\end{array}$ \\
\hline
\end{tabular}

that is approximately $0.015 \mathrm{z}^{3} \mathrm{~cm}^{-1}$. We have shown that this arises from a first-order screening correction to the one-electron Lamb shift. Our calculations of the multi-electron QED corrections (e.g., Table XI-I) show excellent agreement with our measurements, which are an order of magnitide more precise than other meanurements in this range. 


\section{f. Position Sensitive Detector for UV Spectroscopy}

J. E. Hardis, * and H. G. Berry

We continue to investigate designs of orie-dimensional position sensitive detectors for the exit focus of our UV spectrometer. A Gallleo microchannel plate (MCP) serves as a front-end photon detector and signal amplifier. Techniques for imaging the output electron signal of the MCP are under study. Nonlinearities and drift in carbon-resistive-anode readouts limit the precision of the effective spectral dispersion curve obtained. Conductive wedges appear to hold promise, and computer modeling is underway. Applications of digital VLSI technology and multianode techniques are also under review.

\footnotetext{
*Thesis student, University of Chicago, Chicago, Illinols.
}

\section{g. Doubly-Exc1ted States In Lithlum}

H. G. Berry, R. L. Brooks, and J. E. Hardis*

The measured mean IIfe of the $182 \mathrm{p}^{2}{ }^{2} \mathrm{P}$ state of neutral 11thium has disagreed by more than a factor of two from theory for some time. A new measurement was needed for two particular reasons. First, this highly excited state, more than $50 \mathrm{eV}$ above the ground state, has been suggested as a good candidate as part of an $x$-ray laser; and secondly, a new deexcitation mechanism of simultaneous emission of a photon and electron (radiative auto-ionization-RA) has been suggested. Our new measurement of the radiative decay of $1 s^{2} 2 p{ }^{2} p^{0}-182 p^{2}{ }^{2} p$ at 207 A was midway between the two theoretical values including and excluding RA. ${ }^{1}$ Hence agreement has been attalned within $\pm 5 \%$.

\footnotetext{
*Thesis student, University of Chicago, Chicago, Illinois

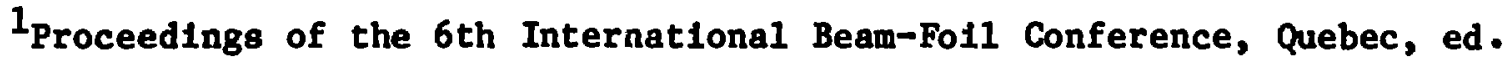
by E. Knystautas and R. Drouin, to be published In Nucl. Instrum. Methods.
} 
h. The Doubly-Excited Singlet States in Hellum

H. G. Berry, R. L. Brooks, and J. E. Hardis *

No optical emissions have previously been observed from doublyexcited singlet states in helium. This is principally because most such states decay rapidly through autoionization. There is stronger configuration mixing of the singlets than the triplets, and, in addition, all the $2 \ell 2 \ell^{\prime}\left(l, \ell^{\prime}=s, p\right)$ singlet states have very large natural width of several hundred millielectron volts. Thus, any transitions decayling to these states have large natural widths, of the order of $50 \AA$ at $3000 \AA$. We found two possible candidates for the $282 p{ }^{1} 0-2 p 3 p 1_{p}$ and $2 p^{2} 1_{D}-2 p 3 d$ ${ }^{1} D^{0}$ transitions at 4210 and $3298 \mathrm{~A}$, respectively. ${ }^{1}$ The former was Identified as a $\mathrm{CH}$ molecular band (foll sputtered) but the latter yields a linewidth for $2 \mathrm{p}^{3 \mathrm{~d}}{ }^{1} \mathrm{D}^{\circ}$ of $61 \neq 5 \mathrm{meV}$, in good agreement with theory. These are the largest linewidths observed in beam-foll spectroscopy.

\footnotetext{
*Thesis student, University of Chicago, Chicago, Illinois.
}

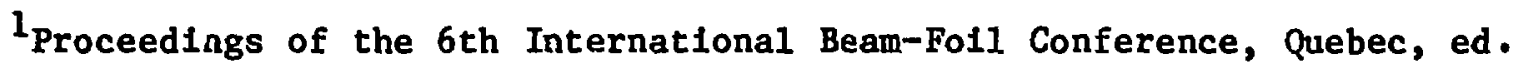
by $\mathrm{E}$. Knystautas and $\mathrm{R}$. Drouin, to be published in Nucl. Instrum. Methods.

1. Spectra of High Spin States in Light Elements

A. E. Livingston, ${ }^{*}$ H. G. Berry, K. T. Cheng, J. E. Hardis, ${ }^{\dagger}$

R. L. Brooks, and L. J. Curtis

Past experiments in this laboratory have identified the quartet ${ }^{1}$ $\left(1 s 2 s 2 p{ }^{4} p^{0}-1 s 2 p^{2}{ }^{4} p\right)$ and quintet ${ }^{2}\left(1 s 2 s 2 p^{2}{ }^{5} p-1 s 2 p^{3} 5 s\right)$ transitions in carbon, nitrogen, and oxygen. Higher beam energies at the Dynamitron, needed to excite the same doubly-exclted states in heavier elements, were not avallable. We have now a good source $(2-3 \mu \mathrm{A})$ of doubly-charged neon

\footnotetext{
*University of Notre Dame, Notre Dame, Indiana.

thesis student, University of Chicago, ChIcago, Illinois.

\#University of Toledo, Toledo, Ohio.

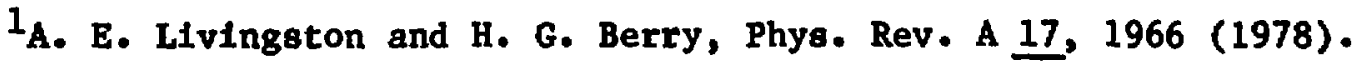

2H. G. Berry et al., Physica Scripta 25, 391 (1982).
} 


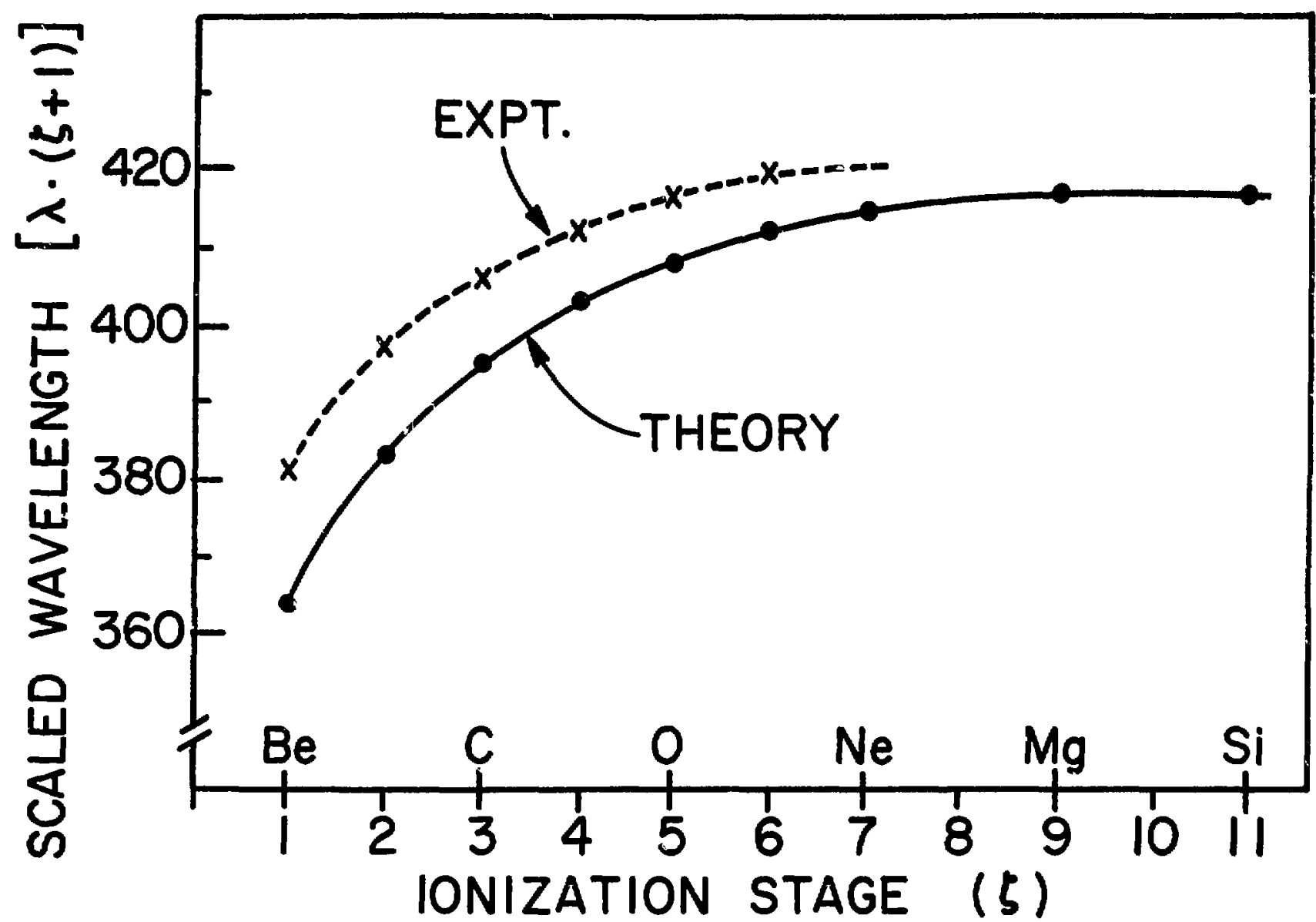

Fig. XI-3. Isoelectronic comparison of the scaled theoretical and experimental mean wavelengths of the $1 \mathrm{~s} 2 \mathrm{~s} 2 \mathrm{p}^{2} 5 \mathrm{p}-1 \mathrm{~s} 2 \mathrm{p}^{3} 5 \mathrm{~g} 0$ transitions. The experimental wavelength of $59.968 \mathrm{~nm}$ for F VI is an unpublished result of Martinson (Lund, Sweden, private communication). 
for the Dynamitron accelerator, which effectively doubles the terminal voltage for that element. The quartet spectral lines have been located, and measurements of their wavelengths and lifetimes are in progress. The search is continuing for the quintets. These measurements, and others at Notre Dame on magnesium, provide Increasingly good tests of relativistic atomic theory (which shows greater effects for heavier elements). Figure XI-3 compares theory and experiment.

j. Measurement of the Transition Probability of Singlet-Triplet Intercombination Lines in Neon

L. J. Curtis, * J. E. Hardis,,$^{\dagger}$ R. L. Brooks, and A. E. Livingston ${ }^{\dagger}$ Using the same doubly-charged neon beam mentioned above, we have begun to measure the Iifetimes of the neon $1 s^{2} 2 s 3 p{ }^{3} P_{0,1,2}$ states. The $j=1$ state has only about half the lifetime of the other two, and this difference is attributed to the extra decay channel to the $1 s^{2} 2 s^{2}{ }^{1} s_{0}$ state, accessible to it alone. Again, these measurements sxtend similar measurement of lighter elements done previously at lower energles (at Lund, Sweden). And again, this purely relativistic phenomenon is more pronounced in neon than in the lighter elements.

\footnotetext{
*University of Toledo, Toledo, Ohio.

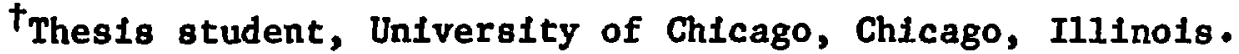

tUniversity of Notre Dame, Notre Dame, Indiana.
} 
k. Periodic Wavelength Dispergion in a Scanning UV Monochromator R. DeSerio, ${ }^{*}$ A. E. Livingaton, ${ }^{\dagger}$ and H. G. Berry

An analysis has been made of some wavelength dispersion problems assoctated with the McPherson Model 225 1-m vUV scanning monochromator. ${ }^{1}$ The measurements show a periodic error in the dispersion of such a monochromator, refocussed for a fast-ion beam 1ight source. They indicate a wavelength error from this source of up to $\pm 0.1 \mathrm{~A}$ with a period of $25 \mathrm{~A}$, this latter corresponding to one rotation of the grating drive screw. Knowledge of this mechanical error allows us, along with other users of this commercial device, to make corrections, vastly improving the utility of the device.

\footnotetext{
*Thesis student, University of Chicago, Chicago, Illinois.

tUniversity of Notre Dame, Notre Dame, Indiana.

$1_{R}$. DeSer10, Applied Optics 10, 1781 (1981).
} 


\section{PHOTOIONIZATION-PHOTOELECTRON RESEARCH}

\section{¿ITRODUCTION}

Our goal is to understand the electronic structures of atoms and molecules, and their ions, by observing the interaction of vacuum ultraviolet radiation with various specles and interpreting the dynamical results of this interaction. We have also embarked upon a direct study of molecular ion properties by studying the decomposition of these Ions Induced by ultraviolet laser 11ght. Our studies Involve fixed and variable wavelength photoelectron spectroscopy, photolonlzation mass spectrometry and laser-induced photodissociation spectroscopy.

This past year we have completed the photoelectron spectroscopic (PES) study of atomic tellurium. The intensity distribution of the fine structure Ion states is in good agreement with our previously developed irreducible tensor angular momentum coupling scheme. The remarkable success and generality of this model is as yet unmatched by even the most sophisticated ab initio theories for ionization of open-shell atoms. A series of PES experiments and calculations has been initiated on the rare earth trihalides, almed at elucldating the involvement of $4 \mathrm{f}$ electrons in chemical bonding.

Two photolonization mass spectrometric studies have been undertaken, and are essentially completed. The photodissoclative ionization of $\mathrm{NH}_{2} \mathrm{OH}$ was examined partly to determine the relative stabilities of $\mathrm{HNO}^{+}$and $\mathrm{NOH}^{+}$. The experiment established the heats of formation of both species quantitatively, and thereby enabled us to distinguish between two ab inftio calculations with opposite conclusions. A simflar study of thiomethanol and its deuterated forms $\left(\mathrm{CH}_{3} \mathrm{SH}, \mathrm{CH}_{3} \mathrm{SD}, \mathrm{CD}_{3} \mathrm{SH}\right)$ showed that $\mathrm{HCS}^{+}$was more stable than $\mathrm{CSH}^{+}$, but revealed an inadequacy in modern ab initio calculations involving second row atoms. Both the $\mathrm{HNO}^{+}$and $\mathrm{HCS}^{+}$results are significant in establishing reactions which can occur in interstellar clouds.

The experiment involving ultraviolet laser photodissoctation of molecular tons has passed two hurdles and is belng prepared for 1ts ultimate goal. 
Our photolonization research program is aimed at understanding the basic processes of Interaction of 1 ight with molecules, the electronic structures of molecules and molecular ions, and the reactions of molecular lons, both unimolecular and blmolecular. The processes and spectes we study are implicated in a wide range of chemical reactions, and are of special Importance in outer planetary atmospheres and in interstellar clouds. Our work also provides frultful tests for theorles of electronic structure, which help in the evaluation of widely applicable models for multi-electron systems. Most of this work is of a fundamental nature, but we also use the precise methods developed here to determine thermochemical quantities (heats of formation and lonization potentials) directly relevant in, e.g., reactions with ozone in the stratosphere, possible side reactions in a magnetohydrodynamic generator and reactions in interstellar clouds. Our experimental studies utflize five pleces of apparatus - two photoionization mass spectrometers and three photoelectron energy analyzers - each with spectal features.

(1) A three-meter normal-incidence vacuum-ultraviolet monochromator combined with a quadrupole mass spectrometer. This apparatus is capable of the highest resolution currently achieved in photolonization studies. It is also convenfent for Investigations of wavelength-dependent photoelectron spectra.

(2) A one-meter normal-incidence VUV monochromator mated with a magnetlc-sector mass spectrometer. This apparatus has higher mass resolution, is less discriminatory in relative lon-yleld measuremente, and can be used to study metastable lons. Higher intensity for weak signals can also be achieved.

(3) Two cylindrical-mirror photoelectron-energy enalyzers, wh1ch accept a large solid angle of photoelectrons, close to the "magic angle" of $54^{\circ} 44^{\prime}$. One has been extensively used for the determination of the photoelectron spectra of high-temperature species in molecular beams, and the other has on occasion been mated with the three-meter monochromator for studies of photoelectron spectra as a function of wavelength.

(4) A hemispherical electron-energy analyzer incorporated in a chamber which permits one to rotate the analyzer over a substantial fraction of $4 \pi$. This device is intended for angular-distribution measurements, and also enables us to study very-high-temperature species.

The experiment involving UV laser photodissociation of molecular Ions has progressed to the point where photofragment signals can be readily detected, without long searches. In the near future, the magnetic mass spectrometer $w f^{\prime}$ " be dedicated to this work.

One year ago, the interfacing of a multitask minicomputer with our experimental apparatus was described, and 1ts first test on the photolonization mass spectrometry experiment was about to be undertaken. Dur1ng the past year, a series of experiments on thloformaldehyde was successfully completed (see below) and hence this phase of interfacing is 
satisfactory. Programs have now been witten and tested which enable us to transfer data between our minicomputer and the main laboratory computer, thus permitting us to use the accumulated programs we had been using to treat the raw photolonization spectra. The minicomputer has also been used for calculations while accumulating experimental data.

Two of our other apparatuses still store data on 7-track magnetic tape. During the past year, the tape-reading device at the central computer has falled us on several occasions. We have been spared this difficulty with the minicomputer-interfaced apparatus; in the coming year, we plan to interface two (perhaps three) other experiments with the minicomputer .

The experiment Involving UV laser photodissociation of molecular lons successfully passed through two new stages. After preliminary feasibility experiments reported in 1981, the laser was focussed near the entrance slit of the mass analyzer, to improve momentum resolution and increase the number of detected events. During these tests, we noticed that stray scattered Ions, Inconsequential in most mass spectrometric experiments, were affecting ${ }_{5}$ our lon detector. Although these scattered lons represented $10^{-4}-10^{-5}$ of the primary beam, their counting rate was unacceptable. Tuning and allgnment changes had 11ttle effect. The problem has been solved by electronically pulsing the lon beam at the laser repetition frequency, but with an "on-time" substantlally larger than the laser pulse. In this way, the number of laser-generated events remains unchanged, but the scattered Ion background has been reduced by three orders of magnitude.

Other technical progress Included:

(1) The completion of a highly satisfactory photoelectron experiment on atomic tellurium at temperatures up to $1700^{\circ} \mathrm{K}$, with experimental intensities in good agreement with our irreducible tensor analysis (IRTA) calculations.

(2) The Inftiation of a photoelecton spectoscoplc study of rare earth trihalides, to examine the role of $4 f$ electrons in chemical bonding.

(3) The completion of a photolonization mass spectrometric study of hydroxylamine, $\mathrm{NH}_{2} \mathrm{OH}$, afmed at determining the relative stabilities of HNO ${ }^{+}$and $\mathrm{NOH}^{+}$.

(4) The completion of a photolonization mass spectrometric study of thiomethanol and its 18otopes $\left(\mathrm{CH}_{3} \mathrm{SH}_{2} \mathrm{CD}_{3} \mathrm{SH}, \mathrm{CH}_{3} \mathrm{SD}\right)$ partially directed at the relative etabilities of $\mathrm{HCS}^{+}$and $\mathrm{CSH}^{+}$.

These experiments are datalled below. 
a. Photoelectron Spectra of Open-She11 Atoms, w1th Emphasis on Tellurium

G. L. Goodman and J. Berkowitz

We had previously developed a model involving intermediate coupling and Irreducible tensor analysis to explain the changing relative intensities of the fine structure states $\left({ }^{3} \mathrm{P}_{0,1,2} ;{ }^{1} \mathrm{D}_{2} ;{ }^{1} \mathrm{~s}_{0}\right)$ in the photoelectron spectra of the halogen atoms $\mathrm{Cl}, \mathrm{Br}$ and $\mathrm{I}$. This "geometric" model was astonishingly successful in reproducing the experimental data. It was desirable to know whether the halogens represented a fortuitous clrcumstance, or that the model had more general applicabllity to other coupling schemes. Atomic tellurium, with a large spin-orbit interaction and a coupling scheme which produces the states ${ }^{4} \mathrm{~S}_{3 / 2} ;{ }^{2} \mathrm{D}_{3 / 2,5 / 2} ;{ }^{2} \mathrm{P}_{1 / 2,3 / 2}$ in the photoelectron apectrum was chosen as the test case. Solid tellurfum normally generstes the dlatomic molecule $\mathrm{Te}_{2}$ upon vaporization. We found that using solid $\mathrm{Cu}_{2} \mathrm{Te}$ as a sample, together with superheating of the vapor, produced predominantly atomic tellurium. This experiment necessitated operation at the highest temperature we have yet attempted in photoelectron spectroscopy - up to $1700^{\circ} \mathrm{K}$. A very satisfactory spectrum was obtained, which was in good agreement with our predictions based on the IRTA model (see Fig. XII-1). These results indicated a general applicabllity of the model. A paper describing these results, together with predictions for other open-shell atoms, is being written.

b. Photoelectron Spectra of Lanthanide Trihalides

B. M. Ruscić, G. L. Goodman, and J. Berkowitz

Modern molecular ab initio quantum mechanical calculations are currently able to produce quite reasonable molecular properties (e.g., geometry, orbital energles, vibrational frequencies, dissociation energies) for polyatomic systems that are not too large, and that contain elements not heavier thin those in the first row of the perlodic chart. Molecules containing second row elements have been calculated with poorer accuracy, and those containing transition, rare earth or actinide elements probably require some pseudopotential simplifications. Hence, some basic questions regarding molecular bonding currently require other techniques to find answers. Some time ago, we obtalned the photoelectron spectra of several 


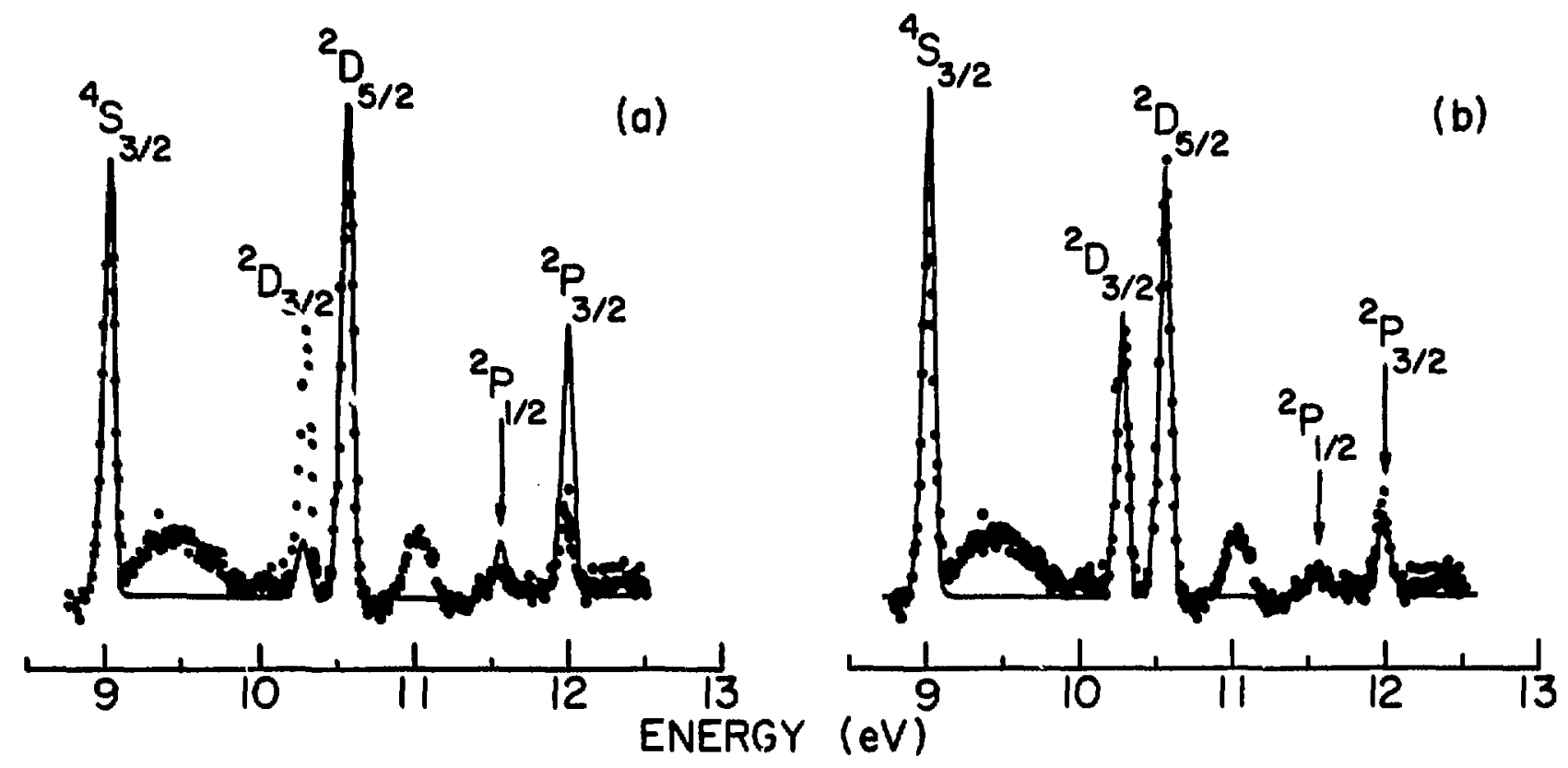

Fig. XII-1. Photoionization of atomic tellurium at $1400^{\circ} \mathrm{C}$. The 5 sharp peaks correspond to 5 states of the ion $\mathrm{Te}^{+}$formed in the process. The additional broad bands are due to $\mathrm{Te}_{2}{ }^{+}$, an impurity. In panel (a), a conventional calculation ( $r$ ferred to as the L-S coupling 1 imit in physics) is used to estimate the relative intensities. In panel (b), our recently described method (irreducible tensor analysis or IRTA) is used to estimate the relative peak intensities, and is seen to match the experimental reaults very we11. This agreement between experiment and calculation represents a major advance in our understanding of the photolonization process in certain difficult atoms (open shell atoms).

transition metal dichlorides and dibromides. With the ald of multiple scattering $X_{\alpha}$ calculations, we were able to show that the metal $3 d$ orbitals were the most loosely bound electrons in early members of the transition serles ( $\mathrm{kn}, \mathrm{Fe}$ ) and then were drawn in as one proceeded to higher nuclear charge, so that these $3 \mathrm{~d}$ orbitals became core orbitals when the $3 \mathrm{~d}$ shell was filled $(\mathrm{Zn})$. Hence, the molecular bonding in early members of the transition serles should be strongly influenced by $3 d$ orbitals.

Conventional wisdom until recently was that the 4 forbitals in the lanthanide molecules were too deep to be involved in molecular bonding, but the $5 f$ orbitals in actinide molecules could have some influence. In order to test the former hypothesis, we have initlated a photoelectron 
spectroscoplc study of the lanthanide trihalides, and supplemented this experimental work with relativistic $X \alpha$ calculations superior to those available for the transition metal compounds. These experiments require temperatures $\sim 1000^{\circ} \mathrm{C}$. The technology for such experiments has been demonstrated in only two or three laboratorles in the world. Furthermore, in order to distinguish $4 \mathrm{f}-11 \mathrm{ke}$ orbitals from ligand orbitals, it is necessary to perform such studies with at least two incident photon energles (usually 21.2 and $40.8 \mathrm{eV}$ ). For several reasuns, PES at $40.8 \mathrm{eV}$ is considerably more difficult. We have succeeded in obtaining photoelectron spectra of several trihalides (chlorides, bromides and lodides) of representative lanthanide metals at $21.2 \mathrm{eV}$ with good resolution. We are currently supplementing these data with some results at $40.8 \mathrm{eV}$. The experimental results are helping to refine the calculations. The overall plcture is becoming clear, and it seems to be at odds with the conventional one.

c. Photolonization Mass Spectrometry of $\mathrm{NH}_{2} \mathrm{OH}$ : Heats of Formation of $\mathrm{HNO}^{+}$and $\mathrm{NOH}^{+}$

R. E. Kutina, G. L. Goodman, and J. Berkowitz

Wavelength-dependent mass spectra have been obtained for the most prominent ions produced upon photolonization of hydroxylamine. Two distinct thresholds are observed for mass 31 (see Fig. XII-2), which we attribute to onsets for formation of $\mathrm{HNO}^{+}$and $\mathrm{NOH}^{+}$. The corresponding heats of formation are $\Delta \mathrm{H}_{\mathrm{f}, 0}^{\circ}\left(\mathrm{HNO}^{+}\right)=256.8 \pm 1.4 \mathrm{kcal} / \mathrm{mole}$ and $\Delta_{\mathrm{f}, 0}^{\circ}\left(\mathrm{NOH}^{+}\right)$ $=274.8 \pm 0.7 \mathrm{kcal} / \mathrm{mole}$, in excellent agreement with recent ab initio $\mathrm{CI}$ calculations. The threshold for mass 32 , which we tentatively ascribe to $\mathrm{H}_{2} \mathrm{NO}^{+}$, corresponds to $224.6 \pm 0.2 \mathrm{kcal} /$ mole for 1 ts heat of formation. Each of these entities can therefore be produced in an exothermic reaction of $\mathrm{H}_{3}{ }^{+}$with $\mathrm{NO}$, and represent mechanisms for depleting $\mathrm{NO}^{-}$in the interstellar clouds. Experimental breakdown diagrams are generated and compared with predictions of QET. It is found necessary to invoke a 3-body decomposition to explain some observations at higher energy. A theory is presented to accommodate this process, within the framework of QET. This work has been accepted for publication in J. Chem. Phys. 


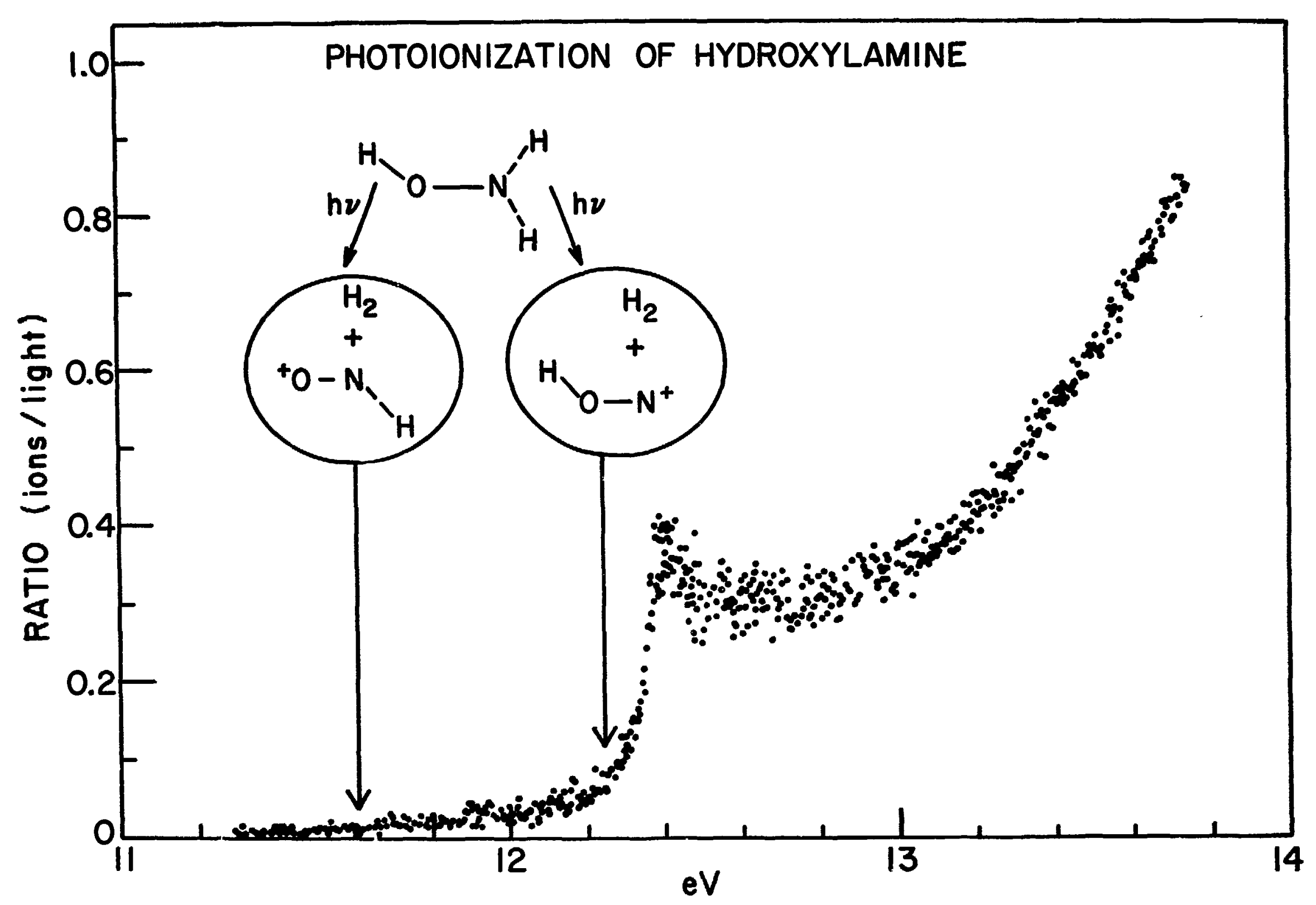

总

Fig. XII-2. Threshold region of $\mathrm{M} 31$ in the photoionization of $\mathrm{NH}_{2} \mathrm{OH}$. Two onsets are observed, corresponding to the energetically favored HNO+ product, and the sterically favored NOH product. 
d. Photoionization Mass Spectrometric Study of $\mathrm{CH}_{3} \mathrm{SH}_{2} \mathrm{CD}_{3} \mathrm{SH}_{4}$ and $\mathrm{CH}_{3} \mathrm{SD}$.

R. E. KutIna, A. K. Edwards, G. L. Goodman, and J. Berkcwitzz

Earlier electron impact studies showed that $\mathrm{CD}_{3} \mathrm{~S}^{+}$had a slightly lower appearance potential from $\mathrm{CD}_{3} \mathrm{SH}$ than did $\mathrm{CD}_{2} \mathrm{SH}^{+}$. We have verified this observation by photolonization (FIg. XII-3), but find that $\mathrm{CH}_{2} \mathrm{SD}^{+}$ $\left(\mathrm{CH}_{3} \mathrm{SD}\right.$ ) has a lower appearance potential than $\mathrm{CH}_{3} \mathrm{~S}^{+}\left(\mathrm{CH}_{3} \mathrm{SD}\right.$ ) (FIg. XII-4). The primary reason for this reversal is a thermochemical isotope effect, but the mechanism of formation, and the structure of the fragment ion, are st11l subject to controversy. The 1sotopic studies reveal that $\mathrm{HCS}^{+} 1 \mathrm{~s}$ more stable than $\mathrm{CSH}^{+}$, In agreement with a recent ab initio $\mathrm{CI}$ calculation. However, the absolute $\Delta H_{f, 0}^{\circ}\left(\right.$ HCS $\left.^{+}\right)$obtained by us differs significantly from the corresponding value obtained from the calculation, although our value is in excellent agreement with one based on relative proton affinity measurements. This observation corroborates the view that $\mathrm{HCS}^{+}$should be observable in the interstellar dust clouds. The fragmentation behavior of $\mathrm{CH}_{3} \mathrm{SH}^{+}$is markedly different from $\mathrm{CH}_{3} \mathrm{OH}^{+}$, including a much greater tendency for H/D 1sotopic scrambling. This work Is currently being prepared for publication. 


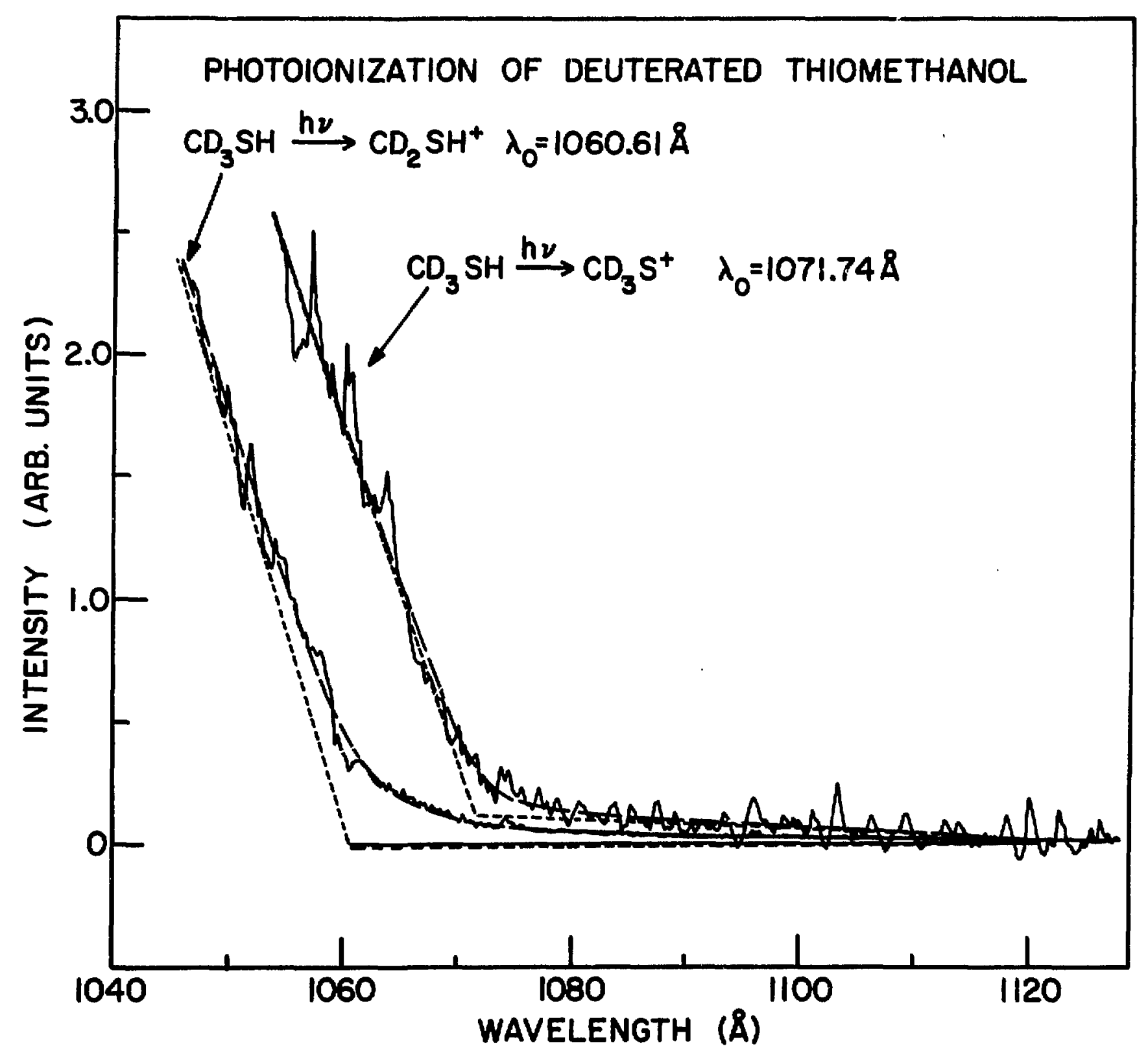

Fig. XII-3. Threshold regions for $M 50, \mathrm{CD}_{3} \mathrm{~s}^{+}$, and $\mathrm{M} 49, \mathrm{CD}_{2} \mathrm{SH}^{+}$from the photoionization of $\mathrm{CD}_{3} \mathrm{SH}$. The $\mathrm{CD}_{3} \mathrm{~S}^{t}$ is seen to have the lower threshold. 


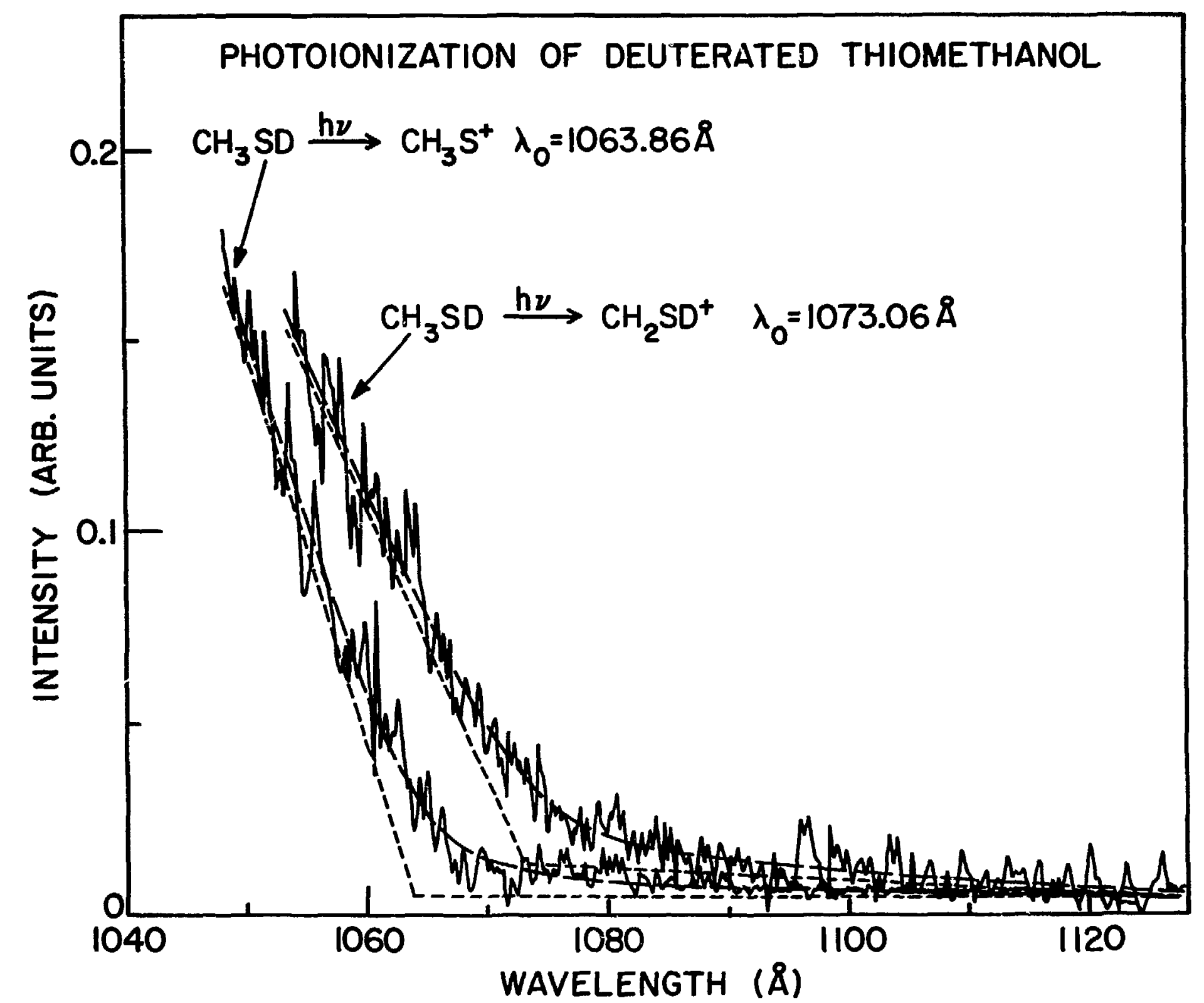

FIg. XII-4. Threshold regions for $\mathrm{M} 48, \mathrm{CH}_{2} \mathrm{SD}^{+}$, and $\mathrm{M} 47, \mathrm{CH}_{3} \mathrm{~S}^{+}$, from the photoionization of $\mathrm{CH}_{3} \mathrm{SD}$. The $\mathrm{CH}_{2} \mathrm{SD}^{+}$is seen to have the lower threshold. 


\title{
XIII. HIGH-RESOLUTION LASER-rF SPECTROSCOPY WITH ATOMIC AND MOLECULAR BEAMS
}

\author{
INTRODUCTION
}

Shortly after completion of our atomic-beam, laser-rf doubleresonarce apparatus, we were strongly urged by members of $R$. W. Field's group at M.I.T. and by others to apply it to small molecular systems. It was pointed out that because of the extremely high resolution, it might be possible to carry out experiments with the precision needed to test ab initio calculations on the alkaline-earth monohalide radicals. These diatomic molecules are characterized by having one electron outside closed shells (in first approximation). This program, begun at the end of 1979, has been very successful. The molecular ground state $\left(\mathrm{X}^{2} \Sigma\right)$ has been investigated in detail for $\mathrm{CaF}, \mathrm{CaCl}$, and $\mathrm{CaBr}$, and the rotational, vibrational, and isotopic dependences have been measured for the spin-rotational and hyperfine (hfs) constants. Many of the parameters measured precisely had never been observed even crudely before. The work has revealed systematic errors in the results of other, cruder methods, and has shown smooth trends in the progression of parameters from one molecule to the next. It has even been possible, with specially developed new techniques, to extend the measurements to excited molecular states in some cases.

Concurrently, a program has been underway for some months to develop an apparatus capable of extending the laser-rf double-resonance technique to beams of atomic and nolecular ions. Because fast beams spend little time interacting with laser or rf flelds, a siow but well focused ion beam is highly desirable. A Colutron model G-1-D ion source capable of decelerating ion beams to $5-10 \mathrm{eV}$ has now been obtained for the system. Fluorescence has been observed from a beam of $\mathrm{Ba}^{+}$.

Good progress has been made in our study of the spin-rotational and hyperfine structure of the alkaline-earth monohalides and the work should be completed during 1982. The program is providing a systematic body of high-precision data covering a select family of small molecules. It is the "single electron outside closed shells" nature of these radicals that makes the data particularly valuable for further development of the ab inito theory. Our successful observation of the hyperfine splittings in $\mathrm{CaCl}$, which no one else had been able to resolve, was gratifying. Messurement of the hfs in several excited states of these molecules was also achieved, and is valuable because of the nearly identical nature of the potential curves for the states involved. 
a. Hfs of the Molecular Ground State $\left(\mathrm{X}^{2} \Sigma\right)$ of $\mathrm{CaCl}$

W. J. Childs, David R. Cok, L. S. Goodman, and G. L. Goodman

Lying halfway between the light $\mathrm{CaF}$ (hfs now reasonably well understo 1) and the heavier CaBr (hfs still very puzzling), the CaCl molecule has been a key candidate for study. A number of laboratories around the world have attempted for several years to observe and study this structure, but all have failed because of the small size of the splittings. Figure XIII-1 shows a high-resolution laser-excitation spectrum of a molecular beam of Cand. Every line in the figure contains four optically unzesolvable hyperfine components. With the new molecularmbeam laser-rf double-resonance technique we have now succeeded in observing the hfs, and have made a precise, systematic study of the rotati nal, vibrational, and isotopic dependence of the splittings of the $x^{2} \Sigma$ molecula ground state. An interesting feature of the work was the observation of a very small magnetic hyperfine anomaly between $\mathrm{Ca}^{35} \mathrm{Cl}$ and $\mathrm{Ca}^{37} \mathrm{Cl}$. Theoretical ab initio work to take advantage of the new results is continuing. An article on this work has been accepted for publication in J. Chem. Phys.

b. New Method for Resolution of Unresolved hfs Components in Optical Spectra: Hfs of the Excited B2 $\Sigma$ State of CaC1

W. J. Childs, David R. Cok, L. S. Goodman, and 0. Poulsen

Although the molecular-beam laser-rf double-resonance technique was successful in determining the hfs splittings within the $x^{2} \Sigma$ ground state of $\mathrm{CaCl}$, the $\mathrm{B} \leftrightarrow \mathrm{X}$ and $\mathrm{A} \leftrightarrow \mathrm{X}$ optical lines still contained unresolved hyperfine structure that prevented measurement of the upper state splittings. This structure is of interest in itself, and a new method was devised to allow us to measure it. A single hfs component of the optical line can be isolated and observed by depopulating the lower levels of all the hfs components by laser pumping, and then repopulating the one of interest by inducing a suitable rf transition. The frequency and level of the rf supplied by the synthesizer is computer controlled with a program that allows switching between different frequencies or turning the rf off for selected times during the computer-controlled laser advance. With the new technique it was shown that the hfs splittings in the excited $\mathrm{B}^{2} \Sigma$ state of $\mathrm{CaCl}$ are not greater than $1 \mathrm{MHz}$. Figure XIII-2 shows the actual resolution of a typical 


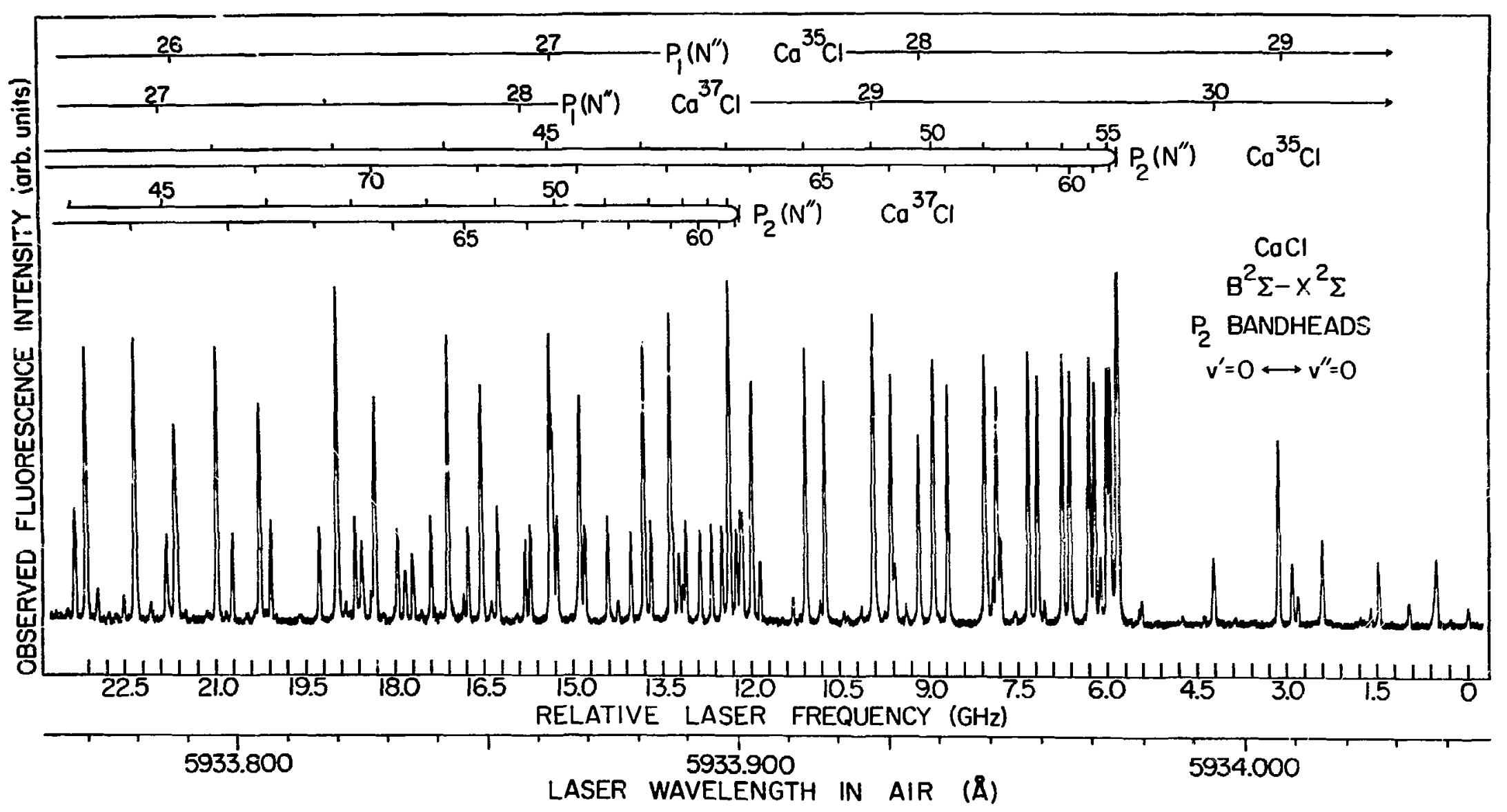

Fig. XIII-1. Sub-Doppler laser-induced fluorescence spectrum of a molecular beam of CaCl near the $\mathrm{B}^{2} \Sigma \leftrightarrow \mathrm{X}^{2} \Sigma(0,0) \mathrm{P}_{2}$ bandheads. The $35 \mathrm{MHz}$ width of each line arises from unresolved hyperfine structure. When rf techniques are combined with fluorescence in a double-resonance scheme, the hfs of the $\mathrm{X}^{2} \sum$ ground state can be resolved and studied in detail for vibrational, rotational, and isotopic dependences. 


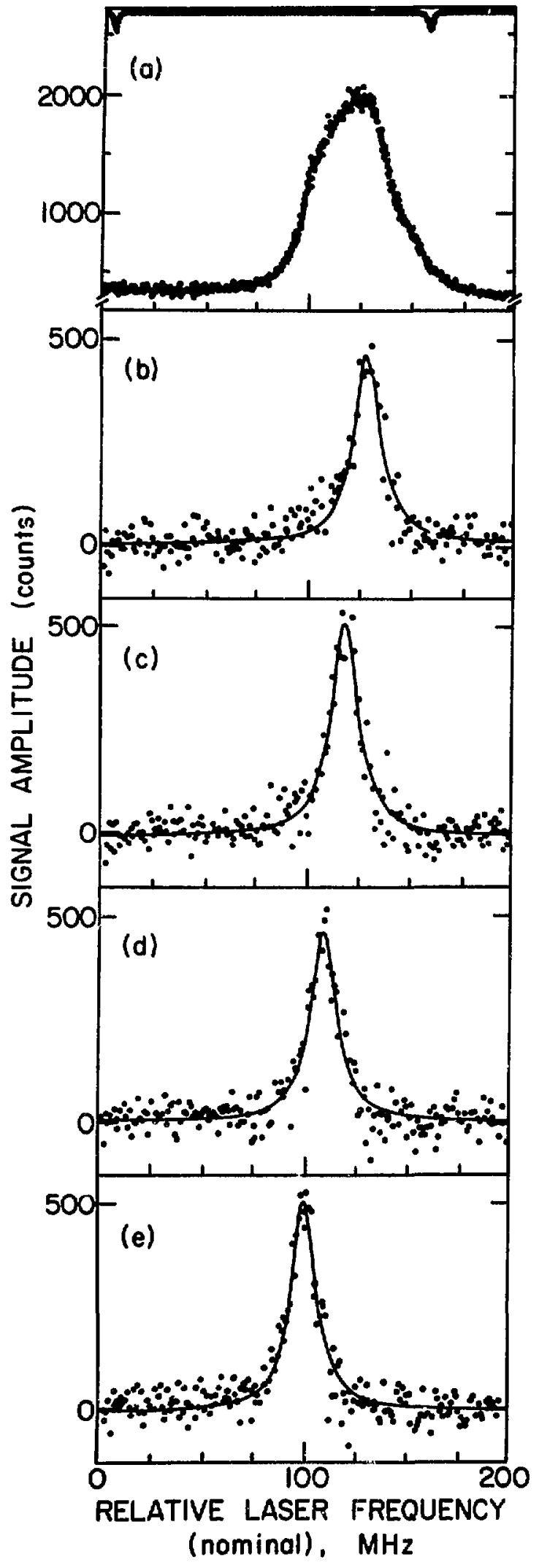

Fig. XIII-2. Use of the new rf-pumped, laser-rf double-resonance technique for the resolution of optically unresolved hfs components within a line. (a) Shows the line profile of a typical line of Fig. XIII-1 under the action of a "pump" laser beam that depletes the lower states of the four hfs transitions ( $I=\frac{3}{2}$ for ${ }^{35} \mathrm{Cl}$ and $37 \mathrm{Cl}$ ) as the laser is swept through the line.

Figure XIII-2(b)-(e) show the increase in fluorescence observed when the four depleted lower levels are repopulated by suitable rf transitions. Combination of the observed splittings in the $\mathrm{B} \leftrightarrow \mathrm{X}$ line with the previously measured $X$ state hfs splittings leads to information on the hfs splittings in the excited B state. 
line in Fig. XIII-1 into its four hfs components by the new double-resonance technique. An article on this work has been published in Phys, Rev. Lett. $\underline{47}$, 1389 (1981).

c. Hfs of the Excited $A^{2} \pi$ State of CaCl

W. J. Childs, David R. Cok, and L. S. Goodman

The new rf-pumped, laser double-resonance technique mentioned in the previous section was subsequently applied to study the hfs of the excited $\mathrm{A}^{2} \pi$ state of $\mathrm{CaCl}$. It too was found to be not more than $1 \mathrm{MHz}$. It is interesting, and not yet understood, ihat both the $A$ and $B$ states have hfs splittings much smaller than for the ground state even though the potential curves for the three states are nearly identical. An article on this work has been accepted for publication in J. Opt. Soc. Am.

d. Spin-Rotational and Hyperfine Structure of the $v=1, x^{2} \Sigma$ state of CaBr

W. J. Childs, David R. Cok, L. S. Goodman, and G. L. Goodman

Efforts in 1980 to extend our study of the hfs of the vibrational ground state of the $x^{2} \Sigma$ state of $\mathrm{CaBr}$ to the $v=1$ manifold proved unsuccessful in that no rf transitions could be found. The problem was associated with an inconsistency between the isotopic and vibrational dependences of the hfs constants. The problem was attacked again in 1981 after dealing successfully with the same situation in $\mathrm{CaCl}$. The sources of the problem were neglect of matrix elements off-diagonal by 2 units in the rotational quantum number $N$, and a small magnetic hyperfine anomaly. The measurements have now been completed, and our knowledge of the rotational and vibrational dependences of the spin-rotation and hfs constants of $\mathrm{CaBr}$ is now on the same precise and complete basis as for $\mathrm{CaF}$ and $\mathrm{CaCl}$. 


\title{
XIV. THEORETILAL ATOMIC PHYSICS
}

\author{
INTRODUCTION
}

The objective of this program is to investigate the role of relativity and many-electron correlation effects in atomic processes, using advanced theoretical techniques such as the relativistic random-phase approximation. The emphasis is on phctoexcitation and photolontzation processes where one can gain important insights into the dynamics of manyelectron interactions. Our current efforts include studies of the spectra of highly stripped lons, where accurate theoretical data are needed for plasma diagnostics in astrophysics and in fusion energy research. We are also studying the autolonization resonances between the first two ${ }^{2} \mathrm{p}$ thresholds of Ar-, $\mathrm{Kr}-$ and Xe-like lons, using the multichannel quantum defect theory. In particular, we are interested in the effects of channel interactions on the positions and profiles of these resonances, as well as those on the absorption spectre of interacting Rydberg series in the bound state region.

Our studies on the effects of relativity and electron correlation in atomic processes consist of four major parts, three of which involve case studies of atomic processes in the discrete, autolonizing and continuous reglons of the spectrum, and one of which deals with the development of a new relativistic many-body theory for atomic calculations.

(1) Atomic Spectra of Highly Stripped Iors. Doubly-excited states formed in beam-foll collision processes usually autolonize very rapidly. However, radiative transitions can take place between doubly-exclted high spin states which are forbidden to autolonize by selection ryles. Fine structure measurements of three-electron quartet states, $(1 s 2 s 2 p)^{4} p$ and $\left(1 s 2 p^{2}\right)^{4} p$, have provided precision tests of relativistic corrections in ab initio atomic structure calculations. We are studying transitions between the quartet states of (1s2snl) and (1s2pnl), where $n l=4 d, 4 f, 5 f$ and $5 g$. Because of the strong centrifugal barrier, these high angular momentum orbitals are pushed, away from the lonic cores, and their couplings with the (1s $2 s)^{3} \mathrm{~s}$ and $(1 \mathrm{~s} 2 \mathrm{p})^{3} \mathrm{p}$ cores are weak. Transition lines between these quartet states have been observed in beam-foil spectra, and they can provide important tests of dynamic core polarization effects.

We have made important progress in calculating forbidden transitions between the ground-state configurations of few-electron systems. Even for highly strlpped lons, the wavelengths of these transitions are still in the visible and the UV region so that they can easily be studied experimentally. As a result, these lines are important diagnostic tools for high temperature plasma in fusion devices. More studles on forbidden transitions are in progress.

(2) Autolonizition Resonances in $\mathrm{Ar}, \mathrm{Kr}$ and $\mathrm{Xe}$ Isoelectronic Sequences. The Beutler-Fano resonances in rare gas atoms have been well studied both theoretically and experfmentally. The spectra consist of sharp ns' resonances superimposed on broad nd' resonances. Recently, the experimental spectrum of $\mathrm{Ba}^{++}$as obtained by a two-stage laser ionization 
technique appears to be quite different from the spectrum of neutral xenon. To understand the mechanism that determines the positions and profiles of these resonances along 18oelectronic sequences, we employ the multichannel quantum defect theory (MQDT) to analyze the resonances in Ar-, Kr- and Xe-11ke 1ons. Elgenchannel data for these analyses are obtalred from ab inttio relativistic random-phase approximation (RRPA) calculations. This technique of combining the MQDT and the RRPA has been very successful in explaining the resonance spectra of neutral rare gas atoms, and it should provide 1mportant insights into the changing correlations and channel interaction effects along isoelectronic sequences.

We are also employing this technique in the study of window resonances arising from Inner shell excitations, nstn'p, in rare gas atoms. In particular, we are tnterested in the energy variations of partial crosssection branching ratios and angular distributions of photoelectrons inside the resonances. Extension of this technique to the study of interacting Rydberg series in the discrete spectra of Ne-like lons is also in progress.

(3) Inner She11 Photolonizations and Orbital Collapses. In the photolonization of the Inner $4 \mathrm{~d}$ shells of $\mathrm{Xe}$, the cross section $1 \mathrm{~s}$ domlnated by the $4 d+\varepsilon f$ transitions, and shows a broad resonance-like structure above the threshold. Thls is caused by a barrier in the effectlve potentlal, which jushes low-energy $f$ orbitals away from the lonic core. Along the isoelectronic sequences, however, the potentlal barrier gradually disappears, and the $f$ orbltals eventually "collapse" towards the origin. When this happens, there w111 be drastlc changes in the spectra, with the disappearance of the resonance-like structure above the threshold, and the appearance of strong absorption lines in the autolonization reglon. Quantitative studies of the collapse phenomena are difficult because the usually small effects such as Hartree-Fock exchanges and electron correlations are greatly amplifled when the potential barrier nearly disappears. Using the RRPA, we are studying the 4d photolonization of Xe-like lons, and, In particular, the change in the spectra along the 1soelectronlc sequence. The RRPA is known to include dominant correlation effects for rare gas systems, and should give Important insights into the nature of f-orbital collapses.

(4) Generallzation of the Relat1vistlc Random-Phase Approximation. The RRPA is known to Include dominant corrolation effects in atomic processes, and is very successful in studying low-energy photolonizations and autolonizations of rare gas atoms. However, the RRPA does not include relaxation effects which appear to be important in group-IIB atoms, and $1 t$ cannot handle double excitations of valence electrons which lead to satellite lines in the absorption spectra. Starting from the time-dependent variational principle and a multiconfiguration ground state, we arrive at a set of generalized RRPA equations which reduce to the usual RRPA equations when there is only one configuration in the ground state. This new technique, the multiconfiguration relativistic random-phase approxipation (MCRRPA), has been applied to the discrete excitations $\left(2 s^{2}\right)^{1} S-(2 s 2 p){ }_{P}$ of $B e-11 k e$ lons by Johnson and Huang. Results on transition energles and absorption oscillator strengths show great improvements over the corresponding, RRPA values. More studies of this method will be carried out. In particular, we are interested in applying this method to photolonization studies, and eventually, to autolonization studies in conjunction with the MQDT technique. 
a. Radiative Transitions Between Quintet States of Four-Electron Ions

H. G. Berry, R. L. Brooks, K. T. Cheng, J. E. Hardis, ${ }^{*}$ and W. Ray

Wavelengths, fine structures and relevant lifetimes for the transitions $\left(1 s 2 s 2 p^{2}\right)^{5} p-\left(1 s 2 p^{3}\right)^{5} s$ in CIII, NIV and ov have been measured using the beam-foil technique. Results are compared with those obtained from a multiconfiguration Dirac-Fock (MCDF) calculation. We found that the Breit interaction plays a crucial role in attaining good agreement between theory and experiment on the fine structures. Furthermore, deviations of the measured wavelengths from the MCDF results provided accurate empirical corrections to the theoretical wavelengths of highly stripped Be-like Icns.

${ }^{*}$ Thesis student, University of Chicago, Chicago, Illinois.

\section{b. Nonrelativistic Total Energy for the (1s3d) ${ }^{1} D$ State of Helium}

C. Froese Fischer ${ }^{*}$ and K. T. Cheng

The nonrelativistic total energy for the $(1 s 3 d)^{1} D$ state of hellum is obtained from the observed $(1 s 2 p)^{1} P-(1 s 3 d)^{1} D$ transition energy and a highly accurate nonrelativistic total energy of the (1s2p) ${ }^{1} P$ state. Corrections arising from relativistic and quantum electrodynamic effects are also taken into account. Our energy value is in good agreement with the ab initio result of Sim et al. For such a comparison, we found that even the motion of the nucleus needs to be taken into account when high accuracy is required.

\footnotetext{
*Vanderbiit University, Nashville, Tennessee.
}

c. Intershe11 Correlation Corrections to the Energy Levels of Few-Electron Ions

K. T. Cheng, C. Froese Flocher ${ }^{*}$ and Y. $-K . \mathrm{KIm}^{\dagger}$

The leading intershell correlation corrections to the energy levels of the $28^{m} 2 p^{k}(m=0-2, k=0-6)$ configurations of three- to nine-electron ions are calculated on the basis of the z-expansion theory. These are leading corrections to the previously published MCDF results of Cheng et al. They

\footnotetext{
*Vanderbilt University, Nashville, Tennessee.

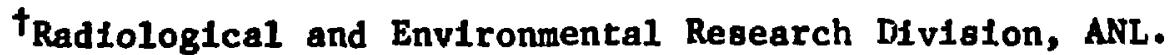


substantially improve the agreement between theory and experiment, and provide accurate spectroscoplc data of highly stripped lons for astrophysics and fustion energy research.

\section{d. Correlation and Relativistic Effects in Spin-Orbit Splittings} K. -N. Huang, ${ }^{*}$ Y. -K. KIm, ${ }^{*}$ K. T. Cheng and J. P. Desclaux ${ }^{\dagger}$

We found a large discrepancy between Dirac-Fock calculations and experiment on the spin-orbit splittings, ${ }^{2} \mathrm{p}_{1 / 2}-{ }^{2} \mathrm{p}_{3 / 2}$, in the ground states of B- and F-like tons. The discrepancy arises from spurious terms which are inherent in the relativistic self-consistent fleld procedure where transition energles are obtalned through separate Initial- and final-state variational calculations. These spurlous terms, though small, can be substantial fractions of the actual level splittings for the fine structures of light atoms, molecules, or lons. They can be removed by a simple procedure, thereby Improving the agreement between theory and experiment on spin-orbit spiltings.

*Radiological and Environmental Research Division, ANL.

tCentre d'Etudes Nucléaires de Grenoble, France.

\section{e. Theoretical Photolonization Parameters for the Rare Gases} K. -N. Huang, * W. R. Johnson ${ }^{\dagger}$ and K. T. Cheng

We have reported a theoretical study of the photoionizations of rare gas atoms $\mathrm{Ar}, \mathrm{Kr}$ and $\mathrm{Xe}$ for photon energles up to $160 \mathrm{eV}$. Partial cross sections, angular distributions and spin polarization parameters of the photoelectrons are calculated with the RRPA technique. These are extensions of previous RRPA results, which are in good agreement with experiment, and they provide complete information on the photolonizations of rare gas atoms.

\footnotetext{
${ }^{*}$ Radiological and Environmental Research Division, ANL.

tUniversity of Notre Dame, Notre Dame, Indiana.
} 
F. Photolonization of Group-IIB Elements

W. R. Johnson, * V. Radojević, * F. Deshmukh * and K. T. Cheng

Theoretical photoionization cross sections, angular distributions and spin-polarization parameters for outer $n s$ and $(n-1) d$ subshells of $\mathrm{Zn}, \mathrm{Cd}$ and Hg are determined above their respective $(n-1) d$ thresholds. Electron correlations and spin-orbit interactions are accounted for by means of the RRPA. Comparisons are made with previous theoretical works and with available experimental measurements. In most cases, the present RRPA results are in excellent agreement with experiment. That RRPA tends to overestimate the absolution cross sections may be an indication of the importance of relaxation effects not included in the RRPA procedure.

\footnotetext{
*University of Notre Dame, Notre Dame, Indiana.
} 


\section{PUBLICATIONS FROM 1 APRIL 1981 THROUGH 31 MARCH 1982}

The list of "journal articles and book chapcers," is classified by topic; the arrangement is approximately that followed in the Tatle of Contents of this Annual Review. The "reports at meetings" include abstracts, sumnaries, and full texts in volumes of proceedings; they are listed chronologically.

\section{A. PUBLISHED JOURNAL ARTICLES AND BOOR CHAPTERS}

1. ELASTIC AND INELASTIC SCATTERING OF $291-\mathrm{MeV}$ PIONS BY ${ }^{9} \mathrm{Be}, \mathrm{Si},{ }^{58} \mathrm{~N} 1$ AND $208 \mathrm{~Pb}$

D. F. Geesaman, C. Olmer, B. Zeidman, R. L. Boudrie,* G. S. Blanpied, $\dagger$

M. J. Devereux ${ }^{\dagger}$ G. R. Burleson, $t$ R. E. Segel, $\neq$ L. W. Swenson,,$\xi$ and

H. A. Thiessen ll

Phys. Rev. C 23, 2635-2647 (June 1981)

2. PION (OR $\triangle$-RESONANCE ABSORPTION IN THE NUCLEAR MEDIUM-A SYNTHESIS OF

PION- AND PHOTO-NUCLEAR REACTIONS

J. P. Schiffer

Comments Nuc1. Part. Phys. 10(5), 243-249 (1981)

3. INCLUSIVE REACTIONS OF PIONS ON NUCLEI

R. D. Mckeown, S, J. Sanders, J. P. Schiffer, H. E. Jackson, M. Paul,

J. R. Specht, E. J. Stephenson, R. P. Redwine, $\pi$ and R. E. Segel $¥$

Phys. Rev. C 24, 211-220 (July 1981)

4. INCLUSIVE PION SCATTERING IN THE $(3,3)$ RESONANCE REGION

S. M. Levenson, D. F. Geesaman, E. P. Colton, ** R. J. Holt, H. E. Jackson, J. P. Schiffer, J. R. Specht, K. E. Stephenson, B. Zeidman, R. E. Segel, $\neq$ P. A. M. Gram, $\|$ and C. A. Gouldingtt

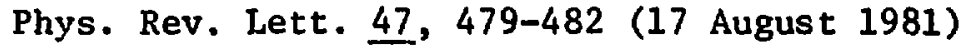

* University of Colorado, Boulder, Colorado and Los Alamos National Laboratory, Los Alamos, New Mexico.

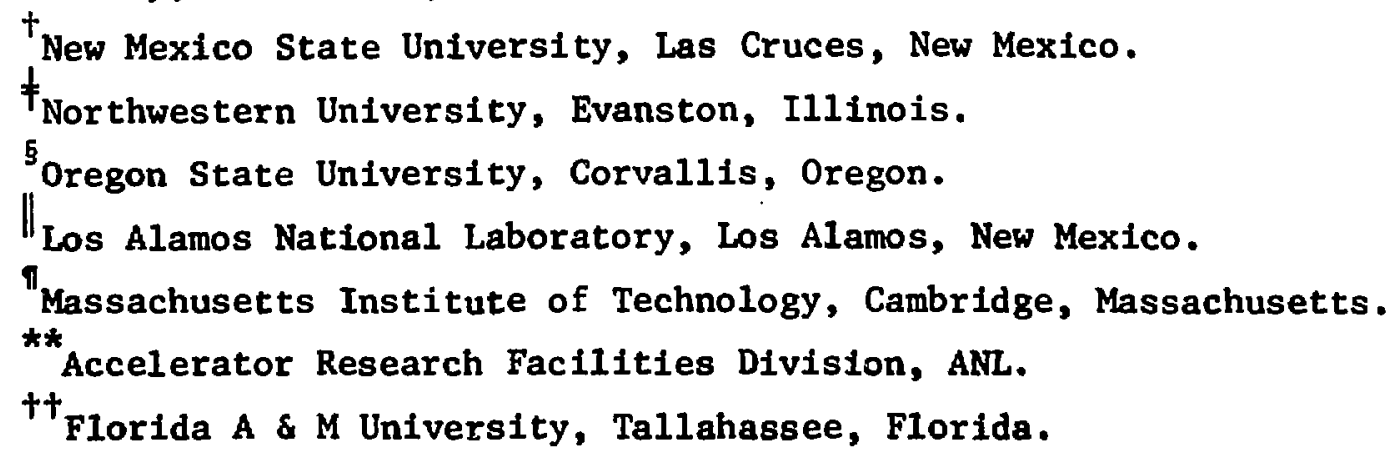


5. ISOSPIN DEPENDENCE OF PION ABSORPTION ON A PAIR OF NUCLEONS

D. Ashery, R. J. Holt, H. E. Jackson, J. P. Schiffer, J. R. Specht,

K. E. Stephenson, R. D. McKeown, ${ }^{*} \mathrm{~J}$. Ungar, ${ }^{*}$ R. E. Sege $1,{ }^{\dagger}$ and

P. Zupranski ${ }^{\dagger}$

Phys. Rev. Lett. 47, 895-898 (28 September 1981)

6. MEASUREMENT OF THE ANGULAR DISTRIBUTION OF TENSOR POLARIZATION IN PIONDEUTERON ELASTIC SCATTERING

R. J. Holt, J. R. Specht, K. Stephenson, B. Zeidman, J. S. Frank, $\neq$

M. J. Leitch, $\neq$ J. D. Moses, $\neq$ E. J. Stephenson, $\$$ and K. M.

Laszewskill

Phys. Rev. Lett. 47, 472-475 (17 August 1981)

Erratum: Phys. Rev. Lett. 47, 1862 (21 December 1981)

7. PHOTON SCATTERING STUDIES OF THE GIANT DIPOLE RESONANCE IN MEDIUM WEIGHT NUCLEI

T. J. Bowles, R. J. Holt, H. E. Jackson, R. M. Laszewski, \| R. D.

Mckeown, A. M. Nathan, $\|$ and J. R. Specht

Phys. Rev, C 24, 1940-1951 (November 1981)

8. YRAST ISOMERS AND VERY HIGH SPIN STATES IN $148,149,151,152$ Dy AND ${ }^{147}$ Gd

B. Haas, ${ }^{\pi}$ D. Ward, ${ }^{* *}$ H. R. Andrews, ${ }^{* *}$ O. Häusser, ${ }^{* *}$ A. J. Ferguson, ${ }^{* *}$

J. F. Sharpey-Schafer, ${ }^{* *}$ T. K. Alexander, ${ }^{* *}$ W. Trautmann, ${ }^{* *}$ D. Horn, ${ }^{* *}$

P. Taras, $+\dagger$ P. Skensved, 扭 T. U. Khoo, R. K. Smither, I. Ahmad, $\S \S$

C. N. Davids, w. Kutschera, S. Levenson, and C. L. Dors \|\|

Nucl. Phys. A362, 254-300 (1 June 1981)

9. FEEDING OF HIGH-SPIN PARTICLE YRAST STATES BY COLLECTIVE STRUCTURES IN THE CONTINUUM

P. Chowdhury, J. Borggreen, T. L. Khoo, I. Ahmad, $\$ \S$ R, K. Smither,

S. R. Faber, \|\| P. J. Daly, \|\| C. L. Dors, \|\| and J. W:1son\|\| Phys. Rev. Lett. 47, 778-781 (14 September 1981)

10. SHELL MODEL YRAST STATES OF THE N=83 NUCLEUS ${ }^{150}$ Ho

J. Wilson, $\| \mid$ Y. H. Chung, \|\| S. R. Faber, \|\| A. Pakkanen, \|\| P. J. Daly, \|\|

I. Ahmad, $\$ \xi$ P. Chowdhury, T. I. Khoo, R. D. Lawson, and R. K. Smither Phys. Lett. 103B, 413-416 (6 August 1981)

\footnotetext{
${ }^{*}$ California Institute of Technology, Pasadena, California.

${ }^{\dagger}$ Northwestern University, Evanston, Illinois.

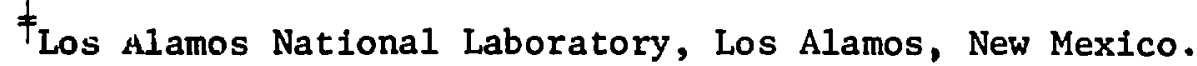

${ }^{\S}$ Indiana University, Bloomington, Indiana.

University of Illinois, Urbana, Illinois.

"Centre de Recherches Nucléaires, Strasbourg, France.

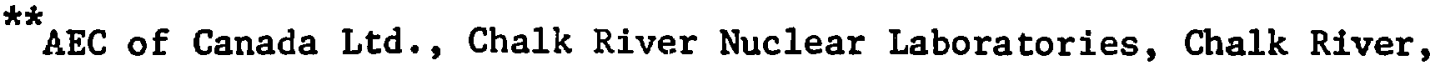
Ontario, Canada.

tt University of Montreal, Montreal, Quebec, Canada.

俦 Queen's University, Kingston, Ontario, Canada.

${ }^{\$ \S}$ Chemistry Division, ANL.

\|\|$_{\text {Purdue University, West Lafayette, Indiana. }}$
} 
11. ROTATIONAL-VIBRATIONAL BAND STRUCTURE IN ${ }^{155} \mathrm{Sm}$

K. Schreckenbach, ${ }^{*}$ A. I. Namenson, ${ }^{*}$ W. F. Davidson, ${ }^{*}$ T. von Egidy, ${ }^{*}$

H. G. Börner, * J. A. Pinston, * R. K. Smither, D. D. Warner, ${ }^{*}$ R. F. Casten, ${ }^{\dagger}$ M. L. Stelts, ${ }^{\dagger}$ D. H. White, $\dagger^{\dagger}$ and W. Stöff $1^{\S}$ Nucl. Phys. A376, 149-182 (1982)

12. INTERMEDIATE-STRUCTURE FUSION RESONANCES OBSERVED IN THE RADIATIVE CAPTURE OF ${ }^{12} \mathrm{C}$ BY $12 \mathrm{C}$

A. N. Nathan, $\|$ A. M. Sandorfi, $\dagger$ and T. J. Bowles

Phys. Rev. C 24, 932-943 (September 1981)

13. FUSION of ${ }^{24} \mathrm{Mg}+{ }^{24} \mathrm{Mg}$

C. M. Jachcinsk1, D. G. Kovar, R. R. Betts, П C. N. Davids, D. F. Geesaman, C. Olmer, M. Paul, S. J. Sanders, and Y. L. Yntema Phys. Rev. C 24, 2070-2075 (November 1.981)

14. FUSION CROSS SECTION BEHAVIOR FOR ${ }^{12} \mathrm{C}+{ }^{24} \mathrm{Mg}$ AND ${ }^{12} \mathrm{C}+{ }^{26} \mathrm{Mg}$

K. Daneshvar, D. G. Kovar, S. J. Krieger, ${ }^{* *}$ and K. T. R. Daviestt Phys. Rev. C 25, 1342-1354 (March 1982)

15. \& DETERMINATIONS FOR ${ }^{12} \mathrm{C}+{ }^{12} \mathrm{C}$

H. T. Fortune, 邦 S. C. Headley, 㭋 A. Spadafora, 㭋 J. Sweet, 玤 s. LaFrance, $\neq \neq$ M. E. Cobern, $\neq \neq$ G. E. Moore, $\neq \neq$ M. Newcomer, $\neq \neq$ E. Wallash, $\neq \neq$ L. Bland, $\neq \neq$ J. Gilfoyle, $\neq \neq$ R. Gilman, $\neq \neq$ M. Carchidi, $\neq \neq$ G. S. Stephans, $\neq \neq$ L. R. Greenwood, J. R. Erskine, R. E. Segel, T. H. Braid, and K. Raghunathan

Phys. Lett. 108B, 95-97 (14 January 1982)

16. FRAGMENTATION OF ${ }^{16} 0$ AND ${ }^{18} 0$ PROJECTILES

S. L. Tabor, $\S \S$ L. C. Dennis, $\$ \S$ K. W. Kemper, $\S \S$ J. D. Fox, $\S \S$ K. Abdo, $\S \S$

G. Neuschaefer, $\$$ D. G. Kovar, and H. Ernst

Phys. Rev. C 24, 960-965 (September 1981)

17. NEW METHOD FOR FOCUSING $x$ RAYS AND GAMMA RAYS

R. K. Smither

Rev. Sci. Instrum.' 53, 131-141 (February 1982)

* Institut Laue-Langevin, Grenoble, France.

${ }^{\dagger}$ Brookhaven National Laboratory, Upton, New York.

‡oregon College of Education, Monmouth, Oregon.

$\xi_{\text {IU München, West Germany. }}$

University of Illinois, Urbana, Illinois.

"Chemistry Division, ANL.

** University of California, Lawrence Livermore National Laboratory, Livermore, California.

${ }^{t \dagger}$ Oak Ridge Netional Laboratory, Oak Ridge, Tennessee.

螼University of Pennsylvania, Philadelphia, Pensylvania.

${ }^{\S}$ Florida State University, Tallahassee, Florida. 
18. THE $(d, t)$ REACTION ON CHLORINE ISOTOPES AT $23 \mathrm{MeV}$

C. M. Bhat, * N. G. Puttaswamy, ${ }^{*}$ and J. L. Yntema J. Phys. G I, 1529-1538 (1981)

19. OBSERVATION OF THE CAPTURE REACTION ${ }^{2} \mathrm{H}(\alpha, \gamma)^{6} \mathrm{~L} 1$

R. G. H. Robertson, $t$ P. Dyer, $t$ R. A. Warner, $t$ R. C. Melin, $t$ T. J.

Bowles, A. B. McDonald, $\neq$ G. C. Ball, $\neq$ W. G. Davies, $\neq$ and E. D. Earle $\neq$ Phys. Rev. Lett. 47, 1867-1870 (28 December 1981)

20. NEUTRINOS FROM A STANDARD SOLAR MODEL

B. W. Filippone and David N. Schramm 5 Astrophys. J. 253, 393-398 (1 February 1982)

+ Ph.D. Thesis, University of Chicago

21. COIILD $+\frac{1}{3}$ e STABLE PARTICLES BE CONSISTENT WITH PRESENT OBSERVATIONS?

John P. Schiffer

Phys. Rev. Lett. 48,213 (18 January 1982)

22. THE SENSITIVE RADIUS-SPATIAL LOCALIZATION IN HEAVY-ION REACTION ANALYSES

M. H. Macfarlane $\|$ and Steven C. Pieper

Phys. Lett. 103B, 169-172 (23 July 1981)

23. $\left(\pi \mathrm{h}_{11 / 2}\right)^{\mathrm{n}}$ STATES EXPECTED IN ${ }_{68}^{150} \mathrm{Er}_{82},{ }_{69}^{151} \operatorname{Tm}_{82}$ AND ${ }_{70}^{152} \mathrm{Yb}_{82}$

R. D. Lawson

Z. Phys. A $303,51-61$ (November 1981)

24. HIGH-LYING $0^{+}$AND $3^{-}$LEVELS IN ${ }^{12} \mathrm{C}$

S. S. Hanna, $\pi$ W. Feldman, "M. Suffer:t, $\pi$ and D. Kurath

Phys. Rev. C 25, 1179-1186 (March 1982)

25. PARITY-VIOLATING MATRIX ELEMENT IN ${ }^{10} \mathrm{~B}$

D. Kurath and $W$. Teeters

Phys. Lett. 101B, 5-9 (30 April 1981)

26. EXACT GROUND STATES OF FEW-BODY NUCLEI WITH AND WITHOUT THREE-BODY FORCES

John G. Zabolitzky, K. E. Schmidt, ** and M. H. Kalos**

Phys. Rev. C 25, 1111-1113 (February 1982)

27. NTUCLEAR SATURATION FROM TWO-NUCLEON POTENTIALS

B. D. Day

Phys. Rev. Lett. $47,226-229$ (27 July 1981)

\footnotetext{
*Bangalore University, Bangalore, India.

†ichigan State University, East Lansing, Michigan.

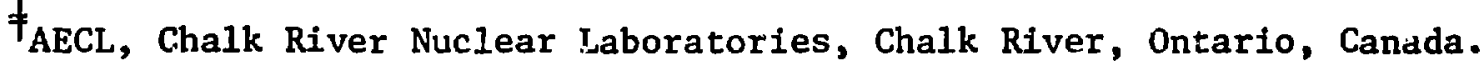

SUniversity of Chicago, Chicago, Illinols.

Indiana University, Bloomington, Indiana.

"Stanford University, Stanford, California.

${ }^{\star \star}$ Courant Institute of Mathematical Sciences, New York, New York.
} 
28. COUPLED-CLUSTER CALCULATION FOR NUCLEAR MATTER AND COMPARISON WLTH THE HOLE-LINE EXPANSION

B. D. Day and John G. Zabolitzky Nucl. Phys. A366, 221-244 (17 August 1981)

29. THREE-BODY CORRELATIONS IN NUCLEAR MATTER

B. D. Day

Phys. Rev. C 24, 1203-1271 (September 1981)

30. THE AHARONOV-BOHM EFFECT: WHY IT CANNOT BE ELIMINATED FROM QUANTUM MECHANICS

Murray Peshkin

Phys. Reports $80(6), 375-386$ (December 1981)

31. ON THE RELATION BETWEEN THE METHOD OF COUPLED CHANNELS AND THE TRUNCATED CHANNEL COUPLING ARRAY FORMALISM FOR REARRANGEMENT REACTIONS

M. Kawai, M. Ichimura, * and N. Austern'

2. Phys. A $303,215-226$ (November 1981)

32. THE POTENTIAL MODEL OF COLORED QUARKS: SUCCESS FOR SINGLE-HADRON

STATES, FAILURE FOR HADRON-HADRON INTERACTIONS

0 . W. Greenberg $\neq$ and Harry J. Lipkin

Nuc1. Phys. A370, 349-364 (16 November 1981)

33. MODEL-INDEPENDENT ANALYSIS OF EXPERIMENTAL BARYON MAGNETIC MOMENTS

Harry J. Lipkin

Phys. Rev. D 24, 1437-1440 (1 September 1981)

34. WHY THE $\Sigma$ AND $\Xi$ HYPERFINE SPLITTINGS ARE DIFFERENT

Isaac Cohen ${ }^{5}$ and Harry J. Lipkin

Phys. Lett. 106B, 119-124 (29 October 1981)

35. IMPORTANCE OF THE $k_{\eta}$ AND $k_{\eta}$ ' DECAY MODES IN UNDERSTANDING CHARMED AND OTHER MESON DECAYS

Harry J. Lipkin

Phys. Rev. Lett. 46, 1307-1310 (18 May 1981)

36. FROM QUARKS TO THE BIG BANG

Harry J. Lipkin

New Scientist 90, 276-277 (30 April 1981)

37. CONSTRAINTS ON COMPOSITE MODELS OF QUARKS AND LEPTONS

Harry $J$. Lipkin

Phys. Lett. 103B, 440-444 (6 August 1981)

\footnotetext{
* University of Tokyo, Tokyo, Japan.

†Uiversity of Pittsburgh, Pittsburgh, Pennsylvania.

†niversity of Maryland, College Park, Maryland.

$\$$ Weizmann Institute of Science, Rehovot, Israel.
} 
38. GLUEBALLS VERSUS QUARKONIUM-FLAVOR SYMMETRY SIGNATURES Harry J. Lipkin Phys. Lett. 109B, 326-330 (25 February 1982)

35. THE E IS NOT A GLUEBALL-BUT FLAVOR SYMMETRY SHOWS HOW TO FIND THEM Harry J. Lipkin

Phys. Lett. 106B, 114-118 (29 October 1981)

40. ASPECTS OF SECONDARY ION EMISSION

A. R. Krauss* and V. E. Krohn

Chap. 5 in Mass Spectrometry, ed. by R. A. W. Johnstone (The Royal Soclety of Chemistry, London, 1981), Vol. 6, pp. 118-152

41. COMMENTS ON THE SURFACE-EXCITATION MODEL

V. E. Krohn

Surf. Sci. 111, L744-L746 (1981)

42. INTERACTIONS OF FAST (MeV) MOLECULAR IONS WITH MATTER

E. P. Kanter

Comments At. Mol. Phys. $11(2), 63-74$ (1981)

43. Uv-LASER PHOTOFRAGMENTATION OF A $2-\mathrm{MeV} \mathrm{H}_{2}{ }^{+}$BEAM

A. K. Edwards, R. Kutina, N. Cue, D. S. Gemmell, K. Inglis, E. P. Kanter, and B. J. Zabransky

Phys. Rev. A 23, 2724-2726 (May 1981)

44. INTERACTIONS OF FAST $\mathrm{HeH}^{+}$BEAMS WITH MATTER

P. J. Cooney, D. S. Gemme11, W. J. Pietsch, A. J. Ratkowski, Z. Vager, and B. J. Zabransky

Phys. Rev. A 24, 746-757 (August 1981)

45. CONTRIBUTION OF FIELD-IONIZED RYDBERG ATOMS IN OBSERVATIONS ON CONVOY ELECTRONS

Z. Vager, B. J. Zabransky, D. Schneider, E. P. Kanter, Gu Yuan Zhuang, and D. S. Gemme11

Phys. Rev. Lett. 48, 592-595 (1 March 1982)

46. $2 s-2 p$ TRANSITIONS IN HELIUMLIKE IONS

R. DeSerio, H. G. Berry, R. L. Brooks, J. Hardis, A. E. Livingston, ${ }^{\dagger}$ and S. J. Hinterlong ${ }^{t}$

Phys. Rev. A 24, 1872-1888 (October 1981)

47. PERIODIC WAVELENGTH DISPERSION IN A SCANNING UV MONOCHROMATOR

R. DeSerio

App1. Optics 20, 1781-1784 (15 May 1981)

48. MEASIREMENTS AND A MODEL FOR THE ENHANCEMENT OF LYMAN- $\alpha$ RADIATION FOLLOWING FOIL-INDUCED DISSOCIATION OF MOLECULAR IONS

R. L. Brooks and H. G. Berry

Phys. Rev. A 25, 161-168 (January 1982)

\footnotetext{
${ }^{\star}$ Chemistry nivision, ANL.

${ }^{+}$University of Notre Dame, Notre Dame, Indiana.
} 


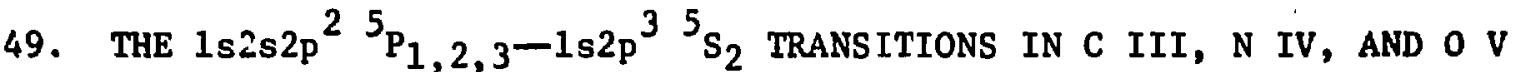
H. G. Berry, R. L. Brooks, K. T. Cheng, J. E. Hardis, and W. Ray Physica Scripta 25, 391-393 (February 1982)

50. MOLECULAR EFFECTS IN BEAM-FOIL COLLISION-INDUCED ALIGNMENT OF He I

T. J. Gay, H. G. Berry, and R. DeSerio

Phys. Rev. A 23, 1761-1774 (April 1981)

51. ENERGY DEPENDENCE OF ALIGNMENT IN FOIL COLLISION-EXCITED $n=3$ STATES OF He $\mathrm{I}$

T. J. Gay, H. G. Berry, R. DeSerio, H. P. Garnir, R. M. Schectman,*

N. Schaffel, ${ }^{*}$ R. D. Hight, ${ }^{\dagger}$ and D. J. Burnst

Phys. Rev. A 23, 1745-1760 (Apri1 1981)

+ Ph.D. Thesis, University of Chicago, 1981

52. X $\alpha$ CALCULATIONS AND SPECTROSCOPIC STUDIES OF TRIRUTHENIUM AND TRIOSMIUM DODECACARBONYLS

Bernard Delley, $\neq$ Mark C. Manning, $\neq$ D. E. Ellis, $\neq$ Joseph Berkowitz, and William C. Trogler

Inorg. Chem. 21, 2247-2253 (1982)

53. PHOTOIONIZATION OF ATOMIC IODINE AND ATOMIC TELLURIUM

J. Berkowitz, C. H. Batson, and G. L. Goodman

Phys. Rev. A 24, 149-160 (July 1981)

54. DOUBLE RESONANCE, FLUORESCENCE SPECTROSCOPY, AND HYPERFINE STRUCTURE IN $\operatorname{Pr} I$

W. J. Childs and L. S. Goodman

Phys. Rev. A 24, 1342-1349 (September 1981)

55. INVESTIGATION OF UNRESOLVED hfS SPLITTINGS IN THE ${ }^{2} \Sigma$ STATE OF CaCl WITH USE OF $\mathrm{r} f-P U M P E D-$ LASER DOUBLE-RESONANCE SPECTROSCOPY

W. J. Childs, David R. Cok, L. S. Goodman, and O. Poulsen

Phys. Rev. Lett. $47,1389-1391$ (9 November 1981)

56. HYPERFINE AND SPIN-ROTATIONAL STRUCTURE OF CaBr $\mathrm{X}^{2} \Sigma(\mathrm{v}=0)$ BY MOLECULAR-BEAM LASER-rf DOUBLE RESONANCE

W. J. Childs, David R. Cok, G. L. Goodman, and L. S. Goodman

J. Chem. Phys. 75, 501-507 (15 July 1981)

57. DETERMINATION OF DIPOLE AND QUADRUPOLE HYPERFINE STRUCTURE IN THE EXCITED B2 $\Sigma$ STATE OF $\mathrm{Ca}{ }^{79} 9_{\mathrm{Br}}$ AND $\mathrm{Ca}{ }^{8} 1_{\mathrm{Br}}$

W. J. Childs, David R. Cok, and L. S. Goodman

Can. J. Phys. 59, 1308-1312 (1981)

58. PHOTOIONIZATION OF GROUP-IIB ELEMENTS

W. R. Johnson, $\$$ V. Radojević, $\$$ Pranawa Deshmukh, $\$$ and $K$. T. Cheng Phys. Rev. A 25, 337-345 (January 1982)

\footnotetext{
*University of Toledo, Toledo, Ohio.

tUniversity of Nebraska, Lincoln, Nebraska.

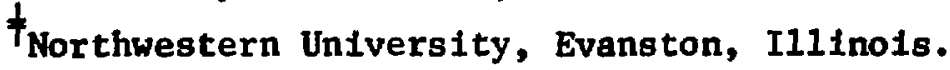

${ }^{\xi}$ University of Notre Dame, Notre Dame, Indiana.
} 
59. A NOTE ON ESTIMATING THE $1 \mathrm{~s} 3 \mathrm{~d}^{1} \mathrm{D}$ EXACT, NONRELATIVISTIC TOTAL ENERGY FOR HELIUM

Charlotte Froese Fischer ${ }^{*}$ and Kwolk-tsang Cheng

J. Phys. B 15, 337-339 (14 February 1982)

60. INTERSHELL CORRELATION CORRECTIONS TO THE ENERGY LEVELS OF THE $n=2$ STATES OF L1-LIKE TO F-LIKE IONS

K. T. Cheng, C. Froese Fischer, ${ }^{*}$ and Y.-K. Kim ${ }^{\dagger}$

J. Phys. B $15,181-189$ (1982)

61. DEPTH DISTRIBUTION OF 20-keV HELIUM ION IRRADIATION INDUCED CAVITIES

IN NICKEL

G. Fenske, $\neq$ S. K. Das, and M. Kaminsky

J. Nuc1. Mater. 103 \& $104,1231-1235$ (1981)

62. EXPERIMENTAL AND CALCULATED DEPTH DISTRIBUTIONS OF DAMAGE AND PROJECTED RANGES OF $20-\mathrm{keV} 4 \mathrm{He}$ IONS IN NICKEL

G. Fenske, $\neq$ S. K. Das, M. Kaminsky, G. Miley,, B. Terreault, $\|$ G. Abel, $\|$ and J. P. Labrie

J. App1. Phys. 52, 3618-3621 (May 1981)

63. SPUTTERING OF TITANIUM CARBIDE COATINGS UNDER $\mathrm{D}^{+}$AND ${ }^{4} \mathrm{He}^{+}$ION BOMBARDMENT: TOTAL SPUTTER YIELDS AND ANGULAR DISTRIBUTION

M. Kaminsky and R. Nielsen

Thin Solid Films 83 , 107-114 (1981)

64. SURFACE ROUGHNESS FACTOR MEASUREMENTS OF 304 AND 316 STAINLESS STEELS WITII HELIUM ION IRRADIATION

S. Maeda, ${ }^{* *}$ M. Mohri, ${ }^{* *}$ M. Hashiba, ${ }^{* *} T$. Yamashina, ${ }^{* *}$ and M. Kaminsky J. Nuc1. Mater. $103 \& \underline{104}, 445-449$ (1981)

65. SURFACE EROSION OF TiC COATINGS UNDER ${ }^{4} \mathrm{He}^{+}$AND $\mathrm{D}^{+}$ION IRRADIATION

A. S. Rao and M. Kaminsky

Thin Solid Films 83 , 93-106 (1981)

66. SUPPRESSION OF REPETITIVE SURFACE EXFOLIATION OF INCONEL 625 IMPLANTED SEQUENTIAZLY WITH HELIUM IONS OF DIFFERENT ENERGIES (20-100 keV)

A. S. Rao, J. L. Whitton, and M. Kaminsky

J. Nucl. Mater. $103 \& 104,397-401$ (1981)

*Vanderbilt University, Nashville, Tennessee.

${ }^{\dagger}$ Radiological and Environmental Research Division, ANL.

\# Materials Science Division, ANL.

${ }^{5}$ University of Illinois, Urbana, Illinois.

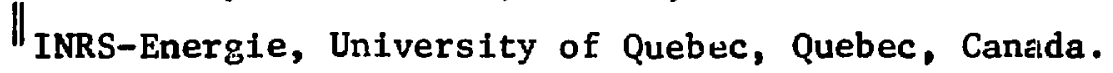

"University of Montreal, Montreal, Quebec, Canada.

** Hokkaido University, Sapporo, Japan. 


\section{B. PUBLISHED REPORTS AT MEETINGS}

Proceedings of the Fifth Tandem Conference, Catania, Italy, 9-12 June 1980

1. ACCELERATOR MASS SPECTROMETRY AND RADIOISOTOPE DETECTION AT THE ARGONNE FN TANDEM FACILITY

Walter Heining, Walter Kutschera, Michael Paul, Robert K.

Smither, Edward J. Stephenson, and Jan L. Yntema Nucl. Instrum. Methods 184, 247-268 (15 May 1981)

+ Program of the Conference, organized by G. Calvi et al. (University of Catania, 1980)

2. THE ARGONNE TANDEM AS INJECTOR TO A SUPERCONDUCTING LINAC

J. L. Yntema, P. K. Den Hartog, W. Henning, and W. Kutschera Nuc1. Instrum. Methods 184, 233-238 (15 May 1981)

+ Program of the Conference

Proceedings of 50th Nobel Symposium on Nuclei at Very High Spin, Örenäs, Sweden, 23-27 June 1980

3. VERY HIGH SPIN YRAST STATES AND THE LINK TO THE CONTINUUM T. L. Khoo, J. Borggreen, P. Chowdhury, I. Ahmad, * R. K. Smither, S. R. Faber, ${ }^{\dagger}$ P. J. Daly, ${ }^{+}$C. L. Dors, ${ }^{+}$and J. Wilson ${ }^{\dagger}$ Physica Scripta 24(1:2), 283-289 (1981)

Neutrino Physics and Astrophysics (Proceedings of International Conference, Erice, Italy, 23-28 June 1980), ed. by Ettore Fiorini (Plenum, New York, 1982)

4. PARITY VIOLATION IN NUCLEI

R. G. H. Robertson pp. 219-238 + discussinn on p. 239

Proceedings of the Symposium on Perspectives in Electro- and Photo-Nuclear Physics, Saclay, France, 29 September-3 October 1980

5. DESIGN OBJECTIVES FOR A GeV C.W. ELECTRON ACCELERATOR

Y. Cho, $\neq$ R. J. Holt, H. E. Jackson, T. K. Khoe, $\neq$ and $G$. Mavrogenes*

Departement de Physique Nucleaire Report DPh-N/HE/81/2

(CEN Saclay, 1981), pp. 41-43

6. DEVELOPMENT OF A DEUTERON TENSOR POLARIMETER SUITABLE FOR STUDY OF e-d AND $\pi-d$ ELASTIC SCATTERING

R. J. Holt, R. M. Laszewski, J, R. Specht, E. J. Stephenson,

K. E. Stephenson, J. S. Frank,, M. J. Leitch, $\$$ and J. D. Moses $\S$ Nuc1. Phys. A358, 273c-274c (13 April 1981)

\footnotetext{
*Chemistry Division, ANL.

${ }^{\dagger}$ Purdue University, West Lafayette, Indiana.

\# Accelerator Research Facilities Division, ANL.

${ }^{5}$ Los Alamos National Laboratory, Los Alamos, New Mexico.
} 
Perspectives in Electro- and Photo-Nuclear Physics, Saclay, SeptemberOctober 1980 (cont'd.)

7. COMPARISON OF PION AND PHOTON INDUCED REACTIONS ON COMPLEX NUCLEI

R. D. Mckeown, J. P. Schiffer, H. E. Jackson, M. Paul, S. J. Sanders, J. R. Specht, E, J. Stephenson, R. P. Redwine, *

R. E. Segel ${ }^{\dagger}$ J. Arends, $\neq$ J. Eyink, $\neq$ H. Hartmann, $\neq$ A. Hegerath,$\neq$

B. Mecking, $\neq$ G. Nöldeke, $\neq$ and $H$. Rost $\neq$ Nucl. Phys. A358, 411c-412c (13 April 1981)

1980 Conference on the Application of Accelerators in Research and Industry, Denton, Texas, 3-5 November 1980

8. FOIL DISSOCIATION OF FAST MOLECULAR IONS INTO ATOMIC EXCITED STATES

H. G. Berry, T. J. Gay, and R, L. Brooks

IEEE Trans. Nucl. Sci. NS-28, 1174-1177 (Apri1 1981)

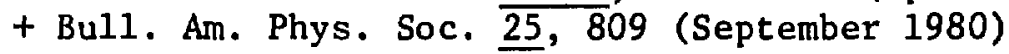

9. THE ARGONNE SUPERCONDUCTING HEAVY-ION LINAC

L. M. Bollinger

Bul1. Am. Phys. Soc. 25, 767 (September 1980)

10. USING AN ELECTROSTATIC ACCELERATOR TO DETERMINE THE STEREOCHEMICAL STRUCTURES OF MOLEC.JLAR IONS

Donald S. Gemmeli

IEEE Trans. Nucl. Sci. NS-28, 1168-1173 (April 1981)

+ Bull. Am. Phys. Soc. $\underline{25}, 809$ (September 1980)

11. NUCLEAR CROSS SECTIONS FOR LIGHT IONS ON ${ }^{6} \mathrm{Li}$

R. E. Holland, A. J. Elwyn, C. N. Davids, L. Meyer-Schützmeister,

J. E. Monahan, F. P. Mooring, and W. Ray, Jr.

IEEE Trans. Nucl. Sci. NS-28, 1344-1349 (April 1981)

+ Bull. Am. Phys. Soc. 25,775 (September 1980)

12. THRESHOLD PHOTONEUTRON ANGULAR DISTRIBUTION AND POLARIZATION STUDIES OF NUCLEI

R. J. Holt

IEEE Trans. Nucl. Sci. NS-28, 1279-1285 (April 1981)

+ Bull. Am. Phys. Soc. 25, 756 (September 1980)

13. PHYSICS WITH FAST MOLECULAR-ION BEAMS

Elliot P. Kanter

IEEE Trans. Nuc1. Sci. NS-28, 1162-1167 (April 1981)

+ Bul1. Am. Phys. Soc. 25, 765 (September 1980)

14. RESONANT STRUCTURES IN HEAVY-ION REACTIONS

S. J. Sanders, W. Henning, H. Ernst, J. F. Geesaman, C.

Jachcinski, D. G. Kovar, M. Paul, and J. P. Schiffer

IEEE Trans. Nucl. Sci. NS-28, 1246-1249 (April 1981)

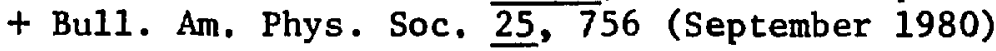

*Massachusetts Institute of Technology, Cambridge, Massachusetts.

${ }^{\dagger}$ Northwestern University, Evanston, Illinols,

'University of Bonn, Bonn, West Germany. 
Application of Accelerators in Research \& Industry, Denton, Nov. 1980 (cont'd.)

15. CARBON STRIPPER FOILS FOR HEAVY ION ACCELERATORS

G. E. Thomas

IEEE Trans. Nucl. Sci. NS-28, 1584-1587 (April 1981)

+ Bull. Am. Phys. Soc. $\underline{25}, 763$ (September 1980)

Proceedings of the Nuclear Physics and Solid State Physics Symposium, New Delhi, India, 11-15 December 1980 (Department of Atomic Energy, India, 1981)

16. (d, ${ }^{3} \mathrm{He}$ ) REACTION ON POTASSIUM ISOTOPES AT $22.8 \mathrm{MeV}$

C. M. Bhat,* M. Raja Rao,* N. G. Puttaswamy,* and J. L. Yntema pp. 199-201

Fifth International Conference on Ion Beam Analysis, Sydney, Australia, 16-20 February 1981

17. THE "COILOMB-EXPLOSION" TECHNIQUE FOR DETERMINING GEOMETRICAL STRUCTURES OF MOLECULAR IONS

Donald S. Gemme11

Nucl. Instrum. Methods 191, 425-431 (1981)

+ Ion Beam Analysis (Proceedings of the Conference), ed. by J. R. Bird and G. J. Clark (North-Holland, Amsterdam, 1981), pp. 425-431 [Reprinted from Nuc1. Instrum. Methods 191, 425 (1981)]

1981 Particle Accelerator Conference - Accelerator Engineering and Technology, Washington, D.C., 11-13 March 1981

18. THE SUPERCONDUCTING HEAVY-ION LINAC AT ARGONNE

$J$. Aron, $t$ R. Benaroya, L. M. Bollinger, B. E. Clifft, $+K$. W. Johnson, $\dagger$ J. M. Nixon, $\dagger$ P. Markovich, ${ }^{+}$R. C. Pardo, and K. W. Shepard

IEEE Trans. Nucl. Sci. NS-28(3), 3458-3460 (June 1981)

+ Bul1. Am. Phys. Soc. 26,166 (February 1981)

19. A CONCEPTUAL DESIGN OF A LINAC-STRETCHER RING TO OBTAIN A 2-GeV CONTINUOUS ELECTRON BEAM

Yanglai Cho, $\neq$ Roy J. Holt, Harold E. Jackson, Tat K. Khoe, $\neq$ and George S. Mavrogenes ${ }^{\dagger}$

IEEE Trans. Nuc1. Sci. NS-28(3), 2119-2121 (June 1981)

+ Bull. Am. Phys. Soc. 26, 102 (February 1981)

20. THE DESIGN OF THE ARGONNE DOUBLE-SIDED MICROTRON

Tat K. Khoe, $\neq$ Yanglai Cho,f Roy J. Holt, Harold E. Jackson, and George S. Mavrogenes ${ }^{\dagger}$

IEEE Trans. Nuc1. Sci. NS-28(3), 2077-2079 (June 1981)

+ Bull. Am. Phys. Soc. 26, 99 (February 1981)

*Bangalore University, Bangalore, India.

${ }^{\dagger}$ Chemistry Division, ANI.

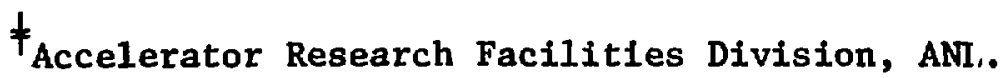


Particle Accelerator Conference, Washington, D.C., March 1981 (cont'd.)

21. $\mathrm{Xe}^{+n}-\mathrm{Xe}^{+\boldsymbol{n}}$ CHARGE EXCHANGE EXPERIMENT USING A SPLIT-BEAM AND BEAM INTERSECTION TECHNIQUE

Michael G. Mazarakis, * H. Gordon Berry, and Eugene P. Colton* IEEE Trans. Nuc1. Sc1. NS-28(3), 3184-3186 (June 1981)

+ Bull. Am. Phys. Soc. $\underline{26}, 152$ (February 1981)

22. DEVELOPMENT OF SUPERCONDUCTING RESONATORS FOR THE ARGONNE

HEAVY-ION LINAC

K. W. Shepard

IEEE Trans. Nuc1. Sci. NS-28(3), 3248-3250 (June 1981)

+ Bul1. Am. Phys. Soc. 26,155 (February 1981)

23. A SIX TESLA SUPERCONDUCTING MAGNET FOR HEAVY-ION BEAM DEFLECTION AND ANALYSIS

R. P. Smith,* L. Bollinger, J. Erskine, and L. Genens*

Bul1. Am. Phys. Soc. 26, 156 (February 1981)

Metal Bonding and Interactions in High Temperature Systems with Emphasis on Alkaline Metals (Proceedings of the Symposium on High Temperature Chemistry, Atlanta, Georgia, 29 March-3 April 1981), ed. by J. L. Gole and W. C. Stwalley (American Chemical Society, 1982)

24. PHOTOIONIZATION AND PHOTOELECTRON SPECTROSCOPY OF ALKALI HALIDE MONOMERS, DIMERS, AND TRIMERS

J. Berkowitz, C. H. Batson, and G. L. Goodman pp. 275-299

Atomic Physics Program Contractors' Workshop, Argonne National Laboratory, Argonne, Illinois, 1-2 April 1981

25. PHOTOIONIZATION AND PHOTOELECTRON SPECTROSCOPY

J. Berkowitz, C. H. Batson, R. Kutina, and G. L. Goodman Progiam, organized by D. S. Gemmell and E. P. Kanter (Argonne National Laboratory, 1981)

26. BEAM-FOIL SPECTROSCOPY AT ARGONNE NATIONAL LABORATORY

H. G. Berry

Program

27. ANALYSIS OF THE BEUTLER-FANO AUTOIONIZATION RESONANCES FOR XeLIKE IONS USING THE RELATIVISTIC MULTICHANNEL QUANTUM DEFECT THEORY

K. T. Cheng

Program

28. LASER-rf DOUBLE-RESONANCE SPECTROSCOPY OF ATOMIC AND MOLECULAR BEAMS

W. J. Childs, David R. Cok, and L. S. Goodman Program

*Accelerator Research Facilities Division, ANL. 
Atomic Physics Program Contractors' Workshop, ANL, April 1981 (cont'd.)

29. THE INTERACTIONS OF FAST MOLECULAR-ION BEAMS WITH MATTER

D. S. Gemmel1, E. P. Kanter, I. Plesser, and M. F. Steuer Program

30. INTERACTION OF ENERGETIC PARTICLES WITH SOLIDS

M. Kaminsky

Program

31. MÖSSBAUER EFFECT RESEARCH

G. J. Perlow

Program

International Conference on Metallurgical Coatings, San Francisco,

California, 6-10 April 1981 (American Vacuum Society, 1981)

32. SPUTTERING OF TiC COATINGS UNDER $\mathrm{D}^{+}$AND ${ }^{4} \mathrm{He}^{+}$ION BOMBARDMENT: TOTAL SPUTTER YIELDS

M. Kaminsky and R. Nielsen

Abstract Booklet, p. 82

33. SURFACE EROSION OF TITANIUM CARBIDE COATINGS UNDER DEUTERIUM AND HELIUM ION IRRADIATIONS

A. S. Rao and M. Kaminsky

Abstract Booklet, p. 82

Proceedings of the Third International Conference on Electrostatic Accelerator Technology, Oak Ridge, Tennessee, 13-16 April 1981, ed. by J. A. Martin (IEEE Service Center, 445 Hoes Lane, Piscataway, New Jersey, 1981), No. 81CH1639-4

34. COMPARATIVE PERFORMANCE OF STRIPPER FOILS IN THE ARGONNE FN TANDEM

P. Den Hartog, G. Thomas, W. Henning, R. Pardo, J. Yntema,

P. Maier-Komor, * and D. Tolfree ${ }^{\dagger}$ pp. $168-171$

35. NEGATIVE ION BEAMS OF RARE AND RADIOACTIVE ISOTOPES

W. Kutschera

pp. 222-227

American Physical Society, Baltimore, Maryland, 20-23 April 1981

36. COMPARISONS BETWEEN THEORY AND EXPERIMENT IN TWO- AND THREEELECTRON SYSTEMS

H. G. Berry

Bul1. Am. Phys. Soc. 26, 629 (April 1981)

\footnotetext{
* Technical Iniversity of Munich, Garching, West Germany.

${ }^{+}$Nuclear Structure Facility, Daresbury Laboratory, U.K.
} 
APS, Raltimore, April 1981 (cont'd.)

37. MASS AND CHARGE DISTRIBUTIONS IN THE $270 \mathrm{MeV}{ }^{37} \mathrm{Cl}$ on ${ }^{40} \mathrm{Ca}$ AND $209_{\text {Bi REACTIONS }}$

A. C. Mignerey, * H. Breuer, * K. L. Wolf, ${ }^{\dagger}$ B. G. Glagola, ${ }^{\dagger}$

C. Davids, R. R. Betts, ${ }^{\dagger}$ and V. E. Viola $\neq$

Bull. Am. Phys. Soc. 26, 539 (April 1981)

38. HIGH-SPIN YRAST STATES IN ${ }^{154}$ Dy

A. Pakkanen, 5 Y. H. Chung, 5 P. J. Daly, 5 S. R. Faber, 5 H.

Helppi, $\S$ J. Wilson, $\xi$ I. Ahmad, $\dagger$ J. Borggreen, P. Chowdhury,

Z. Grabowski, T. L. Khoo, and R. K. Smither

Bul1. Am. Phys. Soc. 26, 620 (April 1981)

39. SYSTEMATICS OF THE ${ }^{24} \mathrm{Mg}\left({ }^{16} 0,{ }^{12} \mathrm{C}\right){ }^{28}$ S1 REACTION FOR $23 \mathrm{MeV} \leqslant E_{\mathrm{c}, \mathrm{m}}$. $\leqslant 53 \mathrm{MeV}$

S. J. Sanders, $\|$ H. Ernst, W. Henning, and J. Barrette

Bull. Am. Phys. Soc. 26, 553 (April 1981)

40. MEASUREMENT OF THE RECOIL DEUTERON TENSOR POLARIZATION IN $\pi-d$ SCATTERING AT $\mathrm{T}_{\pi}=142 \mathrm{MeV}$

K. Stephenson, R. J. Holt, J. R. Specht, B. Zeidman, J. S, Frank, ** M. J. Leitch,** J. D. Moses, ** E. J. Stephenson, $\neq$ and R. M. Laszewskitt

Bull. Am. Phys. Soc. 26, 625 (April 1981)

41. YRAST SPECTROSCOPY OF THE $\mathrm{N}=83$ NUCLEUS ${ }^{150}$ HO

J. Wilson, $\S$ Y. H. Chung, $\S$ S. R. Faber, $\S$ A. Pakkanen, $\$$ P. J.

Daly, $\$$ I. Ahmad, $†$ P. Chowdhury, T. L. Khoo, R. D. Lawson, and

R. K. Smither

Bull. Am. Phys. Soc. 26, 620 (April 1981)

Symposium on Accelerator Mass Spectrometry, Argonne National Laboratory, Argonne, Illinois, 11-13 May 1981

42. ACCELERATOR MASS SPECTROMETRY OF ${ }^{59} \mathrm{Ni}$ AND Fe ISOTOPES AT THE ARGONNE SUPERCONDUCTING LINAC

W. Henning, W. Kutschera, B. Myslek-Laurikainen, R. C. Pardo,

R. K. Smither, and J. L. Yntema

Proceedings of the Symposium, organized by $W$. Henning, W. Kutschera, R. K. Smither, and J. L. Yntema, Physics Division Informal Report ANL-PHY-81-1 (May 1981), Pp. 320-329

+ Program of the Symposium (ANL, 1981)

\footnotetext{
*University of Maryland, College Park, Maryland.

${ }^{\dagger}$ Chemistry Division, ANL.

‡Indiana University, Bloomington, Indiana.

${ }^{5}$ Purdue University, West Lafayette, Indiana.

Yale University, New Haven, Connecticut.

"Brookhaven National Laboratory, Upton, New York.

** Los Alamos National Laboratory, Los Alamos, New Mexico.

${ }^{+t}$ University of Illinois, Urbana, I11inois.
} 
Accelerator Mass Spectrometry, ANL, May 1981 (cont'd.)

43. THE ROLE OF ACCELERATOR MASS SPECTROMETRY IN NUCLEAR PHYSICS

W. Kutschera

Proceedings of the Symposium, Pp. 43-56

+ Program of the Symposium

44. RECENT SEARCH FOR QUARKS AND VERY HEAVY HYDROGEN ISOTOPES USING AN (ALMOST) ALL-ELECTROSTATIC SYSTEM

J. P. Schiffer, H. Ernst, W. Henning, and W. Kutschera

Proceedings of the Symposium, pp. 154-156

+ Program of the Symposium

Proceedings of the 5th High Energy Heavy Ion Study, Berkeley, California, 18-22 May 1981, ed. by G. S. Pappas (Lawrence Berkeley Laboratory, Berkeley, California, 1981), Lawrence Berkeley Laboratory Report LBL-12652

45. INTERACTION AND COLLECTIVE EFFECTS IN CLASSICAL-EQUATIONS-OFMOTION CALCULATIONS

A. R. Bodmer

pp. $648-654$

Dynamics of Heavy-Ion Collisions (Proceedings of Europhysics Conference, Hvar, Yugoslavia, 25-31 May 1981), ed. by N. Cindro, R. A. Ricci, and W. Greiner (North-Holland, Amsterdam, 1981)

46. RESONANCES IN HEAVY-ION COLLISIONS: EXPERIMENTAL STATUS W. Henning

pp. $75-99$

4th International Conference on Nuclei Far from Stability, Helsing Denmark, 7-13 June 1981, CERN 81-09 (CERN, Geneva, 1981)

47. ON-LINE LASER SPECTROSCOPY AT THE ARGONNE SUPERCONDUCTING LINAC C. N. Davids, G. W. Greenlees,* M. A. Finn,* D. A. Lewis, ${ }^{\dagger}$ and R. M. Evanst pp. 42-43

36th Annual Congress of the Canadian Association of Physicists, Halifax, Nova Scotia, 15-18 June 1981

48. ENHANCEMENT OF LYMAN SERIES EMISSION FOLLOWING BEAM-FOIL DISSOCIATION OF $\mathrm{H}_{2}{ }^{+}$AND $\mathrm{H}_{3}{ }^{+}$

R. L. Brooks, H. G. Berry, and J. Hardis

Bull. Canadian Assn. Physicists $37(3), 7$ (1981)

Laser Spectroscopy V (Proceedings of the Fifth International Conference, Jasper, Alberta, Canada, 29 June-3 July 1981), ed. by A. R. W. McKellar, T. Oka, and B. P. Stoicheff (Springer-Verlag, Berlin-Heidelberg, 1981)

49. MOLECULAR-BEAM, LASER-RF, DOUBLE RESONANCE STUDIES OF CALCIUM MONOHALIDE RADICALS

W. J. Childs, D. R. Cok, and L. S. Goodman

pp. 150-153

\footnotetext{
*University of Minnesota, Minneapolis, Minnesota.

† Iowa State University, Ames, Iowa.
} 
Neutrino 81 (Proceedings of the 1981 International Conference on Neutrino Physics and Astrophysics, Maui, Hawaii, 1-8 July 1981), ed. by R. J. Cence, E. Ma, and A. Roherts (University of Hawaii, Honolulu, 1981.), Vol. I

50. UNCERTAINIIES IN THE CALCULATION OF SOLAR NEUTRINO CAPTURE RATES

B. W. Filippone

pp. $19-26$

Ninth International Conference on Atomic Collisions in Solids, Lyon, France, 6-10 July 1981

51. COLLISIONAL EFFECTS IN THE PASSAGE OF FAST MOLECULAR IONS THROUGH THIN FOILS

Donald S. Gemmell

Nucl. Instrum. Methods 194, 255-267 (15 March 1982)

+ Program of the Conference, Abstracts, p. 36

52. ION-SOURCE DEPENDENCE OF THE DISTRIBUTIONS OF INTERNUCLEAR SEPARATIONS IN $2 \mathrm{MeV} \mathrm{HeH}{ }^{+}$BEAMS

Elliot P. Kanter, Donald S. Gemmell, Itzhak Plesser, and Zeev Vager

Nucl. Instrum. Methods $194,307-310$ (15 March 1982)

+ Program of the Conference, Abstracts, p. 57

53. DIMINISHED STOPPING POWER FOR FAST NITROGEN AND OXYGEN DICLUSTERS IN CARBON

Malcolm F. Steuer, Donald S. Gemmell, Elliot P. Kanter,

Edward A. Johnson, and Bruce J. Zabransky Nucl. Instrum. Methods 194, 277-279 (15 March 1982)

+ Program of the Conference, Abstracts, p. 21

9 ICOHEPANS ( 9 th International Conference on High Energy Physics and Nuclear Structure, Versailles, France, 6-10 July 1981) (CEN, Saclay, 1981)

54. INCLUSIVE $\pi^{+}$SCATTERING FROM ${ }^{4} \mathrm{He}$

D. F. Geesaman, S. M. Levenson, E. P. Colton,* R. J. Holt, H. E. Jackson, J. P. Schiffer, J. R. Specht, K. E. Stephenson, B. Zeidman, R. E. Segel, $\dagger$ P. A. M. Gram, $\neq$ and C. A. Goulding $\xi$ Abstracts of Contributed Papers, p. 344

$*$

*Accelerator Research Facilities Division, ANL.

Torthwestern University, Evanston, Illinois.

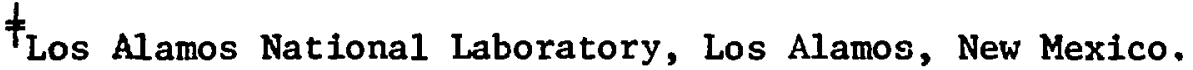

Florida A \& M University, Tallahassee, Florida. 
High Energy Physics and Nuclear Structure, Versallles, July 1981 (cont'd.)

55. INELASTIC PION SCATTERING FROM ${ }^{10} 0_{B}$ AND ${ }^{11_{B}}$

D. F. Geesaman, B. Zeidman, P. Zupranski,* R. E. Segel,*

C. L. Morris, ${ }^{\dagger}$ R. L. Boudrie, ${ }^{\dagger}$ S. Greene, $\neq$ B. G. Ritchie,, 5

G. S. Blanpied, $\$$ C. L. Harvey, $\|$ L. W. Swenson, $\pi$ and G. C. Morrison**

Abstracts of Contributed Papers, p. 302

Microbeam Analysis - 1981 (Proceedings of the 16th Annual Conference of the Microbeam Analysis Society, Vail, Colorado, 13-17 July 1981), edited by Roy H. Geiss (San Francisco Press, San Francisco, 1981)

56. AN ALTERNATIVE APPROACH TO THE SURFACE-EXCITATION MODEL IN SIMS

V. E. Krohn

pp. 296-298

XII Internat: onal Confersnce on the Physics of Electronic and Atomic

Collisions, Catlinburg, Ter.iessee, 15-21 July 1981

57. MOLECULAR-ION STRUC'TURES DETERMINED FROM COULOMB EXPLOSIONS OF PENETRATING IONS

Donald S. Gemmell

Physics of Electronic and Atomic Collisions (Proceedings of the Conference), ed. by S. Datz (North-Holland, Amsterdam, 1982), pp, 841-860

58. PHOTOIONIZATION AND PHOTOELECTRON SPECTROSCOPY OF OPEN-SHELL ATOMS: ATOMIC TELLURIUM AND ATOMIC IODINE

G. L. Goodman and J. Berkowitz

Abstracts of Contributed Papers, Vo1. 2, pp. 1169-1170

59. PHOTOIONIZATION MASS SPECTROMETRY OF $\mathrm{NH}_{2} \mathrm{OH}$ : THE ENTHALPIES OF FORMATION OF HNO ${ }^{+}$AND $\mathrm{NOH}^{+}$

R. J. Kutina and J. Berkowitz Abstracts of Contributed Papers, vol. 2, pp. 1171-1172

60. PHOTOIONIZATION STUDIES OF 5d AND 6s SHELLS OF ATOMIC MERCURY

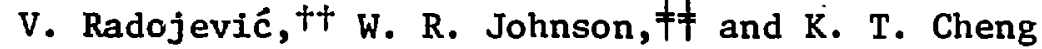
Abstracts of Contributed Papers, Vo1. 1, pp. 28-29

*Northwestern University, Evanston, Illinols.

tLos Alamos National Laboratory, Los Alamos, New Mexico.

$\#_{\text {New Mexico State University, Las Cruces, New Mexico. }}$

${ }^{\S}$ University of South Carolina, Columbia, South Carolina.

University of Texas, Austin, Texas.

"Oregon State University, Corvallis, Oregon.

** University of Birmingham, Birmingham, England.

${ }^{+\dagger}$ Boris Kidric Institute, Beograd, Yugaslavia.

\# Notre Dame UnIversity, Notre Dame, Indiana. 
Second Topical Meeting on Fusion Reactor Materials, Seattle, Washington, 9-12 August 1981

61. THE EFFECTS OF LIGHT, GAS ION BOMBARDMENT ON THE STRUCTURE OF GRAPHITE AND SILICON CARBIDE

D. J. Bacon, * I. Dumler, * and A. S. Rao Program of the Meeting (American Nuclear Society, 1981), pp. $5 \mathrm{~B}-14-5 \mathrm{~B}-14 \mathrm{a}$

62. THE EFFECT OF DOSE ON THE EVOLUTION OF CAVITIES ON $20-\mathrm{keV}^{4} \mathrm{He}^{+}$ ION IRRADIATED NICKEL

G. Fenske, ${ }^{\dagger}$ S. K. Das, and M. Kaminsky

Program of the Meeting, pp. 4G-8-4G-8a

63. SURFACE ROUGHNESS FACTOR MEASUREMENTS OF STAINLESS STEELS WITH ENERGETIC ION IRRADIATION AND DISCHARGE CLEANING

S. Maeda, $\neq$ M. Mohri, $\neq$ T. Yamashina, $\neq$ A. G. Mathewson, $\S$ and

M. Karninsky

Program of the Meeting, pp. 2E-7-2E-7a

64. SUPPRESSION OF REPETITIVE SURFACE EXFOLIATION OF INCONEL 625 IMPLANTED SEQUENTIALLY WITH HELIUM IONS OF DIFFERENT ENERGIES $(20-100 \mathrm{keV})$

A. S. Rao, M. Kaminsky, and J. L. Whitton

Program of the Meeting, pp. 3B-12-3B-12a

Workshop on High-Resolution, Large-Acceptance Spectrometers, Argonne National Laboratory, Argonne, Illinois, 8-11 September 1981, ed. by B. Zeidman, Physics Division Informal Report AN. $\mathrm{AHY}-81-2$

65. A DESIGN FOR THE LARGE ELECTRON SPECTROMETER (LES)

B. Zeidman

pp. V.J-1-J-9

$\mathrm{X}$-Ray Astronomy in the 1980's (Proceedings of a Workshop on X-Ray Astronomy and Spectroscopy, Greenbelt, Maryland, 5-7 October 1981), ed. by S. S. Holt (Goddard Space Flight Center, Greenbelt, Maryland, 1981), NASA Technical Memorandum 83848

66. A NEW METHOD FOR FOCUSING AND IMAGING X RAYS AND GAMMA RAYS WITH DIFFRACTION CRYSTALS

Robert K. Smither

pp. 477-495

The University, Liverpool, England.

$\dagger_{\text {Materials Science Division, ANL. }}$

† Hokkaido University, Sapporo, Japan.

${ }^{5}$ CERN, Geneva, Switzerland. 
S.N.E.A.P. 1981 (Proceedings of the Symposium of Northeastern Accelerator Personnel, Niagara-on-the-Lake, Ontario, Canada, 14-16 October 1981), ed. by J. W. McKay (McMaster University, Hamilton, Ontario, Canada, 1981)

67. ARGONNE NATIONAL LABORATORY 1980-81 TANDEM-LINAC ACCELERATOR REPORT

P. Den Hartog, R. Pardo, F. Munson, and C. Heath pp. $76-82$

Proceedings of the 1981 Linear Accelerator Conference, Santa Fe, New Mexico, 19-23 October 1981, ed. by R. A. Jameson and L. S. Taylor, Los Alamos National Laboratory Report LA-9234-C (February 1982)

68. STATUS OF THE ARGONNE SUPERCONDUCTING-LINAC HEAVY-ION BOOSTER J. Aron,* R. Benaroya, L. M. Bollinger, B. E. Clifft,* K. W. Johnson,* P. Markovich,* J. M. Nixon,* R. Pardo, K. W. Shepard, and G. Zinkann pp. $25-28$

69. LONGITUDINAL BEAM OPTIMIZATION OF THE ARGONNE SUPERCONDUCTING HEAVY-ION LINEAR ACCELERATOR

R. Pardo, L. M. Bollinger, and W. Henning pp. $270-273$

Division of Nuclear Physics, American Physical Society, Asilomar, California, 28-30 October 1981

70. $\triangle$ RESONANCES IN NUCLEI

D. Ashery Bu11. Am. Phys. Soc. 26, 1130 (October 1981)

71. THE ISOSPIN DEPENDENCE OF PION ABSORPTION ON A PAIR OF NUCLEONS

D. Ashery, R. J. Holt, H. E. Jackson, J. P. Schiffer, J. R. Specht, K. E. Stephenson, R. D. Mckeown, $t$ J. Ungar, ${ }^{+}$R. E. Segel, $\neq$ and P. Zupranskif

Bul1. Am. Phys. Soc. 26, 1125 (October 1981)

72. $\left(\pi \mathrm{h}_{11 / 2}\right)^{4}$ SPECTRUM OF THE N=82 NUCLEUS ${ }^{150} \mathrm{Er}$

Y. H. Chung, 5 P. J. Daly, 5 S. R. Faber, 5 Z. W. Grabowski, 5

H. Helppi, $\S$ A. Pakkanen, $\$$ J. Wilson, $\$$ I. Ahmad, * P. Chowdhury, T. L. Khoo, and R. D. Lawson Bull. Ail. Phys. Soc. 26, 1155 (October 1981)

73. MEASUREMENT OF COMPOUND NUCLEAR $\mathrm{K} \times$ X-RAY PRODUCTION CROSS SECTIONS FOR $32 \mathrm{~S}+116,120,124 \mathrm{Sn}$

H. Ernst, C. N. Davids, W. Freeman, W. Henning, and T. Humanic Bull. Am. Phys. Soc. 26, 1133 (October 1981)

*Chemistry Division, ANL.

$+$

California Institute of Technology, Pasadena, California.

$\ddagger_{\text {Northwestern University, Evanston, Illinois. }}$

${ }^{5}$ Purdue University, West Lafayette, Indiana. 
APS, Asilomar, October 1981 (cont'd.)

74. THE FIVE VALENCE PROTON $\mathrm{N}=82$ NUCLEUS ${ }^{151} \mathrm{Tm}$

H. Helppi,* Y. H. Chung, * P. J. Daly,* Z. W. Grabowski, *

A. Pakkanen, * I. Ahmad, $\dagger$ P. Chowdhury, T. L. Khoo, and R. D. Lawson

Bu11. Am. Phys. Soc. 26, 1155 (October 1981)

75. INELASTIC SCATTERING, TRANSFER REACTIONS, AND Z-DISTRIBUTION MEASUREMENTS FOR ${ }^{160}+{ }^{48} \mathrm{Ca}$ AT $E_{1 \mathrm{ab}}=158 \mathrm{MeV}$

T. J. Humanic, H. Ernst, W. Henning, and B. Zeidman Bull. Am. Phys. Soc. 26, 1152 (October 1981)

76. GAMMA-RAY SPECTROSCOPY OF ${ }^{180} \mathrm{Ta}$

E. B. Norman, S. E. Kellogg, $\neq$ and T. R. Renner Bul1. Am. Phys. Soc. 26, 1116 (October 1981)

77. TRANSITION FROM COLLECTIVE TO SINGLE-PARTICLE NATURE AT HIGH SPIN IN 154 Dy

A. Pakkanen, * Y. H. Chung, * P. J. Daly, * S. R. Faber, * H.

Helppi,* J. Wilson,* T. L. Khoo, P. Chowdhury, I. Ahmad, ${ }^{\dagger}$

Z. Grabowski, and D. C. Radford $\$$

Bul1. Am. Phys. Soc. 26, 1116 (October 1981)

78. FUSION OF ${ }^{16} \mathrm{O}+{ }^{24} \mathrm{Mg}$

F. W. Prosser, Jr., $\|$ R. A. Racca,\| K. Daneshvar, C. N. Davids, and D. G. Kovar

Bu11. Am. Phys. Soc. 26, 1132 (October 1981)

79. FUSION OF ${ }^{18} 0+{ }^{24} \mathrm{Mg}$ AND ${ }^{16} 0+{ }^{26} \mathrm{Mg}$

R. A. Racca, $\|$ F. W. Prosser, Jr.,\| K. Daneshvar, C. N. Davids, and D. G. Kovar

Bul1. Am. Phys. Soc. 26, 1132-1133 (October 1981)

80. THE NUMBER OF NUCLEONS INVOLVED IN PION ABSORPTION AT $500 \mathrm{MeV}$ K. E. Stephenson, D. Ashery, R. J. Holt, H. E. Jackson, J. P. Schiffer, J. R. Specht, R. D. Mckeown, $\pi$ J. Ungar, $\pi$ R. E. Segel, ** and $P$. Zupranski**

Bul1. Am. Phys. Soc. 26, 1125 (October 1981)

\footnotetext{
* Purdue UnIversity, West Lafayette, Indiana.

†Chemistry Division, ANL.

\#University of Washington, Seattle, Washington.

${ }^{5}$ Yale University, New Haven, Connecticut.

University of Kansas, Lawrence, Kansas.

"California Institute of Technology, Pasadena, California.

** Northwestern University, Evanston, Illinois.
} 
28th National Symposium of the American Vacuum Soclety, Anaheim, California, 2-6 November 1981

81. PREFERENTIAL SPUTTERING OF TIC AND TiB 2 COATINGS UNDER $\mathrm{D}^{+}$AND ${ }^{4} \mathrm{He}^{+}$ BOMBARDMENT: PARTIAL YIELDS

M. Kaminsky, R. Nielsen, and P. Zschack Program, p. 127

Division of Electron \& Atomic Physics, American Physical Society, New York, New York, 3-5 December 1981

82. MULTICHANNEL QUANTUM DEFECT ANALYSIS OF THE ABSORPTION SPECTRUM OF $\mathrm{Ba}^{2+}$

K. T. Cheng and W. R. Johnson*

Bul1. Am. Phys. Soc. 26, 1325 (November 1981)

83. THE COULOMB EXPLOSION OF CHANNELED HeH ${ }^{+}$BEAMS

E. P. Kanter, D. S. Gemmell, M. F. Steuer, and B. J. Zabransky Bul1. Am. Phys. Soc. 26, 1309 (November 1981)

84. ELECTRON EMISSION FOLLOWING FAST MOLECULAR ION IMPACT ON GAS AND FOIL TARGETS

D. Schneider, B. J. Zabransky, E. P. Kanter, D. S. Gemme11, and $\mathrm{E}$. Johnson

Bul1. Am. Phys. Soc. 26, 1309 (November 1981)

\section{PHYSICS DIVISION REPORTS}

1. PROCEEDINGS OF THE SYMPOSIUM ON ACCELERATOR MASS SPECTROMETRY, Argonne National Laboratory, Argonne, Illinois, 11-13 May 1981 organized by W. Henning, W. Kutschera, R. K. Smither, and J. L. Yntema

Physics Division Informal Report ANL-PHY-81-1 (May 1981)

2. WORKSHOP ON HIGH-RESOLUTION, LARGE-ACCEPTANCE SPECTROMETERS, Argonne National Laboratory, Argonne, Illinois, 8-11 September 1981 edited by B. Zeidman

Physics Division Informal Report ANL-PHY-81-2

*University of Notre Dame, Notre Dame, Indiana. 


\section{STAFF MEMBERS OF THE PHYSICS DIVISION}

Listed below are the permanent staff of the Physics Division for the year ending 31 March 1982. The program heading indicates only the individual's current primary activity.

\section{EXPERIMENTAL NUCLEAR PHYSICS}

\section{Scientific Staff}

* Lowell M. Bollinger, Ph.D., Cornell University, 1951

Cary N. Davids, Ph.D., California Institute of Technology, 1967

+ A

Alexander J. Elwyn, Ph.D., Washington University, 1956

F Melvin S. Freedman, Ph.D., University of Chicago, 1942

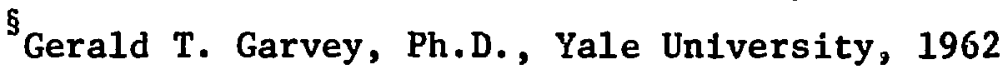

Donald F. Geesaman, Ph.D., State Univers1ty of New York, Stony Brook, 1976

Walter Henning, Ph.D., Technical University, Munich, 1968

/Robert E. Holland, Ph.D., University of Iowa, 1950

Roy J. Holt, Ph.D., Yale University, 1972

IHarold E. Jackson, Jr., Ph.D., Cornell University, 1959

Robert V. Janssens, Ph.D., Université Catholique de Louvain, Belgium, 1978

Teng Lek Khoo, Ph.D., McMaster University, 1972

*

Dennis G. Kovar, Ph.D., Yale University, 1971

Victor E. Krohn, Ph.D., Case Western Reserve University, 1952

Walter Kutschera, Ph.D., University of Graz, Austria, 1965

${ }^{+\dagger}$ Alexander Langsdorf, Jr., Ph.D., Massachusetts Institute of Technology, 1937

Richard C. Pardo, Ph.D., University of Texas, 1976

* In charge of tandem-linac operations and the ATLAS project.

${ }^{\dagger}$ In charge of Dynamitron accelerator operations.

$\ddagger_{\text {Retired - research participant. }}$

§oint appointment with the University of Chicago.

$\|_{\text {Retired - consultant as of March } 1982 .}$

"In charge of Design Project for GeV Electron Microtron.

** Temporarily assigned to Hahn-Meitner-Institüt für Kernforschung, Berlin, Germany (September 1980-August 1981).

${ }^{+}{ }^{+}$Retired - consultant. 
Karl Ernst Rehm, Ph.D., Technical University, Munich, 1973

${ }^{*}$ G. Roy Ringo, Ph.D., University of Chicago, 1940

†John P. Schiffer, Ph.D., Yale University, 1954

Kenneth W. Shepard, Ph.D., Stanford University, 1970

\# Harunori Takeda, Ph.D., University of Pennsylvania, 1978

${ }^{5}$ J. L. Yntema, Ph.D., Free University of Amsterdam, 1952

Benjamin Zeidman, Ph.D., Washington University, 1957

Technical and Engineering Staff

Ralph Benaroya

$\|_{\text {John J. Bicek }}$

Peter J. Billquist

John M. Bogaty

"Patric K. Den Hartog

William F. Evans

** Walter A. Huston

Joseph E. Kulaga

Bruce G. Nardi

Robert W. Nielsen

James R. Specht

t+

James L. Stadelmann

George E. Thomas, Jr.

James $\mathrm{N}$. Worthington

Jerome R. Wrobel

Gary P. Zinkann

* Retired - consultant as of March 1982.

tDirector of the Physics Division. Joint appointment with the University of Chicago.

$干_{\text {No }}$ longer at Argonne as of January 1982. Present address: K.M.S. Fusion Inc., Ann Arbor, Michigan.

§ In charge of Tandem accelerator operations through May 1981.

Wo longer at Argonne as of September 1981.

In charge of Tandem accelerator operations as of June 1981.

** No longer at Argonne as of March 1982.

${ }^{+}{ }_{\text {No }}$ longer at Argonne as of October 1981. 
THEORETICAL NUCLEAR PHYSICS

\section{Scientific Staff}

* Arnold R. Bodmer, Ph.D., Manchester University, 1953

Fritz Coester, Ph.D., University of Zurich, 1944

Benjamin Day, Ph.D., Cornell University, 1963

Dieter Kurath, Ph.D., University of Chicago, 1951

Robert D. Lawson, Ph.D., Stanford University, 1953

Tsung-Shung Harry Lee, Ph.D., University of Pittsburgh, 1973

James E. Monahan, Ph.D., St. Louis University, 1953

† Murray Peshkin, Ph.D., Cornell University, 1951

Steven C. Pieper, Ph.D., University of Illinols, 1970

ATOMIC AND MOLECULAR PHYSICS

\section{Scientific Staff}

Joseph Berkowitz, Ph.D., Harvard University, 1955

$\ddagger_{H}$. Gordon Berry, Ph.D., University of Wisconsin, 1967

Kwok-tsang Cheng, Ph.D., University of Notre Dame, 1977

William J. Childs, Ph.D., University of Michigan, 1956

${ }^{5}$ Donald S. Gemme1l, Ph.D., Australian National University, 1960

Leonard S. Goodman, Ph.D., University of Chicago, 1952

Elliot P. Kanter, Ph.D., Rutgers University, 1977

II Gilbert J. Perlow, Ph.D., University of Chicago, 1940

"Dieter Schnelder, Ph.D., Free University of Berlin, 1975

*Joint appointment with the University of Illinois, Chicago Circle Campus.

${ }^{\dagger}$ Deputy Director of the Physics Division.

$\ddagger_{\text {Temporarily assigned to Laboratolre Alme Cotton, C.N.R.S., Orsay, France }}$ (November 1981-April 1982).

5 Associate Director of the Physics Division.

$\|_{\text {Joint appointment as Editor of the Applied Physics Letters. }}$

"Formerly Resident Associate; joined the permanent staff 1 February 1982. 


\section{Technical and Engineering Staff}

$*$

Walter J. Ray

Bruce J. Zabransky

\section{SURFACE SCIENCE-FUSION POWER}

\section{Scientific Staff}

Manfred S. Kaminsky, Ph.D., University of Marburg, 1957

\section{ADMINISTRATIVE STAFF}

${ }^{\dagger}$ Albert J. Hatch, M.S., University of Illinois, 1947

${ }^{F_{F}}$. Paul Mooring, Ph.D., University of Wisconsin, 1951

* No longer at Argonne as of September 1981.

${ }^{\dagger}$ Assistant Director of the Physics Division. No longer at Argonne as of September 1981.

FAssistant Director of the Physics Division. 
From 1 April 1981 through 31 March 1982 there were 38 temporary staff members and visitors (including 13 postdoctoral appoiritees), 9 graduate students, and 7 undergraduates. These temporary appointments in the Physics Division are listed below.

TEMPORARY APPOINTMENTS

\section{Postdoctoral Appointees}

Robert L. Brooks (from University of Alberta, Alberta, Canada): Study of fast ion-beam interactions with matter; spectroscopic analysis of atomic and molecular interaction parameters. (August 1979-October 1981)

Partha Chowdhury (from State University of New York, Stony Brook, New York): Studies of high-spin states of nuclei. (November 1979- )

David R. Cok (from Harvard University, Cambridge, Massachusetts): RF laser spectroscopy. (September 1980- )

Hartmut J. H. Ernst (Technical University of Munich, Munich, Germany): Experimental heavy-ion and charged-particle nuclear physics. (FeodorLynen Fellowship of the Alexander v. Humboldt Foundation.) (January 1980-March 1982)

Wiliiam S. Freeman (from State University of New York, Stony Brook, New York): Experimental heavy-ion and charged-particle nuclear physics. (December 1980- )

Thomas J. Humanic (from University of Pittsburgh, Pittsburgh, Pennsylvania): Experimental heavy-ion and charged-particle nuclear physics. (August 1980- )

Wolfgang Kühn (from Max-Planck-Institut für Kernphysik, Heidelberg, Germany): Study of nuclear reactions with the linac and intermediate energy physics at LAMPF. (May 1981- )

Raymond E. Kutina (from University of Toronto, Toronto, Canada): Photoionization of free radicals. (March 1980- )

*

Samuel M. Levenson (from University of Chicago, Chicago, Illinois): Nuclear physics research. (January 1979-March 1982)

John A. Parmentola (from Massachusetts Institute of Technology, Cambridge, Massachusetts): Quark bag models; multiple scattering theory. (August 1980- )

Branko M. Ruscic (from Rudjer Boskovic Institute, Zagreb, Yugoslavia): Photolonization and photoelectron spectroscopy. (September i981- )

Kenneth E. Stephenson (from University of Wisconsin, Madison, Wisconsin): Pion-nucleus reaction experiments. (September 1979-)

\footnotetext{
${ }^{\star}$ Formerly DEP Lab-Grad Program Thesis Student, through December 1981.
} 
Robert B. Wiringa (from Los Alamos National Laboratory, Los Alamos, New Mexico): NN interaction, three-body forces, many-body theory for light nuclei, nuclear matter and neutron stars. (January 1981-) )

\section{Long-Term Visitors (at Argonne more than 4 months)}

Ademola Amusa (University of Ife, Ile-Ife, Nigeria, Africa): Shell-model calculations in some nuclei (e.g., 200). (May 1980-) )

Daniel Ashery (Tel Aviv University, Ramat Aviv; Israel): Pion-nucleus reactions. (August 1980- )

*

Thomas W. Dombeck (University of Maryland, College Park, Maryland): Measurement of the neutron electric dipole moment. (October 1980July 1981)

Steven R. Faber (Purdue University, West Lafayette, Indiana): High-spin physics by gamma-ray spectroscopy. (September 1979-August 1981)

Stuart J, Freedman (Stanford University, Stanford, California): Weak interactions research. (Alfred P. Sloan Fellow.) (January 1981-

Dieter Frekers (University of Mïnster, Münster, West Germany): Experimental heavy-ion and charged-particle nuclear physics.

(December 1981- )

Zbigniew W. Grabowski (Purdue University, West Lafayette, Indiana): Heavy-ion nuclear physics. (September 1980-August 1981)

Mitsuji Kawai (Kyushu University, Fukuoka, Japan): Theory of nuclear reactions, especially theory and application of the method of coupled channels to multistep nuclear rearrangement processes. (October 1980April 1981)

†Harry J. Lipkin (Weizmann Institute of Science, Rehovot, Israel): Implications of quark model for hadrons; grand unification groups; and independent boson model for nuclei. (June 1979-October 1981)

Malcolm F. Steuer (University of Georgia, Athens, Georgia): Studies of stopping power of carbon for $1-3-\mathrm{MeV} \mathrm{N}_{2}{ }^{+}$ions. (August 1980August 1981)

\section{Resident Graduate Students}

Philip W. Arcuni (University of Chicago, Chicago, Illinois): Electron spectroscopy of ion-atom collisions. (October 1981-)

* Joint appointment with Los Alamos National Laboratory, Los Alamos, New Mexico.

tJolnt appolntment with the Fermi National Accelerator Laboratory, Batavia, Illinois. 
Alexandra R. Davis (University of Chicago, Chicago, Illinois): Weak interactions in nuclear physics. (January 1979- )

Rollin Evans (Iowa State University, Ames, Iowa): On-1ine laser spectroscopy of radioactive atoms using the superconducting linac. (March 1981- )

Bradley W. Filippone (University of Chicago, Chicagc, Illinois): Experimental nuclear astrophysics. (June 1979- )

Miles A. Finn (University of Minnesota, Minneapolis, Minnesota): On-1ine laser spectroscopy of radioactive atoms using the superconducting linac. (April 1981- )

Carl A. Gagliard1 (Princeton University, Princeton, New Jersey): Weak interactions in nuclear physics. (July 1977- )

Jonathan E. Hardis (University of Chicago, Chicago, Illlinois): Ion-ion and ion-atom collisions. (October 1979- )

Short-Term Visitors (at Argonne less than 4 months)

\section{A. Faculty}

Larry E. Campbell (Hobart \& William Smith Colleges, Geneva, New York): Photovoltaic solar cell research. (June 1981-August 1981)

Alan K. Edwards (University of Georgia, Athens, Georgia): UV-laser photofragmentation of molecular ions. (June 1981-August 1981)

Adoram Erell (University of Tel-Aviv, Tel-Aviv, Israe1): ANL experiment \#303 at Los Alamos National Laboratory. (October 1981-November 1981)

Yuan zhuang Gu (Fudan University, Shanghai, China): Experiments associated with molecular beams and ion beams while going through thin foils or gas targets. (October 1981-Januacy 1982)

Donald Koetke (Valparaiso University, Valpara1so, Indiana): Cross sections for the $7_{\mathrm{Li}}(\mathrm{d}, \mathrm{p})^{8} \mathrm{Li}$ and $7_{\mathrm{Be}}(\mathrm{p}, \gamma)^{8} \mathrm{~B}$ reactions at low energies. (June 1981-August 1981)

David A. Lewis (Iowa State University, Ames, Iowa): On-Iine laser specEroscopy of radioactive atoms using the superconducting 1 inac.

A. Eugene Livingston (University of Notre Dame, Notre Dame, Indiana): Beam-foil spectroscopy of neutral and ionized atoms.

Malcolm H. Macfarlane (Indiana University, Bloomington, Indiana): Coupledchannels description of heavy-ion inelastic scattering. (July 1981)

KaI Neergard (University of Gilessen, Giessen, West Germany): Interpretation of data on 11ght Dy isotopes, particularly side-feeding patterns and continuum spectra. (nugust 1981-October 1981) 
Michael Paul (Hebrew University, Jerusalem, Israel): Accelerator mass spectrometry; massive transfer nuclear reactions. (August 1981September 1981)

Itzhak Plesser (Welzmann Institute of Science, Rehovot, Israel): Studies of molecular ion beam explosion, while going through thin foils. (May 1981-June 1981)

Ove Poulsen (University of Aarhus, Aarhus, Dennark): Rf-pumped laser spectroscopy of CaCl. (July 1981)

Stephen J. Sanders (Yale University, New Haven, Connecticut): Study of resonance-like structures observed in heavy-ion reactions. (July 1981)

Ralph E. Segel (Northwestern University, Evanston, I1linois): Nuclear physics research. (September 1981)

Zeev Vager (Weizmann Institute of Science, Rehovot, Israel): Interaction of fast molecular ions with solids. (September 1981-October 1981)

\section{B. Graduate Students}

Jordan B. Camp (University of Chicago, Chicago, Illinois): (March 1981May 1981)

Oded Meirav (Hebrew University, Jerusalem, Israel): Heavymion detector development. (August 1981-September 1981)

\section{Undergraduate Students}

Jose L. Balduz, Jr. (Tulane University, New Orleans, Louisiana): (May 1981-August 1981)

Richard W. Chamberl.in, Jr. (Illinois Institute of Technology, Chicago, Illinois).

Richard A. Goldstein (Princeton University, Princeton, New Jersey). (June 1981-August 1981)

James J. Hofmann (East Stroudsburg State College, East Stroudsburg, Pennsylvania). (January 1982- )

Edward A. Johnson (Washington \& Lee University, Lexington, Virginia).

William 0. Perkins (North Carolina State University, Raleigh, North Carolina).

Paul R. Zschack (I1linois Institute of Technology, Chicago, Illinois). 